Supplementary material belonging to the publication:

\title{
"Unexpected" 29Si NMR Chemical Shifts in Heteroatom-Substituted Silyllithium Compounds: A Quantum Chemical Analysis
}

\author{
Dominik Auer, Martin Kaupp* and Carsten Strohmann ${ }^{*}$ \\ Institut für Anorganische Chemie, Universität Würzburg, Am Hubland, Würzburg, Germany
}

Table of contents for supplementary material

pages

$\begin{array}{lc}\text { Selected bond lengths }[\mathrm{pm}] \text { and angles }\left[^{\circ}\right] \text { of calculated model systems 1-3 (Table 1) } & 1 \\ \text { Calculated }{ }^{29} \text { Si nuclear shieldings of models } Y_{n} M_{3-n} \text { SiX (D-F) (GIAO, SGO) (Tables 2,3) } & 1-2 \\ \text { Standard orientation of 1-6 and of models of type D-F (Tables } 4-52) & 2-29 \\ \text { GIAO shielding tensor for Si of 1-6 and of models of type D-F (Tables } 53-92) & 29-35 \\ \text { IGLO shielding tensor for Si of 1-6 and of models of type D-F (Tables } 93-129) & 35-43 \\ \text { MO contributions to } \sigma \text { for Si of 1-6 and models of type D-F (Tables } 130-169) & 77-82 \\ \text { NBO Analysis for the bonds to silicon of selected models (Tables } 170-189) & 82 \\ \text { Natural Charges on silicon for selected model systems (Table 190) }\end{array}$

*Corresponding authors, e-mail: mail@carsten-strohmann.de, kaupp@mail.uni-wuerzburg.de 
Table 1. Selected bond lengths $[\mathrm{pm}]$ and angles $\left[^{\circ}\right]$ for calculated model systems 1-3. $[6-31+G(d)]^{a}$

\begin{tabular}{|c|c|c|c|c|c|c|}
\hline \multirow{2}{*}{ model } & \multirow{2}{*}{ method } & \multicolumn{3}{|c|}{ bond lengths } & \multicolumn{2}{|c|}{ angles } \\
\hline & & Si-C & $\mathrm{Si}-\mathrm{Li}$ & $\mathrm{Li}-\mathrm{O}$ & C-Si-Li & C-Si-C \\
\hline \multirow{9}{*}{1} & \multirow{3}{*}{ B3LYP } & 194.9 & \multirow{3}{*}{265.7} & 203.1 & 114.5 & 100.4 \\
\hline & & 194.9 & & 204.0 & 118.1 & 100.4 \\
\hline & & 194.9 & & 204.0 & 119.6 & 100.6 \\
\hline & \multirow{4}{*}{ MP2 } & 193.6 & \multirow{3}{*}{260.8} & 200.4 & 116.1 & 100.2 \\
\hline & & 193.7 & & 200.4 & 118.2 & 100.3 \\
\hline & & 193.7 & & 201.2 & 118.3 & 100.4 \\
\hline & & 194.4 & & 206.2 & 115.3 & 100.9 \\
\hline & \multirow[t]{2}{*}{$\mathrm{HF}$} & 194.4 & \multirow[t]{2}{*}{270.9} & 206.3 & 117.3 & 101.0 \\
\hline & & 194.4 & & 207.1 & 118.5 & 101.0 \\
\hline \multirow{9}{*}{2} & \multirow{3}{*}{ B3LYP } & 192.7 & \multirow{3}{*}{251.8} & \multirow{3}{*}{ - } & 114.0 & 104.5 \\
\hline & & 192.7 & & & 114.1 & 104.5 \\
\hline & & 192.7 & & & 114.1 & 104.5 \\
\hline & & 191.6 & \multirow{4}{*}{254.1} & \multirow{4}{*}{ - } & 114.5 & 103.9 \\
\hline & \multirow[t]{3}{*}{ MP2 } & 191.6 & & & 114.6 & 103.9 \\
\hline & & 191.6 & & & 114.6 & 103.9 \\
\hline & & 192.4 & & & 114.3 & 104.3 \\
\hline & \multirow[t]{2}{*}{$\mathrm{HF}$} & 192.4 & \multirow[t]{2}{*}{258.8} & \multirow[t]{2}{*}{-} & 114.3 & 104.3 \\
\hline & & 192.4 & & & 114.3 & 104.3 \\
\hline \multirow{9}{*}{3} & \multirow{3}{*}{ B3LYP } & 197.1 & \multirow{4}{*}{ - } & \multirow{4}{*}{ - } & \multirow{3}{*}{ - } & 97.8 \\
\hline & & 197.1 & & & & 97.8 \\
\hline & & 197.1 & & & & 97.8 \\
\hline & & 195.4 & & & \multirow{4}{*}{ - } & 97.6 \\
\hline & \multirow[t]{3}{*}{ MP2 } & 195.4 & \multirow[t]{3}{*}{ - } & \multirow[t]{3}{*}{ - } & & 97.6 \\
\hline & & 195.4 & & & & 97.6 \\
\hline & & 196.5 & & & & 98.4 \\
\hline & \multirow[t]{2}{*}{$\mathrm{HF}$} & 196.5 & - & - & - & 98.4 \\
\hline & & 196.5 & & & & 98.4 \\
\hline
\end{tabular}

Table 2. Calculated ${ }^{29}$ Si nuclear shieldings for models $\mathrm{Y}_{n} \mathrm{Me}_{3-\mathrm{n}} \mathrm{SiX}^{\mathrm{a}}$

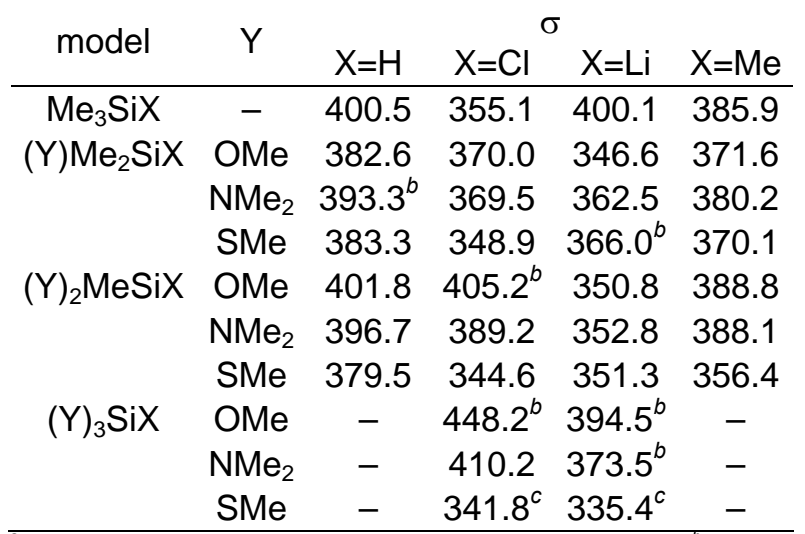

${ }^{a}$ GIAO-HF/6-311+G(2d,p)//B3LYP/6-31+G(d) results ${ }^{b}$ Values from local minimum structure. ${ }^{\circ}$ Optimization started from a conformationally identical model as the local minimum of the corresponding $(\mathrm{MeO})_{3} \mathrm{SiX}$ system. 
Table 3. Calculated ${ }^{29}$ Si nuclear shieldings for models $Y_{n} M_{3-n} S i X^{a}$

\begin{tabular}{cccccc} 
model & $\mathrm{Y}$ & $\mathrm{X}=\mathrm{H}$ & $\mathrm{X}=\mathrm{Cl}$ & $\mathrm{X}=\mathrm{Li}$ & $\mathrm{X}=\mathrm{Me}$ \\
\hline $\mathrm{Me}_{3} \mathrm{SiX}$ & - & 343.5 & 302.2 & 337.7 & 326.3 \\
$(\mathrm{MeY}) \mathrm{Me} 2 \mathrm{SiX}$ & $\mathrm{OMe}$ & 319.4 & 322.3 & 274.6 & 308.8 \\
& $\mathrm{NMe}_{2}$ & $336.6^{b}$ & 325.4 & 295.4 & 322.0 \\
& $\mathrm{SMe}$ & 332.8 & 307.3 & $310.5^{b}$ & 318.4 \\
$(\mathrm{MeY})$ 2MeSiX & $\mathrm{OMe}$ & 343.8 & $365.7^{b}$ & 288.0 & 331.7 \\
& $\mathrm{NMe}_{2}$ & 343.5 & 350.1 & 289.2 & 334.1 \\
& $\mathrm{SMe}$ & 333.8 & 315.4 & 309.7 & 313.8 \\
$(\mathrm{MeY}) 3 \mathrm{SiX}$ & $\mathrm{OMe}$ & - & $417.7^{b}$ & $344.5^{b}$ & - \\
& $\mathrm{NMe}_{2}$ & - & 374.8 & $322.5^{b}$ & - \\
& $\mathrm{SMe}$ & - & $329.2^{c}$ & $301.7^{c}$ & - \\
\hline
\end{tabular}

${ }^{a}$ SGO-BP86/BIII//B3LYP/6-31+G(d) results ${ }^{b}$ Values from local minimum structure. ${ }^{C}$ Optimization started from a conformationally identical model as the local minimum of the corresponding $(\mathrm{MeO})_{3} \mathrm{SiX}$ system.

Table 4. Standard orientation of $1[\mathrm{HF} / 6-31+\mathrm{G}(\mathrm{d})]$

\begin{tabular}{ccccc}
$\begin{array}{c}\text { Center } \\
\text { Number }\end{array}$ & Atom & \multicolumn{3}{c}{ Coordinates (Angstroms) } \\
\hline 1 & Ti & -0.007776 & 0.042273 & 0.122865 \\
2 & $\mathrm{Si}$ & 0.202075 & -0.047655 & 2.822707 \\
3 & $\mathrm{C}$ & 1.577620 & 1.023234 & 3.682553 \\
4 & $\mathrm{C}$ & -1.362783 & 0.464510 & 3.855276 \\
5 & $\mathrm{C}$ & 0.580031 & -1.802746 & 3.567802 \\
6 & $\mathrm{H}$ & 2.574265 & 0.774479 & 3.314699 \\
7 & $\mathrm{H}$ & 1.429466 & 2.089631 & 3.505719 \\
8 & $\mathrm{H}$ & 1.589356 & 0.877473 & 4.763878 \\
9 & $\mathrm{H}$ & 1.524862 & -2.203515 & 3.197432 \\
10 & $\mathrm{H}$ & -0.195483 & -2.525363 & 3.310001 \\
11 & $\mathrm{H}$ & 0.646872 & -1.778294 & 4.656750 \\
12 & $\mathrm{H}$ & -1.197866 & 0.353457 & 4.928249 \\
13 & $\mathrm{H}$ & -1.639118 & 1.505437 & 3.680117 \\
14 & $\mathrm{H}$ & -2.232118 & -0.142814 & 3.599219 \\
15 & $\mathrm{O}$ & -1.904391 & 0.237340 & -0.686043 \\
16 & $\mathrm{C}$ & -2.410785 & -0.326640 & -1.863803 \\
17 & $\mathrm{H}$ & -2.811881 & 0.443685 & -2.517320 \\
18 & $\mathrm{H}$ & -1.599924 & -0.835981 & -2.362849 \\
19 & $\mathrm{H}$ & -3.198179 & -1.041202 & -1.639817 \\
20 & $\mathrm{C}$ & -2.875348 & 0.908494 & 0.081535 \\
21 & $\mathrm{H}$ & -3.299005 & 1.733092 & -0.485052 \\
22 & $\mathrm{H}$ & -3.669064 & 0.225749 & 0.368719 \\
23 & $\mathrm{H}$ & -2.390750 & 1.281505 & 0.969131 \\
24 & $\mathrm{O}$ & 0.605096 & -1.664703 & -0.858297 \\
25 & $\mathrm{C}$ & -0.001023 & -2.840627 & -0.378348 \\
26 & $\mathrm{H}$ & 0.089509 & -3.635765 & -1.113265 \\
27 & $\mathrm{H}$ & 0.452918 & -3.152100 & 0.554991 \\
28 & $\mathrm{H}$ & -1.045178 & -2.626247 & -0.206592 \\
29 & $\mathrm{C}$ & 1.984346 & -1.807200 & -1.084538 \\
30 & $\mathrm{H}$ & 2.167937 & -2.580079 & -1.825621 \\
31 & $\mathrm{H}$ & 2.355855 & -0.862259 & -1.450137 \\
32 & $\mathrm{H}$ & 2.498141 & -2.063007 & -0.163552 \\
33 & $\mathrm{O}$ & 1.043868 & 1.506023 & -0.881087 \\
34 & $\mathrm{C}$ & 1.794875 & 2.445103 & -0.147463 \\
35 & $\mathrm{H}$ & 2.745650 & 2.630606 & -0.639145 \\
36 & $\mathrm{H}$ & 1.249515 & 3.380074 & -0.058850 \\
37 & $\mathrm{H}$ & 1.962277 & 2.033642 & 0.833964
\end{tabular}




\begin{tabular}{lllll}
38 & $\mathrm{C}$ & 0.750932 & 1.918293 & -2.186706 \\
39 & $\mathrm{H}$ & 1.664295 & 2.130957 & -2.735600 \\
40 & $\mathrm{H}$ & 0.221295 & 1.115904 & -2.678351 \\
41 & $\mathrm{H}$ & 0.127511 & 2.808080 & -2.179899 \\
\hline
\end{tabular}

Total Energy of $1[\mathrm{HF} / 6-31+\mathrm{G}(\mathrm{d})]: \mathrm{E}(\mathrm{RHF})=-877.476378165$ Hartree Corrected Zero Point Energy: -877.095789 Hartree

Table 5. Standard orientation of $1[B 3 L Y P / 6-31+G(d)]$

\begin{tabular}{ccccc}
$\begin{array}{c}\text { Center } \\
\text { Number }\end{array}$ & $\begin{array}{c}\text { Atom } \\
\text { Type }\end{array}$ & \multicolumn{3}{c}{ Coordinates (Angstroms) } \\
\hline 1 & $\mathrm{Li}$ & -0.041438 & 0.043312 & 0.106869 \\
2 & $\mathrm{Si}$ & 0.222944 & -0.027053 & 2.749627 \\
3 & $\mathrm{C}$ & 1.571119 & 1.085689 & 3.611166 \\
4 & $\mathrm{C}$ & -1.335795 & 0.401148 & 3.838844 \\
5 & $\mathrm{C}$ & 0.695801 & -1.775771 & 3.469570 \\
6 & $\mathrm{H}$ & 2.580441 & 0.881887 & 3.222585 \\
7 & $\mathrm{H}$ & 1.374778 & 2.158104 & 3.459732 \\
8 & $\mathrm{H}$ & 1.600944 & 0.913183 & 4.699214 \\
9 & $\mathrm{H}$ & 1.661222 & -2.127890 & 3.075897 \\
10 & $\mathrm{H}$ & -0.055385 & -2.539164 & 3.216606 \\
11 & $\mathrm{H}$ & 0.780618 & -1.754348 & 4.568268 \\
12 & $\mathrm{H}$ & -1.126664 & 0.273240 & 4.913308 \\
13 & $\mathrm{H}$ & -1.660606 & 1.442910 & 3.694657 \\
14 & $\mathrm{H}$ & -2.193967 & -0.243446 & 3.595770 \\
15 & $\mathrm{O}$ & -1.926218 & 0.292312 & -0.608083 \\
16 & $\mathrm{C}$ & -2.415849 & 0.045853 & -1.918996 \\
17 & $\mathrm{H}$ & -3.339651 & -0.549623 & -1.883236 \\
18 & $\mathrm{H}$ & -2.620725 & 0.989575 & -2.445824 \\
19 & $\mathrm{H}$ & -1.644976 & -0.514349 & -2.453048 \\
20 & $\mathrm{C}$ & -2.866638 & 0.979346 & 0.221117 \\
21 & $\mathrm{H}$ & -3.795005 & 0.397533 & 0.306386 \\
22 & $\mathrm{H}$ & -2.402080 & 1.080472 & 1.203007 \\
23 & $\mathrm{H}$ & -3.093496 & 1.970496 & -0.196997 \\
24 & $\mathrm{O}$ & 0.440283 & -1.697624 & -0.840450 \\
25 & $\mathrm{C}$ & -0.330861 & -2.816860 & -0.398120 \\
26 & $\mathrm{H}$ & -0.224662 & -3.652905 & -1.104057 \\
27 & $\mathrm{H}$ & -0.014150 & -3.131689 & 0.603915 \\
28 & $\mathrm{H}$ & -1.372921 & -2.494088 & -0.360906 \\
29 & $\mathrm{C}$ & 1.836060 & -1.993754 & -0.888306 \\
30 & $\mathrm{H}$ & 2.028215 & -2.809099 & -1.600143 \\
31 & $\mathrm{H}$ & 2.344890 & -1.086485 & -1.218458 \\
32 & $\mathrm{H}$ & 2.204487 & -2.277171 & 0.106338 \\
33 & $\mathrm{O}$ & 1.139532 & 1.370739 & -0.895111 \\
34 & $\mathrm{C}$ & 1.824328 & 2.374401 & -0.140436 \\
35 & $\mathrm{H}$ & 1.311969 & 3.342065 & -0.236944 \\
36 & $\mathrm{H}$ & 1.807464 & 2.048987 & 0.900235 \\
37 & $\mathrm{H}$ & 2.860720 & 2.472659 & -0.493276 \\
38 & $\mathrm{C}$ & 1.061292 & 1.677667 & -2.280269 \\
39 & $\mathrm{H}$ & 0.484536 & 2.599305 & -2.446204 \\
40 & $\mathrm{H}$ & 2.066174 & 1.802935 & -2.708932 \\
41 & $\mathrm{H}$ & 0.561164 & 0.840343 & -2.772044 \\
\hline & & & & \\
\hline & & & \\
13 & &
\end{tabular}

Total Energy of 1 [B3LYP/6-31+G(d)]: E(RB+HF-LYP $)=-881.933944578$ Hartree Corrected Zero Point Energy: -881.577469 Hartree

Table 6. Standard orientation of $1[\mathrm{MP} 2 / 6-31+\mathrm{G}(\mathrm{d})]$ 


\begin{tabular}{ccccc}
$\begin{array}{c}\text { Center } \\
\text { Number }\end{array}$ & $\begin{array}{c}\text { Atom } \\
\text { Type }\end{array}$ & \multicolumn{3}{c}{ Coordinates (Angstroms) } \\
\hline 1 & Li & -0.440571 & 0.055742 & -0.052224 \\
2 & $\mathrm{Si}$ & 2.162504 & 0.053813 & 0.106026 \\
3 & $\mathrm{C}$ & 3.112294 & 1.677883 & -0.351563 \\
4 & $\mathrm{C}$ & 2.974785 & -0.353063 & 1.816380 \\
5 & $\mathrm{C}$ & 3.083060 & -1.221737 & -1.024899 \\
6 & $\mathrm{H}$ & 2.887384 & 2.012316 & -1.373783 \\
7 & $\mathrm{H}$ & 2.859339 & 2.504902 & 0.326490 \\
8 & $\mathrm{H}$ & 4.200434 & 1.526118 & -0.287444 \\
9 & $\mathrm{H}$ & 2.862544 & -1.063614 & -2.089845 \\
10 & $\mathrm{H}$ & 2.804476 & -2.256830 & -0.782868 \\
11 & $\mathrm{H}$ & 4.173627 & -1.139556 & -0.901066 \\
12 & $\mathrm{H}$ & 4.072521 & -0.358372 & 1.737891 \\
13 & $\mathrm{H}$ & 2.707018 & 0.386056 & 2.584443 \\
14 & $\mathrm{H}$ & 2.668013 & -1.339501 & 2.190984 \\
15 & $\mathrm{O}$ & -1.498157 & -0.616829 & 1.511504 \\
16 & $\mathrm{C}$ & -2.725752 & -1.344672 & 1.507702 \\
17 & $\mathrm{H}$ & -2.554757 & -2.387661 & 1.804238 \\
18 & $\mathrm{H}$ & -3.444955 & -0.888498 & 2.200047 \\
19 & $\mathrm{H}$ & -3.110595 & -1.310247 & 0.488323 \\
20 & $\mathrm{C}$ & -0.844641 & -0.675452 & 2.787601 \\
21 & $\mathrm{H}$ & -0.631408 & -1.717844 & 3.053490 \\
22 & $\mathrm{H}$ & 0.090740 & -0.127154 & 2.684899 \\
23 & $\mathrm{H}$ & -1.480308 & -0.220308 & 3.557344 \\
24 & $\mathrm{O}$ & -1.276108 & -1.113554 & -1.460502 \\
25 & $\mathrm{C}$ & -0.753788 & -2.447770 & -1.361983 \\
26 & $\mathrm{H}$ & -1.298760 & -3.113794 & -2.042550 \\
27 & $\mathrm{H}$ & 0.315131 & -2.455353 & -1.599202 \\
28 & $\mathrm{H}$ & -0.899231 & -2.767796 & -0.329833 \\
29 & $\mathrm{C}$ & -1.062048 & -0.576216 & -2.774177 \\
30 & $\mathrm{H}$ & -1.582846 & -1.190157 & -3.519256 \\
31 & $\mathrm{H}$ & -1.466168 & 0.435621 & -2.768745 \\
32 & $\mathrm{H}$ & 0.010360 & -0.544853 & -2.999453 \\
33 & $\mathrm{O}$ & -1.368589 & 1.771751 & -0.509612 \\
34 & $\mathrm{C}$ & -0.687424 & 2.975740 & -0.128027 \\
35 & $\mathrm{H}$ & -0.830246 & 3.169751 & 0.942555 \\
36 & $\mathrm{H}$ & 0.368920 & 2.813834 & -0.336191 \\
37 & $\mathrm{H}$ & -1.072298 & 3.821722 & -0.710408 \\
38 & $\mathrm{C}$ & -2.773992 & 1.857622 & -0.274934 \\
39 & $\mathrm{H}$ & -2.981556 & 1.956592 & 0.798083 \\
40 & $\mathrm{H}$ & -3.199327 & 2.715510 & -0.810619 \\
41 & $\mathrm{H}$ & -3.213504 & 0.933546 & -0.652952 \\
\hline & & & & \\
\hline & & & & \\
10 & &
\end{tabular}

Total Energy of 1 [MP2/6-31+G(d)]: EUMP2 = -879.33428849995 Hartree Corrected Zero Point Energy: Could not be determined, but molecule has same conformation as results from B3LYP and HF calculations.

Table 7. Standard orientation of $2[\mathrm{HF} / 6-31+\mathrm{G}(\mathrm{d})]$

\begin{tabular}{ccccc}
$\begin{array}{c}\text { Center } \\
\text { Number }\end{array}$ & $\begin{array}{c}\text { Atom } \\
\text { Type }\end{array}$ & \multicolumn{3}{c}{ Coordinates (Angstroms) } \\
\hline 1 & $\mathrm{Si}$ & 0.000462 & -0.000363 & 0.346411 \\
2 & $\mathrm{Li}$ & 0.000806 & -0.003593 & 2.904522 \\
3 & $\mathrm{C}$ & -1.260088 & -1.218528 & -0.445509 \\
4 & $\mathrm{C}$ & -0.425430 & 1.701550 & -0.442337 \\
5 & $\mathrm{C}$ & 1.685889 & -0.481230 & -0.446102 \\
6 & $\mathrm{H}$ & -1.218809 & -1.177300 & -1.534424 \\
7 & $\mathrm{H}$ & -2.282541 & -0.988558 & -0.150089 \\
8 & $\mathrm{H}$ & -1.064024 & -2.248451 & -0.151599
\end{tabular}




\begin{tabular}{ccccc}
9 & $\mathrm{H}$ & 0.285440 & 2.471523 & -0.146735 \\
10 & $\mathrm{H}$ & -1.414961 & 2.046372 & -0.146558 \\
11 & $\mathrm{H}$ & -0.411845 & 1.646812 & -1.531354 \\
12 & $\mathrm{H}$ & 1.629688 & -0.463816 & -1.534988 \\
13 & $\mathrm{H}$ & 1.997882 & -1.482311 & -0.152730 \\
14 & $\mathrm{H}$ & 2.479770 & 0.202886 & -0.150642 \\
\hline
\end{tabular}

Total Energy of $2[H F / 6-31+G(d)]: E(R H F)=-415.206817458$ Hartree Corrected Zero Point Energy: -415.088860 Hartree

Table 8. Standard orientation of 2 [B3LYP/6-31+G(d)]

\begin{tabular}{ccccc}
$\begin{array}{c}\text { Center } \\
\text { Number }\end{array}$ & Atom & \multicolumn{3}{c}{ Coordinates (Angstroms) } \\
\hline 1 & $\mathrm{Si}$ & -0.000007 & -0.000474 & 0.344877 \\
2 & $\mathrm{Li}$ & -0.004215 & -0.003259 & 2.862381 \\
3 & $\mathrm{C}$ & -1.263310 & -1.223610 & -0.443852 \\
4 & $\mathrm{C}$ & -0.428278 & 1.706558 & -0.440718 \\
5 & $\mathrm{C}$ & 1.692845 & -0.481413 & -0.440634 \\
6 & $\mathrm{H}$ & -1.224347 & -1.182609 & -1.543445 \\
7 & $\mathrm{H}$ & -2.293164 & -0.990288 & -0.141163 \\
8 & $\mathrm{H}$ & -1.062408 & -2.261258 & -0.144666 \\
9 & $\mathrm{H}$ & 0.303507 & 2.475384 & -0.157665 \\
10 & $\mathrm{H}$ & -1.416521 & 2.063068 & -0.120115 \\
11 & $\mathrm{H}$ & -0.438175 & 1.648063 & -1.540195 \\
12 & $\mathrm{H}$ & 1.644097 & -0.455838 & -1.540304 \\
13 & $\mathrm{H}$ & 2.000343 & -1.494355 & -0.147329 \\
14 & $\mathrm{H}$ & 2.491873 & 0.205025 & -0.129306 \\
\hline
\end{tabular}

Total Energy of 2 [B3LYP/6-31+G(d)]: E(RB+HF-LYP) $=-416.770643918$ Hartree Corrected Zero Point Energy: -416.659456 Hartree

Table 9. Standard orientation of $2[\mathrm{MP} 2 / 6-31+\mathrm{G}(\mathrm{d})]$

\begin{tabular}{ccccc}
$\begin{array}{c}\text { Center } \\
\text { Number }\end{array}$ & Atom & \multicolumn{3}{c}{ Coordinates (Angstroms) } \\
\hline 1 & $\mathrm{Si}$ & 0.000323 & 0.000300 & 0.351070 \\
2 & $\mathrm{Li}$ & 0.003301 & 0.001688 & 2.891888 \\
3 & $\mathrm{C}$ & 1.581885 & -0.731181 & -0.446621 \\
4 & $\mathrm{C}$ & -1.424718 & -1.004987 & -0.443493 \\
5 & $\mathrm{C}$ & -0.158586 & 1.735218 & -0.447317 \\
6 & $\mathrm{H}$ & 1.505419 & -0.696353 & -1.542752 \\
7 & $\mathrm{H}$ & 1.738253 & -1.777404 & -0.157386 \\
8 & $\mathrm{H}$ & 2.480265 & -0.172281 & -0.158133 \\
9 & $\mathrm{H}$ & -2.408492 & -0.616014 & -0.154356 \\
10 & $\mathrm{H}$ & -1.390414 & -2.061877 & -0.152864 \\
11 & $\mathrm{H}$ & -1.357071 & -0.958449 & -1.539767 \\
12 & $\mathrm{H}$ & -0.152842 & 1.650886 & -1.543404 \\
13 & $\mathrm{H}$ & 0.670045 & 2.393743 & -0.160240 \\
14 & $\mathrm{H}$ & -1.091068 & 2.234178 & -0.157165 \\
\hline
\end{tabular}

Total Energy of 2 [MP2/6-31+G(d)]: E(UMP2) -415.70363835407 Hartree Corrected Zero Point Energy: -415.589878 Hartree

Table 10. Standard orientation of $3[\mathrm{HF} / 6-31+\mathrm{G}(\mathrm{d})]$

\begin{tabular}{cccc} 
Center & Atom & \multicolumn{2}{c}{ Coordinates (Angstroms) } \\
Number & Type & $\mathrm{X}$ \\
\hline
\end{tabular}




\begin{tabular}{ccccc}
\hline 1 & $\mathrm{Si}$ & 0.001422 & -0.000631 & 0.515281 \\
2 & $\mathrm{C}$ & -1.233907 & -1.193400 & -0.439269 \\
3 & $\mathrm{C}$ & -0.416120 & 1.666605 & -0.436499 \\
4 & $\mathrm{C}$ & 1.651601 & -0.471506 & -0.441326 \\
5 & $\mathrm{H}$ & -1.144948 & -1.105094 & -1.527838 \\
6 & $\mathrm{H}$ & -2.270970 & -0.977686 & -0.175650 \\
7 & $\mathrm{H}$ & -1.052359 & -2.237640 & -0.178264 \\
8 & $\mathrm{H}$ & 0.290235 & 2.456298 & -0.173853 \\
9 & $\mathrm{H}$ & -1.410408 & 2.031663 & -0.172609 \\
10 & $\mathrm{H}$ & -0.386923 & 1.546515 & -1.525269 \\
11 & $\mathrm{H}$ & 1.530128 & -0.435643 & -1.529742 \\
12 & $\mathrm{H}$ & 1.983317 & -1.478245 & -0.180606 \\
13 & $\mathrm{H}$ & 2.465385 & 0.207016 & -0.178869 \\
\hline
\end{tabular}

Total Energy of $3[\mathrm{HF} / 6-31+\mathrm{G}(\mathrm{d})]$ : $\mathrm{E}(\mathrm{RHF})=-407.733331726$ Hartree Corrected Zero Point Energy: -407.619049 Hartree

Table 11. Standard orientation of $3[B 3 L Y P / 6-31+G(d)]$

\begin{tabular}{ccccc}
$\begin{array}{c}\text { Center } \\
\text { Number }\end{array}$ & Atom & \multicolumn{3}{c}{ Coordinates (Angstroms) } \\
\hline 1 & $\mathrm{Si}$ & $\mathbf{X}$ & $\mathrm{Y}$ & $\mathrm{Z}$ \\
\hline 2 & $\mathrm{C}$ & -1.233417 & -0.000918 & 0.533064 \\
3 & $\mathrm{C}$ & -0.415612 & 1.664697 & -0.436003 \\
4 & $\mathrm{C}$ & 1.650891 & -0.470825 & -0.434943 \\
5 & $\mathrm{H}$ & -1.135329 & -1.093920 & -1.535123 \\
6 & $\mathrm{H}$ & -2.280317 & -0.973802 & -0.172962 \\
7 & $\mathrm{H}$ & -1.050974 & -2.247022 & -0.176944 \\
8 & $\mathrm{H}$ & 0.294640 & 2.464298 & -0.172192 \\
9 & $\mathrm{H}$ & -1.421399 & 2.031483 & -0.176627 \\
10 & $\mathrm{H}$ & -0.378706 & 1.529942 & -1.533969 \\
11 & $\mathrm{H}$ & 1.516148 & -0.425812 & -1.538471 \\
12 & $\mathrm{H}$ & 1.984224 & -1.489092 & -0.184970 \\
13 & $\mathrm{H}$ & 2.473983 & 0.211501 & -0.175634 \\
\hline
\end{tabular}

Total Energy of 3 [B3LYP/6-31+G(d)]: E(RB+HF-LYP) $=-409.248441736$ Hartree Corrected Zero Point Energy: -409.141115 Hartree

Table 12. Standard orientation of $3[M P 2 / 6-31+G(d)]$

\begin{tabular}{ccccc}
$\begin{array}{c}\text { Center } \\
\text { Number }\end{array}$ & Atom & \multicolumn{3}{c}{ Coordinates (Angstroms) } \\
\hline 1 & $\mathrm{Si}$ & -0.000597 & 0.000817 & -0.679879 \\
2 & $\mathrm{C}$ & -1.532347 & -0.731936 & 0.287137 \\
3 & $\mathrm{C}$ & 1.400235 & -0.961563 & 0.284384 \\
4 & $\mathrm{C}$ & 0.132852 & 1.692392 & 0.289185 \\
5 & $\mathrm{H}$ & -1.382938 & -0.661600 & 1.379981 \\
6 & $\mathrm{H}$ & -1.695592 & -1.789292 & 0.035360 \\
7 & $\mathrm{H}$ & -2.457771 & -0.194379 & 0.036861 \\
8 & $\mathrm{H}$ & 2.397117 & -0.572324 & 0.033818 \\
9 & $\mathrm{H}$ & 1.398701 & -2.031031 & 0.030950 \\
10 & $\mathrm{H}$ & 1.264436 & -0.870726 & 1.377491 \\
11 & $\mathrm{H}$ & 0.121836 & 1.526295 & 1.381827 \\
12 & $\mathrm{H}$ & -0.702122 & 2.362455 & 0.040418 \\
13 & $\mathrm{H}$ & 1.060255 & 2.225795 & 0.037361 \\
\hline
\end{tabular}

Total Energy of 3 [MP2/6-31+G(d)]: E(UMP2) $=-408.22999184777$ Hartree Corrected Zero Point Energy: - -408.120105 Hartree 
Table 13. Standard orientation of 4.3 THF [B3LYP/TZVP]

\begin{tabular}{|c|c|c|c|c|}
\hline & Atom & & oordinates (Angstroms) & \\
\hline Number & Type & $x$ & $\mathrm{Y}$ & Z \\
\hline 1 & C & 5.64196474886706 & 3.10977594152814 & 5.30733089982488 \\
\hline 2 & C & 4.35484254969822 & 1.32579554820701 & 3.86247843515848 \\
\hline 3 & C & 4.02186311433392 & 1.59734569113013 & 1.24011481708417 \\
\hline 4 & C & 5.06169763249479 & 3.78324885546995 & 0.14981947587394 \\
\hline 5 & C & 6.35708968444834 & 5.58151477276480 & 1.57252341078541 \\
\hline 6 & C & 6.65090362323161 & 5.25853397138657 & 4.16770904449721 \\
\hline 7 & $\mathrm{Si}$ & 1.87022056764295 & -0.68301125244410 & -0.68911571851852 \\
\hline 8 & C & 3.06200248906867 & -4.00207779383787 & 0.46546411228460 \\
\hline 9 & C & 1.42942168612001 & -6.08075215972531 & 0.24537510061757 \\
\hline 10 & C & 2.14073221508473 & -8.51883314729119 & 0.94036091495460 \\
\hline 11 & C & 4.54720745091577 & -8.95558262946068 & 1.90792187681082 \\
\hline 12 & C & 6.21347560045551 & -6.93499883464966 & 2.15826411263421 \\
\hline 13 & C & 5.47408123284833 & -4.50944473964300 & 1.44730152170928 \\
\hline 14 & $\mathrm{~N}$ & 3.09806457287243 & -0.31675645337647 & -3.86509755029632 \\
\hline 15 & C & 5.73622268836544 & -0.90134442086548 & -4.44487720492846 \\
\hline 16 & C & 188378 & 35252772146 & -6.4 \\
\hline 17 & C & 31050054430 & -0.47483201896290 & -5.99615594616484 \\
\hline 18 & C & 1.05040831873394 & -3.10161140816892 & -7.19647319071084 \\
\hline 19 & $\mathrm{Li}$ & -2.92694299113344 & 0.74329441480377 & 0.33255291154660 \\
\hline 20 & $\mathrm{O}$ & 725 & -0.4 & -1.6 \\
\hline 21 & C & -6.00545694741867 & -2.36171843591853 & -3.62807833153502 \\
\hline 22 & C & -8.67588218304294 & -3.44009244812377 & -3.58727045022032 \\
\hline 23 & C & -10.2 & 870 & -2.9 \\
\hline 24 & C & -8.5 & 1418 & 9613 \\
\hline 25 & $\mathrm{O}$ & -3.29463746945033 & 76829 & 0.00663458559962 \\
\hline 26 & C & 3560176654 & 10350 & -2.52141318975899 \\
\hline 27 & C & -1.3 & 7752 & 12941 \\
\hline 28 & C & -1.31392297727788 & 8.61973571094504 & -0.01673757648185 \\
\hline 29 & C & -1.84844504516333 & 6.28165646185305 & 1.59642363072632 \\
\hline 30 & $\mathrm{O}$ & -4.6 & 1036 & 141 \\
\hline 31 & C & -4.3 & 671 & 6950 \\
\hline 32 & C & -2.99138650775947 & -3.56079495 & 6.80275483415444 \\
\hline 33 & C & -3.30347437237445 & -1.07665723 & 8.26725674555563 \\
\hline 34 & C & $-3 .($ & 0. & 095 \\
\hline 35 & $\mathrm{H}$ & -0.31267057457698 & -3.03061969893785 & -8.75262590586341 \\
\hline 36 & $\mathrm{H}$ & 2.82847134805834 & -3.81340872868304 & -7.96583944920369 \\
\hline 37 & $\mathrm{H}$ & -9.2 & -4 & 5730236 \\
\hline 38 & $\mathrm{H}$ & 42 & 86 & 36052 \\
\hline 39 & $\mathrm{H}$ & -10.59312871199391 & 0.04397907675073 & -4.58858206031101 \\
\hline 40 & $\mathrm{H}$ & & $-4.47>-2$ & 186806073283 \\
\hline 41 & $\mathrm{H}$ & & & 676 \\
\hline 42 & $\mathrm{H}$ & 303 & 16846841634 & -5.44310920343361 \\
\hline 43 & $\mathrm{H}$ & 07584648761 & 5415235940 & -6.67290907965277 \\
\hline 44 & $\mathrm{H}$ & & & -4.98192945381095 \\
\hline 45 & $\mathrm{H}$ & & 0.1 & -5.3 \\
\hline 46 & $\mathrm{H}$ & -8.84749448940717 & -4.88694216388356 & -2.12327513639541 \\
\hline 47 & $\mathrm{H}$ & -12.07709225563995 & -1.56587298350207 & -2.07143346680550 \\
\hline 48 & $\mathrm{H}$ & & & 2844681982 \\
\hline 49 & $\mathrm{H}$ & -4.5 & -3.72713294638777 & -3.21469692063842 \\
\hline 50 & $\mathrm{H}$ & -8.65263708188437 & 2.35386730285157 & -1.21195927410068 \\
\hline 51 & $\mathrm{H}$ & -3.27431854656636 & 4.02571406770544 & -3.85253738342789 \\
\hline 52 & $\mathrm{H}$ & & -0.65236898308515 & -2.71022092386684 \\
\hline 53 & $\mathrm{H}$ & -8.90149748339132 & -0.22479928026308 & 0.91108573760267 \\
\hline 54 & $\mathrm{H}$ & -5.34595524094041 & 6.44334951988416 & -2.76588268832272 \\
\hline 55 & $\mathrm{H}$ & 0.82071616927066 & -10.07474768051975 & 0.73264646229603 \\
\hline 56 & $\mathrm{H}$ & -0.46012487026362 & -5.78273497569132 & -0.50581865425534 \\
\hline 57 & $\mathrm{H}$ & 0.40938847405092 & 6.63041222743601 & -3.16793265147817 \\
\hline
\end{tabular}




\begin{tabular}{ccccc}
58 & $\mathrm{H}$ & 5.11737185015585 & -10.84486133274074 & 2.45951206189753 \\
59 & $\mathrm{H}$ & -1.76975752820961 & 8.97910978559995 & -4.14499817266594 \\
60 & $\mathrm{H}$ & 4.85933369244316 & 4.06204373109020 & -1.87180348322758 \\
61 & $\mathrm{H}$ & 8.09782846423232 & -7.25042413264544 & 2.90400270369426 \\
62 & $\mathrm{H}$ & -3.60043167611625 & -4.00950796614689 & 2.72122064420609 \\
63 & $\mathrm{H}$ & 6.81369973111351 & -2.97452759452969 & 1.67268848545892 \\
64 & $\mathrm{H}$ & -6.39446214142161 & -3.35628076814094 & 4.48524752768344 \\
65 & $\mathrm{H}$ & 0.49458482760471 & 9.47989590633881 & 0.46419824655752 \\
66 & $\mathrm{H}$ & -2.79310990847241 & 10.04025739429020 & 0.23319867868302 \\
67 & $\mathrm{H}$ & 7.15760654566830 & 7.23069818332464 & 0.65171711298535 \\
68 & $\mathrm{H}$ & -0.99959930556411 & -3.94751004470313 & 6.42996275634065 \\
69 & $\mathrm{H}$ & -3.79381637781196 & -5.18724085540479 & 7.78119540013616 \\
70 & $\mathrm{H}$ & -0.10508791402210 & 5.32620883767008 & 2.14717593596362 \\
71 & $\mathrm{H}$ & -2.96766937592962 & 6.69792578464255 & 3.27870934091875 \\
72 & $\mathrm{H}$ & 3.61613627201651 & -0.34396409546406 & 4.80082275794027 \\
73 & $\mathrm{H}$ & -1.11904689470309 & 1.46772522106248 & 5.86707426890433 \\
74 & $\mathrm{H}$ & -4.22385180725744 & 2.58447490427004 & 6.51418933190621 \\
75 & $\mathrm{H}$ & -5.16531895191535 & -0.99099669368076 & 9.15781720534341 \\
76 & $\mathrm{H}$ & 7.66457186348995 & 6.64712260781820 & 5.28196686753014 \\
77 & $\mathrm{H}$ & -1.87934339155771 & -0.81538555080023 & 9.73313138794033 \\
78 & $\mathrm{H}$ & 5.87740077534272 & 2.81644320910369 & 7.32367557245343 \\
\hline
\end{tabular}

Total Energy of 4.3 THF [B3LYP/TZVP]: E(SCF) $=-1670.4880514110$ Hartree

Table 14. Standard orientation of 5.3 THF [B3LYP/TZVP]

\begin{tabular}{ccccc}
$\begin{array}{c}\text { Center } \\
\text { Number }\end{array}$ & $\begin{array}{c}\text { Atom } \\
\text { Type }\end{array}$ & \multicolumn{3}{c}{ Coordinates (Angstroms) } \\
\hline 1 & $\mathrm{H}$ & -3.369322 & -0.229269 & -4.054623 \\
2 & $\mathrm{H}$ & -4.783438 & 0.419387 & -3.229079 \\
3 & $\mathrm{H}$ & 0.537682 & 3.709238 & -3.664387 \\
4 & $\mathrm{H}$ & -1.315185 & -0.919646 & -3.602877 \\
5 & $\mathrm{C}$ & -3.697490 & 0.305781 & -3.160010 \\
6 & $\mathrm{H}$ & -0.861595 & -3.329218 & -3.405080 \\
7 & $\mathrm{H}$ & -3.250679 & 1.302777 & -3.180596 \\
8 & $\mathrm{H}$ & 1.177060 & 1.361835 & -3.547341 \\
9 & $\mathrm{H}$ & -2.515756 & -3.035115 & -2.875403 \\
10 & $\mathrm{H}$ & 2.958487 & 3.490279 & -3.419446 \\
11 & $\mathrm{H}$ & 3.740962 & -3.466199 & -2.786037 \\
12 & $\mathrm{H}$ & 2.056690 & -2.960971 & -2.870657 \\
13 & $\mathrm{C}$ & -1.468157 & -2.836342 & -2.637264 \\
14 & $\mathrm{C}$ & 0.912197 & 3.348433 & -2.705754 \\
15 & $\mathrm{C}$ & -1.162735 & -1.329754 & -2.594840 \\
16 & $\mathrm{H}$ & 2.906866 & -0.733299 & -2.592958 \\
17 & $\mathrm{H}$ & -3.821489 & -1.419462 & -1.886661 \\
18 & $\mathrm{H}$ & -0.095182 & -1.208385 & -2.376259 \\
19 & $\mathrm{C}$ & 0.879010 & 1.830183 & -2.602017 \\
20 & $\mathrm{C}$ & 2.953082 & -2.929846 & -2.253758 \\
21 & $\mathrm{H}$ & -0.073038 & 1.410664 & -2.280968 \\
22 & $\mathrm{H}$ & 0.309415 & 3.792095 & -1.910209 \\
23 & $\mathrm{C}$ & -3.328253 & -0.434341 & -1.866491 \\
24 & $\mathrm{C}$ & 2.402196 & 3.645964 & -2.490481 \\
25 & $\mathrm{H}$ & 4.465873 & -1.362756 & -2.021357 \\
26 & $\mathrm{H}$ & -1.250629 & -3.303341 & -1.673960 \\
27 & $\mathrm{C}$ & 3.376932 & -1.479178 & -1.957337 \\
28 & $\mathrm{H}$ & 2.595020 & 4.663383 & -2.149754 \\
29 & $\mathrm{~N}$ & -1.894208 & -0.547247 & -1.607436 \\
30 & $\mathrm{H}$ & 3.815046 & 2.220449 & -1.579715 \\
31 & $\mathrm{H}$ & -3.775734 & 0.105186 & -1.030469 \\
32 & $\mathrm{O}$ & 1.866914 & 1.504810 & -1.588955
\end{tabular}




\begin{tabular}{llccc}
33 & $\mathrm{C}$ & 2.800529 & 2.602116 & -1.442991 \\
34 & $\mathrm{H}$ & -2.151595 & 2.220108 & -1.211795 \\
35 & $\mathrm{C}$ & 2.676361 & -3.537928 & -0.853147 \\
36 & $\mathrm{H}$ & 3.206389 & -4.475743 & -0.687565 \\
37 & $\mathrm{H}$ & -3.740281 & -3.043621 & -0.183649 \\
38 & $\mathrm{H}$ & 1.610204 & -3.723904 & -0.722376 \\
39 & $\mathrm{H}$ & -3.060008 & 4.331422 & -0.345991 \\
40 & $\mathrm{O}$ & 2.960581 & -1.213224 & -0.606050 \\
41 & $\mathrm{H}$ & 2.704442 & 2.991238 & -0.426070 \\
42 & $\mathrm{C}$ & -2.336525 & 2.315696 & -0.147099 \\
43 & $\mathrm{Si}$ & -1.137656 & -0.369508 & 0.047611 \\
44 & $\mathrm{H}$ & -4.897916 & -3.080950 & 1.155876 \\
45 & $\mathrm{C}$ & -3.833154 & -3.055178 & 0.904002 \\
46 & $\mathrm{Li}$ & 1.505111 & 0.153388 & -0.128438 \\
47 & $\mathrm{C}$ & 3.139890 & -2.443894 & 0.112474 \\
48 & $\mathrm{C}$ & -2.850392 & 3.515549 & 0.338770 \\
49 & $\mathrm{H}$ & -3.389833 & -3.985264 & 1.268768 \\
50 & $\mathrm{H}$ & 4.200278 & -2.559556 & 0.370414 \\
51 & $\mathrm{C}$ & -2.064932 & 1.220702 & 0.690817 \\
52 & $\mathrm{H}$ & -1.149033 & -3.661160 & 1.233581 \\
53 & $\mathrm{H}$ & -3.714511 & -0.928833 & 1.199083 \\
54 & $\mathrm{H}$ & 2.555024 & -2.380526 & 1.028738 \\
55 & $\mathrm{~N}$ & -1.759860 & -1.621054 & 1.208542 \\
56 & $\mathrm{C}$ & -3.172039 & -1.816112 & 1.526587 \\
57 & $\mathrm{H}$ & 0.120398 & -2.454489 & 1.275785 \\
58 & $\mathrm{C}$ & -0.877193 & -2.681407 & 1.663613 \\
59 & $\mathrm{C}$ & -3.107985 & 3.666966 & 1.698906 \\
60 & $\mathrm{H}$ & -3.511856 & 4.597114 & 2.082072 \\
61 & $\mathrm{H}$ & 4.236253 & 0.204018 & 1.426930 \\
62 & $\mathrm{O}$ & 2.299919 & 0.857725 & 1.587957 \\
63 & $\mathrm{C}$ & -2.335753 & 1.406588 & 2.056985 \\
64 & $\mathrm{H}$ & 4.063796 & 1.959975 & 1.593097 \\
65 & $\mathrm{H}$ & -3.323315 & -1.861906 & 2.616110 \\
66 & $\mathrm{C}$ & 3.692823 & 0.993489 & 1.946215 \\
67 & $\mathrm{C}$ & -2.846487 & 2.602959 & 2.557440 \\
68 & $\mathrm{H}$ & -2.152108 & 0.585763 & 2.743301 \\
69 & $\mathrm{C}$ & -0.784363 & -2.843157 & 3.187476 \\
70 & $\mathrm{H}$ & 0.545831 & 0.549064 & 2.602166 \\
71 & $\mathrm{H}$ & -0.064303 & -3.626156 & 3.447932 \\
72 & $\mathrm{H}$ & -1.742331 & -3.126009 & 3.629925 \\
73 & $\mathrm{C}$ & 1.499475 & 1.041900 & 2.776104 \\
74 & $\mathrm{H}$ & -3.049651 & 2.700882 & 3.619088 \\
75 & $\mathrm{H}$ & -0.465454 & -1.908445 & 3.657185 \\
76 & $\mathrm{H}$ & 1.320414 & 2.111974 & 2.935975 \\
77 & $\mathrm{C}$ & 3.768407 & 0.879983 & 3.483738 \\
78 & $\mathrm{H}$ & 4.534608 & 0.174469 & 3.806732 \\
79 & $\mathrm{H}$ & 2.259104 & -0.647368 & 3.874432 \\
80 & $\mathrm{C}$ & 2.349231 & 0.441118 & 3.886802 \\
81 & $\mathrm{H}$ & 4.006269 & 1.849770 & 3.926205 \\
82 & $\mathrm{H}$ & 2.061105 & 0.795319 & 4.876845 \\
\hline & & & & \\
\hline 5 & &
\end{tabular}

Total Energy of 5.3 THF [B3LYP/TZVP]: E(SCF) $=-1652.0497768721$ Hartree

Table 15. Standard orientation of 6.3 THF [B3LYP/TZVP]

\begin{tabular}{ccccc} 
Center & Atom & \multicolumn{3}{c}{ Coordinates (Angstroms) } \\
Number & Type & $\mathrm{X}$ & $\mathrm{Y}$ & $\mathrm{Z}$ \\
\hline 1 & $\mathrm{H}$ & 0.137381 & 5.661974 & -1.373385 \\
2 & $\mathrm{H}$ & 1.571057 & 5.305249 & 0.626518 \\
3 & $\mathrm{C}$ & 0.516452 & 4.673938 & -1.139942
\end{tabular}




\begin{tabular}{|c|c|c|c|c|}
\hline 4 & C & 1.323929 & 4.472064 & -0.021664 \\
\hline 5 & $\mathrm{H}$ & -0.392468 & 3.733462 & -2.845587 \\
\hline 6 & $\mathrm{H}$ & -3.461210 & 4.944556 & 1.536137 \\
\hline 7 & C & 0.218292 & 3.590734 & -1.960716 \\
\hline 8 & $\mathrm{H}$ & -1.107422 & 4.338836 & 1.770629 \\
\hline 9 & $\mathrm{H}$ & 6.293822 & 2.149475 & -1.230358 \\
\hline 10 & C & 1.816034 & 3.205747 & 0.274356 \\
\hline 11 & C & -3.237230 & 3.924782 & 1.847696 \\
\hline 12 & $\mathrm{H}$ & 3.877597 & 2.437028 & -1.302986 \\
\hline 13 & $\mathrm{H}$ & -3.966792 & 3.631781 & 2.608209 \\
\hline 14 & $\mathrm{H}$ & 2.447129 & 3.057120 & 1.141993 \\
\hline 15 & C & -1.805202 & 3.755941 & 2.374167 \\
\hline 16 & $\mathrm{H}$ & -1.693586 & 4.058354 & 3.415860 \\
\hline 17 & C & 0.721739 & 2.325220 & -1.672376 \\
\hline 18 & $\mathrm{H}$ & -2.853570 & 3.398824 & -0.229579 \\
\hline 19 & C & 5.662781 & 1.413541 & -0.744185 \\
\hline 20 & C & 4.284154 & 1.581049 & -0.782518 \\
\hline 21 & C & 1.523290 & 2.105527 & -0.545722 \\
\hline 22 & C & -3.258375 & 2.942919 & 0.680105 \\
\hline 23 & $\mathrm{H}$ & 0.512300 & 1.492415 & -2.330802 \\
\hline 24 & $\mathrm{H}$ & -4.434240 & 1.035808 & -4.231512 \\
\hline 25 & $\mathrm{H}$ & -0.519934 & 2.030767 & 1.912659 \\
\hline 26 & $\mathrm{H}$ & -4.248405 & 2.536851 & 0.466521 \\
\hline 27 & $\mathrm{H}$ & 7.308521 & 0.187629 & -0.079133 \\
\hline 28 & $\mathrm{H}$ & -1.7 & 6045 & -3.549855 \\
\hline 29 & C & -1.549316 & 2.262913 & 2.179497 \\
\hline 30 & $\mathrm{H}$ & -4.438933 & 1.587930 & -1.937111 \\
\hline 31 & C & 6.23 & 6516 & -0.107413 \\
\hline 32 & $\mathrm{O}$ & -2.404088 & 1.849283 & 1.077394 \\
\hline 33 & $\mathrm{H}$ & -2.948670 & -0.730105 & -4.976800 \\
\hline 34 & C & 3.414444 & 0.654124 & -0.171404 \\
\hline 35 & C & -4.426564 & 0.146554 & -3.597092 \\
\hline 36 & $\mathrm{~N}$ & 2.009863 & 0.805932 & -0.233237 \\
\hline 37 & $\mathrm{H}$ & -1.828634 & 1.678953 & 3.060520 \\
\hline 38 & C & 3433 & 0.538194 & -2.126966 \\
\hline 39 & C & -2.122017 & -0.070246 & -3.065308 \\
\hline 40 & C & -3.201742 & -0.723081 & -3.916626 \\
\hline 41 & $\mathrm{O}$ & -2.802433 & 0.309636 & -1.846406 \\
\hline 42 & $\mathrm{H}$ & -5.37 & -0.376252 & -3.743594 \\
\hline 43 & C & 5.386050 & -0.615716 & 0.484190 \\
\hline 44 & $\mathrm{H}$ & 2.753919 & -0.791493 & -2.287136 \\
\hline 45 & $\mathrm{H}$ & 1.38 & & 2.647645 \\
\hline 46 & $\mathrm{H}$ & -1.297115 & -0.726208 & -2.791091 \\
\hline 47 & C & 4.006690 & -0.457022 & 0.454407 \\
\hline 48 & $\mathrm{H}$ & -4.782544 & -0.078941 & -1.436276 \\
\hline 49 & $\mathrm{Li}$ & -1.9 & & -0.029530 \\
\hline 50 & $\mathrm{H}$ & 0486 & -1.755393 & -3.595864 \\
\hline 51 & $\mathrm{H}$ & 5.800191 & -1.485747 & 0.983465 \\
\hline 52 & Si & & -0.507910 & -0.000515 \\
\hline 53 & $\mathrm{H}$ & 3.16 & -2.479235 & -4.012049 \\
\hline 54 & C & 2.164804 & -1.700062 & -2.276646 \\
\hline 55 & $\mathrm{H}$ & 3.380254 & -1.200284 & 0.922722 \\
\hline 56 & C & & -0.365684 & 2.842556 \\
\hline 57 & $\mathrm{H}$ & 1.584337 & -0.102360 & 4.954923 \\
\hline 58 & C & 2.400444 & -2.658678 & -3.260034 \\
\hline 59 & C & 1.200930 & -1.887782 & -1.272186 \\
\hline 60 & C & 1.410329 & -0.813835 & 4.154261 \\
\hline 61 & C & 1.089750 & -1.245788 & 1.764551 \\
\hline 62 & O & -3.070659 & -1.213129 & 0.869211 \\
\hline 63 & $\mathrm{H}$ & -3.532425 & -0.438941 & 2.723981 \\
\hline 64 & $\mathrm{H}$ & -2.055787 & -1.432315 & 2.669724 \\
\hline 65 & C & -3.086227 & -1.344976 & 2.313973 \\
\hline
\end{tabular}




\begin{tabular}{ccccc}
66 & $\mathrm{H}$ & -4.424124 & -2.333488 & -0.227576 \\
67 & $\mathrm{C}$ & 1.678445 & -3.848528 & -3.273291 \\
68 & $\mathrm{C}$ & 0.469148 & -3.087951 & -1.329361 \\
69 & $\mathrm{C}$ & -3.451571 & -2.467566 & 0.256311 \\
70 & $\mathrm{H}$ & -2.711924 & -2.720947 & -0.505129 \\
71 & $\mathrm{C}$ & 1.314277 & -2.173846 & 4.442081 \\
72 & $\mathrm{H}$ & 1.868339 & -4.599649 & -4.031362 \\
73 & $\mathrm{C}$ & 1.011978 & -2.611070 & 2.083821 \\
74 & $\mathrm{H}$ & -0.313831 & -3.273519 & -0.599217 \\
75 & $\mathrm{C}$ & 0.705665 & -4.058773 & -2.299295 \\
76 & $\mathrm{H}$ & 1.405326 & -2.528306 & 5.462335 \\
77 & $\mathrm{H}$ & 0.891639 & -3.342703 & 1.293235 \\
78 & $\mathrm{C}$ & 1.118618 & -3.070340 & 3.396049 \\
79 & $\mathrm{C}$ & -3.886663 & -2.611344 & 2.597378 \\
80 & $\mathrm{H}$ & -4.958567 & -2.396034 & 2.610768 \\
81 & $\mathrm{C}$ & -3.527335 & -3.488968 & 1.391186 \\
82 & $\mathrm{H}$ & 0.131486 & -4.979629 & -2.295825 \\
83 & $\mathrm{H}$ & -3.616400 & -3.065207 & 3.550803 \\
84 & $\mathrm{H}$ & -4.260905 & -4.271199 & 1.193043 \\
85 & $\mathrm{H}$ & 1.065254 & -4.135299 & 3.598797 \\
86 & $\mathrm{H}$ & -2.555564 & -3.962360 & 1.545580 \\
\hline
\end{tabular}

Total Energy of 6.3 THF [B3LYP/TZVP]: E(SCF) $=-1975.2595826839$

Table 16. Standard orientation of 4 [B3LYP/6-31+G(d)]

\begin{tabular}{ccccc}
$\begin{array}{c}\text { Center } \\
\text { Number }\end{array}$ & $\begin{array}{c}\text { Atom } \\
\text { Type }\end{array}$ & \multicolumn{3}{c}{ Coordinates (Angstroms) } \\
\hline 1 & $\mathrm{C}$ & -11.526421 & 6.758750 & -1.175812 \\
2 & $\mathrm{C}$ & -10.177654 & 6.397603 & -1.372064 \\
3 & $\mathrm{C}$ & -9.548574 & 6.914565 & -2.520798 \\
4 & $\mathrm{C}$ & -10.218579 & 7.737567 & -3.432540 \\
5 & $\mathrm{C}$ & -11.556947 & 8.073264 & -3.212468 \\
6 & $\mathrm{C}$ & -12.209351 & 7.580499 & -2.077904 \\
7 & $\mathrm{Si}$ & -9.215235 & 5.194936 & -0.200629 \\
8 & $\mathrm{~N}$ & -9.731321 & 3.516046 & -0.529787 \\
9 & $\mathrm{C}$ & -11.162399 & 3.185660 & -0.529144 \\
10 & $\mathrm{C}$ & -11.492920 & 1.766889 & -0.043154 \\
11 & $\mathrm{C}$ & -9.900922 & 5.587614 & 1.551938 \\
12 & $\mathrm{C}$ & -10.147566 & 6.911788 & 1.969948 \\
13 & $\mathrm{C}$ & -10.518101 & 7.213768 & 3.284095 \\
14 & $\mathrm{C}$ & -10.655234 & 6.190685 & 4.227670 \\
15 & $\mathrm{C}$ & -10.416180 & 4.868334 & 3.840984 \\
16 & $\mathrm{C}$ & -10.041611 & 4.577764 & 2.525176 \\
17 & $\mathrm{C}$ & -8.888419 & 2.628978 & -1.328510 \\
18 & $\mathrm{C}$ & -9.149327 & 2.633973 & -2.845134 \\
19 & $\mathrm{Li}$ & -6.745320 & 5.509336 & -0.141865 \\
20 & $\mathrm{H}$ & -8.974981 & 1.595785 & -0.957597 \\
21 & $\mathrm{H}$ & -7.844022 & 2.923813 & -1.152308 \\
22 & $\mathrm{H}$ & -11.613623 & 3.334763 & -1.526049 \\
23 & $\mathrm{H}$ & -11.663855 & 3.896709 & 0.136016 \\
24 & $\mathrm{H}$ & -9.857075 & 3.543295 & 2.241392 \\
25 & $\mathrm{H}$ & -10.523896 & 4.062362 & 4.564555 \\
26 & $\mathrm{H}$ & -10.947384 & 6.421074 & 5.249715 \\
27 & $\mathrm{H}$ & -10.058511 & 7.726794 & 1.253063 \\
28 & $\mathrm{H}$ & -10.707565 & 8.246792 & 3.569617 \\
29 & $\mathrm{H}$ & -12.580847 & 1.648455 & 0.039191 \\
30 & $\mathrm{H}$ & -11.051953 & 1.577217 & 0.942924 \\
31 & $\mathrm{H}$ & -11.132259 & 0.993061 & -0.730809 \\
32 & $\mathrm{H}$ & -8.448717 & 1.959274 & -3.356082
\end{tabular}




\begin{tabular}{llcll}
33 & $\mathrm{H}$ & -9.027320 & 3.641433 & -3.260614 \\
34 & $\mathrm{H}$ & -10.164693 & 2.296980 & -3.084341 \\
35 & $\mathrm{H}$ & -8.503878 & 6.665385 & -2.713029 \\
36 & $\mathrm{H}$ & -9.697676 & 8.117167 & -4.309506 \\
37 & $\mathrm{H}$ & -12.085528 & 8.713746 & -3.914912 \\
38 & $\mathrm{H}$ & -13.250790 & 7.837877 & -1.894811 \\
39 & $\mathrm{H}$ & -12.056237 & 6.398795 & -0.296189 \\
\hline
\end{tabular}

Total Energy of 4 [B3LYP/6-31+G(d)]: E(RB+HF-LYP) $=-973.555479420$ Hartree Corrected Zero Point Energy: -973.232975 Hartree

Table 17. Standard orientation of $5[B 3 L Y P / 6-31+G(d)]$

\begin{tabular}{ccccc}
$\begin{array}{c}\text { Center } \\
\text { Number }\end{array}$ & Atom & \multicolumn{3}{c}{ Coordinates (Angstroms) } \\
\hline 1 & $\mathrm{C}$ & $\mathrm{X}$ & $\mathrm{Y}$ & $\mathrm{Z}$ \\
\hline 2 & $\mathrm{C}$ & 1.808260 & 4.171905 & 1.994596 \\
3 & $\mathrm{C}$ & 0.846260 & 5.150746 & 2.727100 \\
4 & $\mathrm{C}$ & 1.252934 & 6.639323 & 2.147620 \\
5 & $\mathrm{C}$ & 1.932667 & 5.653098 & 0.111176 \\
6 & $\mathrm{C}$ & 2.224790 & 4.389748 & 0.665903 \\
7 & $\mathrm{Si}$ & 3.341281 & 3.122800 & -0.259816 \\
8 & $\mathrm{~N}$ & 2.818670 & 3.299829 & -1.964143 \\
9 & $\mathrm{C}$ & 3.751835 & 2.963609 & -3.037927 \\
10 & $\mathrm{C}$ & 3.668695 & 1.526327 & -3.582428 \\
11 & $\mathrm{~N}$ & 2.923558 & 1.548284 & 0.478455 \\
12 & $\mathrm{C}$ & 1.547551 & 1.053409 & 0.575731 \\
13 & $\mathrm{H}$ & 1.346039 & 0.679954 & 1.594095 \\
14 & $\mathrm{C}$ & 3.946394 & 0.605709 & 0.916252 \\
15 & $\mathrm{C}$ & 4.067178 & 0.448184 & 2.442292 \\
16 & $\mathrm{C}$ & 1.407021 & 3.319606 & -2.362247 \\
17 & $\mathrm{C}$ & 1.057325 & 4.337121 & -3.460081 \\
18 & $\mathrm{Li}$ & 5.711741 & 3.792262 & 0.068314 \\
19 & $\mathrm{H}$ & 3.631883 & 3.666924 & -3.876923 \\
20 & $\mathrm{H}$ & 4.769162 & 3.127639 & -2.653094 \\
21 & $\mathrm{H}$ & 1.067607 & 2.322318 & -2.694081 \\
22 & $\mathrm{H}$ & 0.813304 & 3.561065 & -1.474839 \\
23 & $\mathrm{H}$ & 2.241704 & 5.864896 & -0.910780 \\
24 & $\mathrm{H}$ & 1.037701 & 7.600180 & 0.368455 \\
25 & $\mathrm{H}$ & 0.868831 & 1.902076 & 0.435896 \\
26 & $\mathrm{C}$ & 1.171109 & -0.050298 & -0.428790 \\
27 & $\mathrm{H}$ & 3.782326 & -0.389648 & 0.470927 \\
28 & $\mathrm{H}$ & 4.914432 & 0.949264 & 0.524249 \\
29 & $\mathrm{H}$ & 0.315488 & 7.154634 & 2.712421 \\
30 & $\mathrm{H}$ & 2.019598 & 3.212570 & 2.462627 \\
31 & $\mathrm{H}$ & 0.815208 & 4.943701 & 3.748794 \\
32 & $\mathrm{H}$ & -0.029583 & 4.359751 & -3.611824 \\
33 & $\mathrm{H}$ & 1.384578 & 5.345133 & -3.179051 \\
34 & $\mathrm{H}$ & 1.515532 & 4.088496 & -4.424450 \\
35 & $\mathrm{H}$ & 4.423985 & 1.370432 & -4.365043 \\
36 & $\mathrm{H}$ & 3.841384 & 0.795681 & -2.783686 \\
37 & $\mathrm{H}$ & 2.688734 & 1.313199 & -4.025739 \\
38 & $\mathrm{H}$ & 3.131066 & 0.095715 & 2.891029 \\
39 & $\mathrm{H}$ & 4.849419 & -0.280911 & 2.696105 \\
40 & $\mathrm{H}$ & 4.320404 & 1.407232 & 2.911554 \\
41 & $\mathrm{H}$ & 0.118722 & -0.336634 & -0.298400 \\
42 & $\mathrm{H}$ & 1.307732 & 0.293222 & -1.459854 \\
43 & $\mathrm{H}$ & 1.775836 & -0.954669 & -0.291617 \\
\hline & & & & \\
\hline & & & & \\
13 & &
\end{tabular}

Total Energy of 5 [B3LYP/6-31+G(d)]: E(RB+HF-LYP) $=-955.114535419$ Hartree 
Table 18. Standard orientation of $6[B 3 L Y P / 6-31+G(d)]$

\begin{tabular}{|c|c|c|c|c|}
\hline \multirow{2}{*}{$\begin{array}{l}\text { Center } \\
\text { Number }\end{array}$} & \multirow{2}{*}{$\begin{array}{l}\text { Atom } \\
\text { Type }\end{array}$} & \multicolumn{3}{|c|}{ Coordinates (Angstroms) } \\
\hline & & X & $\mathrm{Y}$ & Z \\
\hline 1 & C & 0.291754 & -3.058599 & -1.356082 \\
\hline 2 & C & 1.118622 & -1.915566 & -1.331620 \\
\hline 3 & C & 2.123632 & -1.834146 & -2.314592 \\
\hline 4 & C & 2.303116 & -2.844812 & -3.265690 \\
\hline 5 & C & 1.477091 & -3.971664 & -3.260458 \\
\hline 6 & C & 0.466907 & -4.075519 & -2.298197 \\
\hline 7 & $\mathrm{Si}$ & 0.729413 & -0.502716 & -0.098800 \\
\hline 8 & C & 1.083093 & -1.202566 & 1.659826 \\
\hline 9 & C & 1.438921 & -2.539358 & 1.922084 \\
\hline 10 & C & 1.607732 & -3.011504 & 3.228763 \\
\hline 11 & C & 1.434478 & -2.150906 & 4.315594 \\
\hline 12 & C & 1.081680 & -0.817207 & 4.083668 \\
\hline 13 & C & 0.899214 & -0.361519 & 2.775946 \\
\hline 14 & $\mathrm{~N}$ & 2.015510 & 0.815528 & -0.442635 \\
\hline 15 & C & 1.432916 & 2.073890 & -0.730074 \\
\hline 16 & C & 1.621827 & 3.192695 & 0.108425 \\
\hline 17 & C & 0.915016 & 4.376652 & -0.103662 \\
\hline 18 & C & 0.000404 & 4.491723 & -1.160182 \\
\hline 19 & C & -0.167087 & 3.410515 & -2.031334 \\
\hline 20 & C & 0.553879 & 2.221513 & -1.831152 \\
\hline 21 & C & 3.426806 & 0.762998 & -0.316864 \\
\hline 22 & C & 4.059709 & -0.263142 & 0.411471 \\
\hline 23 & C & 5.450808 & -0.330968 & 0.491442 \\
\hline 24 & C & 6.257854 & 0.618054 & -0.141920 \\
\hline 25 & C & 5.641770 & 1.636463 & -0.873949 \\
\hline 26 & C & 4.251408 & 1.706460 & -0.969274 \\
\hline 27 & $\mathrm{Li}$ & -1.042522 & 1.187833 & -0.548624 \\
\hline 28 & $\mathrm{H}$ & 3.462166 & -1.006308 & 0.923427 \\
\hline 29 & $\mathrm{H}$ & 5.904088 & -1.136339 & 1.064896 \\
\hline 30 & $\mathrm{H}$ & 7.340791 & 0.561976 & -0.071830 \\
\hline 31 & $\mathrm{H}$ & 6.244919 & 2.380476 & -1.389880 \\
\hline 32 & $\mathrm{H}$ & 3.802050 & 2.493672 & -1.567161 \\
\hline 33 & $\mathrm{H}$ & 2.307945 & 3.106741 & 0.946051 \\
\hline 34 & $\mathrm{H}$ & 1.063582 & 5.213217 & 0.574962 \\
\hline 35 & $\mathrm{H}$ & -0.552286 & 5.414348 & -1.313147 \\
\hline 36 & $\mathrm{H}$ & -0.827760 & 3.498476 & -2.891560 \\
\hline 37 & $\mathrm{H}$ & 0.478988 & 1.404956 & -2.547044 \\
\hline 38 & $\mathrm{H}$ & 1.599019 & -3.225474 & 1.093560 \\
\hline 39 & $\mathrm{H}$ & 1.882369 & -4.051149 & 3.395795 \\
\hline 40 & $\mathrm{H}$ & 1.569700 & -2.514334 & 5.331646 \\
\hline 41 & $\mathrm{H}$ & 0.942794 & -0.136628 & 4.921335 \\
\hline 42 & $\mathrm{H}$ & 0.608151 & 0.677713 & 2.621273 \\
\hline 43 & $\mathrm{H}$ & 2.784116 & -0.971322 & -2.336390 \\
\hline 44 & $\mathrm{H}$ & 3.093639 & -2.752660 & -4.007774 \\
\hline 45 & $\mathrm{H}$ & 1.616569 & -4.759383 & -3.997260 \\
\hline 46 & $\mathrm{H}$ & -0.185667 & -4.946224 & -2.284019 \\
\hline 47 & $\mathrm{H}$ & -0.512253 & -3.157769 & -0.626762 \\
\hline
\end{tabular}

Total Energy of 6 [B3LYP/6-31+G(d)]: E(RB+HF-LYP) $=-1278.43395930$ Hartree Corrected Zero Point Energy: -1278.062874 Hartree

Table 19. Standard orientation of $\mathrm{Me}_{3} \mathrm{SiH}[\mathrm{B} 3 \mathrm{LYP} / 6-31+\mathrm{G}(\mathrm{d})]$ 


\begin{tabular}{ccccc}
$\begin{array}{c}\text { Center } \\
\text { Number }\end{array}$ & $\begin{array}{c}\text { Atom } \\
\text { Type }\end{array}$ & \multicolumn{3}{c}{ Coordinates (Angstroms) } \\
\hline 1 & $\mathrm{C}$ & -1.717422 & -0.524898 & -0.224118 \\
2 & $\mathrm{Si}$ & -0.000453 & -0.000649 & 0.379150 \\
3 & $\mathrm{C}$ & 1.314429 & -1.222747 & -0.223728 \\
4 & $\mathrm{H}$ & -0.001347 & -0.001279 & 1.874123 \\
5 & $\mathrm{C}$ & 0.403686 & 1.748382 & -0.223277 \\
6 & $\mathrm{H}$ & -1.763286 & -0.556921 & -1.320289 \\
7 & $\mathrm{H}$ & -2.489522 & 0.175057 & 0.119597 \\
8 & $\mathrm{H}$ & -1.984479 & -1.521687 & 0.149055 \\
9 & $\mathrm{H}$ & 1.386110 & 2.077244 & 0.138563 \\
10 & $\mathrm{H}$ & -0.339884 & 2.474322 & 0.129307 \\
11 & $\mathrm{H}$ & 0.420697 & 1.795981 & -1.319533 \\
12 & $\mathrm{H}$ & 1.341205 & -1.269110 & -1.319834 \\
13 & $\mathrm{H}$ & 1.117517 & -2.236549 & 0.147163 \\
14 & $\mathrm{H}$ & 2.315178 & -0.932388 & 0.120489 \\
\hline
\end{tabular}

Total Energy of $\mathrm{Me}_{3} \mathrm{SiH}[\mathrm{B} 3 \mathrm{LYP} / 6-31+\mathrm{G}(\mathrm{d})]$ : $\mathrm{E}(\mathrm{RB}+\mathrm{HF}-\mathrm{LYP})=-409.871446420$ Hartree Corrected Zero Point Energy: -409.752065 Hartree

Table 20. Standard orientation of $\mathrm{Me}_{3} \mathrm{SiCl}[\mathrm{B} 3 \mathrm{LYP} / 6-31+\mathrm{G}(\mathrm{d})]$

\begin{tabular}{ccccc}
$\begin{array}{c}\text { Center } \\
\text { Number }\end{array}$ & Atom & \multicolumn{3}{c}{ Coordinates (Angstroms) } \\
\hline 1 & $\mathrm{Si}$ & -0.350999 & 0.000214 & -0.000118 \\
2 & $\mathrm{Cl}$ & 1.764065 & 0.000611 & 0.000402 \\
3 & $\mathrm{C}$ & -0.891590 & 1.750204 & -0.429297 \\
4 & $\mathrm{C}$ & -0.889517 & -1.247467 & -1.301369 \\
5 & $\mathrm{C}$ & -0.891018 & -0.504047 & 1.730110 \\
6 & $\mathrm{H}$ & -1.986906 & 1.827475 & -0.452219 \\
7 & $\mathrm{H}$ & -0.512249 & 2.050150 & -1.413005 \\
8 & $\mathrm{H}$ & -0.518529 & 2.470214 & 0.308357 \\
9 & $\mathrm{H}$ & -0.512924 & -2.249667 & -1.065905 \\
10 & $\mathrm{H}$ & -0.511578 & -0.971220 & -2.292592 \\
11 & $\mathrm{H}$ & -1.984764 & -1.303850 & -1.361492 \\
12 & $\mathrm{H}$ & -1.986390 & -0.530244 & 1.806960 \\
13 & $\mathrm{H}$ & -0.517373 & 0.201543 & 2.481226 \\
14 & $\mathrm{H}$ & -0.511654 & -1.499929 & 1.986819 \\
\hline
\end{tabular}

Total Energy of $\mathrm{Me}_{3} \mathrm{SiCl}[\mathrm{B} 3 \mathrm{LYP} / 6-31+\mathrm{G}(\mathrm{d})]$ : $\mathrm{E}(\mathrm{RB}+\mathrm{HF}-\mathrm{LYP})=-869.529324274$ Hartree Corrected Zero Point Energy: -869.416168 Hartree

Table 21. Standard orientation of Me3SiLi (2) [B3LYP/6-31+G(d)]

\begin{tabular}{ccccc} 
Center & Atom & \multicolumn{3}{c}{ Coordinates (Angstroms) } \\
Number & Type & $\mathrm{X}$ & $\mathrm{Y}$ & $\mathrm{Z}$ \\
\hline 1 & $\mathrm{Si}$ & -0.000007 & -0.000474 & 0.344877 \\
2 & $\mathrm{Li}$ & -0.004215 & -0.003259 & 2.862381 \\
3 & $\mathrm{C}$ & -1.263310 & -1.223610 & -0.443852 \\
4 & $\mathrm{C}$ & -0.428278 & 1.706558 & -0.440718 \\
5 & $\mathrm{C}$ & 1.692845 & -0.481413 & -0.440634 \\
6 & $\mathrm{H}$ & -1.224347 & -1.182609 & -1.543445 \\
7 & $\mathrm{H}$ & -2.293164 & -0.990288 & -0.141163 \\
8 & $\mathrm{H}$ & -1.062408 & -2.261258 & -0.144666 \\
9 & $\mathrm{H}$ & 0.303507 & 2.475384 & -0.157665 \\
10 & $\mathrm{H}$ & -1.416521 & 2.063068 & -0.120115 \\
11 & $\mathrm{H}$ & -0.438175 & 1.648063 & -1.540195 \\
12 & $\mathrm{H}$ & 1.644097 & -0.455838 & -1.540304 \\
13 & $\mathrm{H}$ & 2.000343 & -1.494355 & -0.147329
\end{tabular}


Total Energy of $\mathrm{Me}_{3} \mathrm{SiLi}(2)$ [B3LYP/6-31+G(d)]: E(RB+HF-LYP) = -416.770643918 Hartree Corrected Zero Point Energy: -416.659456 Hartree

Table 22. Standard orientation of $\mathrm{Me}_{4} \mathrm{Si}[\mathrm{B} 3 \mathrm{LYP} / 6-31+\mathrm{G}(\mathrm{d})]$

\begin{tabular}{ccccc}
$\begin{array}{c}\text { Center } \\
\text { Number }\end{array}$ & Atom & \multicolumn{3}{c}{ Coordinates (Angstroms) } \\
\hline 1 & $\mathrm{Si}$ & 0.000215 & -0.000169 & 0.000842 \\
2 & $\mathrm{C}$ & 1.123432 & -1.344569 & 0.729441 \\
3 & $\mathrm{C}$ & 0.416991 & 0.247084 & -1.833529 \\
4 & $\mathrm{C}$ & -1.813604 & -0.531382 & 0.168884 \\
5 & $\mathrm{C}$ & 0.272875 & 1.629082 & 0.934300 \\
6 & $\mathrm{H}$ & 0.953868 & -1.465384 & 1.807055 \\
7 & $\mathrm{H}$ & 2.184595 & -1.100941 & 0.587742 \\
8 & $\mathrm{H}$ & 0.941631 & -2.318058 & 0.255177 \\
9 & $\mathrm{H}$ & -0.186992 & 1.050233 & -2.275909 \\
10 & $\mathrm{H}$ & 0.228760 & -0.665334 & -2.414658 \\
11 & $\mathrm{H}$ & 1.472849 & 0.513724 & -1.970706 \\
12 & $\mathrm{H}$ & -1.991180 & -1.501761 & -0.312356 \\
13 & $\mathrm{H}$ & -2.488882 & 0.197468 & -0.298056 \\
14 & $\mathrm{H}$ & -2.108523 & -0.626559 & 1.222329 \\
15 & $\mathrm{H}$ & -0.332231 & 2.440323 & 0.508685 \\
16 & $\mathrm{H}$ & 1.323910 & 1.943390 & 0.890598 \\
17 & $\mathrm{H}$ & 0.001013 & 1.533977 & 1.993731 \\
\hline
\end{tabular}

Total Energy of $\mathrm{Me}_{4} \mathrm{Si}[\mathrm{B} 3 \mathrm{LYP} / 6-31+\mathrm{G}(\mathrm{d})]$ : $E(\mathrm{RB}+\mathrm{HF}-\mathrm{LYP})=-449.199687169$ Hartree Corrected Zero Point Energy: -449.051860 Hartree

Table 23. Standard orientation of $(\mathrm{MeO}) \mathrm{Me}_{2} \mathrm{SiH}[\mathrm{B} 3 \mathrm{LYP} / 6-31+\mathrm{G}(\mathrm{d})]$

\begin{tabular}{ccccc}
$\begin{array}{c}\text { Center } \\
\text { Number }\end{array}$ & Atom & \multicolumn{3}{c}{ Coordinates (Angstroms) } \\
\hline 1 & $\mathrm{Si}$ & -0.411388 & -0.000481 & 0.349525 \\
2 & $\mathrm{C}$ & -1.801134 & -1.140071 & -0.189393 \\
3 & $\mathrm{O}$ & 0.989471 & -0.629958 & -0.322620 \\
4 & $\mathrm{C}$ & -0.702905 & 1.780054 & -0.203479 \\
5 & $\mathrm{C}$ & 2.293796 & -0.161797 & -0.017318 \\
6 & $\mathrm{H}$ & -0.263788 & -0.010803 & 1.838647 \\
7 & $\mathrm{H}$ & -2.762443 & -0.815825 & 0.228974 \\
8 & $\mathrm{H}$ & -1.615639 & -2.167598 & 0.144202 \\
9 & $\mathrm{H}$ & -1.895123 & -1.153518 & -1.282072 \\
10 & $\mathrm{H}$ & -0.819661 & 1.844989 & -1.292622 \\
11 & $\mathrm{H}$ & 0.125955 & 2.438307 & 0.085568 \\
12 & $\mathrm{H}$ & -1.614891 & 2.183442 & 0.256343 \\
13 & $\mathrm{H}$ & 3.014491 & -0.840669 & -0.484084 \\
14 & $\mathrm{H}$ & 2.475154 & -0.150913 & 1.067557 \\
15 & $\mathrm{H}$ & 2.461079 & 0.849867 & -0.413756 \\
\hline
\end{tabular}

Total Energy of $(\mathrm{MeO}) \mathrm{Me}_{2} \mathrm{SiH}$ [B3LYP/6-31+G(d)]: E(RB+HF-LYP $)=-485.117828328$ Hartree Corrected Zero Point Energy: -484.992709 Hartree

Table 24. Standard orientation of $(\mathrm{MeO}) \mathrm{Me}_{2} \mathrm{SiCl}[\mathrm{B} 3 \mathrm{LYP} / 6-31+\mathrm{G}(\mathrm{d})]$

\begin{tabular}{ccccc} 
Center & Atom & \multicolumn{3}{c}{ Coordinates(Angstroms) } \\
Number & Type & $\mathrm{X}$ & $\mathrm{Y}$ & $\mathrm{Z}$ \\
\hline 1 & Si & -0.220567 & -0.385023 & 0.009483
\end{tabular}




\begin{tabular}{ccccc}
2 & $\mathrm{C}$ & -0.225621 & -0.931083 & 1.804784 \\
3 & $\mathrm{Cl}$ & -0.927695 & 1.602197 & -0.013668 \\
4 & $\mathrm{O}$ & 1.302117 & -0.405210 & -0.645746 \\
5 & $\mathrm{C}$ & -1.297130 & -1.416395 & -1.113612 \\
6 & $\mathrm{C}$ & 2.433542 & 0.328550 & -0.188688 \\
7 & $\mathrm{H}$ & -1.242282 & -0.910154 & 2.214786 \\
8 & $\mathrm{H}$ & 0.395100 & -0.277466 & 2.428341 \\
9 & $\mathrm{H}$ & 0.158117 & -1.955265 & 1.894997 \\
10 & $\mathrm{H}$ & -0.946938 & -2.455794 & -1.130377 \\
11 & $\mathrm{H}$ & -1.263536 & -1.029638 & -2.137921 \\
12 & $\mathrm{H}$ & -2.340462 & -1.409456 & -0.778064 \\
13 & $\mathrm{H}$ & 3.270671 & 0.090564 & -0.850795 \\
14 & $\mathrm{H}$ & 2.704006 & 0.047476 & 0.837849 \\
15 & $\mathrm{H}$ & 2.242379 & 1.407962 & -0.228166 \\
\hline
\end{tabular}

Total Energy of $(\mathrm{MeO}) \mathrm{Me}_{2} \mathrm{SiCl}$ [B3LYP/6-31+G(d)]: $\mathrm{E}(\mathrm{RB}+\mathrm{HF}-\mathrm{LYP})=-944.777827535$ Hartree Corrected Zero Point Energy: -944.658755 Hartree

Table 25. Standard orientation of (MeO)Me ${ }_{2}$ SiLi [B3LYP/6-31+G(d)]

\begin{tabular}{|c|c|c|c|c|}
\hline \multirow{2}{*}{$\begin{array}{l}\text { Center } \\
\text { Number }\end{array}$} & \multirow{2}{*}{$\begin{array}{l}\text { Atom } \\
\text { Type }\end{array}$} & \multicolumn{3}{|c|}{ Coordinates(Angstroms) } \\
\hline & & $\mathrm{x}$ & $\mathrm{Y}$ & Z \\
\hline 1 & $\mathrm{Si}$ & 0.407947 & -0.028849 & 0.343394 \\
\hline 2 & C & 0.731442 & 1.761745 & -0.276015 \\
\hline 3 & $\mathrm{Li}$ & 0.148836 & -0.309867 & 2.840615 \\
\hline 4 & $\mathrm{O}$ & -0.994874 & -0.576004 & -0.493249 \\
\hline 5 & C & 1.758539 & -1.083747 & -0.499190 \\
\hline 6 & C & -2.286273 & -0.109779 & -0.192713 \\
\hline 7 & $\mathrm{H}$ & 1.681344 & 2.141326 & 0.127342 \\
\hline 8 & $\mathrm{H}$ & -0.054480 & 2.461990 & 0.036611 \\
\hline 9 & $\mathrm{H}$ & 0.794159 & 1.793909 & -1.373798 \\
\hline 10 & $\mathrm{H}$ & 1.736367 & -0.944084 & -1.589295 \\
\hline 11 & $\mathrm{H}$ & 1.620263 & -2.152896 & -0.295747 \\
\hline 12 & $\mathrm{H}$ & 2.757956 & -0.800609 & -0.140754 \\
\hline 13 & $\mathrm{H}$ & -3.015582 & -0.711236 & -0.749332 \\
\hline 14 & $\mathrm{H}$ & -2.423885 & 0.945871 & -0.476796 \\
\hline 15 & $\mathrm{H}$ & -2.517168 & -0.202065 & 0.885911 \\
\hline
\end{tabular}

Total Energy of (MeO)Me ${ }_{2} \mathrm{SiLi}[\mathrm{B} 3 \mathrm{LYP} / 6-31+\mathrm{G}(\mathrm{d})]$ : $\mathrm{E}(\mathrm{RB}+\mathrm{HF}-\mathrm{LYP})=-492.018232106$ Hartree Corrected Zero Point Energy: -491.901494 Hartree

Table 26. Standard orientation of $(\mathrm{MeO}) \mathrm{Me}_{2} \mathrm{SiMe}[\mathrm{B} 3 \mathrm{LYP} / 6-31+\mathrm{G}(\mathrm{d})]$

\begin{tabular}{ccccc}
$\begin{array}{c}\text { Center } \\
\text { Number }\end{array}$ & Atom & \multicolumn{3}{c}{ Coordinates(Angstroms) } \\
\hline 1 & $\mathrm{Si}$ & 0.381353 & 0.003443 & 0.004784 \\
2 & $\mathrm{C}$ & 0.571401 & 1.852381 & 0.358181 \\
3 & $\mathrm{O}$ & -1.080878 & -0.289995 & -0.772211 \\
4 & $\mathrm{C}$ & 1.688571 & -0.597066 & -1.206345 \\
5 & $\mathrm{C}$ & -2.361702 & -0.078346 & -0.202970 \\
6 & $\mathrm{H}$ & 0.472574 & -0.961451 & 1.629208 \\
7 & $\mathrm{H}$ & 1.543473 & 2.063195 & 0.823708 \\
8 & $\mathrm{H}$ & -0.202557 & 2.219623 & 1.044323 \\
9 & $\mathrm{H}$ & 0.508890 & 2.442308 & -0.564576 \\
10 & $\mathrm{H}$ & 1.593308 & -0.082966 & -2.170475 \\
11 & $\mathrm{H}$ & 1.582549 & -1.672698 & -1.391903 \\
12 & $\mathrm{H}$ & 2.701973 & -0.415858 & -0.826439 \\
13 & $\mathrm{H}$ & -3.113242 & -0.373314 & -0.942620 \\
14 & $\mathrm{H}$ & -2.523839 & 0.979381 & 0.051168
\end{tabular}




\begin{tabular}{ccccc}
15 & $\mathrm{H}$ & -2.509522 & -0.685061 & 0.701965 \\
16 & $\mathrm{H}$ & -0.308706 & -0.661941 & 2.339159 \\
17 & $\mathrm{H}$ & 1.439256 & -0.793822 & 2.122278 \\
18 & $\mathrm{H}$ & 0.371442 & -2.040205 & 1.455669 \\
\hline
\end{tabular}

Total Energy of $(\mathrm{MeO}) \mathrm{Me}_{2} \mathrm{SiMe}[\mathrm{B} 3 \mathrm{LYP} / 6-31+\mathrm{G}(\mathrm{d})]$ : $\mathrm{E}(\mathrm{RB}+\mathrm{HF}-\mathrm{LYP})=-524.447046721$ Hartree Corrected Zero Point Energy: -524.293493 Hartree

Table 27. Standard orientation of $\left(\mathrm{Me}_{2} \mathrm{~N}\right) \mathrm{Me}_{2} \mathrm{SiH}[\mathrm{B} 3 \mathrm{LYP} / 6-31+\mathrm{G}(\mathrm{d})]$

\begin{tabular}{ccccc}
$\begin{array}{c}\text { Center } \\
\text { Number }\end{array}$ & $\begin{array}{c}\text { Atom } \\
\text { Type }\end{array}$ & \multicolumn{3}{c}{ Coordinates(Angstroms) } \\
\hline 1 & $\mathrm{H}$ & 0.541239 & 0.000000 & -0.226088 \\
2 & $\mathrm{Si}$ & 0.100635 & 0.000000 & 1.211144 \\
3 & $\mathrm{~N}$ & 1.460562 & 0.000000 & 2.319584 \\
4 & $\mathrm{C}$ & 2.259673 & 1.206965 & 2.497346 \\
5 & $\mathrm{H}$ & 3.067048 & 1.300047 & 1.747292 \\
6 & $\mathrm{H}$ & 1.633263 & 2.101686 & 2.433854 \\
7 & $\mathrm{H}$ & 2.731680 & 1.204375 & 3.491082 \\
8 & $\mathrm{C}$ & -0.937595 & 1.553419 & 1.484304 \\
9 & $\mathrm{C}$ & -0.937595 & -1.553419 & 1.484304 \\
10 & $\mathrm{C}$ & 2.259673 & -1.206965 & 2.497346 \\
11 & $\mathrm{H}$ & 3.067048 & -1.300047 & 1.747292 \\
12 & $\mathrm{H}$ & 2.731680 & -1.204375 & 3.491082 \\
13 & $\mathrm{H}$ & 1.633263 & -2.101686 & 2.433854 \\
14 & $\mathrm{H}$ & -1.250215 & -1.647122 & 2.531655 \\
15 & $\mathrm{H}$ & -1.843011 & -1.503211 & 0.865641 \\
16 & $\mathrm{H}$ & -0.407189 & -2.470902 & 1.203959 \\
17 & $\mathrm{H}$ & -1.250215 & 1.647122 & 2.531655 \\
18 & $\mathrm{H}$ & -0.407189 & 2.470902 & 1.203959 \\
19 & $\mathrm{H}$ & -1.843011 & 1.503211 & 0.865641 \\
\hline
\end{tabular}

Total Energy of $\left(\mathrm{Me}_{2} \mathrm{~N}\right) \mathrm{Me}_{2} \mathrm{SiH}$ [B3LYP/6-31+G(d)]: $\mathrm{E}(\mathrm{RB}+\mathrm{HF}-\mathrm{LYP})=-504.539321387$ Hartree Corrected Zero Point Energy: -504.373829 Hartree

Table 28. Standard orientation of $\left(\mathrm{Me}_{2} \mathrm{~N}\right) \mathrm{Me}_{2} \mathrm{SiCl}[\mathrm{B} 3 \mathrm{LYP} / 6-31+\mathrm{G}(\mathrm{d})]$

\begin{tabular}{ccccc}
$\begin{array}{c}\text { Center } \\
\text { Number }\end{array}$ & Atom & \multicolumn{3}{c}{ Coordinates(Angstroms) } \\
\hline 1 & $\mathrm{Cl}$ & $\mathrm{X}$ & $\mathrm{Y}$ & $\mathrm{Z}$ \\
\hline 2 & $\mathrm{Si}$ & 0.011575 & -0.808219 & 0.504189 \\
3 & $\mathrm{~N}$ & 1.532276 & 0.020579 & 2.421558 \\
4 & $\mathrm{C}$ & 2.332234 & 1.507210 & 3.283503 \\
5 & $\mathrm{H}$ & 3.111614 & 1.336365 & 2.925576 \\
6 & $\mathrm{H}$ & 1.701179 & 2.368326 & 2.513024 \\
7 & $\mathrm{H}$ & 2.829923 & 1.977574 & 3.819952 \\
8 & $\mathrm{C}$ & -0.923766 & 1.570493 & 2.080064 \\
9 & $\mathrm{C}$ & -0.900849 & -1.299630 & 3.327752 \\
10 & $\mathrm{C}$ & 2.341969 & -0.638397 & 3.905138 \\
11 & $\mathrm{H}$ & 3.116123 & -1.026960 & 3.222540 \\
12 & $\mathrm{H}$ & 2.846729 & -0.239807 & 4.796624 \\
13 & $\mathrm{H}$ & 1.717370 & -1.476949 & 4.224729 \\
14 & $\mathrm{H}$ & -1.115543 & -0.992005 & 4.358545 \\
15 & $\mathrm{H}$ & -1.855495 & -1.462991 & 2.813879 \\
16 & $\mathrm{H}$ & -0.378460 & -2.261811 & 3.354388 \\
17 & $\mathrm{H}$ & -1.144197 & 2.108798 & 3.010080 \\
18 & $\mathrm{H}$ & -0.411806 & 2.255684 & 1.396082 \\
19 & $\mathrm{H}$ & -1.875352 & 1.294655 & 1.610513 \\
\hline
\end{tabular}


Total Energy of $\left(\mathrm{Me}_{2} \mathrm{~N}\right) \mathrm{Me}_{2} \mathrm{SiCl}[\mathrm{B} 3 \mathrm{LYP} / 6-31+\mathrm{G}(\mathrm{d})]$ : $\mathrm{E}(\mathrm{RB}+\mathrm{HF}-\mathrm{LYP})=-964.201459622$ Hartree Corrected Zero Point Energy: -964.041869 Hartree

Table 29. Standard orientation of $\left(\mathrm{Me}_{2} \mathrm{~N}\right) \mathrm{Me}_{2} \mathrm{SiLi}[\mathrm{B} 3 \mathrm{LYP} / 6-31+\mathrm{G}(\mathrm{d})]$

\begin{tabular}{ccccc}
$\begin{array}{c}\text { Center } \\
\text { Number }\end{array}$ & $\begin{array}{c}\text { Atom } \\
\text { Type }\end{array}$ & \multicolumn{3}{c}{ Coordinates(Angstroms) } \\
\hline 1 & Li & 1.099637 & 0.000000 & 0.246381 \\
2 & $\mathrm{Si}$ & 0.120094 & 0.000000 & 2.585325 \\
3 & $\mathrm{~N}$ & 1.414331 & 0.000000 & 3.847360 \\
4 & $\mathrm{C}$ & 2.234838 & 1.196295 & 3.943386 \\
5 & $\mathrm{H}$ & 2.961583 & 1.295949 & 3.106083 \\
6 & $\mathrm{H}$ & 1.610079 & 2.094967 & 3.952699 \\
7 & $\mathrm{H}$ & 2.818048 & 1.186716 & 4.876479 \\
8 & $\mathrm{C}$ & -0.978045 & 1.511356 & 3.026016 \\
9 & $\mathrm{C}$ & -0.978045 & -1.511356 & 3.026016 \\
10 & $\mathrm{C}$ & 2.234838 & -1.196295 & 3.943386 \\
11 & $\mathrm{H}$ & 2.961583 & -1.295949 & 3.106083 \\
12 & $\mathrm{H}$ & 2.818048 & -1.186716 & 4.876479 \\
13 & $\mathrm{H}$ & 1.610079 & -2.094967 & 3.952699 \\
14 & $\mathrm{H}$ & -1.261550 & -1.494254 & 4.088448 \\
15 & $\mathrm{H}$ & -1.900656 & -1.477906 & 2.430129 \\
16 & $\mathrm{H}$ & -0.496458 & -2.474511 & 2.817329 \\
17 & $\mathrm{H}$ & -1.261550 & 1.494254 & 4.088448 \\
18 & $\mathrm{H}$ & -0.496458 & 2.474511 & 2.817329 \\
19 & $\mathrm{H}$ & -1.900656 & 1.477906 & 2.430129 \\
\hline
\end{tabular}

Total Energy of $\left(\mathrm{Me}_{2} \mathrm{~N}\right) \mathrm{Me}_{2} \mathrm{SiLi}[\mathrm{B} 3 \mathrm{LYP} / 6-31+\mathrm{G}(\mathrm{d})]$ : $\mathrm{E}(\mathrm{RB}+\mathrm{HF}-\mathrm{LYP})=-511.439508474$ Hartree Corrected Zero Point Energy: -511.282287 Hartree

Table 30. Standard orientation of $\left(\mathrm{Me}_{2} \mathrm{~N}\right) \mathrm{Me}_{2} \mathrm{SiMe}[\mathrm{B} 3 \mathrm{LYP} / 6-31+\mathrm{G}(\mathrm{d})]$

\begin{tabular}{ccccc}
$\begin{array}{c}\text { Center } \\
\text { Number }\end{array}$ & $\begin{array}{c}\text { Atom } \\
\text { Type }\end{array}$ & \multicolumn{3}{c}{$\begin{array}{c}\text { Coordinates(Angstroms) } \\
\text { X }\end{array}$} \\
\hline 1 & $\mathrm{C}$ & 0.117911 & 0.032102 & 0.024453 \\
2 & $\mathrm{Si}$ & 0.046258 & 0.083086 & 1.914773 \\
3 & $\mathrm{~N}$ & 1.690499 & 0.148976 & 2.534817 \\
4 & $\mathrm{C}$ & 2.659694 & 1.084059 & 1.979120 \\
5 & $\mathrm{H}$ & 3.676952 & 0.670464 & 2.057117 \\
6 & $\mathrm{H}$ & 2.460941 & 1.267829 & 0.918819 \\
7 & $\mathrm{H}$ & 2.661113 & 2.059871 & 2.499994 \\
8 & $\mathrm{C}$ & -1.008362 & 1.576041 & 2.436562 \\
9 & $\mathrm{C}$ & -0.778685 & -1.489945 & 2.566887 \\
10 & $\mathrm{C}$ & 1.986586 & -0.174641 & 3.924014 \\
11 & $\mathrm{H}$ & 3.001249 & -0.593175 & 4.009327 \\
12 & $\mathrm{H}$ & 1.938487 & 0.707172 & 4.590185 \\
13 & $\mathrm{H}$ & 1.289083 & -0.925518 & 4.307043 \\
14 & $\mathrm{H}$ & -0.971763 & -1.450194 & 3.645818 \\
15 & $\mathrm{H}$ & -1.748945 & -1.631316 & 2.072803 \\
16 & $\mathrm{H}$ & -0.168835 & -2.378922 & 2.364624 \\
17 & $\mathrm{H}$ & -1.098611 & 1.641920 & 3.528801 \\
18 & $\mathrm{H}$ & -0.572558 & 2.520502 & 2.085148 \\
19 & $\mathrm{H}$ & -2.025253 & 1.511166 & 2.025960 \\
20 & $\mathrm{H}$ & -0.887317 & -0.141980 & -0.381568 \\
21 & $\mathrm{H}$ & 0.480941 & 0.970302 & -0.412680 \\
22 & $\mathrm{H}$ & 0.766680 & -0.778365 & -0.329594 \\
\hline
\end{tabular}

Total Energy of $\left(\mathrm{Me}_{2} \mathrm{~N}\right) \mathrm{Me}_{2} \mathrm{SiMe}[\mathrm{B} 3 \mathrm{LYP} / 6-31+\mathrm{G}(\mathrm{d})]$ : $\mathrm{E}(\mathrm{RB}+\mathrm{HF}-\mathrm{LYP})=-543.867432669$ Hartree Corrected Zero Point Energy: --543.673640 Hartree 
Table 31. Standard orientation of (MeS)Me ${ }_{2} \mathrm{SiH}[\mathrm{B} 3 \mathrm{LYP} / 6-31+\mathrm{G}(\mathrm{d})]$

\begin{tabular}{ccccc}
$\begin{array}{c}\text { Center } \\
\text { Number }\end{array}$ & $\begin{array}{c}\text { Atom } \\
\text { Type }\end{array}$ & \multicolumn{3}{c}{ Coordinates(Angstroms) } \\
\hline 1 & $\mathrm{Si}$ & -0.717575 & 0.101679 & 0.384120 \\
2 & $\mathrm{C}$ & -2.085405 & -1.133499 & -0.013989 \\
3 & $\mathrm{~S}$ & 1.118628 & -0.771218 & -0.365076 \\
4 & $\mathrm{C}$ & -1.068957 & 1.794198 & -0.378412 \\
5 & $\mathrm{C}$ & 2.378568 & 0.414488 & 0.275828 \\
6 & $\mathrm{H}$ & -0.582190 & 0.270512 & 1.861652 \\
7 & $\mathrm{H}$ & -3.052713 & -0.761887 & 0.347080 \\
8 & $\mathrm{H}$ & -1.897273 & -2.102756 & 0.461226 \\
9 & $\mathrm{H}$ & -2.171304 & -1.296646 & -1.094995 \\
10 & $\mathrm{H}$ & -1.186329 & 1.719544 & -1.466056 \\
11 & $\mathrm{H}$ & -0.262771 & 2.508978 & -0.175036 \\
12 & $\mathrm{H}$ & -1.994470 & 2.215238 & 0.036671 \\
13 & $\mathrm{H}$ & 3.358019 & -0.008893 & 0.040050 \\
14 & $\mathrm{H}$ & 2.297844 & 0.529638 & 1.360555 \\
15 & $\mathrm{H}$ & 2.293955 & 1.391133 & -0.208166 \\
\hline
\end{tabular}

Total Energy of (MeS)Me ${ }_{2} \mathrm{SiH}$ [B3LYP/6-31+G(d)]: E(RB+HF-LYP) $=-808.080125569$ Hartree Corrected Zero Point Energy: -807.957779 Hartree

Table 32. Standard orientation of (MeS) $\mathrm{Me}_{2} \mathrm{SiCl}[\mathrm{B} 3 \mathrm{LYP} / 6-31+\mathrm{G}(\mathrm{d})]$

\begin{tabular}{ccccc}
$\begin{array}{c}\text { Center } \\
\text { Number }\end{array}$ & $\begin{array}{c}\text { Atom } \\
\text { Type }\end{array}$ & \multicolumn{3}{c}{ Coordinates(Angstroms) } \\
\hline 1 & $\mathrm{Si}$ & -0.484416 & -0.342313 & 0.122055 \\
2 & $\mathrm{C}$ & -0.608433 & -0.433859 & 1.995934 \\
3 & $\mathrm{Cl}$ & -1.182934 & 1.575881 & -0.412017 \\
4 & $\mathrm{~S}$ & 1.524223 & -0.620319 & -0.593186 \\
5 & $\mathrm{C}$ & -1.543606 & -1.618211 & -0.755043 \\
6 & $\mathrm{C}$ & 2.446495 & 0.718793 & 0.279564 \\
7 & $\mathrm{H}$ & -1.645772 & -0.279584 & 2.316780 \\
8 & $\mathrm{H}$ & 0.006569 & 0.331212 & 2.482361 \\
9 & $\mathrm{H}$ & -0.277674 & -1.417311 & 2.351201 \\
10 & $\mathrm{H}$ & -1.222636 & -2.634519 & -0.496149 \\
11 & $\mathrm{H}$ & -1.479972 & -1.504052 & -1.842482 \\
12 & $\mathrm{H}$ & -2.594276 & -1.505515 & -0.462506 \\
13 & $\mathrm{H}$ & 3.470803 & 0.683969 & -0.098623 \\
14 & $\mathrm{H}$ & 2.462856 & 0.555017 & 1.359831 \\
15 & $\mathrm{H}$ & 2.017502 & 1.697958 & 0.053338 \\
\hline
\end{tabular}

Total Energy of (MeS)Me ${ }_{2} \mathrm{SiCl}[\mathrm{B} 3 \mathrm{LYP} / 6-31+\mathrm{G}(\mathrm{d})]$ : $\mathrm{E}(\mathrm{RB}+\mathrm{HF}-\mathrm{LYP})=-1267.73629432$ Hartree Corrected Zero Point Energy: -1267.620146 Hartree

Table 33. Standard orientation of (MeS)Me ${ }_{2}$ SiLi [B3LYP/6-31+G(d)]

\begin{tabular}{ccccc}
$\begin{array}{c}\text { Center } \\
\text { Number }\end{array}$ & Atom & \multicolumn{3}{c}{ Coordinates(Angstroms) } \\
\hline 1 & Ty & $\mathrm{X}$ & $\mathrm{Y}$ & $\mathrm{Z}$ \\
\hline 2 & $\mathrm{C}$ & 1.736751 & 0.141603 & 0.400322 \\
3 & $\mathrm{Li}$ & 0.359271 & 1.691141 & -0.675130 \\
4 & $\mathrm{~S}$ & -1.123077 & -0.770466 & 2.856063 \\
5 & $\mathrm{C}$ & 2.019642 & -1.161082 & -0.424643 \\
6 & $\mathrm{C}$ & -2.416616 & 0.416711 & 0.121083 \\
7 & $\mathrm{H}$ & 2.071578 & 2.128463 & -0.392219
\end{tabular}




\begin{tabular}{ccccc}
8 & $\mathrm{H}$ & 0.343888 & 2.472926 & -0.545852 \\
9 & $\mathrm{H}$ & 1.143534 & 1.432287 & -1.742141 \\
10 & $\mathrm{H}$ & 1.985183 & -1.295231 & -1.278929 \\
11 & $\mathrm{H}$ & 1.855696 & -2.140694 & 0.276515 \\
12 & $\mathrm{H}$ & 3.034717 & -0.833506 & 0.074247 \\
13 & $\mathrm{H}$ & -3.389817 & -0.008562 & -0.140360 \\
14 & $\mathrm{H}$ & -2.314611 & 1.384385 & -0.379394 \\
15 & $\mathrm{H}$ & -2.389836 & 0.575700 & 1.207510 \\
\hline
\end{tabular}

Total Energy of (MeS)Me ${ }_{2}$ SiLi [B3LYP/6-31+G(d)]: E(RB+HF-LYP) $=-814.987736155$ Hartree Corrected Zero Point Energy: -814.873624 Hartree

Table 34. Standard orientation of (MeS)Me ${ }_{2} \mathrm{SiMe}[\mathrm{B} 3 \mathrm{LYP} / 6-31+\mathrm{G}(\mathrm{d})]$

\begin{tabular}{ccccc}
$\begin{array}{c}\text { Center } \\
\text { Number }\end{array}$ & $\begin{array}{c}\text { Atom } \\
\text { Type }\end{array}$ & \multicolumn{3}{c}{ Coordinates(Angstroms) } \\
\hline 1 & $\mathrm{Si}$ & -0.663793 & -0.058311 & -0.000033 \\
2 & $\mathrm{C}$ & -0.873680 & -1.131786 & -1.544955 \\
3 & $\mathrm{~S}$ & 1.279109 & 0.920552 & 0.000120 \\
4 & $\mathrm{C}$ & -1.905419 & 1.364175 & -0.001486 \\
5 & $\mathrm{C}$ & 2.448581 & -0.505814 & 0.000097 \\
6 & $\mathrm{C}$ & -0.875105 & -1.129649 & 1.546204 \\
7 & $\mathrm{H}$ & -1.872498 & -1.588454 & -1.563085 \\
8 & $\mathrm{H}$ & -0.141215 & -1.947535 & -1.580221 \\
9 & $\mathrm{H}$ & -0.757067 & -0.536418 & -2.458081 \\
10 & $\mathrm{H}$ & -1.785468 & 1.999363 & -0.887045 \\
11 & $\mathrm{H}$ & -1.789434 & 1.997698 & 0.885793 \\
12 & $\mathrm{H}$ & -2.932378 & 0.976069 & -0.004148 \\
13 & $\mathrm{H}$ & 3.456895 & -0.084372 & -0.000462 \\
14 & $\mathrm{H}$ & 2.328805 & -1.123207 & -0.894301 \\
15 & $\mathrm{H}$ & 2.329525 & -1.122623 & 0.895001 \\
16 & $\mathrm{H}$ & -0.146180 & -1.948608 & 1.580537 \\
17 & $\mathrm{H}$ & -1.875909 & -1.581855 & 1.566783 \\
18 & $\mathrm{H}$ & -0.753978 & -0.534090 & 2.458613 \\
\hline
\end{tabular}

Total Energy of (MeS)Me ${ }_{2}$ SiMe [B3LYP/6-31+G(d)]: E(RB+HF-LYP) $=-847.408992169$ Hartree Corrected Zero Point Energy: -847.257879 Hartree

Table 35. Standard orientation of $(\mathrm{MeO})_{2} \mathrm{MeSiH}[\mathrm{B} 3 \mathrm{LYP} / 6-31+\mathrm{G}(\mathrm{d})]$

\begin{tabular}{ccccc}
$\begin{array}{c}\text { Center } \\
\text { Number }\end{array}$ & $\begin{array}{c}\text { Atom } \\
\text { Type }\end{array}$ & \multicolumn{3}{c}{$\begin{array}{c}\text { Coordinates(Angstroms) } \\
\text { X }\end{array}$} \\
\hline 1 & $\mathrm{H}$ & 0.368744 & 0.795206 & 1.857142 \\
2 & $\mathrm{Si}$ & -0.038649 & 0.499833 & 0.461455 \\
3 & $\mathrm{O}$ & 1.313513 & 0.229235 & -0.474313 \\
4 & $\mathrm{O}$ & -0.976595 & -0.868564 & 0.619446 \\
5 & $\mathrm{C}$ & -0.948717 & 1.906021 & -0.373893 \\
6 & $\mathrm{H}$ & -1.178868 & 1.670152 & -1.419867 \\
7 & $\mathrm{H}$ & -1.889815 & 2.131154 & 0.142188 \\
8 & $\mathrm{H}$ & -0.332687 & 2.813220 & -0.369986 \\
9 & $\mathrm{C}$ & -1.671046 & -1.510545 & -0.443280 \\
10 & $\mathrm{H}$ & -2.175846 & -2.389241 & -0.030597 \\
11 & $\mathrm{H}$ & -2.427633 & -0.847210 & -0.884134 \\
12 & $\mathrm{H}$ & -0.982197 & -1.837371 & -1.233519 \\
13 & $\mathrm{C}$ & 2.368838 & -0.655770 & -0.114940 \\
14 & $\mathrm{H}$ & 3.194709 & -0.494626 & -0.814489 \\
15 & $\mathrm{H}$ & 2.727395 & -0.459231 & 0.905121 \\
16 & $\mathrm{H}$ & 2.047475 & -1.703328 & -0.180613 \\
\hline
\end{tabular}


Total Energy of $(\mathrm{MeO}){ }_{2} \mathrm{MeSiH}[\mathrm{B} 3 \mathrm{LYP} / 6-31+\mathrm{G}(\mathrm{d})]$ : $\mathrm{E}(\mathrm{RB}+\mathrm{HF}-\mathrm{LYP})=-560.368241937$ Hartree Corrected Zero Point Energy: -560.237022 Hartree

Table 36. Standard orientation of $(\mathrm{MeO})_{2} \mathrm{MeSiCl}[\mathrm{B} 3 \mathrm{LYP} / 6-31+\mathrm{G}(\mathrm{d})]$

\begin{tabular}{ccccc}
$\begin{array}{c}\text { Center } \\
\text { Number }\end{array}$ & Atom & \multicolumn{3}{c}{ Coordinates(Angstroms) } \\
\hline 1 & $\mathrm{Cl}$ & -1.772678 & -0.730186 & -0.762009 \\
2 & $\mathrm{Si}$ & -0.012202 & -0.252985 & 0.240746 \\
3 & $\mathrm{O}$ & 1.143958 & -0.362386 & -0.938797 \\
4 & $\mathrm{O}$ & -0.122159 & 1.273168 & 0.858678 \\
5 & $\mathrm{C}$ & 0.261804 & -1.404056 & 1.678306 \\
6 & $\mathrm{H}$ & 1.183391 & -1.145026 & 2.213296 \\
7 & $\mathrm{H}$ & -0.569383 & -1.332728 & 2.387991 \\
8 & $\mathrm{H}$ & 0.337502 & -2.442591 & 1.338125 \\
9 & $\mathrm{C}$ & -0.452672 & 2.458802 & 0.135683 \\
10 & $\mathrm{H}$ & -0.358522 & 3.301161 & 0.826326 \\
11 & $\mathrm{H}$ & 0.226867 & 2.610209 & -0.712015 \\
12 & $\mathrm{H}$ & -1.483348 & 2.409920 & -0.234597 \\
13 & $\mathrm{C}$ & 2.536945 & -0.136368 & -0.732638 \\
14 & $\mathrm{H}$ & 3.027091 & -0.194739 & -1.708324 \\
15 & $\mathrm{H}$ & 2.723308 & 0.854562 & -0.299050 \\
16 & $\mathrm{H}$ & 2.968594 & -0.902341 & -0.075195 \\
\hline
\end{tabular}

Total Energy of $(\mathrm{MeO})_{2} \mathrm{MeSiCl}[\mathrm{B} 3 \mathrm{LYP} / 6-31+\mathrm{G}(\mathrm{d})]$ : $\mathrm{E}(\mathrm{RB}+\mathrm{HF}-\mathrm{LYP})=-1020.02484185$ Hartree Corrected Zero Point Energy: -1019.900135 Hartree

Table 37. Standard orientation of $(\mathrm{MeO})_{2} \mathrm{MeSiLi}[\mathrm{B} 3 \mathrm{LYP} / 6-31+\mathrm{G}(\mathrm{d})]$

\begin{tabular}{ccccc}
$\begin{array}{c}\text { Center } \\
\text { Number }\end{array}$ & $\begin{array}{c}\text { Atom } \\
\text { Type }\end{array}$ & \multicolumn{3}{c}{ Coordinates(Angstroms) } \\
\hline 1 & $\mathrm{Li}$ & -0.733551 & -0.200353 & -2.931475 \\
2 & $\mathrm{Si}$ & -0.278633 & -0.412861 & -0.490493 \\
3 & $\mathrm{O}$ & 1.350532 & -0.471797 & 0.036590 \\
4 & $\mathrm{O}$ & -0.926051 & 1.086410 & 0.037507 \\
5 & $\mathrm{C}$ & -1.004340 & -1.769291 & 0.638176 \\
6 & $\mathrm{H}$ & -0.716598 & -1.623310 & 1.688106 \\
7 & $\mathrm{H}$ & -2.100859 & -1.790695 & 0.579246 \\
8 & $\mathrm{H}$ & -0.632600 & -2.755374 & 0.330664 \\
9 & $\mathrm{C}$ & -0.963551 & 1.499546 & 1.396853 \\
10 & $\mathrm{H}$ & -1.313676 & 2.538103 & 1.431421 \\
11 & $\mathrm{H}$ & -1.656146 & 0.880819 & 1.986094 \\
12 & $\mathrm{H}$ & 0.030045 & 1.449050 & 1.864296 \\
13 & $\mathrm{C}$ & 2.362014 & 0.334680 & -0.523026 \\
14 & $\mathrm{H}$ & 3.336611 & -0.048638 & -0.197373 \\
15 & $\mathrm{H}$ & 2.338291 & 0.317723 & -1.627928 \\
16 & $\mathrm{H}$ & 2.273743 & 1.382698 & -0.199178 \\
\hline
\end{tabular}

Total Energy of (MeO) ${ }_{2}$ MeSiLi [B3LYP/6-31+G(d)]: E(RB+HF-LYP) $=-567.272561807$ Hartree Corrected Zero Point Energy: -567.150003 Hartree

Table 38. Standard orientation of $(\mathrm{MeO})_{2} \mathrm{MeSiMe}[\mathrm{B} 3 \mathrm{LYP} / 6-31+\mathrm{G}(\mathrm{d})]$

\begin{tabular}{ccccc} 
Center & Atom & \multicolumn{3}{c}{ Coordinates(Angstroms) } \\
Number & Type & $\mathrm{X}$ & $\mathrm{Y}$ & $\mathrm{Z}$ \\
\hline 1 & $\mathrm{C}$ & -0.839427 & 1.486342 & -1.329734 \\
2 & $\mathrm{Si}$ & 0.000000 & 0.464955 & 0.000000 \\
3 & $\mathrm{C}$ & 0.839437 & 1.486331 & 1.329737
\end{tabular}




\begin{tabular}{ccccc}
4 & $\mathrm{O}$ & -1.089542 & -0.488492 & 0.836846 \\
5 & $\mathrm{O}$ & 1.089532 & -0.488501 & -0.836847 \\
6 & $\mathrm{C}$ & -1.996043 & -1.402986 & 0.234575 \\
7 & $\mathrm{C}$ & 1.996041 & -1.402987 & -0.234578 \\
8 & $\mathrm{H}$ & -1.291275 & 0.847410 & -2.097957 \\
9 & $\mathrm{H}$ & -1.627698 & 2.120974 & -0.906570 \\
10 & $\mathrm{H}$ & -0.113567 & 2.137083 & -1.832293 \\
11 & $\mathrm{H}$ & 0.113587 & 2.137098 & 1.832279 \\
12 & $\mathrm{H}$ & 1.627732 & 2.120936 & 0.906580 \\
13 & $\mathrm{H}$ & 1.291253 & 0.847393 & 2.097974 \\
14 & $\mathrm{H}$ & -1.473564 & -2.133876 & -0.397475 \\
15 & $\mathrm{H}$ & -2.514999 & -1.938859 & 1.035650 \\
16 & $\mathrm{H}$ & -2.744534 & -0.879529 & -0.376166 \\
17 & $\mathrm{H}$ & 1.473568 & -2.133883 & 0.397471 \\
18 & $\mathrm{H}$ & 2.515001 & -1.938856 & -1.035653 \\
19 & $\mathrm{H}$ & 2.744528 & -0.879525 & 0.376165 \\
\hline
\end{tabular}

Total Energy of $(\mathrm{MeO})_{2} \mathrm{MeSiMe}[\mathrm{B} 3 \mathrm{LYP} / 6-31+\mathrm{G}(\mathrm{d})]$ : $E(\mathrm{RB}+\mathrm{HF}-\mathrm{LYP})=-599.699256846$ Hartree Corrected Zero Point Energy: -599.539601 Hartree

Table 39. Standard orientation of $\left(\mathrm{Me}_{2} \mathrm{~N}\right)_{2} \mathrm{MeSiH}[\mathrm{B} 3 \mathrm{LYP} / 6-31+\mathrm{G}(\mathrm{d})]$

\begin{tabular}{ccccc}
$\begin{array}{c}\text { Center } \\
\text { Number }\end{array}$ & $\begin{array}{c}\text { Atom } \\
\text { Type }\end{array}$ & \multicolumn{3}{c}{$\begin{array}{c}\text { Coordinates(Angstroms) } \\
\text { X }\end{array}$} \\
\hline 1 & $\mathrm{H}$ & 0.241456 & 0.414451 & -0.263038 \\
2 & $\mathrm{Si}$ & 0.151170 & 0.264934 & 1.212570 \\
3 & $\mathrm{~N}$ & 1.827094 & 0.142019 & 1.708170 \\
4 & $\mathrm{C}$ & 2.877119 & 0.872497 & 1.011505 \\
5 & $\mathrm{H}$ & 3.793452 & 0.264512 & 0.950351 \\
6 & $\mathrm{H}$ & 2.565725 & 1.108694 & -0.011247 \\
7 & $\mathrm{H}$ & 3.142203 & 1.818987 & 1.516217 \\
8 & $\mathrm{~N}$ & -0.804726 & 1.638362 & 1.729304 \\
9 & $\mathrm{C}$ & -1.480701 & 1.706656 & 3.016571 \\
10 & $\mathrm{H}$ & -2.443754 & 2.231708 & 2.917866 \\
11 & $\mathrm{H}$ & -1.691164 & 0.703572 & 3.400063 \\
12 & $\mathrm{H}$ & -0.889169 & 2.245811 & 3.778526 \\
13 & $\mathrm{C}$ & -0.749344 & -1.304747 & 1.740169 \\
14 & $\mathrm{C}$ & 2.223772 & -0.245424 & 3.054462 \\
15 & $\mathrm{H}$ & 3.134845 & -0.863412 & 3.025522 \\
16 & $\mathrm{H}$ & 2.436897 & 0.626173 & 3.699093 \\
17 & $\mathrm{H}$ & 1.440713 & -0.837887 & 3.538389 \\
18 & $\mathrm{C}$ & -0.572742 & 2.955980 & 1.153225 \\
19 & $\mathrm{H}$ & -1.523570 & 3.500137 & 1.041070 \\
20 & $\mathrm{H}$ & 0.094578 & 3.580212 & 1.774260 \\
21 & $\mathrm{H}$ & -0.123115 & 2.865951 & 0.158324 \\
22 & $\mathrm{H}$ & -0.758817 & -1.464651 & 2.824684 \\
23 & $\mathrm{H}$ & -1.791607 & -1.278352 & 1.401232 \\
24 & $\mathrm{H}$ & -0.263793 & -2.176777 & 1.286640 \\
\hline
\end{tabular}

Total Energy of $\left(\mathrm{Me}_{2} \mathrm{~N}\right)_{2} \mathrm{MeSiH}[\mathrm{B} 3 \mathrm{LYP} / 6-31+\mathrm{G}(\mathrm{d})]$ : $\mathrm{E}(\mathrm{RB}+\mathrm{HF}-\mathrm{LYP})=-599.211549221$ Hartree Corrected Zero Point Energy: -598.999625 Hartree

Table 40. Standard orientation of $\left(\mathrm{Me}_{2} \mathrm{~N}\right)_{2} \mathrm{MeSiCl}[\mathrm{B} 3 \mathrm{LYP} / 6-31+\mathrm{G}(\mathrm{d})]$

\begin{tabular}{ccccc}
$\begin{array}{c}\text { Center } \\
\text { Number }\end{array}$ & Atom & \multicolumn{3}{c}{ Coordinates(Angstroms) } \\
\hline 1 & $\mathrm{Cl}$ & -0.267677 & 2.032671 & -0.809789 \\
2 & $\mathrm{Si}$ & 0.017023 & 0.260864 & 0.325151 \\
3 & $\mathrm{C}$ & 0.189152 & 0.850570 & 2.101497
\end{tabular}




\begin{tabular}{ccccc}
4 & $\mathrm{H}$ & 0.392780 & 0.015114 & 2.783017 \\
5 & $\mathrm{H}$ & 0.979104 & 1.600085 & 2.222818 \\
6 & $\mathrm{H}$ & -0.753766 & 1.315103 & 2.413685 \\
7 & $\mathrm{~N}$ & 1.431828 & -0.596996 & -0.163240 \\
8 & $\mathrm{C}$ & 2.741620 & -0.388725 & 0.444426 \\
9 & $\mathrm{C}$ & 1.523653 & -1.186546 & -1.497020 \\
10 & $\mathrm{~N}$ & -1.354632 & -0.738436 & -0.018557 \\
11 & $\mathrm{C}$ & -1.350670 & -2.145635 & 0.375382 \\
12 & $\mathrm{C}$ & -2.695944 & -0.254069 & -0.323582 \\
13 & $\mathrm{H}$ & 2.092190 & -0.543162 & -2.188092 \\
14 & $\mathrm{H}$ & 2.030530 & -2.161855 & -1.448351 \\
15 & $\mathrm{H}$ & 0.525606 & -1.339999 & -1.915935 \\
16 & $\mathrm{H}$ & 3.363137 & 0.309696 & -0.140521 \\
17 & $\mathrm{H}$ & 2.645816 & 0.007813 & 1.458248 \\
18 & $\mathrm{H}$ & 3.283811 & -1.343715 & 0.509656 \\
19 & $\mathrm{H}$ & -3.142896 & -0.858985 & -1.127040 \\
20 & $\mathrm{H}$ & -3.363437 & -0.318834 & 0.552547 \\
21 & $\mathrm{H}$ & -2.669045 & 0.783690 & -0.661085 \\
22 & $\mathrm{H}$ & -1.769886 & -2.769044 & -0.429351 \\
23 & $\mathrm{H}$ & -0.331732 & -2.489636 & 0.572685 \\
24 & $\mathrm{H}$ & -1.957275 & -2.319327 & 1.280383 \\
\hline
\end{tabular}

Total Energy of $\left(\mathrm{Me}_{2} \mathrm{~N}\right)_{2} \mathrm{MeSiCl}[\mathrm{B} 3 \mathrm{LYP} / 6-31+\mathrm{G}(\mathrm{d})]$ : $\mathrm{E}(\mathrm{RB}+\mathrm{HF}-\mathrm{LYP})=-1058.87203060$ Hartree Corrected Zero Point Energy: -1058.666209 Hartree

Table 41. Standard orientation of $\left(\mathrm{Me}_{2} \mathrm{~N}\right)_{2} \mathrm{MeSiLi}[\mathrm{B} 3 \mathrm{LYP} / 6-31+\mathrm{G}(\mathrm{d})]$

\begin{tabular}{|c|c|c|c|c|}
\hline \multirow{2}{*}{$\begin{array}{l}\text { Center } \\
\text { Number }\end{array}$} & \multirow{2}{*}{$\begin{array}{l}\text { Atom } \\
\text { Type }\end{array}$} & \multicolumn{3}{|c|}{ Coordinates (Angstroms) } \\
\hline & & $\mathrm{X}$ & $\mathrm{Y}$ & Z \\
\hline 1 & $\mathrm{Li}$ & 0.345080 & -0.396793 & 0.286899 \\
\hline 2 & $\mathrm{Si}$ & -0.035910 & -0.024830 & 2.754407 \\
\hline 3 & $\mathrm{~N}$ & 1.477203 & 0.390285 & 3.640833 \\
\hline 4 & C & 2.180625 & 1.587421 & 3.211228 \\
\hline 5 & $\mathrm{H}$ & 2.798616 & 1.423755 & 2.300569 \\
\hline 6 & $\mathrm{H}$ & 1.465607 & 2.387233 & 2.994907 \\
\hline 7 & $\mathrm{H}$ & 2.860553 & 1.940822 & 4.001829 \\
\hline 8 & $\mathrm{~N}$ & -1.062385 & 1.390958 & 3.069239 \\
\hline 9 & C & -2.007709 & 1.925999 & 2.113872 \\
\hline 10 & $\mathrm{H}$ & -2.006552 & 3.030242 & 2.126281 \\
\hline 11 & $\mathrm{H}$ & -1.746858 & 1.603272 & 1.097448 \\
\hline 12 & $\mathrm{H}$ & -3.048883 & 1.602297 & 2.307816 \\
\hline 13 & C & -0.727959 & -1.513173 & 3.757510 \\
\hline 14 & C & 2.406345 & -0.671880 & 3.986046 \\
\hline 15 & $\mathrm{H}$ & 3.016444 & -1.017583 & 3.122800 \\
\hline 16 & $\mathrm{H}$ & 3.113561 & -0.327608 & 4.756405 \\
\hline 17 & $\mathrm{H}$ & 1.874532 & -1.538404 & 4.389397 \\
\hline 18 & C & -1.335439 & 1.835792 & 4.428077 \\
\hline 19 & $\mathrm{H}$ & -1.328330 & 2.937530 & 4.489552 \\
\hline 20 & $\mathrm{H}$ & -2.322613 & 1.495486 & 4.793981 \\
\hline 21 & $\mathrm{H}$ & -0.565985 & 1.459010 & 5.108627 \\
\hline 22 & $\mathrm{H}$ & -0.712687 & -1.330274 & 4.841877 \\
\hline 23 & $\mathrm{H}$ & -1.772523 & -1.681530 & 3.461508 \\
\hline 24 & $\mathrm{H}$ & -0.185191 & -2.448054 & 3.564027 \\
\hline
\end{tabular}

Total Energy of $\left(\mathrm{Me}_{2} \mathrm{~N}\right)_{2}$ MeSiLi [B3LYP/6-31+G(d)]: E(RB+HF-LYP) $=-606.110723692$ Hartree Corrected Zero Point Energy: -605.907958 Hartree

Table 42. Standard orientation of $\left(\mathrm{Me}_{2} \mathrm{~N}\right)_{2} \mathrm{MeSiMe}[\mathrm{B} 3 \mathrm{LYP} / 6-31+\mathrm{G}(\mathrm{d})]$ 


\begin{tabular}{ccccc}
$\begin{array}{c}\text { Center } \\
\text { Number }\end{array}$ & Atom & \multicolumn{3}{c}{ Coordinates (Angstroms) } \\
\hline 1 & $\mathrm{C}$ & 0.012465 & -0.182630 & 0.251627 \\
2 & $\mathrm{Si}$ & -0.057691 & 0.048994 & 2.130344 \\
3 & $\mathrm{~N}$ & 1.504226 & 0.369505 & 2.848510 \\
4 & $\mathrm{C}$ & 2.236259 & 1.581858 & 2.506369 \\
5 & $\mathrm{H}$ & 3.022905 & 1.397388 & 1.752707 \\
6 & $\mathrm{H}$ & 1.552825 & 2.340019 & 2.112046 \\
7 & $\mathrm{H}$ & 2.727739 & 2.003028 & 3.397933 \\
8 & $\mathrm{~N}$ & -1.044643 & 1.455325 & 2.458210 \\
9 & $\mathrm{C}$ & -2.090090 & 1.942105 & 1.571905 \\
10 & $\mathrm{H}$ & -2.110009 & 3.043682 & 1.567845 \\
11 & $\mathrm{H}$ & -1.918868 & 1.611225 & 0.544239 \\
12 & $\mathrm{H}$ & -3.095672 & 1.598686 & 1.876647 \\
13 & $\mathrm{C}$ & -0.734147 & -1.543659 & 2.901156 \\
14 & $\mathrm{C}$ & 2.361702 & -0.650805 & 3.430495 \\
15 & $\mathrm{H}$ & 3.148815 & -0.989357 & 2.732066 \\
16 & $\mathrm{H}$ & 2.868742 & -0.262204 & 4.328229 \\
17 & $\mathrm{H}$ & 1.780827 & -1.526156 & 3.732581 \\
18 & $\mathrm{C}$ & -1.202454 & 1.942745 & 3.822332 \\
19 & $\mathrm{H}$ & -1.195865 & 3.044220 & 3.843527 \\
20 & $\mathrm{H}$ & -2.152069 & 1.610181 & 4.278934 \\
21 & $\mathrm{H}$ & -0.377565 & 1.591476 & 4.449769 \\
22 & $\mathrm{H}$ & -0.753365 & -1.500703 & 3.997335 \\
23 & $\mathrm{H}$ & -1.767610 & -1.692475 & 2.561369 \\
24 & $\mathrm{H}$ & -0.166477 & -2.436330 & 2.608657 \\
25 & $\mathrm{H}$ & -0.948232 & -0.492486 & -0.179269 \\
26 & $\mathrm{H}$ & 0.337541 & 0.727177 & -0.268295 \\
27 & $\mathrm{H}$ & 0.740253 & -0.970834 & 0.017672 \\
\hline & & & &
\end{tabular}

Total Energy of $\left(\mathrm{Me}_{2} \mathrm{~N}\right)_{2} \mathrm{MeSiMe}[\mathrm{B} 3 \mathrm{LYP} / 6-31+\mathrm{G}(\mathrm{d})]$ : $\mathrm{E}(\mathrm{RB}+\mathrm{HF}-\mathrm{LYP})=-638.539853012$ Hartree Corrected Zero Point Energy: -638.299581 Hartree

Table 43. Standard orientation of (MeS) ${ }_{2} \mathrm{MeSiH}[\mathrm{B} 3 \mathrm{LYP} / 6-31+\mathrm{G}(\mathrm{d})]$

\begin{tabular}{ccccc}
$\begin{array}{c}\text { Center } \\
\text { Number }\end{array}$ & Atom & \multicolumn{3}{c}{ Coordinates(Angstroms) } \\
\hline 1 & $\mathrm{H}$ & -0.335733 & 0.858699 & -1.992011 \\
2 & $\mathrm{Si}$ & 0.026901 & 0.709011 & -0.557513 \\
3 & $\mathrm{~S}$ & -1.716400 & 0.185200 & 0.604050 \\
4 & $\mathrm{~S}$ & 1.516524 & -0.853403 & -0.628793 \\
5 & $\mathrm{C}$ & 0.668848 & 2.319786 & 0.178379 \\
6 & $\mathrm{H}$ & 0.946137 & 2.203833 & 1.232268 \\
7 & $\mathrm{H}$ & 1.553961 & 2.657913 & -0.372600 \\
8 & $\mathrm{H}$ & -0.096754 & 3.102222 & 0.115867 \\
9 & $\mathrm{C}$ & 1.979576 & -1.027130 & 1.149556 \\
10 & $\mathrm{H}$ & 2.616879 & -1.911635 & 1.221756 \\
11 & $\mathrm{H}$ & 2.541389 & -0.157402 & 1.499444 \\
12 & $\mathrm{H}$ & 1.093752 & -1.174118 & 1.773001 \\
13 & $\mathrm{C}$ & -2.330743 & -1.298162 & -0.305094 \\
14 & $\mathrm{H}$ & -3.194217 & -1.671492 & 0.250648 \\
15 & $\mathrm{H}$ & -2.645491 & -1.033700 & -1.318067 \\
16 & $\mathrm{H}$ & -1.564605 & -2.076184 & -0.346295 \\
\hline
\end{tabular}

Total Energy of (MeS) $)_{2} \mathrm{MeSiH}$ [B3LYP/6-31+G(d)]: E(RB+HF-LYP) $=-1206.29000702$ Hartree Corrected Zero Point Energy: -1206.164488 Hartree

Table 44. Standard orientation of (MeS) ${ }_{2} \mathrm{MeSiCl}[\mathrm{B} 3 \mathrm{LYP} / 6-31+\mathrm{G}(\mathrm{d})]$ 


\begin{tabular}{ccccc}
$\begin{array}{c}\text { Center } \\
\text { Number }\end{array}$ & Atom & \multicolumn{3}{c}{ Coordinates(Angstroms) } \\
\hline 1 & $\mathrm{Cl}$ & -1.363093 & -1.672940 & -0.769199 \\
2 & $\mathrm{Si}$ & -0.079600 & -0.440497 & 0.333823 \\
3 & $\mathrm{~S}$ & 1.617934 & -0.229593 & -0.975707 \\
4 & $\mathrm{~S}$ & -1.025971 & 1.426249 & 0.810611 \\
5 & $\mathrm{C}$ & 0.312132 & -1.271410 & 1.968443 \\
6 & $\mathrm{H}$ & 1.010905 & -0.670928 & 2.562574 \\
7 & $\mathrm{H}$ & -0.602565 & -1.412728 & 2.555223 \\
8 & $\mathrm{H}$ & 0.764837 & -2.252671 & 1.788798 \\
9 & $\mathrm{C}$ & -1.458704 & 2.085141 & -0.857887 \\
10 & $\mathrm{H}$ & -1.857419 & 3.089660 & -0.698864 \\
11 & $\mathrm{H}$ & -0.575096 & 2.141264 & -1.497825 \\
12 & $\mathrm{H}$ & -2.222400 & 1.462935 & -1.329495 \\
13 & $\mathrm{C}$ & 2.718980 & 0.841825 & 0.050266 \\
14 & $\mathrm{H}$ & 3.555967 & 1.122854 & -0.593504 \\
15 & $\mathrm{H}$ & 2.199848 & 1.746640 & 0.376180 \\
16 & $\mathrm{H}$ & 3.107042 & 0.300075 & 0.916390 \\
\hline
\end{tabular}

Total Energy of (MeS) ${ }_{2} \mathrm{MeSiCl}$ [B3LYP/6-31+G(d)]: E(RB+HF-LYP) $=-1665.94225475$ Hartree Corrected Zero Point Energy: -1665.823279 Hartree

Table 45. Standard orientation of (MeS) ${ }_{2}$ MeSiLi [B3LYP/6-31+G(d)]

\begin{tabular}{ccccc}
$\begin{array}{c}\text { Center } \\
\text { Number }\end{array}$ & Atomic & \multicolumn{3}{c}{ Coordinates (Angstroms) } \\
\hline 1 & Type & X & Y & Z \\
2 & Si & 0.529438 & 1.160848 & 2.882086 \\
3 & $\mathrm{~S}$ & 0.026937 & 0.806507 & 0.480266 \\
4 & $\mathrm{~S}$ & -1.541503 & -0.001378 & -0.799337 \\
5 & $\mathrm{C}$ & -0.658254 & -0.735222 & 0.698823 \\
6 & $\mathrm{H}$ & -0.917131 & 1.749354 & -0.699824 \\
7 & $\mathrm{H}$ & -1.561027 & 2.601584 & -1.687601 \\
8 & $\mathrm{H}$ & 0.082796 & 2.946945 & -0.846917 \\
9 & $\mathrm{C}$ & -2.095048 & -1.181040 & -1.005978 \\
10 & $\mathrm{H}$ & -2.707528 & -2.083806 & -0.929406 \\
11 & $\mathrm{H}$ & -2.694799 & -0.379289 & -1.445008 \\
12 & $\mathrm{H}$ & -1.230025 & -1.386158 & -1.642976 \\
13 & $\mathrm{C}$ & 2.389750 & -1.274043 & 0.296241 \\
14 & $\mathrm{H}$ & 3.178037 & -1.776283 & -0.271577 \\
15 & $\mathrm{H}$ & 2.839011 & -0.820496 & 1.187938 \\
16 & $\mathrm{H}$ & 1.644932 & -2.015136 & 0.600502 \\
\hline
\end{tabular}

Total Energy of $(\mathrm{MeS})_{2} \mathrm{MeSiLi}[\mathrm{B} 3 \mathrm{LYP} / 6-31+\mathrm{G}(\mathrm{d})]$ : $\mathrm{E}(\mathrm{RB}+\mathrm{HF}-\mathrm{LYP})=-1213.20763646$ Hartree Corrected Zero Point Energy: -1213.090307 Hartree

Table 46. Standard orientation of (MeS) $)_{2}$ MeSiMe $[B 3 L Y P / 6-31+G(d)]$

\begin{tabular}{ccccc}
$\begin{array}{c}\text { Center } \\
\text { Number }\end{array}$ & Atom & \multicolumn{3}{c}{ Coordinates (Angstroms) } \\
\hline 1 & C & -0.626889 & 1.777074 & -1.430292 \\
2 & Si & 0.000081 & 0.719780 & -0.000009 \\
3 & S & -1.570568 & -0.495214 & 0.871967 \\
4 & S & 1.570608 & -0.495271 & -0.872044 \\
5 & C & 0.627039 & 1.776851 & 1.430446 \\
6 & H & 0.994609 & 1.167540 & 2.263511 \\
7 & H & 1.448306 & 2.418868 & 1.089974 \\
8 & H & -0.174660 & 2.419618 & 1.814440 \\
9 & C & 2.168443 & -1.463204 & 0.579555
\end{tabular}




\begin{tabular}{ccccc}
10 & $\mathrm{H}$ & 2.855074 & -2.219680 & 0.191886 \\
11 & $\mathrm{H}$ & 2.706715 & -0.825253 & 1.285205 \\
12 & $\mathrm{H}$ & 1.336656 & -1.959546 & 1.086100 \\
13 & $\mathrm{C}$ & -2.168734 & -1.463002 & -0.579586 \\
14 & $\mathrm{H}$ & -2.856531 & -2.218430 & -0.191932 \\
15 & $\mathrm{H}$ & -2.705792 & -0.824724 & -1.285847 \\
16 & $\mathrm{H}$ & -1.337277 & -1.960655 & -1.085388 \\
17 & $\mathrm{H}$ & 0.175168 & 2.418616 & -1.815560 \\
18 & $\mathrm{H}$ & -0.996272 & 1.167828 & -2.262600 \\
19 & $\mathrm{H}$ & -1.446917 & 2.420338 & -1.089162 \\
\hline
\end{tabular}

Total Energy of $(\mathrm{MeS})_{2} \mathrm{MeSiMe}[\mathrm{B} 3 \mathrm{LYP} / 6-31+\mathrm{G}(\mathrm{d})]$ : $E(\mathrm{RB}+\mathrm{HF}-\mathrm{LYP})=-1245.62014819$ Hartree Corrected Zero Point Energy: -1245.465911 Hartree

Table 47. Standard orientation of $(\mathrm{MeO})_{3} \mathrm{SiCl}[\mathrm{B} 3 \mathrm{LYP} / 6-31+\mathrm{G}(\mathrm{d})]$

\begin{tabular}{ccccc}
$\begin{array}{c}\text { Center } \\
\text { Number }\end{array}$ & $\begin{array}{c}\text { Atom } \\
\text { Type }\end{array}$ & \multicolumn{3}{c}{ Coordinates(Angstroms) } \\
\hline 1 & $\mathrm{Cl}$ & -0.452443 & 0.784133 & 0.061739 \\
2 & $\mathrm{Si}$ & -0.414427 & 0.502416 & 2.113645 \\
3 & $\mathrm{O}$ & 1.050177 & -0.113973 & 2.533906 \\
4 & $\mathrm{C}$ & 2.330677 & 0.415689 & 2.190955 \\
5 & $\mathrm{H}$ & 3.084547 & -0.240475 & 2.633944 \\
6 & $\mathrm{H}$ & 2.464447 & 0.436395 & 1.103129 \\
7 & $\mathrm{H}$ & 2.455993 & 1.429294 & 2.590300 \\
8 & $\mathrm{O}$ & -0.741435 & 1.968114 & 2.763587 \\
9 & $\mathrm{C}$ & -1.090861 & 2.193581 & 4.132672 \\
10 & $\mathrm{H}$ & -1.266933 & 3.266083 & 4.248541 \\
11 & $\mathrm{H}$ & -2.001686 & 1.644663 & 4.394821 \\
12 & $\mathrm{H}$ & -0.276983 & 1.888889 & 4.801953 \\
13 & $\mathrm{O}$ & -1.552234 & -0.563334 & 2.606263 \\
14 & $\mathrm{C}$ & -1.556037 & -1.970967 & 2.363152 \\
15 & $\mathrm{H}$ & -0.615845 & -2.426808 & 2.692593 \\
16 & $\mathrm{H}$ & -2.386369 & -2.397007 & 2.932329 \\
17 & $\mathrm{H}$ & -1.709581 & -2.176545 & 1.297104 \\
\hline
\end{tabular}

Total Energy of $(\mathrm{MeO})_{3} \mathrm{SiCl}[\mathrm{B} 3 \mathrm{LYP} / 6-31+\mathrm{G}(\mathrm{d})]$ : $\mathrm{E}(\mathrm{RB}+\mathrm{HF}-\mathrm{LYP})=-1095.27384104$ Hartree Corrected Zero Point Energy: -1095.142793 Hartree

Table 48. Standard orientation of $(\mathrm{MeO})_{3} \mathrm{SiLi}[\mathrm{B} 3 \mathrm{LYP} / 6-31+\mathrm{G}(\mathrm{d})]$

\begin{tabular}{ccccc} 
Center & Atomic & \multicolumn{3}{c}{ Coordinates(Angstroms) } \\
Number & Number & $\mathrm{X}$ & $\mathrm{Y}$ & $\mathrm{Z}$ \\
\hline 1 & $\mathrm{Li}$ & -0.136050 & -0.691519 & -1.060039 \\
2 & $\mathrm{Si}$ & 0.111429 & -0.043959 & 1.320069 \\
3 & $\mathrm{O}$ & 1.699402 & -0.506844 & 1.676241 \\
4 & $\mathrm{C}$ & 2.371431 & -0.176750 & 2.889720 \\
5 & $\mathrm{H}$ & 3.344121 & -0.681852 & 2.885448 \\
6 & $\mathrm{H}$ & 2.528787 & 0.905986 & 2.967785 \\
7 & $\mathrm{H}$ & 1.801432 & -0.512490 & 3.766543 \\
8 & $\mathrm{O}$ & 0.097486 & 1.596331 & 1.746582 \\
9 & $\mathrm{C}$ & -1.085578 & 2.343734 & 1.949883 \\
10 & $\mathrm{H}$ & -0.801678 & 3.375548 & 2.187799 \\
11 & $\mathrm{H}$ & -1.717656 & 2.362478 & 1.045696 \\
12 & $\mathrm{H}$ & -1.680026 & 1.936347 & 2.777972 \\
13 & $\mathrm{O}$ & -0.914861 & -0.677955 & 2.527758 \\
14 & $\mathrm{C}$ & -1.314907 & -2.029242 & 2.577823 \\
15 & $\mathrm{H}$ & -0.517740 & -2.672334 & 2.979361 \\
16 & $\mathrm{H}$ & -1.595387 & -2.416392 & 1.582160
\end{tabular}


Total Energy of $(\mathrm{MeO})_{3} \mathrm{SiLi}[\mathrm{B} 3 \mathrm{LYP} / 6-31+\mathrm{G}(\mathrm{d})]$ : $\mathrm{E}(\mathrm{RB}+\mathrm{HF}-\mathrm{LYP})=-642.527137091$ Hartree Corrected Zero Point Energy: -642.398638 Hartree

Table 49. Standard orientation of $\left(\mathrm{Me}_{2} \mathrm{~N}\right)_{3} \mathrm{SiCl}[\mathrm{B} 3 \mathrm{LYP} / 6-31+\mathrm{G}(\mathrm{d})]$

\begin{tabular}{|c|c|c|c|c|}
\hline \multirow{2}{*}{$\begin{array}{l}\text { Center } \\
\text { Number }\end{array}$} & \multirow{2}{*}{$\begin{array}{l}\text { Atomic } \\
\text { Number }\end{array}$} & \multicolumn{3}{|c|}{ Coordinates(Angstroms) } \\
\hline & & $\mathrm{X}$ & Y & Z \\
\hline 1 & $\mathrm{Cl}$ & -0.026437 & 1.079083 & 0.585545 \\
\hline 2 & $\mathrm{Si}$ & -0.089679 & 0.398685 & 2.604362 \\
\hline 3 & $\mathrm{~N}$ & 1.534755 & 0.244810 & 3.147174 \\
\hline 4 & C & 2.561915 & -0.412269 & 2.347099 \\
\hline 5 & $\mathrm{H}$ & 2.988225 & -1.275075 & 2.883810 \\
\hline 6 & $\mathrm{H}$ & 2.149395 & -0.770811 & 1.400390 \\
\hline 7 & $\mathrm{H}$ & 3.385596 & 0.280668 & 2.114158 \\
\hline 8 & $\mathrm{~N}$ & -0.910920 & 1.589173 & 3.542546 \\
\hline 9 & C & -2.226921 & 1.384773 & 4.133483 \\
\hline 10 & $\mathrm{H}$ & -3.019258 & 1.890812 & 3.556369 \\
\hline 11 & $\mathrm{H}$ & -2.463941 & 0.319021 & 4.181648 \\
\hline 12 & $\mathrm{H}$ & -2.253565 & 1.786353 & 5.158127 \\
\hline 13 & $\mathrm{~N}$ & -0.909403 & -1.115222 & 2.569569 \\
\hline 14 & C & -0.505199 & -2.257278 & 3.379659 \\
\hline 15 & $\mathrm{H}$ & -0.332233 & -3.142868 & 2.747629 \\
\hline 16 & $\mathrm{H}$ & 0.419504 & -2.037338 & 3.919211 \\
\hline 17 & $\mathrm{H}$ & -1.280472 & -2.521399 & 4.117938 \\
\hline 18 & C & 2.035987 & 0.755653 & 4.415772 \\
\hline 19 & $\mathrm{H}$ & 2.456802 & -0.057840 & 5.029274 \\
\hline 20 & $\mathrm{H}$ & 2.834761 & 1.498436 & 4.257735 \\
\hline 21 & $\mathrm{H}$ & 1.230908 & 1.228809 & 4.982368 \\
\hline 22 & C & -2.105705 & -1.372454 & 1.776876 \\
\hline 23 & $\mathrm{H}$ & -1.950120 & -2.227679 & 1.100903 \\
\hline 24 & $\mathrm{H}$ & -2.969628 & -1.606035 & 2.420566 \\
\hline 25 & $\mathrm{H}$ & -2.361749 & -0.505619 & 1.162576 \\
\hline 26 & C & -0.531686 & 2.996732 & 3.467663 \\
\hline 27 & $\mathrm{H}$ & -1.235709 & 3.574785 & 2.847475 \\
\hline 28 & $\mathrm{H}$ & -0.515822 & 3.445910 & 4.472454 \\
\hline 29 & $\mathrm{H}$ & 0.465269 & 3.107442 & 3.031264 \\
\hline
\end{tabular}

Total Energy of $\left(\mathrm{Me}_{2} \mathrm{~N}\right)_{3} \mathrm{SiCl}[\mathrm{B} 3 \mathrm{LYP} / 6-31+\mathrm{G}(\mathrm{d})]$ : $\mathrm{E}(\mathrm{RB}+\mathrm{HF}-\mathrm{LYP})=-1153.54425081$ Hartree Corrected Zero Point Energy: -1153.292632 Hartree

Table 50. Standard orientation of $\left(\mathrm{Me}_{2} \mathrm{~N}\right)_{3} \mathrm{SiLi}[\mathrm{B} 3 \mathrm{LYP} / 6-31+\mathrm{G}(\mathrm{d})]$

\begin{tabular}{ccccc}
$\begin{array}{c}\text { Center } \\
\text { Number }\end{array}$ & $\begin{array}{c}\text { Atom } \\
\text { Type }\end{array}$ & \multicolumn{3}{c}{ Coordinates(Angstroms) } \\
\hline 1 & $\mathrm{Xi}$ & -0.503827 & -0.567864 & 0.363971 \\
2 & $\mathrm{Si}$ & -0.209990 & -0.033343 & 2.793223 \\
3 & $\mathrm{~N}$ & 1.494733 & 0.263258 & 3.228321 \\
4 & $\mathrm{C}$ & 2.422422 & 0.875448 & 2.299368 \\
5 & $\mathrm{H}$ & 3.416324 & 0.397332 & 2.352592 \\
6 & $\mathrm{H}$ & 2.056935 & 0.771258 & 1.269126 \\
7 & $\mathrm{H}$ & 2.572130 & 1.955689 & 2.490179 \\
8 & $\mathrm{~N}$ & -1.330593 & 1.229629 & 3.366625 \\
9 & $\mathrm{C}$ & -1.849988 & 2.258080 & 2.489496 \\
10 & $\mathrm{H}$ & -1.316330 & 3.222626 & 2.593347 \\
11 & $\mathrm{H}$ & -1.763661 & 1.944379 & 1.440841 \\
12 & $\mathrm{H}$ & -2.917183 & 2.455966 & 2.692522 \\
13 & $\mathrm{~N}$ & -0.607911 & -1.381443 & 3.914372
\end{tabular}




\begin{tabular}{ccccc}
14 & $\mathrm{C}$ & 0.251928 & -2.554520 & 3.912336 \\
15 & $\mathrm{H}$ & 0.000595 & -3.266853 & 3.097135 \\
16 & $\mathrm{H}$ & 1.298179 & -2.262192 & 3.790775 \\
17 & $\mathrm{H}$ & 0.153940 & -3.107889 & 4.859721 \\
18 & $\mathrm{C}$ & 1.957821 & 0.374602 & 4.603788 \\
19 & $\mathrm{H}$ & 2.944299 & -0.105313 & 4.727315 \\
20 & $\mathrm{H}$ & 2.066998 & 1.427105 & 4.926269 \\
21 & $\mathrm{H}$ & 1.255358 & -0.119539 & 5.280647 \\
22 & $\mathrm{C}$ & -2.002063 & -1.761091 & 4.078869 \\
23 & $\mathrm{H}$ & -2.363499 & -2.425461 & 3.264615 \\
24 & $\mathrm{H}$ & -2.143409 & -2.309308 & 5.023734 \\
25 & $\mathrm{H}$ & -2.641247 & -0.874807 & 4.095607 \\
26 & $\mathrm{C}$ & -1.439689 & 1.606872 & 4.768376 \\
27 & $\mathrm{H}$ & -0.857595 & 2.517191 & 5.004883 \\
28 & $\mathrm{H}$ & -2.489321 & 1.813819 & 5.040547 \\
29 & $\mathrm{H}$ & -1.080687 & 0.795985 & 5.408463 \\
\hline
\end{tabular}

Total Energy of $\left(\mathrm{Me}_{2} \mathrm{~N}\right)_{3} \mathrm{SiLi}[\mathrm{B} 3 \mathrm{LYP} / 6-31+\mathrm{G}(\mathrm{d})]$ : $\mathrm{E}(\mathrm{RB}+\mathrm{HF}-\mathrm{LYP})=-700.784116348$ Hartree Corrected Zero Point Energy: -700.535238 Hartree

Table 51. Standard orientation of $(\mathrm{MeS})_{3} \mathrm{SiCl}[\mathrm{B} 3 \mathrm{LYP} / 6-31+\mathrm{G}(\mathrm{d})]$

\begin{tabular}{ccccc}
$\begin{array}{c}\text { Center } \\
\text { Number }\end{array}$ & Atom & \multicolumn{3}{c}{ Coordinates (Angstroms) } \\
\hline 1 & $\mathrm{Cl}$ & -0.282373 & 0.476882 & -0.012451 \\
2 & $\mathrm{Si}$ & -0.564281 & 0.488128 & 2.062849 \\
3 & $\mathrm{~S}$ & 1.164340 & -0.346334 & 3.025559 \\
4 & $\mathrm{C}$ & 2.547505 & 0.536282 & 2.178674 \\
5 & $\mathrm{H}$ & 3.462619 & 0.224517 & 2.687704 \\
6 & $\mathrm{H}$ & 2.600204 & 0.251252 & 1.126203 \\
7 & $\mathrm{H}$ & 2.434429 & 1.618993 & 2.269569 \\
8 & $\mathrm{~S}$ & -0.930754 & 2.550158 & 2.514437 \\
9 & $\mathrm{C}$ & -0.990543 & 2.515383 & 4.361286 \\
10 & $\mathrm{H}$ & -1.051472 & 3.559308 & 4.678477 \\
11 & $\mathrm{H}$ & -1.873046 & 1.979070 & 4.715631 \\
12 & $\mathrm{H}$ & -0.085921 & 2.063668 & 4.774489 \\
13 & $\mathrm{~S}$ & -2.260901 & -0.675239 & 2.644076 \\
14 & $\mathrm{C}$ & -1.766126 & -2.393139 & 2.173550 \\
15 & $\mathrm{H}$ & -0.888403 & -2.720816 & 2.732934 \\
16 & $\mathrm{H}$ & -2.618008 & -3.028501 & 2.427573 \\
17 & $\mathrm{H}$ & -1.576261 & -2.459465 & 1.100073 \\
\hline
\end{tabular}

Total Energy of $(\mathrm{MeS})_{3} \mathrm{SiCl}[\mathrm{B} 3 \mathrm{LYP} / 6-31+\mathrm{G}(\mathrm{d})]$ : $\mathrm{E}(\mathrm{RB}+\mathrm{HF}-\mathrm{LYP})=-2064.14722839$ Hartree Corrected Zero Point Energy: -2064.025442 Hartree

Table 52. Standard orientation of (MeS) $)_{3}$ SiLi $[B 3 L Y P / 6-31+G(d)]$

\begin{tabular}{ccccc}
$\begin{array}{c}\text { Center } \\
\text { Number }\end{array}$ & $\begin{array}{c}\text { Atom } \\
\text { Type }\end{array}$ & \multicolumn{3}{c}{ Coordinates (Angstroms) } \\
\hline 1 & $\mathrm{Li}$ & -0.497324 & -0.500674 & -1.000950 \\
2 & $\mathrm{Si}$ & 0.097727 & 0.000254 & 1.347933 \\
3 & $\mathrm{~S}$ & 2.147949 & -0.780400 & 1.430906 \\
4 & $\mathrm{C}$ & 2.831239 & -0.315973 & 3.082499 \\
5 & $\mathrm{H}$ & 3.759529 & -0.877769 & 3.217683 \\
6 & $\mathrm{H}$ & 3.043029 & 0.754480 & 3.126886 \\
7 & $\mathrm{H}$ & 2.123097 & -0.585540 & 3.870348 \\
8 & $\mathrm{~S}$ & 0.357371 & 2.150302 & 1.785554 \\
9 & $\mathrm{C}$ & -1.383601 & 2.751449 & 1.726533 \\
10 & $\mathrm{H}$ & -1.353072 & 3.832888 & 1.885764
\end{tabular}




\begin{tabular}{lllll}
11 & $\mathrm{H}$ & -1.844913 & 2.554204 & 0.752208 \\
12 & $\mathrm{H}$ & -1.987390 & 2.290068 & 2.511486 \\
13 & $\mathrm{~S}$ & -1.017205 & -0.683532 & 3.127840 \\
14 & $\mathrm{C}$ & -1.489280 & -2.386911 & 2.612256 \\
15 & $\mathrm{H}$ & -0.605738 & -3.016711 & 2.475552 \\
16 & $\mathrm{H}$ & -2.078458 & -2.377503 & 1.688435 \\
17 & $\mathrm{H}$ & -2.102957 & -2.808630 & 3.413200 \\
\hline
\end{tabular}

Total Energy of (MeS) ${ }_{3}$ SiLi [B3LYP/6-31+G(d)]: E(RB+HF-LYP) $=-1611.41969747$ Hartree Corrected Zero Point Energy: -1611.299620 Hartree

Table 53. Shielding tensor for 4 [GIAO-HF/6-311+G(2d,p)//B3LYP/6-31+G(d)].

\begin{tabular}{cccc} 
Si & Isotropic $=364.8913$ & Anisotropy $=85.5672$ & \\
\hline & $X X=412.2617$ & $Y X=10.6611$ & $Z X=-35.4557$ \\
& $X Y=21.9135$ & $Y Y=380.4082$ & $Z Y=-0.7393$ \\
& $X Z=-10.3942$ & $Y Z=17.0835$ & $Z Z=302.0040$ \\
\hline Eigenvalues: & 295.9115 & 376.8263 & 421.9361
\end{tabular}

Table 54. Shielding tensor for 5 [GIAO-HF/6-311+G(2d,p)//B3LYP/6-31+G(d)].

\begin{tabular}{cccc} 
Si & Isotropic $=361.2612$ & Anisotropy $=78.1633$ & \\
\hline & $\mathrm{XX}=407.7541$ & $\mathrm{YX}=12.4860$ & $\mathrm{ZX}=-5.3376$ \\
& $\mathrm{XY}=29.2672$ & $\mathrm{YY}=333.0137$ & $\mathrm{ZY}=-32.8108$ \\
& $\mathrm{XZ}=11.3974$ & $\mathrm{YZ}=-17.2143$ & $\mathrm{ZZ}=343.0159$ \\
\hline Eigenvalues: & 309.1235 & 361.2902 & 413.3701
\end{tabular}

Table 55. Shielding tensor for 6 [GIAO-HF/6-311+G(2d,p)//B3LYP/6-31+G(d)].

\begin{tabular}{cccc} 
Si & Isotropic $=362.9025$ & Anisotropy $=102.8504$ & \\
\hline & $X X=415.2963$ & $Y X=-15.2893$ & $Z X=32.3100$ \\
& $X Y=-25.2802$ & $Y Y=397.7634$ & $Z Y=-9.4826$ \\
& $X Z=15.5734$ & $Y Z=6.4560$ & $Z Z=275.6478$ \\
\hline Eigenvalues: & 271.6297 & 385.6084 & 431.4694
\end{tabular}

Table 56. Shielding tensor for 4.3 THF [GIAO-HF/6-311+G(2d,p)//B3LYP/TZVP].

\begin{tabular}{cccc} 
Si & Isotropic $=362.1172$ & Anisotropy $=131.8445$ & \\
& $\mathrm{XX}=444.8564$ & $\mathrm{YX}=11.6061$ & $\mathrm{ZX}=-9.1695$ \\
& $\mathrm{XY}=-2.6013$ & $\mathrm{YY}=281.0304$ & $\mathrm{ZY}=-13.1250$ \\
$\mathrm{XZ}=-31.7789$ & $\mathrm{YZ}=-24.9894$ & $\mathrm{ZZ}=360.4648$ \\
\hline Eigenvalues: & 276.6948 & 359.6433 & 450.0135
\end{tabular}

Table 57. Shielding tensor for 5.3 THF [GIAO-HF/6-311+G(2d,p)//B3LYP/TZVP].

\begin{tabular}{cccc} 
Si & Isotropic $=349.7151$ & Anisotropy $=140.9840$ & \\
\hline & $X X=442.1454$ & $Y X=1.7715$ & $Z X=-13.5934$ \\
& $X Y=16.8298$ & $Y Y=288.0587$ & $Z Y=-25.4463$ \\
& $X Z=-6.0456$ & $Y Z=-16.1542$ & $Z Z=318.9412$ \\
\hline Eigenvalues: & 277.5007 & 327.9402 & 443.7044
\end{tabular}

Table 58. Shielding tensor for 6.3 THF [GIAO-HF/6-311+G(2d,p)//B3LYP/TZVP]. 


\begin{tabular}{lccc}
\hline & $X X=437.7883$ & $Y X=-12.8697$ & $Z X=44.4906$ \\
& $X Y=-21.5062$ & $Y Y=402.9170$ & $Z Y=-25.1392$ \\
& $X Z=9.1212$ & $Y Z=-1.2786$ & $Z Z=259.5641$ \\
\hline Eigenvalues: & 254.8557 & 395.8967 & 449.5169
\end{tabular}

Table 59. Shielding tensor for $\mathrm{Me}_{3} \mathrm{SiH}[\mathrm{GIAO}-\mathrm{HF} / 6-311+\mathrm{G}(2 \mathrm{~d}, \mathrm{p}) / / \mathrm{B} 3 \mathrm{LYP} / 6-31+\mathrm{G}(\mathrm{d})]$.

\begin{tabular}{cccc} 
Si & Isotropic $=400.5132$ & Anisotropy $=20.3420$ & \\
\hline & $X X=414.0742$ & $Y X=-0.0260$ & $Z X=0.0363$ \\
& $X Y=0.0193$ & $Y Y=413.9877$ & $Z Y=-0.1229$ \\
& $X Z=0.1306$ & $Y Z=0.2993$ & $Z Z=373.4776$ \\
\hline Eigenvalues: & 373.4772 & 413.9878 & 414.0745
\end{tabular}

Table 60. Shielding tensor for $\mathrm{Me}_{3} \mathrm{SiCl}[\mathrm{GIAO}-\mathrm{HF} / 6-311+\mathrm{G}(2 \mathrm{~d}, \mathrm{p}) / / \mathrm{B} 3 \mathrm{LYP} / 6-31+\mathrm{G}(\mathrm{d})]$.

\begin{tabular}{cccc} 
Si & Isotropic $=355.0845$ & Anisotropy $=4.0440$ & \\
\hline & $X X=357.7803$ & $Y X=0.0166$ & $Z X=0.0488$ \\
& $X Y=-0.0546$ & $Y Y=353.7915$ & $Z Y=-0.0529$ \\
& $X Z=-0.0070$ & $Y Z=-0.0594$ & $Z Z=353.6817$ \\
\hline Eigenvalues: & 353.6580 & 353.8150 & 357.7805
\end{tabular}

Table 61. Shielding tensor for Me ${ }_{3}$ SiLi [GIAO-HF/6-311+G(2d,p)//B3LYP/6-31+G(d)].

\begin{tabular}{cccc} 
Si & Isotropic $=400.1034$ & Anisotropy $=19.2992$ & \\
\hline & $\mathrm{XX}=393.6518$ & $\mathrm{YX}=-0.0829$ & $\mathrm{ZX}=-0.2493$ \\
& $\mathrm{XY}=0.0670$ & $\mathrm{YY}=393.6900$ & $\mathrm{ZY}=0.0755$ \\
& $\mathrm{XZ}=-0.0240$ & $\mathrm{YZ}=0.0545$ & $\mathrm{ZZ}=412.9683$ \\
\hline Eigenvalues: & 393.6494 & 393.6911 & 412.9695
\end{tabular}

Table 62. Shielding tensor for $\mathrm{Me}_{4} \mathrm{Si}[\mathrm{GIAO}-\mathrm{HF} / 6-311+\mathrm{G}(2 \mathrm{~d}, \mathrm{p}) / / \mathrm{B} 3 \mathrm{LYP} / 6-31+\mathrm{G}(\mathrm{d})]$.

\begin{tabular}{cccc} 
Si & Isotropic $=385.8591$ & Anisotropy $=0.2188$ & \\
\hline & $X X=385.8553$ & $Y X=-0.5095$ & $Z X=0.1424$ \\
& $X Y=0.4205$ & $Y Y=385.9917$ & $Z Y=-0.4635$ \\
& $X Z=0.0481$ & $Y Z=0.5116$ & $Z Z=385.7304$ \\
\hline Eigenvalues: & 385.6733 & 385.8991 & 386.0050
\end{tabular}

Table 63. Shielding tensor for $\mathrm{Si}$ in (MeO)Me ${ }_{2} \mathrm{SiH}[\mathrm{GIAO}-\mathrm{HF} / 6-311+\mathrm{G}(2 \mathrm{~d}, \mathrm{p}) / / \mathrm{B} 3 \mathrm{LYP} / 6-31+\mathrm{G}(\mathrm{d})]$.

\begin{tabular}{cccc} 
Si & Isotropic $=382.6067$ & Anisotropy $=53.6363$ & \\
\hline & $X X=414.4888$ & $Y X=-0.8612$ & $Z X=-22.7096$ \\
& $X Y=-0.0296$ & $Y Y=388.4254$ & $Z Y=7.0292$ \\
& $X Z=-10.3340$ & $Y Z=8.1828$ & $Z Z=344.9059$ \\
\hline Eigenvalues: & 340.0721 & 389.3837 & 418.3642
\end{tabular}

Table 64. Shielding tensor for $\mathrm{Si}$ in (MeO)Me ${ }_{2} \mathrm{SiCl}[\mathrm{GIAO}-\mathrm{HF} / 6-311+\mathrm{G}(2 \mathrm{~d}, \mathrm{p}) / / \mathrm{B} 3 \mathrm{~L} Y \mathrm{P} / 6-31+\mathrm{G}(\mathrm{d})]$. Si $\quad$ Isotropic $=370.0403 \quad$ Anisotropy $=57.0978$

\begin{tabular}{lccc}
\hline & $X X=373.7256$ & $Y X=6.5067$ & $Z X=8.6945$ \\
& $X Y=19.3338$ & $Y Y=338.4250$ & $Z Y=9.6172$ \\
& $X Z=19.5045$ & $Y Z=13.3813$ & $Z Z=397.9704$ \\
\hline Eigenvalues: & 333.4623 & 368.5531 & 408.1055
\end{tabular}


Table 65. Shielding tensor for Si in (MeO)Me ${ }_{2}$ SiLi [GIAO-HF/6-311+G(2d,p)//B3LYP/6-31+G(d)].

\begin{tabular}{cccc} 
Si & Isotropic $=346.5670$ & Anisotropy $=82.4505$ & \\
\hline & $\mathrm{XX}=385.0833$ & $\mathrm{YX}=29.3335$ & $\mathrm{ZX}=16.3225$ \\
& $\mathrm{XY}=41.8436$ & $\mathrm{YY}=271.8343$ & $\mathrm{ZY}=-3.0513$ \\
& $\mathrm{XZ}=7.4265$ & $\mathrm{YZ}=-1.9993$ & $\mathrm{ZZ}=382.7834$ \\
\hline Eigenvalues: & 261.3085 & 376.8585 & 401.5339
\end{tabular}

Table 66. Shielding tensor for $\mathrm{Si}$ in (MeO)Me ${ }_{2} \mathrm{SiMe}[\mathrm{GIAO}-\mathrm{HF} / 6-311+\mathrm{G}(2 \mathrm{~d}, \mathrm{p}) / / \mathrm{B} 3 \mathrm{LYP} / 6-31+\mathrm{G}(\mathrm{d})]$.

\begin{tabular}{cccc} 
Si & Isotropic $=371.5865$ & Anisotropy $=29.6307$ & \\
\hline & $\mathrm{XX}=390.2990$ & $\mathrm{YX}=2.0578$ & $\mathrm{ZX}=8.9021$ \\
& $\mathrm{XY}=-1.0759$ & $\mathrm{YY}=359.0284$ & $\mathrm{ZY}=3.2598$ \\
& $\mathrm{XZ}=1.2723$ & $\mathrm{YZ}=3.5811$ & $\mathrm{ZZ}=365.4322$ \\
\hline Eigenvalues: & 357.4634 & 365.9558 & 391.3403
\end{tabular}

Table 67. Shielding tensor for $\mathrm{Si}$ in $\left(\mathrm{Me}_{2} \mathrm{~N}\right) \mathrm{Me}_{2} \mathrm{SiH}$ [GIAO-HF/6-311+G(2d,p)//B3LYP/6-31+G(d)].

\begin{tabular}{cccc} 
Si & Isotropic $=393.2873$ & Anisotropy $=58.3882$ & \\
\hline & $\mathrm{XX}=422.6095$ & $\mathrm{YX}=0.0000$ & $\mathrm{ZX}=30.9572$ \\
& $\mathrm{XY}=0.0000$ & $\mathrm{YY}=400.3888$ & $\mathrm{ZY}=0.0000$ \\
& $\mathrm{XZ}=22.8423$ & $\mathrm{YZ}=0.0000$ & $\mathrm{ZZ}=356.8635$ \\
\hline Eigenvalues: & 347.2603 & 400.3888 & 432.2128
\end{tabular}

Table 68. Shielding tensor for $\mathrm{Si}$ in $\left(\mathrm{Me}_{2} \mathrm{~N}\right) \mathrm{Me}_{2} \mathrm{SiCl}[\mathrm{GIAO}-\mathrm{HF} / 6-311+\mathrm{G}(2 \mathrm{~d}, \mathrm{p}) / / \mathrm{B} 3 \mathrm{LYP} / 6-31+\mathrm{G}(\mathrm{d})]$.

\begin{tabular}{cccc} 
Si & Isotropic $=369.5036$ & Anisotropy $=29.7167$ & \\
\hline & $X X=388.2306$ & $Y X=5.7907$ & $Z X=12.9042$ \\
$X Y=0.6046$ & $Y Y=376.9418$ & $Z Y=-17.6693$ \\
& $X Z=0.8625$ & $Y Z=-17.7645$ & $Z Z=343.3384$ \\
\hline
\end{tabular}

Eigenvalues: $\quad 334.6490 \quad 384.5471 \quad 389.3147$

Table 69. Shielding tensor for Si in $\left(\mathrm{Me}_{2} \mathrm{~N}\right) \mathrm{Me}_{2} \mathrm{SiLi}[\mathrm{GIAO}-\mathrm{HF} / 6-311+\mathrm{G}(2 \mathrm{~d}, \mathrm{p}) / / \mathrm{B} 3 \mathrm{LYP} / 6-31+\mathrm{G}(\mathrm{d})]$.

\begin{tabular}{cccc} 
Si & Isotropic $=362.4871$ & Anisotropy $=63.9211$ & \\
\hline & $\mathrm{XX}=399.7999$ & $\mathrm{YX}=0.0000$ & $\mathrm{ZX}=10.1918$ \\
& $\mathrm{XY}=0.0000$ & $\mathrm{YY}=298.4311$ & $\mathrm{ZY}=0.0000$ \\
$\mathrm{XZ}=8.1534$ & $\mathrm{YZ}=0.0000$ & $\mathrm{ZZ}=389.2303$ \\
\hline Eigenvalues: & 298.4311 & 383.9290 & 405.1012
\end{tabular}

Table 70. Shielding tensor for Si in $\left(\mathrm{Me}_{2} \mathrm{~N}\right) \mathrm{Me}_{2} \mathrm{SiMe}$ [GIAO-HF/6-311+G(2d,p)//B3LYP/6-31+G(d)].

\begin{tabular}{cccc} 
Si & Isotropic $=380.2253$ & Anisotropy $=36.7478$ & \\
\hline & $\mathrm{XX}=390.5399$ & $\mathrm{YX}=12.1714$ & $\mathrm{ZX}=12.2522$ \\
& $\mathrm{XY}=14.8891$ & $\mathrm{YY}=370.5405$ & $\mathrm{ZY}=4.4094$ \\
& $\mathrm{XZ}=14.1938$ & $\mathrm{YZ}=3.3701$ & $\mathrm{ZZ}=379.5954$ \\
\hline Eigenvalues: & 363.1589 & 372.7931 & 404.7238
\end{tabular}

Table 71. Shielding tensor for $\mathrm{Si}$ in (MeS)Me ${ }_{2} \mathrm{SiH}$ [GIAO-HF/6-311+G(2d,p)//B3LYP/6-31+G(d)].

\begin{tabular}{cccc} 
Si & Isotropic $=383.3101$ & Anisotropy $=40.1262$ & \\
\hline$X X=408.0430$ & $Y X=3.1157$ & $Z X=-13.3018$ \\
$X Y=-5.7056$ & $Y Y=383.9161$ & $Z Y=-16.8009$ \\
$X Z=-6.5889$ & $Y Z=-13.6014$ & $Z Z=357.9710$
\end{tabular}




\begin{tabular}{llll}
\hline Eigenvalues: & 349.3912 & 390.4781 & 410.0609
\end{tabular}

Table 72. Shielding tensor for $\mathrm{Si}$ in (MeS)Me $\mathrm{SiCl}_{2}$ [GIAO-HF/6-311+G(2d,p)//B3LYP/6-31+G(d)].

\begin{tabular}{cccc} 
Si & Isotropic $=348.9146$ & Anisotropy $=19.7493$ & \\
& $\mathrm{XX}=352.4305$ & $\mathrm{YX}=4.8323$ & $\mathrm{ZX}=1.5817$ \\
& $\mathrm{XY}=8.1490$ & $\mathrm{YY}=340.0805$ & $\mathrm{ZY}=-13.7288$ \\
& $\mathrm{XZ}=8.1103$ & $\mathrm{YZ}=-12.3331$ & $\mathrm{ZZ}=354.2328$ \\
\hline Eigenvalues: & 329.4960 & 355.1670 & 362.0808
\end{tabular}

Table 73. Shielding tensor for Si in (MeS)Me ${ }_{2}$ SiLi [GIAO-HF/6-311+G(2d,p)//B3LYP/6-31+G(d)].

\begin{tabular}{cccc} 
Si & Isotropic $=366.0408$ & Anisotropy $=48.3704$ & \\
& $\mathrm{XX}=391.1863$ & $\mathrm{YX}=-1.0346$ & $\mathrm{ZX}=7.9448$ \\
& $\mathrm{XY}=38.2293$ & $\mathrm{YY}=310.7230$ & $\mathrm{ZY}=-3.5988$ \\
& $\mathrm{XZ}=-4.6092$ & $\mathrm{YZ}=11.0375$ & $\mathrm{ZZ}=396.2133$ \\
\hline Eigenvalues: & 306.5129 & 393.3218 & 398.2878
\end{tabular}

Table 74. Shielding tensor for $\mathrm{Si}$ in (MeS)Me 2 SiMe [GIAO-HF/6-311+G(2d,p)//B3LYP/6-31+G(d)].

\begin{tabular}{cccc} 
Si & Isotropic $=370.1362$ & Anisotropy $=16.6867$ & \\
\hline & $\mathrm{XX}=379.9888$ & $\mathrm{YX}=0.8563$ & $\mathrm{ZX}=0.0127$ \\
& $\mathrm{XY}=1.8376$ & $\mathrm{YY}=349.1594$ & $\mathrm{ZY}=0.0260$ \\
& $\mathrm{XZ}=0.0243$ & $\mathrm{YZ}=0.0037$ & $\mathrm{ZZ}=381.2603$ \\
\hline Eigenvalues: & 349.1007 & 380.0472 & 381.2606
\end{tabular}

Table 75. Shielding tensor for $\mathrm{Si}$ in $(\mathrm{MeO})_{2} \mathrm{MeSiH}[\mathrm{GIAO}-\mathrm{HF} / 6-311+\mathrm{G}(2 \mathrm{~d}, \mathrm{p}) / / \mathrm{B} 3 \mathrm{LYP} / 6-31+\mathrm{G}(\mathrm{d})]$.

\begin{tabular}{cccc} 
Si & Isotropic $=401.7629$ & Anisotropy $=47.7187$ & \\
\hline & $\mathrm{XX}=404.0912$ & $\mathrm{YX}=-16.6883$ & $\mathrm{ZX}=1.1826$ \\
& $\mathrm{XY}=-17.3538$ & $\mathrm{YY}=406.4783$ & $\mathrm{ZY}=-20.3197$ \\
& $\mathrm{XZ}=15.8932$ & $\mathrm{YZ}=-19.9577$ & $\mathrm{ZZ}=394.7191$ \\
\hline Eigenvalues: & 378.9437 & 392.7695 & 433.5754
\end{tabular}

Table 76. Shielding tensor for Si in (MeO) ${ }_{2} \mathrm{MeSiCl}[\mathrm{GIAO}-\mathrm{HF} / 6-311+\mathrm{G}(2 \mathrm{~d}, \mathrm{p}) / / \mathrm{B} 3 \mathrm{LYP} / 6-31+\mathrm{G}(\mathrm{d})]$.

\begin{tabular}{cccc} 
Si & Isotropic $=405.2334$ & Anisotropy $=54.6961$ & \\
\hline & $\mathrm{XX}=384.8454$ & $\mathrm{YX}=-8.4477$ & $\mathrm{ZX}=-7.7800$ \\
& $\mathrm{XY}=-3.8849$ & $\mathrm{YY}=409.4060$ & $\mathrm{ZY}=-18.8849$ \\
& $\mathrm{XZ}=8.0113$ & $\mathrm{YZ}=-31.6987$ & $\mathrm{ZZ}=421.4487$ \\
\hline Eigenvalues: & 381.6629 & 392.3398 & 441.6974
\end{tabular}

Table 77. Shielding tensor for Si in (MeO) ${ }_{2} \mathrm{MeSiLi}$ [GIAO-HF/6-311+G(2d,p)//B3LYP/6-31+G(d)].

\begin{tabular}{cccc} 
Si & Isotropic $=350.8385$ & Anisotropy $=137.4416$ & \\
\hline & $\mathrm{XX}=345.2640$ & $\mathrm{YX}=2.8161$ & $\mathrm{ZX}=39.0517$ \\
& $\mathrm{XY}=22.7525$ & $\mathrm{YY}=291.4601$ & $\mathrm{ZY}=23.4214$ \\
& $\mathrm{XZ}=50.9649$ & $\mathrm{YZ}=23.7711$ & $\mathrm{ZZ}=415.7915$ \\
\hline Eigenvalues: & 286.6638 & 323.3856 & 442.4662
\end{tabular}

Table 78. Shielding tensor for Si in (MeO) ${ }_{2} \mathrm{MeSiMe}[\mathrm{GIAO}-\mathrm{HF} / 6-311+\mathrm{G}(2 \mathrm{~d}, \mathrm{p}) / / \mathrm{B} 3 \mathrm{LYP} / 6-31+\mathrm{G}(\mathrm{d})]$. Si $\quad$ Isotropic $=388.7870 \quad$ Anisotropy $=53.2009$ 


\begin{tabular}{lccc} 
& $X X=383.3079$ & $Y X=-0.0003$ & $Z X=17.0912$ \\
& $X Y=0.0001$ & $Y Y=374.6570$ & $Z Y=-0.0002$ \\
& $X Z=33.8729$ & $Y Z=-0.0001$ & $Z Z=408.3960$ \\
\hline Eigenvalues: & 367.4497 & 374.6570 & 424.2542
\end{tabular}

Table 79. Shielding tensor for $\mathrm{Si}$ in $\left(\mathrm{Me}_{2} \mathrm{~N}\right)_{2} \mathrm{MeSiH}[\mathrm{GIAO}-\mathrm{HF} / 6-311+\mathrm{G}(2 \mathrm{~d}, \mathrm{p}) / / \mathrm{B} 3 \mathrm{LYP} / 6-31+\mathrm{G}(\mathrm{d})]$.

\begin{tabular}{cccc} 
Si & Isotropic $=394.3760$ & Anisotropy $=40.5089$ & \\
\hline & $\mathrm{XX}=392.9126$ & $\mathrm{YX}=12.9568$ & $\mathrm{ZX}=11.1321$ \\
& $\mathrm{XY}=13.9590$ & $\mathrm{YY}=410.9973$ & $\mathrm{ZY}=13.2006$ \\
& $\mathrm{XZ}=5.3816$ & $\mathrm{YZ}=4.2903$ & $\mathrm{ZZ}=379.2181$ \\
\hline Eigenvalues: & 375.1399 & 386.6061 & 421.3820
\end{tabular}

Table 80. Shielding tensor for $\mathrm{Si}$ in $\left(\mathrm{Me}_{2} \mathrm{~N}\right)_{2} \mathrm{MeSiCl}[\mathrm{GIAO}-\mathrm{HF} / 6-311+\mathrm{G}(2 \mathrm{~d}, \mathrm{p}) / / \mathrm{B} 3 \mathrm{LYP} / 6-31+\mathrm{G}(\mathrm{d})]$.

\begin{tabular}{cccc} 
Si & Isotropic $=389.1815$ & Anisotropy $=37.5170$ & \\
\hline & $\mathrm{XX}=406.8512$ & $\mathrm{YX}=12.9100$ & $\mathrm{ZX}=3.5261$ \\
& $\mathrm{XY}=2.6228$ & $\mathrm{YY}=405.0131$ & $\mathrm{ZY}=-0.3026$ \\
& $\mathrm{XZ}=7.5388$ & $\mathrm{YZ}=3.0731$ & $\mathrm{ZZ}=355.6802$ \\
\hline Eigenvalues: & 355.0826 & 398.2690 & 414.1928
\end{tabular}

Table 81. Shielding tensor for Si in $\left(\mathrm{Me}_{2} \mathrm{~N}\right)_{2} \mathrm{MeSiLi}[\mathrm{GIAO}-\mathrm{HF} / 6-311+\mathrm{G}(2 \mathrm{~d}, \mathrm{p}) / / \mathrm{B} 3 \mathrm{LYP} / 6-31+\mathrm{G}(\mathrm{d})]$.

\begin{tabular}{cccc} 
Si & Isotropic $=352.7918$ & Anisotropy $=79.6951$ & \\
\hline & $\mathrm{XX}=354.7447$ & $\mathrm{YX}=-22.6022$ & $\mathrm{ZX}=-14.9687$ \\
& $\mathrm{XY}=-36.1742$ & $\mathrm{YY}=303.6889$ & $\mathrm{ZY}=7.8582$ \\
& $\mathrm{XZ}=-11.3277$ & $\mathrm{YZ}=6.8471$ & $\mathrm{ZZ}=399.9419$ \\
\hline Eigenvalues: & 290.2751 & 362.1786 & 405.9219
\end{tabular}

Table 82. Shielding tensor for Si in $\left(\mathrm{Me}_{2} \mathrm{~N}\right)_{2} \mathrm{MeSiMe}[\mathrm{GIAO}-\mathrm{HF} / 6-311+\mathrm{G}(2 \mathrm{~d}, \mathrm{p}) / / \mathrm{B} 3 \mathrm{LYP} / 6-31+\mathrm{G}(\mathrm{d})]$.

\begin{tabular}{cccc} 
Si & Isotropic $=388.0573$ & Anisotropy $=25.0932$ & \\
\hline & $X X=401.1782$ & $Y X=-2.4734$ & $Z X=-6.1347$ \\
& $X Y=0.7197$ & $Y Y=380.8016$ & $Z Y=2.1326$ \\
& $X Z=-11.4496$ & $Y Z=4.1323$ & $Z Z=382.1921$ \\
\hline Eigenvalues: & 376.9368 & 382.4490 & 404.7861
\end{tabular}

Table 83. Shielding tensor for $\mathrm{Si}$ in (MeS) ${ }_{2} \mathrm{MeSiH}[\mathrm{GIAO}-\mathrm{HF} / 6-311+\mathrm{G}(2 \mathrm{~d}, \mathrm{p}) / / \mathrm{B} 3 \mathrm{LYP} / 6-31+\mathrm{G}(\mathrm{d})]$.

\begin{tabular}{cccc} 
Si & Isotropic $=373.4969$ & Anisotropy $=68.3787$ & \\
\hline & $X X=400.8291$ & $Y X=16.9883$ & $Z X=-6.1301$ \\
$X Y=11.2393$ & $Y Y=401.1179$ & $Z Y=-25.7576$ \\
& $X Z=-2.8048$ & $Y Z=-20.2975$ & $Z Z=318.5438$ \\
\hline
\end{tabular}

Eigenvalues: $\quad 312.5493 \quad 388.8588 \quad 419.0827$

Table 84. Shielding tensor for $\mathrm{Si}$ in (MeS) $)_{2} \mathrm{MeSiCl}$ [GIAO-HF/6-311+G(2d,p)//B3LYP/6-31+G(d)].

\begin{tabular}{cccc} 
Si & Isotropic $=344.5575$ & Anisotropy $=49.7179$ & \\
& $\mathrm{XX}=360.6323$ & $\mathrm{YX}=-6.2988$ & $\mathrm{ZX}=-5.3868$ \\
& $\mathrm{XY}=-7.3691$ & $\mathrm{YY}=360.5875$ & $\mathrm{ZY}=-25.8974$ \\
& $\mathrm{XZ}=0.7125$ & $\mathrm{YZ}=-37.0855$ & $\mathrm{ZZ}=312.4527$ \\
\hline Eigenvalues: & 296.4736 & 359.4961 & 377.7028
\end{tabular}


Table 85. Shielding tensor for Si in (MeS) ${ }_{2} \mathrm{MeSiLi}[\mathrm{GIAO}-\mathrm{HF} / 6-311+\mathrm{G}(2 \mathrm{~d}, \mathrm{p}) / / \mathrm{B} 3 \mathrm{LYP} / 6-31+\mathrm{G}(\mathrm{d})]$.

\begin{tabular}{cccc} 
Si & Isotropic $=351.2563$ & Anisotropy $=43.8700$ & \\
\hline & $\mathrm{XX}=363.4211$ & $\mathrm{YX}=-21.3454$ & $\mathrm{ZX}=17.3880$ \\
& $\mathrm{XY}=4.2974$ & $\mathrm{YY}=342.8683$ & $\mathrm{ZY}=31.6023$ \\
& $\mathrm{XZ}=11.2983$ & $\mathrm{YZ}=35.6400$ & $\mathrm{ZZ}=347.4795$ \\
\hline Eigenvalues: & 306.9085 & 366.3575 & 380.5030
\end{tabular}

Table 86. Shielding tensor for $\mathrm{Si}$ in (MeS) ${ }_{2} \mathrm{MeSiMe}$ [GIAO-HF/6-311+G(2d,p)//B3LYP/6-31+G(d)].

\begin{tabular}{cccc} 
Si & Isotropic $=356.4463$ & Anisotropy $=33.3461$ & \\
\hline & $\mathrm{XX}=372.7199$ & $\mathrm{YX}=-0.0112$ & $\mathrm{ZX}=6.4487$ \\
& $\mathrm{XY}=-0.0056$ & $\mathrm{YY}=378.6770$ & $\mathrm{ZY}=-0.0151$ \\
& $\mathrm{XZ}=10.5951$ & $\mathrm{YZ}=0.0100$ & $\mathrm{ZZ}=317.9420$ \\
\hline Eigenvalues: & 316.6469 & 374.0150 & 378.6771
\end{tabular}

Table 87. Shielding tensor for $\mathrm{Si}$ in (MeO) ${ }_{3} \mathrm{SiCl}[\mathrm{GIAO}-\mathrm{HF} / 6-311+\mathrm{G}(2 \mathrm{~d}, \mathrm{p}) / / \mathrm{B} 3 \mathrm{LYP} / 6-31+\mathrm{G}(\mathrm{d})]$.

\begin{tabular}{cccc} 
Si & Isotropic $=448.1875$ & Anisotropy $=18.6428$ & \\
\hline & $X X=447.4452$ & $Y X=-10.4129$ & $Z X=4.0130$ \\
& $X Y=17.7449$ & $Y Y=458.4704$ & $Z Y=-5.3961$ \\
& $X Z=-5.2544$ & $Y Z=15.6789$ & $Z Z=438.6469$ \\
\hline Eigenvalues: & 437.1708 & 446.7757 & 460.6160
\end{tabular}

Table 88. Shielding tensor for Si in (MeO) ${ }_{3}$ SiLi [GIAO-HF/6-311+G(2d,p)//B3LYP/6-31+G(d)].

\begin{tabular}{cccc} 
Si & Isotropic $=394.4748$ & Anisotropy $=138.5827$ & \\
\hline & $X X=350.9189$ & $Y X=12.8092$ & $Z X=21.3528$ \\
& $X Y=10.4724$ & $Y Y=360.6510$ & $Z Y=24.1242$ \\
& $X Z=44.1672$ & $Y Z=29.9586$ & $Z Z=471.8544$ \\
\hline Eigenvalues: & 341.0263 & 355.5348 & 486.8632
\end{tabular}

Table 89. Shielding tensor for $\mathrm{Si}$ in $\left(\mathrm{Me}_{2} \mathrm{~N}\right)_{3} \mathrm{SiCl}[\mathrm{GIAO}-\mathrm{HF} / 6-311+\mathrm{G}(2 \mathrm{~d}, \mathrm{p}) / / \mathrm{B} 3 \mathrm{LYP} / 6-31+\mathrm{G}(\mathrm{d})]$.

\begin{tabular}{cccc} 
Si & Isotropic $=410.1845$ & Anisotropy $=31.2251$ & \\
\hline & $\mathrm{XX}=430.8496$ & $\mathrm{YX}=4.5602$ & $\mathrm{ZX}=0.9335$ \\
& $\mathrm{XY}=-6.8506$ & $\mathrm{YY}=422.1969$ & $\mathrm{ZY}=11.4066$ \\
& $\mathrm{XZ}=1.6013$ & $\mathrm{YZ}=12.9222$ & $\mathrm{ZZ}=377.5070$ \\
\hline Eigenvalues: & 374.3701 & 425.1822 & 431.0013
\end{tabular}

Table 90. Shielding tensor for Si in $\left(\mathrm{Me}_{2} \mathrm{~N}\right)_{3} \mathrm{SiLi}[\mathrm{GIAO}-\mathrm{HF} / 6-311+\mathrm{G}(2 \mathrm{~d}, \mathrm{p}) / / \mathrm{B} 3 \mathrm{LYP} / 6-31+\mathrm{G}(\mathrm{d})]$.

\begin{tabular}{cccc} 
Si & Isotropic $=363.2523$ & Anisotropy $=97.9330$ & \\
\hline & $X X=328.3358$ & $Y X=8.2523$ & $Z X=11.6915$ \\
& $X Y=5.4705$ & $Y Y=341.6274$ & $Z Y=27.8751$ \\
& $X Z=11.4229$ & $Y Z=21.1753$ & $Z Z=419.7936$ \\
\hline Eigenvalues: & 325.4087 & 335.8072 & 428.5409
\end{tabular}

Table 91. Shielding tensor for Si in (MeS) ${ }_{3} \mathrm{SiCl}[\mathrm{GIAO}-\mathrm{HF} / 6-311+G(2 d, p) / / \mathrm{B} 3 \mathrm{LYP} / 6-31+\mathrm{G}(\mathrm{d})]$.

\begin{tabular}{cccc} 
Si & Isotropic $=341.7796$ & Anisotropy $=59.0246$ & \\
\hline$X X=362.4364$ & $Y X=-0.2603$ & $Z X=22.1065$ \\
& $X Y=19.7721$ & $Y Y=340.5436$ & $Z Y=24.9483$ \\
& $X Z=17.2482$ & $Y Z=28.0187$ & $Z Z=322.3588$
\end{tabular}


Table 92. Shielding tensor for Si in (MeS) ${ }_{3}$ SiLi [GIAO-HF/6-311+G(2d,p)//B3LYP/6-31+G(d)].

\begin{tabular}{cccc} 
Si & Isotropic $=335.3773$ & Anisotropy $=75.4673$ & \\
\hline & $X X=317.4748$ & $Y X=36.9313$ & $Z X=13.2098$ \\
& $X Y=-9.0057$ & $Y Y=318.8805$ & $Z Y=-18.2612$ \\
& $X Z=36.5232$ & $Y Z=-33.7704$ & $Z Z=369.7767$ \\
\hline Eigenvalues: & 288.3202 & 332.1230 & 385.6889
\end{tabular}

Table 93. Shielding tensor for Si in 4.3 THF in principal axes [IGLO-BP86/mixed basis//B3LYP/TZVP].

\begin{tabular}{c|ccc} 
& $\sigma_{1}$ & $\sigma_{2}$ & $\sigma_{3}$ \\
\hline$\sigma_{\mathrm{d}}$ & 829.4 & 829.7 & 827.4 \\
$\sigma_{\mathrm{p} 0}$ & 444.7 & 478.1 & 403.2 \\
$\sigma_{\mathrm{p} 1}$ & -1052.4 & -994.2 & -851.8 \\
$\sigma$ & 221.7 & 313.5 & 378.7 \\
\hline prin. axes & & & \\
\hline $\mathrm{x}$ & -0.0293 & 0.9689 & 0.2456 \\
$\mathrm{y}$ & $0.2357-$ & 0.2321 & 0.9437 \\
$\mathrm{z}$ & 0.9714 & 0.0855 & -0.2215
\end{tabular}

Table 94. Shielding tensor for Si in 5.3 THF in principal axes [IGLO-BP86/mixed basis//B3LYP/TZVP].

\begin{tabular}{c|ccc} 
& $\sigma_{1}$ & $\sigma_{2}$ & $\sigma_{3}$ \\
\hline$\sigma_{\mathrm{d}}$ & 827.0 & 829.5 & 826.3 \\
$\sigma_{\mathrm{p} 0}$ & 415.6 & 467.7 & 394.4 \\
$\sigma_{\mathrm{p} 1}$ & -1011.6 & -1017.6 & -849.9 \\
$\sigma$ & 231.0 & 279.6 & 370.8 \\
\hline prin. axes & & & \\
\hline $\mathrm{x}$ & 0.0378 & 0.8736 & 0.4851 \\
$\mathrm{y}$ & 0.1412 & -0.4852 & 0.8629 \\
$\mathrm{z}$ & 0.9893 & 0.0359 & -0.1417
\end{tabular}

Table 95. Shielding tensor for Si in 6.3 THF in principal axes [IGLO-BP86/mixed basis//B3LYP/TZVP

\begin{tabular}{c|ccc} 
& $\sigma_{1}$ & $\sigma_{2}$ & $\sigma_{3}$ \\
\hline$\sigma_{\mathrm{d}}$ & 829.1 & 831.3 & 828.4 \\
$\sigma_{\mathrm{p} 0}$ & 450.6 & 504.0 & 412.1 \\
$\sigma_{\mathrm{p} 1}$ & -1067.2 & -983.0 & -859.1 \\
$\sigma$ & 212.5 & 352.4 & 381.4 \\
\hline prin. axes & & & \\
\hline $\mathrm{x}$ & -0.2158 & 0.0340 & 0.9758 \\
$\mathrm{y}$ & 0.2278 & 0.9736 & 0.0164 \\
$\mathrm{z}$ & 0.9495 & -0.2258 & 0.2179
\end{tabular}

Table 96. Shielding tensor for $\mathrm{Me}_{3} \mathrm{SiH}$ in principal axes [IGLO-BP86/BIII/B3LYP/6-31+G(d)].

\begin{tabular}{c|ccc} 
& $\sigma_{1}$ & $\sigma_{2}$ & $\sigma_{3}$ \\
\hline$\sigma_{\mathrm{d}}$ & 823.6 & 828.6 & 828.6 \\
$\sigma_{\mathrm{p} 0}$ & 399.6 & 423.1 & 423.7 \\
$\sigma_{\mathrm{p} 1}$ & -905.5 & -885.7 & -886.3 \\
$\sigma$ & 317.7 & 366.0 & 366.1 \\
\hline prin. axes & & & \\
\hline $\mathrm{x}$ & -0.0012 & -0.0022 & 1.0000
\end{tabular}




$$
\begin{array}{l|ccc}
\mathrm{y} & -0.0394 & -0.9992 & -0.0022 \\
\mathrm{z} & 0.9992 & -0.0394 & 0.0011
\end{array}
$$

Table 97. Shielding tensor for $\mathrm{Si}$ in $\mathrm{Me}_{3} \mathrm{SiCl}$ in principal axes [IGLO-BP86/BIII//B3LYP/6-31+G(d)].

\begin{tabular}{c|ccc} 
& $\sigma_{1}$ & $\sigma_{2}$ & $\sigma_{3}$ \\
\hline$\sigma_{\mathrm{d}}$ & 827.0 & 827.0 & 827.0 \\
$\sigma_{\mathrm{p} 0}$ & 389.2 & 386.3 & 390.7 \\
$\sigma_{\mathrm{p} 1}$ & -934.3 & -931.3 & -904.3 \\
$\sigma$ & 281.8 & 282.0 & 313.5 \\
\hline prin. axes & & & \\
\hline $\mathrm{x}$ & -0.0011 & 0.4482 & 0.8939 \\
$\mathrm{y}$ & -0.0012 & -0.8939 & 0.4482 \\
$\mathrm{z}$ & 1.0000 & -0.0006 & 0.0016
\end{tabular}

Table 98.Shielding tensor for Si in $\mathrm{Me}_{3} \mathrm{SiLi}$ in principal axes [IGLO-BP86/BIII//B3LYP/6-31+G(d)].

\begin{tabular}{c|ccc} 
& $\sigma_{1}$ & $\sigma_{2}$ & $\sigma_{3}$ \\
\hline$\sigma_{\mathrm{d}}$ & 829.1 & 829.1 & 827.9 \\
$\sigma_{\mathrm{p} 0}$ & 438.5 & 438.5 & 413.3 \\
$\sigma_{\mathrm{p} 1}$ & -932.5 & -932.5 & -886.1 \\
$\sigma$ & 335.0 & 335.1 & 355.1 \\
\hline prin. axes & & & \\
\hline $\mathrm{x}$ & 0.5643 & 0.8256 & 0.0012 \\
$\mathrm{y}$ & 0.8255 & -0.5643 & 0.0096 \\
$\mathrm{z}$ & -0.0086 & 0.0044 & 1.0000
\end{tabular}

Table 99. Shielding tensor for $\mathrm{Si}$ in $\mathrm{Me}_{3} \mathrm{SiMe}$ in principal axes [IGLO-BP86/BIII//B3LYP/6-31+G(d)].

\begin{tabular}{c|ccc} 
& $\sigma_{1}$ & $\sigma_{2}$ & $\sigma_{3}$ \\
\hline$\sigma_{\mathrm{d}}$ & 827.0 & 827.0 & 827.0 \\
$\sigma_{\mathrm{p} 0}$ & 403.3 & 404.7 & 405.7 \\
$\sigma_{\mathrm{p} 1}$ & -898.6 & -899.7 & -900.6 \\
$\sigma$ & 331.8 & 332.1 & 332.1 \\
\hline prin. axes & & & \\
\hline $\mathrm{x}$ & -0.4075 & -0.2101 & 0.8887 \\
$\mathrm{y}$ & -0.9114 & 0.0329 & -0.4101 \\
$\mathrm{z}$ & 0.0569 & -0.9771 & -0.2049
\end{tabular}

Table 100. Shielding tensor for $\mathrm{Si}$ in (MeO)Me $\mathrm{Me}_{2} \mathrm{SiH}$ in principal axes [IGLO-BP86/BIII//B3LYP/6$31+G(d)]$.

\begin{tabular}{c|ccc} 
& $\sigma_{1}$ & $\sigma_{2}$ & $\sigma_{3}$ \\
\hline$\sigma_{\mathrm{d}}$ & 822.0 & 827.1 & 828.9 \\
$\sigma_{\mathrm{p} 0}$ & 389.5 & 400.8 & 418.4 \\
$\sigma_{\mathrm{p} 1}$ & -943.1 & -905.4 & -867.6 \\
$\sigma$ & 268.4 & 322.5 & 379.6 \\
\hline prin. axes & & & \\
\hline $\mathrm{x}$ & 0.2442 & -0.1904 & 0.9509 \\
$\mathrm{y}$ & 0.2487 & 0.9600 & 0.1284 \\
$\mathrm{z}$ & 0.9373 & -0.2051 & -0.2818
\end{tabular}

Table 101. Shielding tensor for $\mathrm{Si}$ in (MeO)Me $2 \mathrm{SiCl}$ in principal axes [IGLO-BP86/BIII//B3LYP/6$31+G(d)]$. 


\begin{tabular}{c|ccc} 
& $\sigma_{1}$ & $\sigma_{2}$ & $\sigma_{3}$ \\
\hline$\sigma_{\mathrm{d}}$ & 825.3 & 826.8 & 826.1 \\
$\sigma_{\mathrm{p} 0}$ & 379.9 & 378.0 & 396.5 \\
$\sigma_{\mathrm{p} 1}$ & -925.2 & -887.1 & -889.6 \\
$\sigma$ & 280.0 & 317.8 & 332.9 \\
\hline prin. axes & & & \\
\hline $\mathrm{x}$ & 0.1995 & -0.9555 & 0.2172 \\
$\mathrm{y}$ & -0.7999 & -0.0308 & 0.5994 \\
$\mathrm{z}$ & 0.5660 & 0.2933 & 0.7704
\end{tabular}

Table 102. Shielding tensor for $\mathrm{Si}$ in (MeO)Me ${ }_{2} \mathrm{SiLi}$ in principal axes [IGLO-BP86/BIII//B3LYP/6$31+G(d)]$.

\begin{tabular}{c|ccc} 
& $\sigma_{1}$ & $\sigma_{2}$ & $\sigma_{3}$ \\
\hline$\sigma_{\mathrm{d}}$ & 827.2 & 826.8 & 828.8 \\
$\sigma_{\mathrm{p} 0}$ & 389.0 & 401.4 & 434.6 \\
$\sigma_{\mathrm{p} 1}$ & -1034.9 & -933.5 & -911.1 \\
$\sigma$ & 181.2 & 294.7 & 352.4 \\
\hline prin. axes & & & \\
\hline $\mathrm{x}$ & 0.2930 & -0.9556 & -0.0322 \\
$\mathrm{y}$ & -0.4540 & -0.1687 & 0.8749 \\
$\mathrm{z}$ & 0.8414 & 0.2418 & 0.4832
\end{tabular}

Table 103. Shielding tensor for $\mathrm{Si}$ in (MeO)Me ${ }_{2} \mathrm{SiMe}$ in principal axes [IGLO-BP86/BIII//B3LYP/6$31+G(d)]$.

\begin{tabular}{c|ccc} 
& $\sigma_{1}$ & $\sigma_{2}$ & $\sigma_{3}$ \\
\hline$\sigma_{\mathrm{d}}$ & 825.2 & 825.9 & 827.4 \\
$\sigma_{\mathrm{p} 0}$ & 386.3 & 395.3 & 406.9 \\
$\sigma_{\mathrm{p} 1}$ & -923.9 & -920.5 & -885.8 \\
$\sigma$ & 287.6 & 300.7 & 348.5 \\
\hline prin. axes & & & \\
\hline $\mathrm{x}$ & -0.0518 & -0.9050 & 0.4222 \\
$\mathrm{y}$ & 0.3180 & -0.4157 & -0.8521 \\
$\mathrm{z}$ & -0.9467 & -0.0901 & -0.3094
\end{tabular}

Table 104. Shielding tensor for $\mathrm{Si}$ in $\left(\mathrm{Me}_{2} \mathrm{~N}\right) \mathrm{Me}_{2} \mathrm{SiH}$ in principal axes [IGLO-BP86/BIII//B3LYP/6$31+G(d)]$.

\begin{tabular}{c|ccc} 
& $\sigma_{1}$ & $\sigma_{2}$ & $\sigma_{3}$ \\
\hline$\sigma_{\mathrm{d}}$ & 821.1 & 826.8 & 829.9 \\
$\sigma_{\mathrm{p} 0}$ & 393.8 & 398.5 & 426.6 \\
$\sigma_{\mathrm{p} 1}$ & -928.9 & -877.4 & -865.6 \\
$\sigma$ & 286.0 & 347.8 & 390.9 \\
\hline prin. axes & & & \\
\hline $\mathrm{x}$ & -0.8776 & -0.4795 & 0.0004 \\
$\mathrm{y}$ & 0.0003 & 0.0003 & 1.0000 \\
$\mathrm{z}$ & 0.4795 & -0.8776 & 0.0001
\end{tabular}

Table 105. Shielding tensor for $\mathrm{Si}$ in $\left(\mathrm{Me}_{2} \mathrm{~N}\right) \mathrm{Me}_{2} \mathrm{SiCl}$ in principal axes [IGLO-BP86/BIII//B3LYP/6$31+G(d)]$.

\begin{tabular}{c|ccc} 
& $\sigma_{1}$ & $\sigma_{2}$ & $\sigma_{3}$ \\
\hline$\sigma_{\mathrm{d}}$ & 825.0 & 825.0 & 827.5 \\
$\sigma_{\mathrm{p} 0}$ & 384.4 & 379.0 & 385.2 \\
$\sigma_{\mathrm{p} 1}$ & -921.2 & -883.5 & -877.2
\end{tabular}




\begin{tabular}{c|ccc}
$\sigma$ & 288.1 & 320.5 & 335.5 \\
\hline prin. axes & & & \\
\hline$x$ & -0.5917 & -0.0302 & 0.8056 \\
$y$ & -0.0140 & -0.9988 & -0.0478 \\
$z$ & -0.8060 & 0.0396 & -0.5905
\end{tabular}

Table 106. Shielding tensor for $\mathrm{Si}$ in $\left(\mathrm{Me}_{2} \mathrm{~N}\right) \mathrm{Me}_{2} \mathrm{SiLi}$ in principal axes [IGLO-BP86/BIII/B3LYP/6$31+G(d)]$.

\begin{tabular}{c|ccc} 
& $\sigma_{1}$ & $\sigma_{2}$ & $\sigma_{3}$ \\
\hline$\sigma_{\mathrm{d}}$ & 827.5 & 825.6 & 830.7 \\
$\sigma_{\mathrm{p} 0}$ & 382.1 & 401.2 & 439.5 \\
$\sigma_{\mathrm{p} 1}$ & -986.3 & -910.8 & -914.5 \\
$\sigma$ & 223.2 & 316.0 & 355.7 \\
\hline prin. axes & & & \\
\hline $\mathrm{x}$ & 0.0000 & -1.0000 & 0.0000 \\
$\mathrm{y}$ & -0.1678 & 0.0000 & 0.9858 \\
$\mathrm{z}$ & -0.9858 & 0.0000 & -0.1678
\end{tabular}

Table 107. Shielding tensor for $\mathrm{Si}$ in $\left(\mathrm{Me}_{2} \mathrm{~N}\right) \mathrm{Me}_{2} \mathrm{SiMe}$ in principal axes [IGLO-BP86/BIII//B3LYP/6$31+G(d)]$.

\begin{tabular}{c|ccc} 
& $\sigma_{1}$ & $\sigma_{2}$ & $\sigma_{3}$ \\
\hline$\sigma_{\mathrm{d}}$ & 824.6 & 825.1 & 828.1 \\
$\sigma_{\mathrm{p} 0}$ & 399.1 & 383.3 & 403.9 \\
$\sigma_{\mathrm{p} 1}$ & -920.1 & -891.5 & -873.4 \\
$\sigma$ & 303.6 & 316.9 & 358.7 \\
\hline prin. axes & & & \\
\hline $\mathrm{x}$ & -0.2223 & 0.0901 & -0.9708 \\
$\mathrm{y}$ & -0.0164 & 0.9952 & 0.0961 \\
$\mathrm{z}$ & 0.9749 & 0.0373 & -0.2197
\end{tabular}

Table 108. Shielding tensor for $\mathrm{Si}$ in (MeS) $\mathrm{Me}_{2} \mathrm{SiH}$ in principal axes [IGLO-BP86/BIII//B3LYP/6$31+G(d)]$.

\begin{tabular}{c|ccc} 
& $\sigma_{1}$ & $\sigma_{2}$ & $\sigma_{3}$ \\
\hline$\sigma_{\mathrm{d}}$ & 823.9 & 827.5 & 830.3 \\
$\sigma_{\mathrm{p} 0}$ & 382.6 & 407.5 & 409.8 \\
$\sigma_{\mathrm{p} 1}$ & -923.8 & -905.2 & -873.7 \\
$\sigma$ & 282.7 & 329.8 & 366.4 \\
\hline prin. axes & & & \\
\hline $\mathrm{x}$ & 0.2520 & 0.4093 & 0.8769 \\
$\mathrm{y}$ & -0.0243 & -0.9032 & 0.4286 \\
$\mathrm{z}$ & 0.9674 & -0.1294 & -0.2177
\end{tabular}

Table 109. Shielding tensor for $\mathrm{Si}$ in (MeS) $\mathrm{Me}_{2} \mathrm{SiCl}$ in principal axes [IGLO-BP86/BIII//B3LYP/6$31+G(d)]$.

\begin{tabular}{c|ccc} 
& $\sigma_{1}$ & $\sigma_{2}$ & $\sigma_{3}$ \\
\hline$\sigma_{\mathrm{d}}$ & 827.2 & 828.8 & 825.9 \\
$\sigma_{\mathrm{p} 0}$ & 367.0 & 371.7 & 382.7 \\
$\sigma_{\mathrm{p} 1}$ & -939.3 & -907.9 & -902.9 \\
$\sigma$ & 254.9 & 292.5 & 305.7 \\
\hline prin. axes & & & \\
\hline $\mathrm{x}$ & -0.0548 & -0.6639 & -0.7458
\end{tabular}


Table 110. Shielding tensor for Si in (MeS)Me ${ }_{2} \mathrm{SiLi}$ in principal axes [IGLO-BP86/BIII//B3LYP/6$31+G(d)]$.

\begin{tabular}{c|ccc} 
& $\sigma_{1}$ & $\sigma_{2}$ & $\sigma_{3}$ \\
\hline$\sigma_{\mathrm{d}}$ & 829.4 & 828.9 & 830.0 \\
$\sigma_{\mathrm{p} 0}$ & 406.6 & 419.0 & 437.6 \\
$\sigma_{\mathrm{p} 1}$ & -1002.5 & -927.1 & -915.4 \\
$\sigma$ & 233.5 & 320.7 & 352.3 \\
\hline prin. axes & & & \\
\hline $\mathrm{x}$ & 0.2794 & -0.9601 & 0.0081 \\
$\mathrm{y}$ & -0.4560 & -0.1253 & 0.8811 \\
$\mathrm{z}$ & -0.8450 & -0.2499 & -0.4728
\end{tabular}

Table 111. Shielding tensor for $\mathrm{Si}$ in (MeS)Me ${ }_{2} \mathrm{SiMe}$ in principal axes [IGLO-BP86/BIII/B3LYP/6$31+G(d)]$.

\begin{tabular}{c|ccc} 
& $\sigma_{1}$ & $\sigma_{2}$ & $\sigma_{3}$ \\
\hline$\sigma_{\mathrm{d}}$ & 827.2 & 826.2 & 828.7 \\
$\sigma_{\mathrm{p} 0}$ & 378.1 & 407.0 & 390.7 \\
$\sigma_{\mathrm{p} 1}$ & -923.5 & -912.8 & -887.3 \\
$\sigma$ & 281.8 & 320.4 & 332.1 \\
\hline prin. axes & & & \\
\hline $\mathrm{x}$ & 0.1970 & -0.9804 & -0.0001 \\
$\mathrm{y}$ & -0.0025 & -0.0006 & 1.0000 \\
$\mathrm{z}$ & -0.9804 & -0.1970 & -0.0026
\end{tabular}

Table 112. Shielding tensor for $\mathrm{Si}$ in $(\mathrm{MeO})_{2} \mathrm{MeSiH}$ in principal axes [IGLO-BP86/BIII//B3LYP/6$31+G(d)]$.

\begin{tabular}{c|ccc} 
& $\sigma_{1}$ & $\sigma_{2}$ & $\sigma_{3}$ \\
\hline$\sigma_{\mathrm{d}}$ & 822.4 & 827.4 & 826.5 \\
$\sigma_{\mathrm{p} 0}$ & 395.3 & 417.0 & 404.0 \\
$\sigma_{\mathrm{p} 1}$ & -899.4 & -896.3 & -859.6 \\
$\sigma$ & 318.3 & 348.0 & 370.9 \\
\hline prin. axes & & & \\
\hline $\mathrm{x}$ & 0.2496 & 0.5579 & 0.7915 \\
$\mathrm{y}$ & 0.7969 & 0.3460 & -0.4952 \\
$\mathrm{z}$ & -0.5501 & 0.7543 & -0.3582
\end{tabular}

Table 113. Shielding tensor for $\mathrm{Si}$ in (MeO) ${ }_{2} \mathrm{MeSiCl}$ in principal axes [IGLO-BP86/BIII//B3LYP/6$31+G(d)]$.

\begin{tabular}{c|ccc} 
& $\sigma_{1}$ & $\sigma_{2}$ & $\sigma_{3}$ \\
\hline$\sigma_{\mathrm{d}}$ & 825.8 & 825.8 & 824.9 \\
$\sigma_{\mathrm{p} 0}$ & 386.2 & 384.9 & 394.0 \\
$\sigma_{\mathrm{p} 1}$ & -876.6 & -867.8 & -843.9 \\
$\sigma$ & 335.4 & 342.9 & 375.0 \\
\hline prin. axes & & & \\
\hline $\mathrm{x}$ & 0.8727 & 0.4405 & 0.2109 \\
$\mathrm{y}$ & 0.4844 & -0.7261 & -0.4881 \\
$\mathrm{z}$ & 0.0619 & -0.5281 & 0.8470
\end{tabular}


Table 114. Shielding tensor for $\mathrm{Si}$ in (MeO) ${ }_{2} \mathrm{MeSiLi}$ in principal axes [IGLO-BP86/BIII//B3LYP/6$31+G(d)]$.

\begin{tabular}{c|ccc} 
& $\sigma_{1}$ & $\sigma_{2}$ & $\sigma_{3}$ \\
\hline$\sigma_{\mathrm{d}}$ & 826.9 & 828.2 & 826.4 \\
$\sigma_{\mathrm{p} 0}$ & 388.5 & 413.2 & 413.0 \\
$\sigma_{\mathrm{p} 1}$ & -992.0 & -968.0 & -875.1 \\
$\sigma$ & 223.4 & 273.4 & 364.2 \\
\hline prin. axes & & & \\
\hline $\mathrm{x}$ & 0.0727 & -0.9851 & 0.1562 \\
$\mathrm{y}$ & -0.9143 & -0.0032 & 0.4051 \\
$\mathrm{z}$ & 0.3986 & 0.1722 & 0.9008
\end{tabular}

Table 115. Shielding tensor for $\mathrm{Si}$ in $(\mathrm{MeO})_{2} \mathrm{MeSiMe}$ in principal axes [IGLO-BP86/BIII//B3LYP/6$31+G(d)]$.

\begin{tabular}{c|ccc} 
& $\sigma_{1}$ & $\sigma_{2}$ & $\sigma_{3}$ \\
\hline$\sigma_{\mathrm{d}}$ & 825.6 & 826.0 & 825.2 \\
$\sigma_{\mathrm{p} 0}$ & 390.4 & 397.6 & 400.9 \\
$\sigma_{\mathrm{p} 1}$ & -896.2 & -903.3 & -867.1 \\
$\sigma$ & 319.8 & 320.4 & 359.0 \\
\hline prin. axes & & & \\
\hline $\mathrm{x}$ & 0.0169 & 0.9998 & -0.0082 \\
$\mathrm{y}$ & 0.8937 & -0.0188 & -0.4484 \\
$\mathrm{z}$ & 0.4484 & -0.0002 & 0.8938
\end{tabular}

Table 116. Shielding tensor for $\mathrm{Si}$ in $\left(\mathrm{Me}_{2} \mathrm{~N}\right)_{2} \mathrm{MeSiH}$ in principal axes [IGLO-BP86/BIII//B3LYP/6$31+G(d)]$.

\begin{tabular}{c|ccc} 
& $\sigma_{1}$ & $\sigma_{2}$ & $\sigma_{3}$ \\
\hline$\sigma_{\mathrm{d}}$ & 820.3 & 828.6 & 824.0 \\
$\sigma_{\mathrm{p} 0}$ & 372.2 & 424.6 & 392.7 \\
$\sigma_{\mathrm{p} 1}$ & -867.2 & -915.6 & -838.7 \\
$\sigma$ & 325.2 & 337.6 & 378.1 \\
\hline prin. axes & & & \\
\hline $\mathrm{x}$ & -0.3101 & -0.5371 & -0.7845 \\
$\mathrm{y}$ & 0.9504 & -0.1967 & -0.2410 \\
$\mathrm{z}$ & -0.0249 & -0.8203 & 0.5714
\end{tabular}

Table 117. Shielding tensor for $\mathrm{Si}$ in $\left(\mathrm{Me}_{2} \mathrm{~N}\right)_{2} \mathrm{MeSiCl}$ in principal axes [IGLO-BP86/BIII//B3LYP/6$31+G(d)]$.

\begin{tabular}{c|ccc} 
& $\sigma_{1}$ & $\sigma_{2}$ & $\sigma_{3}$ \\
\hline$\sigma_{\mathrm{d}}$ & 822.7 & 824.2 & 825.3 \\
$\sigma_{\mathrm{p} 0}$ & 362.9 & 379.0 & 375.5 \\
$\sigma_{\mathrm{p} 1}$ & -869.9 & -866.8 & -838.1 \\
$\sigma$ & 315.7 & 336.4 & 362.7 \\
\hline prin. axes & & & \\
\hline $\mathrm{x}$ & -0.2493 & 0.8507 & -0.4629 \\
$\mathrm{y}$ & 0.3916 & 0.5257 & 0.7552 \\
$\mathrm{z}$ & -0.8857 & -0.0070 & 0.4641
\end{tabular}

Table 118. Shielding tensor for $\mathrm{Si}$ in $\left(\mathrm{Me}_{2} \mathrm{~N}\right)_{2} \mathrm{MeSiLi}$ in principal axes [IGLO-BP86/BIII/B3LYP/6$31+G(d)]$.

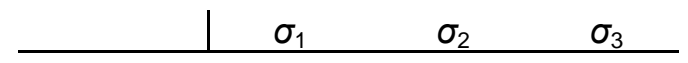




\begin{tabular}{c|ccc}
\hline$\sigma_{\mathrm{d}}$ & 826.2 & 829.1 & 824.0 \\
$\sigma_{\mathrm{p} 0}$ & 384.9 & 417.0 & 389.0 \\
$\sigma_{\mathrm{p} 1}$ & -990.2 & -936.2 & -870.9 \\
$\sigma$ & 221.0 & 309.9 & 342.1 \\
\hline prin. axes & & & \\
\hline $\mathrm{x}$ & -0.0113 & -0.9997 & 0.0203 \\
$\mathrm{y}$ & -0.9610 & 0.0165 & 0.2760 \\
$\mathrm{z}$ & 0.2763 & 0.0164 & 0.9609
\end{tabular}

Table 119. Shielding tensor for $\mathrm{Si}$ in $\left(\mathrm{Me}_{2} \mathrm{~N}\right)_{2} \mathrm{MeSiMe}$ in principal axes [IGLO-BP86/BIII//B3LYP/6$31+G(d)]$.

\begin{tabular}{c|ccc} 
& $\sigma_{1}$ & $\sigma_{2}$ & $\sigma_{3}$ \\
\hline$\sigma_{\mathrm{d}}$ & 823.1 & 824.4 & 825.0 \\
$\sigma_{\mathrm{p} 0}$ & 385.4 & 388.8 & 393.6 \\
$\sigma_{\mathrm{p} 1}$ & -882.7 & -884.3 & -861.2 \\
$\sigma$ & 325.8 & 328.8 & 357.4 \\
\hline prin. axes & & & \\
\hline $\mathrm{x}$ & 0.5209 & 0.0365 & -0.8528 \\
$\mathrm{y}$ & 0.0193 & -0.9993 & -0.0310 \\
$\mathrm{z}$ & -0.8534 & -0.0004 & -0.5213
\end{tabular}

Table 120. Shielding tensor for $\mathrm{Si}$ in (MeS) ${ }_{2} \mathrm{MeSiH}$ in principal axes [IGLO-BP86/BIII/B3LYP/6$31+G(d)]$.

\begin{tabular}{c|ccc} 
& $\sigma_{1}$ & $\sigma_{2}$ & $\sigma_{3}$ \\
\hline$\sigma_{\mathrm{d}}$ & 824.4 & 829.2 & 829.4 \\
$\sigma_{\mathrm{p} 0}$ & 350.6 & 404.9 & 407.6 \\
$\sigma_{\mathrm{p} 1}$ & -942.9 & -892.5 & -867.3 \\
$\sigma$ & 232.2 & 341.6 & 369.6 \\
\hline prin. axes & & & \\
\hline $\mathrm{x}$ & 0.1094 & 0.2676 & 0.9573 \\
$\mathrm{y}$ & 0.7122 & -0.6929 & 0.1123 \\
$\mathrm{z}$ & -0.6933 & -0.6695 & 0.2664
\end{tabular}

Table 121. Shielding tensor for $\mathrm{Si}$ in (MeS) ${ }_{2} \mathrm{MeSiCl}$ in principal axes [IGLO-BP86/BIII//B3LYP/6$31+G(d)]$.

\begin{tabular}{c|ccc} 
& $\sigma_{1}$ & $\sigma_{2}$ & $\sigma_{3}$ \\
\hline$\sigma_{\mathrm{d}}$ & 827.5 & 827.8 & 827.7 \\
$\sigma_{\mathrm{p} 0}$ & 343.8 & 358.8 & 363.3 \\
$\sigma_{\mathrm{p} 1}$ & -942.6 & -886.0 & -881.6 \\
$\sigma$ & 228.7 & 300.7 & 309.4 \\
\hline prin. axes & & & \\
\hline $\mathrm{x}$ & 0.0821 & 0.3211 & 0.9435 \\
$\mathrm{y}$ & 0.8326 & 0.4982 & -0.2420 \\
$\mathrm{z}$ & -0.5478 & 0.8054 & -0.2264
\end{tabular}

Table 122. Shielding tensor for $\mathrm{Si}$ in (MeS) $)_{2}$ MeSiLi in principal axes [IGLO-BP86/BIII//B3LYP/6$31+G(d)]$.

\begin{tabular}{c|ccc} 
& $\sigma_{1}$ & $\sigma_{2}$ & $\sigma_{3}$ \\
\hline$\sigma_{\mathrm{d}}$ & 830.6 & 829.9 & 830.5 \\
$\sigma_{\mathrm{p} 0}$ & 403.7 & 408.4 & 426.5 \\
$\sigma_{\mathrm{p} 1}$ & -1005.2 & -932.2 & -928.6 \\
$\sigma$ & 229.1 & 306.1 & 328.3 \\
\hline
\end{tabular}




\begin{tabular}{c|ccc}
\hline prin. axes & & & \\
\hline$x$ & -0.0020 & -0.5718 & 0.8204 \\
$y$ & 0.7980 & 0.4935 & 0.3459 \\
$z$ & 0.6026 & -0.6554 & -0.4553
\end{tabular}

Table 123. Shielding tensor for $\mathrm{Si}$ in (MeS) ${ }_{2} \mathrm{MeSiMe}$ in principal axes [IGLO-BP86/BIII//B3LYP/6$31+G(d)]$.

\begin{tabular}{c|ccc} 
& $\sigma_{1}$ & $\sigma_{2}$ & $\sigma_{3}$ \\
\hline$\sigma_{\mathrm{d}}$ & 827.3 & 828.2 & 827.7 \\
$\sigma_{\mathrm{p} 0}$ & 356.0 & 392.0 & 379.9 \\
$\sigma_{\mathrm{p} 1}$ & -943.1 & -901.4 & -884.1 \\
$\sigma$ & 240.2 & 318.9 & 323.6 \\
\hline prin. axes & & & \\
\hline $\mathrm{x}$ & 0.0514 & -0.0001 & 0.9987 \\
$\mathrm{y}$ & 0.9987 & 0.0036 & -0.0514 \\
$\mathrm{z}$ & -0.0036 & 1.0000 & 0.0003
\end{tabular}

Table 124. Shielding tensor for $\mathrm{Si}$ in $(\mathrm{MeO})_{3} \mathrm{MeSiCl}$ in principal axes [IGLO-BP86/BIII//B3LYP/6$31+G(d)]$.

\begin{tabular}{c|ccc} 
& $\sigma_{1}$ & $\sigma_{2}$ & $\sigma_{3}$ \\
\hline$\sigma_{\mathrm{d}}$ & 823.9 & 825.4 & 825.4 \\
$\sigma_{\mathrm{p} 0}$ & 400.3 & 399.7 & 393.1 \\
$\sigma_{\mathrm{p} 1}$ & -833.6 & -828.8 & -803.4 \\
$\sigma$ & 390.6 & 396.3 & 415.0 \\
\hline prin. axes & & & \\
\hline $\mathrm{x}$ & -0.4625 & -0.2130 & -0.8606 \\
$\mathrm{y}$ & 0.4849 & 0.7519 & -0.4467 \\
$\mathrm{z}$ & 0.7423 & -0.6239 & -0.2445
\end{tabular}

Table 125. Shielding tensor for $\mathrm{Si}$ in $(\mathrm{MeO})_{3} \mathrm{MeSiLi}$ in principal axes [IGLO-BP86/BIII//B3LYP/6$31+G(d)]$.

\begin{tabular}{c|ccc} 
& $\sigma_{1}$ & $\sigma_{2}$ & $\sigma_{3}$ \\
\hline$\sigma_{\mathrm{d}}$ & 827.5 & 826.8 & 824.9 \\
$\sigma_{\mathrm{p} 0}$ & 397.0 & 395.2 & 415.2 \\
$\sigma_{\mathrm{p} 1}$ & -930.5 & -908.3 & -823.7 \\
$\sigma$ & 293.9 & 313.7 & 416.4 \\
\hline prin. axes & & & \\
\hline $\mathrm{x}$ & -0.6746 & 0.6867 & 0.2709 \\
$\mathrm{y}$ & 0.7368 & 0.6489 & 0.1900 \\
$\mathrm{z}$ & 0.0453 & -0.3278 & 0.9437
\end{tabular}

Table 126. Shielding tensor for $\mathrm{Si}$ in $\left(\mathrm{Me}_{2} \mathrm{~N}\right)_{3} \mathrm{MeSiCl}$ in principal axes [IGLO-BP86/BIII//B3LYP/6$31+G(d)]$.

\begin{tabular}{c|ccc} 
& $\sigma_{1}$ & $\sigma_{2}$ & $\sigma_{3}$ \\
\hline$\sigma_{\mathrm{d}}$ & 818.2 & 823.3 & 823.0 \\
$\sigma_{\mathrm{p} 0}$ & 353.6 & 377.6 & 373.7 \\
$\sigma_{\mathrm{p} 1}$ & -838.6 & -829.3 & -814.9 \\
$\sigma$ & 333.2 & 371.6 & 381.8 \\
\hline prin. axes & & & \\
\hline $\mathrm{x}$ & 0.0916 & -0.0559 & 0.9942 \\
$\mathrm{y}$ & 0.5073 & -0.8565 & -0.0949 \\
$\mathrm{z}$ & 0.8569 & 0.5131 & -0.0501
\end{tabular}


Table 127. Shielding tensor for $\mathrm{Si}$ in $\left(\mathrm{Me}_{2} \mathrm{~N}\right)_{3} \mathrm{MeSiLi}$ in principal axes [IGLO-BP86/BIII/B3LYP/6$31+G(d)]$.

\begin{tabular}{c|ccc} 
& $\sigma_{1}$ & $\sigma_{2}$ & $\sigma_{3}$ \\
\hline$\sigma_{\mathrm{d}}$ & 828.2 & 825.9 & 821.9 \\
$\sigma_{\mathrm{p} 0}$ & 404.9 & 395.9 & 384.5 \\
$\sigma_{\mathrm{p} 1}$ & -960.6 & -928.4 & -840.9 \\
$\sigma$ & 272.5 & 293.3 & 365.5 \\
\hline prin. axes & & & \\
\hline $\mathrm{x}$ & 0.9998 & -0.0204 & -0.0087 \\
$\mathrm{y}$ & -0.0197 & -0.9971 & 0.0734 \\
$\mathrm{z}$ & 0.0101 & 0.0732 & 0.9973
\end{tabular}

Table 128. Shielding tensor for $\mathrm{Si}$ in (MeS) ${ }_{3} \mathrm{MeSiCl}$ in principal axes [IGLO-BP86/BIII//B3LYP/6$31+G(d)]$.

\begin{tabular}{c|ccc} 
& $\sigma_{1}$ & $\sigma_{2}$ & $\sigma_{3}$ \\
\hline$\sigma_{\mathrm{d}}$ & 828.0 & 828.5 & 827.6 \\
$\sigma_{\mathrm{p} 0}$ & 326.8 & 342.7 & 344.8 \\
$\sigma_{\mathrm{p} 1}$ & -915.4 & -891.6 & -849.1 \\
$\sigma$ & 239.4 & 279.6 & 323.3 \\
\hline prin. axes & & & \\
\hline $\mathrm{x}$ & 0.3806 & 0.2890 & 0.8784 \\
$\mathrm{y}$ & 0.3398 & -0.9272 & 0.1578 \\
$\mathrm{z}$ & -0.8601 & -0.2384 & 0.4511
\end{tabular}

Table 129. Shielding tensor for $\mathrm{Si}$ in (MeS) $)_{3} \mathrm{MeSiLi}$ in principal axes [IGLO-BP86/BIII//B3LYP/6$31+G(d)]$.

\begin{tabular}{c|ccc} 
& $\sigma_{1}$ & $\sigma_{2}$ & $\sigma_{3}$ \\
\hline$\sigma_{\mathrm{d}}$ & 832.0 & 830.8 & 830.0 \\
$\sigma_{\mathrm{p} 0}$ & 398.5 & 378.6 & 407.7 \\
$\sigma_{\mathrm{p} 1}$ & -1007.3 & -949.9 & -919.9 \\
$\sigma$ & 223.1 & 259.5 & 317.9 \\
\hline prin. axes & & & \\
\hline $\mathrm{x}$ & -0.7737 & 0.1707 & -0.6101 \\
$\mathrm{y}$ & 0.1100 & -0.9122 & -0.3947 \\
$\mathrm{z}$ & -0.6239 & -0.3726 & 0.6870
\end{tabular}

Table 130. MO contributions to $\sigma$ in principal axes for Si in 4.3 THF [IGLO-BP86/mixed basis//B3LYP/TZVP].

\begin{tabular}{cccccc} 
MO & type & $\sigma_{1}$ & $\sigma_{2}$ & $\sigma_{3}$ & average \\
\hline 1 & AO Si(7) & 481.9 & 481.9 & 481.9 & 481.9 \\
2 & AO O(20) & 0.0 & 0.0 & 0.0 & 0.0 \\
3 & AO O(30) & 0.0 & 0.0 & 0.0 & 0.0 \\
4 & AO O(25) & 0.0 & 0.0 & 0.0 & 0.0 \\
5 & AO N(14) & 0.0 & 0.0 & 0.0 & 0.0 \\
6 & AO C(24) & 0.0 & 0.0 & 0.0 & 0.0 \\
7 & AO C(21) & 0.0 & 0.0 & 0.0 & 0.0 \\
8 & AO C(31) & 0.0 & 0.0 & 0.0 & 0.0 \\
9 & AO C(34) & 0.0 & 0.0 & 0.0 & 0.0 \\
10 & AO C(26) & 0.0 & 0.0 & 0.0 & 0.0 \\
11 & AO C(29) & 0.0 & 0.0 & 0.0 & 0.0 \\
12 & AO C (23) & 0.0 & 0.0 & 0.0 & 0.0
\end{tabular}




\begin{tabular}{|c|c|c|c|c|c|}
\hline 13 & $\mathrm{AO} C(22)$ & 0.0 & 0.0 & 0.0 & 0.0 \\
\hline 14 & AO C(33) & 0.0 & 0.0 & 0.0 & 0.0 \\
\hline 15 & $\mathrm{AO} C(32)$ & 0.0 & 0.0 & 0.0 & 0.0 \\
\hline 16 & AO C(28) & 0.0 & 0.0 & 0.0 & 0.0 \\
\hline 17 & AO C (27) & 0.0 & 0.0 & 0.0 & 0.0 \\
\hline 18 & AO C(17) & 0.0 & 0.0 & 0.0 & 0.0 \\
\hline 19 & AO C(11) & 0.0 & 0.0 & 0.0 & 0.0 \\
\hline 20 & $A O C(5)$ & 0.0 & 0.0 & 0.0 & 0.0 \\
\hline 21 & AO C (10) & 0.0 & 0.0 & 0.0 & 0.0 \\
\hline 22 & $\mathrm{AO} C(6)$ & 0.0 & 0.0 & 0.0 & 0.0 \\
\hline 23 & AO C (1) & 0.0 & 0.0 & 0.0 & 0.0 \\
\hline 24 & AO C(12) & 0.0 & 0.0 & 0.0 & 0.0 \\
\hline 25 & AO C(15) & 0.0 & 0.0 & 0.0 & 0.0 \\
\hline 26 & $\mathrm{AO} C(9)$ & 0.0 & 0.0 & 0.0 & 0.0 \\
\hline 27 & $\mathrm{AO} C(2)$ & 0.0 & 0.0 & 0.0 & 0.0 \\
\hline 28 & AO C(4) & 0.0 & 0.0 & 0.0 & 0.0 \\
\hline 29 & AO C(13) & 0.0 & 0.0 & 0.0 & 0.0 \\
\hline 30 & AO C(18) & 0.0 & 0.0 & 0.0 & 0.0 \\
\hline 31 & AO C(16) & 0.0 & 0.0 & 0.0 & 0.0 \\
\hline 32 & $\mathrm{AO} C(3)$ & 0.0 & -0.1 & 0.0 & 0.0 \\
\hline 33 & AO C(8) & 0.0 & 0.0 & -0.1 & 0.0 \\
\hline 34 & AO Si(7) & 77.6 & 78.7 & 71.1 & 75.8 \\
\hline 35 & AO Si(7) & 76.5 & 81.9 & 69.7 & 76 \\
\hline 36 & AO Si(7) & 68.2 & 66.9 & 91.4 & 75. \\
\hline 37 & AO Si(7) & 72.3 & 64.2 & 88.8 & 75. \\
\hline 38 & AO Li(19) & 0.0 & -0.1 & 0.1 & 0.0 \\
\hline 39 & BOND H(50)-C(24) & 0.0 & -0.1 & 0.1 & 0.0 \\
\hline 40 & BOND H(62)-C(31) & -0.3 & 0.0 & 0.1 & -0 . \\
\hline 41 & BOND H(66)-C(28) & 0.0 & 0.0 & 0.0 & 0.0 \\
\hline 42 & BOND N(14)-C(17) & -3.7 & 2.0 & -3.1 & -1 \\
\hline 43 & BOND H(58)-C(11) & 0.0 & 0.0 & 0.0 & 0. \\
\hline 44 & BOND C(12)-C(13) & -0.5 & -1.5 & -1.2 & -1 \\
\hline 45 & BOND H(49)-C(21) & -0.1 & 0.0 & 0.0 & 0.0 \\
\hline 46 & BOND H(77)-C(33) & -0.1 & 0.0 & 0.1 & 0.0 \\
\hline 47 & BOND H(59)-C(27) & 0.1 & 0.0 & 0.1 & 0.0 \\
\hline 48 & BOND H(37)-C(22) & 0.0 & 0.0 & 0.0 & 0.0 \\
\hline 49 & BOND H(64)-C(31) & 0.0 & -0.1 & 0.1 & 0.0 \\
\hline 50 & LP O(25) & 0.2 & 0.4 & 0.1 & 0.2 \\
\hline 51 & BOND C(16)-C(15) & -1.5 & -0.7 & -1.1 & -1 \\
\hline 52 & BOND H(67)-C(5) & -0.1 & 0.1 & 0.0 & 0. \\
\hline 53 & BOND H(55)-C(10) & -0.1 & 0.0 & 0.1 & 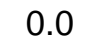 \\
\hline 54 & BOND H(78)-C(1) & 0.0 & 0.1 & 0.0 & 0. \\
\hline 55 & BOND C(5)-C(4) & -0.4 & -1.7 & -0.9 & -1 . \\
\hline 56 & BOND H(38)-C(16) & 0.0 & 0.0 & 0.1 & 0.1 \\
\hline 57 & BOND H(47)-C(23) & 0.0 & 0.0 & 0.0 & $0 . c$ \\
\hline 58 & BOND C(22)-C(21) & -0.1 & -0.1 & 0.0 & 0.0 \\
\hline 59 & BOND H(69)-C(32) & 0.0 & 0.1 & 0.1 & 0.0 \\
\hline 60 & BOND O(25)-C(29) & 0.0 & 0.0 & 0.1 & 0. \\
\hline 61 & BOND H(54)-C(26) & -0.1 & 0.0 & 0.0 & 0.0 \\
\hline 62 & BOND H(35)-C(18) & 0.2 & 0.1 & 0.2 & 0.2 \\
\hline 63 & BOND C(28)-C(27) & 0.1 & 0.0 & 0.0 & 0.0 \\
\hline 64 & BOND H(76)-C(6) & 0.1 & 0.1 & 0.1 & 0. \\
\hline 65 & BOND C(10)-C(11) & -0.1 & -0.1 & 0.0 & -0 \\
\hline 66 & BOND H(61)-C(12) & 0.0 & -0.1 & -0.1 & -0 \\
\hline 67 & BOND C (6)-C(1) & -0.1 & -0.1 & -0.1 & -0 \\
\hline 68 & BOND H(45)-C(17) & -2.6 & 0.6 & -0.5 & -0 \\
\hline 69 & BOND H(53)-C(24) & 0.0 & 0.0 & 0.1 & 0. \\
\hline 70 & BOND C(23)-C(24) & -0.1 & -0.1 & 0.1 & 0. \\
\hline 71 & BOND H(75)-C(33) & 0.0 & 0.0 & 0.0 & $0 .(-1+x-2)-x$ \\
\hline 72 & BOND H(51)-C(26) & 0.0 & -0.1 & 0.0 & $0 .(-1+x-2)-x$ \\
\hline 73 & BOND H(68)-C(32) & -0.1 & 0.0 & 0.0 & 0.0 \\
\hline 74 & BOND H(71)-C(29) & 0.1 & 0.0 & 0.0 & 0.0 \\
\hline
\end{tabular}




\begin{tabular}{|c|c|c|c|c|c|}
\hline 75 & BOND H(60)-C(4) & -0.2 & -0.3 & -1.2 & -0.5 \\
\hline 76 & BOND C(12)-C(11) & 0.2 & 0.0 & 0.7 & 0.3 \\
\hline 77 & BOND C (10)-C(9) & 0.0 & -0.6 & -0.1 & -0.3 \\
\hline 78 & BOND H(39)-C(23) & 0.0 & 0.0 & 0.0 & 0.0 \\
\hline 79 & BOND H(74)-C(34) & 0.0 & 0.1 & 0.0 & 0.0 \\
\hline 80 & LP O(20) & -0.2 & -0.1 & 0.2 & 0.0 \\
\hline 81 & BOND O(30)-C(34) & 0.1 & 0.0 & 0.1 & 0.1 \\
\hline 82 & BOND O(30)-C(31) & 0.0 & 0.0 & 0.1 & 0.1 \\
\hline 83 & BOND H(44)-C(15) & 0.1 & 0.4 & 1.0 & 0.5 \\
\hline 84 & BOND C(26)-C(27) & 0.1 & 0.0 & 0.0 & 0.0 \\
\hline 85 & BOND C $(10)-C(9)$ & 0.1 & -0.6 & 0.2 & -0.1 \\
\hline 86 & BOND H(46)-C(22) & 0.0 & 0.0 & 0.0 & 0.0 \\
\hline 87 & BOND C(8)-C(13) & 1.4 & -3.6 & -2.7 & -1.6 \\
\hline 88 & BOND C(33)-C(32) & 0.0 & 0.1 & 0.0 & 0.0 \\
\hline 89 & BOND C(4)-C(3) & -0.3 & -1.4 & -6.5 & -2.7 \\
\hline 90 & BOND H(65)-C(28) & 0.0 & -0.1 & 0.0 & 0.0 \\
\hline 91 & BOND C(8)-C(13) & -0.7 & -2.0 & -2.5 & -1.7 \\
\hline 92 & BOND H(48)-C(16) & -0.1 & 0.0 & 0.0 & 0.0 \\
\hline 93 & BOND H(40)-C(18) & 0.0 & 0.2 & -0.3 & -0.1 \\
\hline 94 & BOND C (1)-C(2) & -1.0 & -0.1 & -0.2 & -0.4 \\
\hline 95 & BOND C(2)-C(3) & -1.1 & -2.6 & -4.1 & -2.6 \\
\hline 96 & BOND H(42)-C(21) & 0.1 & 0.0 & 0.0 & 0.0 \\
\hline 97 & BOND H(43)-C(16) & 0.1 & 0.1 & -0.1 & 0.0 \\
\hline 98 & BOND H(52)-C(15) & -1.4 & -0.6 & -0.1 & -0.7 \\
\hline 99 & BOND O(20)-C(24) & 0.1 & 0.0 & 0.1 & 0.0 \\
\hline 100 & BOND C(33)-C(34) & 0.0 & 0.0 & 0.0 & 0.0 \\
\hline 101 & LP O(25) & -0.2 & -0.5 & -0.1 & -0.3 \\
\hline 102 & BOND H(70)-C(29) & 0.0 & -0.1 & -0.1 & -0.1 \\
\hline 103 & BOND C (28)-C(29) & 0.1 & 0.0 & -0.1 & 0.0 \\
\hline 104 & BOND H(63)-C(13) & -0.4 & -1.3 & -0.5 & -0.7 \\
\hline 105 & BOND C(23)-C(22) & 0.0 & 0.0 & 0.0 & 0.0 \\
\hline 106 & BOND O(20)-C(21) & 0.0 & 0.0 & 0.2 & 0.1 \\
\hline 107 & BOND C(18)-C(17) & -0.1 & 1.1 & -0.1 & 0.3 \\
\hline 108 & BOND H(73)-C(34) & 0.0 & -0.1 & 0.0 & 0.0 \\
\hline 109 & BOND H(57)-C(27) & 0.0 & -0.1 & -0.1 & -0.1 \\
\hline 110 & BOND C(31)-C(32) & -0.1 & 0.0 & 0.0 & 0.0 \\
\hline 111 & BOND C(4)-C(3) & -0.6 & 1.1 & -3.2 & -0.9 \\
\hline 112 & BOND N(14)-Si(7) & -102.6 & -35.2 & -103.1 & -80.3 \\
\hline 113 & LP O(30) & 0.3 & 0.2 & 0.1 & 0.2 \\
\hline 114 & BOND C(6)-C(5) & 0.1 & 0.1 & -0.5 & -0.1 \\
\hline 115 & BOND H(41)-C(17) & -1.7 & 0.4 & 0.1 & -0.4 \\
\hline 116 & BOND C(12)-C(11) & 0.2 & 0.5 & 0.5 & 0.4 \\
\hline 117 & BOND H(36)-C(18) & 0.1 & 0.2 & 0.0 & 0.1 \\
\hline 118 & BOND C (6)-C(5) & -0.1 & -0.1 & 0.0 & 0.0 \\
\hline 119 & BOND H(56)-C(9) & -0.2 & -2.1 & 0.1 & -0.7 \\
\hline 120 & BOND N(14)-C(15) & -6.4 & -1.2 & -5.2 & -4.3 \\
\hline 121 & LP O(20) & -0.1 & -0.1 & 0.0 & -0.1 \\
\hline 122 & LP O(30) & -0.6 & -0.4 & 0.0 & -0.3 \\
\hline 123 & BOND O(25)-C(26) & 0.1 & 0.0 & 0.1 & 0.1 \\
\hline 124 & BOND H(72)-C(2) & -0.5 & 0.1 & -0.9 & -0.4 \\
\hline 125 & BOND C(2)-C(1) & 0.5 & -0.3 & -0.4 & 0.0 \\
\hline 126 & BOND C(3)-Si(7) & -88.4 & -107.1 & -132.1 & -109.2 \\
\hline 127 & BOND C (9)-C(8) & -0.9 & -2.3 & -1.6 & -1.6 \\
\hline 128 & BOND C (8)-Si(7) & -52.4 & -96.7 & -154.9 & -101.3 \\
\hline 129 & LP N(14) & -10.1 & 3.4 & -8.8 & -5.2 \\
\hline 130 & BOND Si(7)-Li(19) & -278.9 & -206.9 & 7.4 & -159.5 \\
\hline
\end{tabular}

Table 131. MO contributions to $\sigma$ in principal axes for Si in 5.3 THF [IGLO-BP86/mixed basis//B3LYP/TZVP]. 


\begin{tabular}{|c|c|c|c|c|c|}
\hline $\mathrm{MO}$ & type & $\sigma_{1}$ & $\sigma_{2}$ & $\sigma_{3}$ & average \\
\hline 1 & AO Si(43) & 481.9 & 481.9 & 481.9 & 481.9 \\
\hline 2 & $\mathrm{AO} O(40)$ & 0.0 & 0.0 & 0.0 & 0.0 \\
\hline 3 & $\mathrm{AO} O(62)$ & 0.0 & 0.0 & 0.0 & 0.0 \\
\hline 4 & $\mathrm{AO} O(32)$ & 0.0 & 0.0 & 0.0 & 0.0 \\
\hline 5 & AO N(29) & 0.0 & 0.0 & 0.0 & 0.0 \\
\hline 6 & AO N(55) & 0.0 & 0.0 & 0.0 & 0.0 \\
\hline 7 & AO C(66) & 0.0 & 0.0 & 0.0 & 0.0 \\
\hline 8 & $\mathrm{AO} C(47)$ & 0.0 & 0.0 & 0.0 & 0.0 \\
\hline 9 & $\mathrm{AO} C(27)$ & 0.0 & 0.0 & 0.0 & 0.0 \\
\hline 10 & AO C(33) & 0.0 & 0.0 & 0.0 & 0.0 \\
\hline 11 & AO C(73) & 0.0 & 0.0 & 0.0 & 0.0 \\
\hline 12 & AO C(19) & 0.0 & 0.0 & 0.0 & 0.0 \\
\hline 13 & AO C(77) & 0.0 & 0.0 & 0.0 & 0.0 \\
\hline 14 & AO C(24) & 0.0 & 0.0 & 0.0 & 0.0 \\
\hline 15 & AO C (20) & 0.0 & 0.0 & 0.0 & 0.0 \\
\hline 16 & AO C(80) & 0.0 & 0.0 & 0.0 & 0.0 \\
\hline 17 & AO C(35) & 0.0 & 0.0 & 0.0 & 0.0 \\
\hline 18 & AO C(14) & 0.0 & 0.0 & 0.0 & 0.0 \\
\hline 19 & AO C(15) & 0.0 & 0.0 & 0.0 & 0.0 \\
\hline 20 & AO C(58) & 0.0 & 0.0 & 0.0 & 0.0 \\
\hline 21 & AO C (23) & 0.0 & 0.0 & 0.0 & 0.0 \\
\hline 22 & AO C(56) & 0.0 & 0.0 & 0.0 & 0.0 \\
\hline 23 & AO C(59) & 0.0 & 0.0 & 0.0 & 0.0 \\
\hline 24 & AO C(48) & 0.0 & 0.0 & 0.0 & 0.0 \\
\hline 25 & AO C(67) & 0.0 & 0.0 & 0.0 & 0.0 \\
\hline 26 & $\mathrm{AO} C(42)$ & 0.0 & 0.0 & 0.0 & 0.0 \\
\hline 27 & AO C(69) & 0.0 & 0.0 & 0.0 & 0.0 \\
\hline 28 & AO C(63) & 0.0 & 0.0 & 0.0 & 0.0 \\
\hline 29 & AO C(13) & 0.0 & 0.0 & 0.0 & 0.0 \\
\hline 30 & $A O C(5)$ & 0.0 & 0.0 & 0.0 & 0.0 \\
\hline 31 & $\mathrm{AO} C(45)$ & 0.0 & 0.0 & 0.0 & 0.0 \\
\hline 32 & AO C(51) & 0.0 & -0.1 & 0.0 & 0.0 \\
\hline 33 & AO Si(43) & 70.6 & 69.8 & 87.3 & 75.9 \\
\hline 34 & AO Si(43) & 88.6 & 85.9 & 62.6 & 79.0 \\
\hline 35 & AO Si(43) & 77.2 & 67.5 & 84.1 & 76.3 \\
\hline 36 & AO Si(43) & 64.8 & 69.5 & 94.1 & 76.1 \\
\hline 37 & AO Li(46) & -0.1 & -0.1 & 0.1 & 0.0 \\
\hline 38 & BOND H(11)-C(20) & 0.0 & 0.0 & 0.0 & 0.0 \\
\hline 39 & BOND H(28)-C(24) & 0.0 & 0.0 & 0.0 & 0.0 \\
\hline 40 & LP O(32) & 0.2 & 0.2 & 0.1 & 0.2 \\
\hline 41 & BOND H(72)-C(69) & -0.1 & 0.2 & -0.1 & 0.0 \\
\hline 42 & BOND $\mathrm{H}(7)-\mathrm{C}(5)$ & -0.1 & 0.1 & -0.1 & 0.0 \\
\hline 43 & BOND C(59)-C(67) & -0.1 & 0.0 & -0.1 & -0.1 \\
\hline 44 & BOND H(3)-C(14) & 0.1 & 0.0 & 0.0 & 0.0 \\
\hline 45 & BOND H(82)-C(80) & 0.0 & 0.1 & 0.0 & 0.0 \\
\hline 46 & BOND O(40)-C(47) & 0.0 & 0.0 & 0.1 & 0.1 \\
\hline 47 & BOND H(50)-C(47) & 0.0 & 0.0 & 0.0 & 0.0 \\
\hline 48 & BOND H(79)-C(80) & 0.0 & 0.0 & 0.0 & 0.0 \\
\hline 49 & BOND H(81)-C(77) & 0.0 & 0.0 & 0.0 & 0.0 \\
\hline 50 & BOND H(65)-C(56) & -0.5 & -0.9 & -0.3 & -0.6 \\
\hline 51 & BOND H(4)-C(15) & -0.2 & -0.6 & -0.1 & -0.3 \\
\hline 52 & BOND H(60)-C(59) & 0.1 & 0.1 & 0.0 & 0.1 \\
\hline 53 & BOND H(68)-C(63) & -0.3 & -0.5 & -1.4 & -0.7 \\
\hline 54 & BOND H(44)-C(45) & 0.4 & 0.0 & 0.2 & 0.2 \\
\hline 55 & BOND H(31)-C(23) & -0.4 & -0.8 & -1.1 & -0.8 \\
\hline 56 & BOND H(16)-C(27) & -0.1 & -0.1 & 0.1 & 0.0 \\
\hline 57 & BOND H(22)-C(14) & -0.1 & 0.0 & 0.0 & 0.0 \\
\hline 58 & BOND H(61)-C(66) & 0.0 & -0.1 & 0.0 & 0.0 \\
\hline 59 & BOND H(70)-C(73) & -0.2 & -0.3 & -0.1 & -0.2 \\
\hline 60 & BOND H(36)-C(35) & 0.1 & 0.0 & 0.1 & 0.1 \\
\hline 61 & BOND H(30)-C(33) & 0.1 & 0.0 & 0.0 & 0.0 \\
\hline
\end{tabular}




\begin{tabular}{|c|c|c|c|c|c|}
\hline 62 & BOND H(71)-C(69) & 0.1 & 0.2 & 0.2 & 0.1 \\
\hline 63 & BOND $\mathrm{H}(6)-\mathrm{C}(13)$ & 0.2 & 0.0 & 0.3 & 0.2 \\
\hline 64 & BOND H(34)-C(42) & -0.3 & -0.1 & -1.2 & -0.5 \\
\hline 65 & BOND C(13)-C(15) & 0.2 & 0.5 & 0.4 & 0.4 \\
\hline 66 & BOND C(69)-C(58) & 0.0 & -0.2 & -0.1 & -0.1 \\
\hline 67 & BOND H(75)-C(69) & -0.2 & 0.2 & -0.2 & 0.0 \\
\hline 68 & LP O(40) & 0.1 & 0.3 & 0.1 & 0.2 \\
\hline 69 & BOND C(77)-C(66) & 0.0 & -0.1 & 0.0 & 0.0 \\
\hline 70 & BOND H(10)-C(24) & 0.0 & 0.0 & 0.0 & 0.0 \\
\hline 71 & BOND H(21)-C(19) & -0.5 & -0.1 & -0.1 & -0.2 \\
\hline 72 & BOND C(80)-C(73) & -0.1 & -0.1 & -0.1 & -0.1 \\
\hline 73 & BOND C(20)-C(35) & 0.0 & 0.0 & 0.1 & 0.0 \\
\hline 74 & BOND C(42)-C(51) & 0.5 & 0.8 & -3.3 & -0.7 \\
\hline 75 & BOND H(38)-C(35) & -0.1 & -0.2 & 0.0 & -0.1 \\
\hline 76 & BOND H(52)-C(58) & -0.7 & 1.4 & 0.7 & 0.5 \\
\hline 77 & BOND H(78)-C(77) & 0.1 & 0.0 & 0.0 & 0.0 \\
\hline 78 & BOND C(24)-C(14) & 0.0 & 0.0 & 0.0 & 0.0 \\
\hline 79 & BOND H(57)-C(58) & -2.4 & 0.3 & -0.4 & -0.8 \\
\hline 80 & BOND H(1)-C(5) & 0.0 & 0.1 & 0.0 & 0.0 \\
\hline 81 & BOND H(18)-C(15) & -1.9 & -0.1 & 0.1 & -0.6 \\
\hline 82 & BOND H(8)-C(19) & -0.1 & 0.1 & 0.0 & 0.0 \\
\hline 83 & BOND C(20)-C(27) & 0.0 & 0.0 & 0.0 & 0.0 \\
\hline 84 & BOND H(64)-C(66) & 0.0 & 0.0 & 0.1 & 0.0 \\
\hline 85 & BOND O(40)-C(27) & 0.0 & 0.1 & 0.1 & 0.1 \\
\hline 86 & BOND O(62)-C(66) & 0.1 & 0.0 & 0.1 & 0.1 \\
\hline 87 & BOND H(39)-C(48) & -0.1 & 0.1 & 0.0 & 0.0 \\
\hline 88 & BOND O(32)-C(19) & 0.0 & -0.1 & 0.2 & 0.0 \\
\hline 89 & BOND C(48)-C(59) & 0.0 & 0.4 & -0.2 & 0.0 \\
\hline 90 & BOND C(42)-C(51) & -0.6 & -1.3 & -6.3 & -2.7 \\
\hline 91 & LP N(55) & -18.3 & 2.1 & -28.3 & -14.8 \\
\hline 92 & BOND C(5)-C(23) & -0.9 & -0.9 & -1.0 & -0.9 \\
\hline 93 & BOND N(55)-C(58) & -3.5 & 2.0 & -4.2 & -1.9 \\
\hline 94 & BOND C(67)-C(63) & -0.1 & -1.0 & -0.8 & -0.6 \\
\hline 95 & BOND $\mathrm{H}(9)-\mathrm{C}(13)$ & 0.3 & 0.0 & 0.0 & 0.1 \\
\hline 96 & BOND C(77)-C(80) & 0.0 & 0.0 & 0.0 & 0.0 \\
\hline 97 & BOND H(2)-C(5) & 0.0 & 0.2 & 0.0 & 0.1 \\
\hline 98 & BOND H(54)-C(47) & -0.1 & -0.1 & 0.1 & 0.0 \\
\hline 99 & BOND C(63)-C(51) & -0.7 & -3.8 & -3.7 & -2.8 \\
\hline 100 & BOND H(41)-C(33) & -0.1 & 0.0 & 0.1 & 0.0 \\
\hline 101 & BOND H(76)-C(73) & 0.1 & 0.0 & 0.0 & 0.0 \\
\hline 102 & BOND C(35)-C(47) & 0.0 & 0.0 & 0.0 & 0.0 \\
\hline 103 & BOND C(14)-C(19) & -0.1 & 0.0 & -0.1 & -0.1 \\
\hline 104 & BOND H(25)-C(27) & 0.0 & 0.0 & 0.0 & 0.0 \\
\hline 105 & BOND H(26)-C(13) & 0.3 & -0.3 & -0.3 & -0.1 \\
\hline 106 & BOND H(49)-C(45) & 0.0 & 0.1 & 0.1 & 0.1 \\
\hline 107 & LP O(62) & -0.1 & 0.0 & 0.1 & 0.0 \\
\hline 108 & BOND O(32)-C(33) & 0.1 & 0.1 & 0.1 & 0.1 \\
\hline 109 & BOND C(33)-C(24) & 0.0 & 0.0 & 0.0 & 0.0 \\
\hline 110 & BOND O(62)-C(73) & -0.1 & -0.1 & 0.2 & 0.0 \\
\hline 111 & LP N(29) & -3.5 & 2.5 & -16.5 & -5.8 \\
\hline 112 & BOND H(12)-C(20) & -0.1 & -0.1 & 0.1 & 0.0 \\
\hline 113 & BOND H(37)-C(45) & 0.0 & -0.1 & 0.0 & -0.1 \\
\hline 114 & BOND C(51)-Si(43) & -54.2 & -165.8 & -135.1 & -118.4 \\
\hline 115 & BOND C(45)-C(56) & 0.1 & -0.4 & 0.6 & 0.1 \\
\hline 116 & BOND H(53)-C(56) & -0.8 & -0.8 & -0.5 & -0.7 \\
\hline 117 & BOND N(29)-C(23) & -5.2 & -1.1 & -8.5 & -4.9 \\
\hline 118 & BOND N(55)-C(56) & -6.8 & -1.7 & -6.7 & -5.0 \\
\hline 119 & BOND N(29)-C(15) & -1.5 & 1.3 & -3.9 & -1.4 \\
\hline 120 & BOND H(74)-C(67) & -0.1 & 0.1 & 0.0 & 0.0 \\
\hline 121 & LP O $(40)$ & -0.4 & -0.6 & 0.1 & -0.3 \\
\hline 122 & BOND H(17)-C(23) & 0.4 & 0.3 & 0.0 & 0.2 \\
\hline 123 & LP O(62) & -0.1 & -0.2 & -0.1 & -0.1 \\
\hline
\end{tabular}




\begin{tabular}{lccccc}
124 & LP O(32) & -0.5 & -0.3 & 0.0 & -0.3 \\
125 & BOND C(48)-C(59) & 0.3 & 0.1 & -0.5 & 0.0 \\
126 & BOND C(67)-C(63) & 0.3 & -0.3 & -0.4 & -0.1 \\
127 & BOND C(48)-C(42) & 0.0 & -1.5 & -1.0 & -0.8 \\
128 & BOND N(55)-Si(43) & -87.8 & -41.8 & -98.9 & -76.2 \\
129 & BOND N(29)-Si(43) & -80.0 & -45.7 & -129.6 & -85.1 \\
130 & BOND Si(43)-Li(46) & -282.4 & -236.9 & 11.0 & -169.4 \\
\hline
\end{tabular}

Table 132. MO contributions to $\sigma$ in principal axes for Si in 6.3 THF [IGLO-BP86/mixed basis//B3LYP/TZVP].

\begin{tabular}{cccccc} 
MO & type & $\sigma_{1}$ & $\sigma_{2}$ & $\sigma_{3}$ & average \\
\hline 1 & AO Si(52) & 481.9 & 481.9 & 481.9 & 481.9 \\
2 & AO O(41) & 0.0 & 0.0 & 0.0 & 0.0 \\
3 & AO O(62) & 0.0 & 0.0 & 0.0 & 0.0 \\
4 & AO O(32) & 0.0 & 0.0 & 0.0 & 0.0 \\
5 & AO N(36) & 0.0 & 0.0 & 0.0 & 0.0 \\
6 & AO C(38) & 0.0 & 0.0 & 0.0 & 0.0 \\
7 & AO C(69) & 0.0 & 0.0 & 0.0 & 0.0 \\
8 & AO C(22) & 0.0 & 0.0 & 0.0 & 0.0 \\
9 & AO C(39) & 0.0 & 0.0 & 0.0 & 0.0 \\
10 & AO C(65) & 0.0 & 0.0 & 0.0 & 0.0 \\
11 & AO C(29) & 0.0 & 0.0 & 0.0 & 0.0 \\
12 & AO C(35) & 0.0 & 0.0 & 0.0 & 0.0 \\
13 & AO C(40) & 0.0 & 0.0 & 0.0 & 0.0 \\
14 & AO C(11) & 0.0 & 0.0 & 0.0 & 0.0 \\
15 & AO C(81) & 0.0 & 0.0 & 0.0 & 0.0 \\
16 & AO C(79) & 0.0 & 0.0 & 0.0 & 0.0 \\
17 & AO C(21) & 0.0 & 0.0 & 0.0 & 0.0 \\
18 & AO C(15) & 0.0 & 0.0 & 0.0 & 0.0 \\
19 & AO C(34) & 0.0 & 0.0 & 0.0 & 0.0 \\
20 & AO C(7) & 0.0 & 0.0 & 0.0 & 0.0 \\
21 & AO C(4) & 0.0 & 0.0 & 0.0 & 0.0 \\
22 & AO C(3) & 0.0 & 0.0 & 0.0 & 0.0 \\
23 & AO C(78) & 0.0 & 0.0 & 0.0 & 0.0 \\
24 & AO C(75) & 0.0 & 0.0 & 0.0 & 0.0 \\
25 & AO C(71) & 0.0 & 0.0 & 0.0 & 0.0 \\
26 & AO C(17) & 0.0 & 0.0 & 0.0 & 0.0 \\
27 & AO C(60) & 0.0 & 0.0 & 0.0 & 0.0 \\
28 & AO C (10) & 0.0 & 0.0 & 0.0 & 0.0 \\
29 & AO C(67) & 0.0 & 0.0 & 0.0 & 0.0 \\
30 & AO C(58) & 0.0 & 0.0 & 0.0 & 0.0 \\
31 & AO C(68) & 0.0 & 0.0 & 0.0 & 0.0 \\
32 & AO C(19) & 0.0 & 0.0 & 0.0 & 0.0 \\
33 & AO C(56) & 0.0 & 0.0 & 0.0 & 0.0 \\
34 & AO C(43) & 0.0 & 0.0 & 0.0 & 0.0 \\
35 & AO C(73) & 0.0 & 0.0 & 0.0 & 0.0 \\
36 & AO C(31) & 0.0 & 0.0 & 0.0 & 0.0 \\
37 & AO C(54) & 0.0 & 0.0 & 0.0 & 0.0 \\
38 & AO C(20) & 0.0 & 0.0 & 0.0 & 0.0 \\
39 & AO C(61) & 0.0 & -0.1 & 0.0 & 0.0 \\
40 & AO C(59) & 0.0 & 0.0 & -0.1 & 0.0 \\
41 & AO C(47) & 0.0 & 0.0 & 0.0 & 0.0 \\
42 & AO Si(52) & 78.0 & 79.8 & 72.3 & 76.7 \\
43 & AO Si(52) & 77.6 & 79.2 & 71.5 & 76.1 \\
44 & AO Si(52) & 76.7 & 62.5 & 86.0 & 75.1 \\
45 & AO Si(52) & 66.3 & 71.9 & 88.0 & 75.4 \\
46 & AO Li(49) & -0.1 & -0.1 & 0.1 & 0.0 \\
47 & BOND H(42)-C(35) & 0.0 & 0.0 & 0.0 & 0.0 \\
48 & BOND H(6)-C(11) & 0.0 & 0.0 & 0.0 & 0.0
\end{tabular}




\begin{tabular}{|c|c|c|c|c|c|}
\hline 49 & BOND H(84)-C(81) & 0.0 & 0.0 & 0.0 & 0.0 \\
\hline 50 & BOND H(2)-C(4) & 0.1 & 0.0 & 0.0 & 0.0 \\
\hline 51 & BOND C(10)-C(4) & 0.0 & 0.0 & 0.0 & 0.0 \\
\hline 52 & BOND C $(56)-C(61)$ & 1.2 & 0.0 & -3.5 & -0.8 \\
\hline 53 & BOND H(72)-C(67) & 0.0 & 0.1 & 0.1 & 0.1 \\
\hline 54 & BOND H(24)-C(35) & 0.0 & 0.0 & 0.0 & 0.0 \\
\hline 55 & BOND C (31)-C(19) & 0.1 & 0.1 & 0.5 & 0.2 \\
\hline 56 & BOND H(83)-C(79) & 0.0 & 0.0 & 0.1 & 0.0 \\
\hline 57 & BOND H(16)-C(15) & 0.0 & 0.1 & 0.0 & 0.0 \\
\hline 58 & BOND O(41)-C(38) & 0.2 & 0.1 & 0.1 & 0.1 \\
\hline 59 & BOND H(66)-C(69) & -0.1 & 0.1 & 0.0 & 0.0 \\
\hline 60 & BOND H(37)-C(29) & 0.1 & 0.0 & 0.0 & 0.0 \\
\hline 61 & BOND H(1)-C(3) & 0.0 & 0.0 & 0.0 & 0.0 \\
\hline 62 & BOND H(23)-C(17) & 0.0 & 0.2 & 0.0 & 0.1 \\
\hline 63 & BOND C(75)-C(68) & -0.2 & -0.9 & 0.4 & -0.2 \\
\hline 64 & BOND H(76)-C(71) & 0.1 & 0.1 & 0.1 & 0.1 \\
\hline 65 & BOND C (68)-C(59) & 0.5 & 0.2 & -2.0 & -0.4 \\
\hline 66 & BOND H(85)-C(78) & -0.1 & 0.1 & 0.0 & 0.0 \\
\hline 67 & BOND H(27)-C(31) & 0.1 & 0.0 & 0.0 & 0.0 \\
\hline 68 & BOND C (47)-C(43) & -0.2 & -0.4 & 0.4 & 0.0 \\
\hline 69 & BOND H(50)-C(40) & 0.0 & 0.0 & 0.0 & 0.0 \\
\hline 70 & BOND H(28)-C(39) & 0.1 & 0.0 & 0.0 & 0.0 \\
\hline 71 & BOND H(86)-C(81) & -0.1 & 0.0 & 0.0 & 0.0 \\
\hline 72 & BOND H(18)-C(22) & 0.0 & 0.0 & 0.1 & 0.0 \\
\hline 73 & BOND H(64)-C(65) & 0.0 & -0.1 & -0.1 & 0.0 \\
\hline 74 & BOND H(13)-C(11) & 0.0 & 0.0 & 0.0 & 0.0 \\
\hline 75 & BOND C(3)-C(7) & 0.3 & -0.1 & 0.5 & 0.2 \\
\hline 76 & BOND C(31)-C(43) & 0.0 & 0.0 & -0.1 & 0.0 \\
\hline 77 & BOND H(53)-C(58) & -0.1 & 0.1 & 0.0 & 0.0 \\
\hline 78 & BOND C(73)-C(78) & -1.1 & -0.6 & 0.2 & -0.5 \\
\hline 79 & BOND H(82)-C(75) & 0.0 & 0.0 & 0.0 & 0.0 \\
\hline 80 & BOND H(9)-C(19) & 0.0 & 0.0 & 0.0 & 0.0 \\
\hline 81 & BOND H(57)-C(60) & -0.1 & 0.2 & 0.0 & 0.0 \\
\hline 82 & BOND C (20)-C(34) & -0.2 & -0.3 & 0.7 & 0.1 \\
\hline 83 & BOND H(30)-C(38) & -0.1 & 0.0 & 0.1 & 0.0 \\
\hline 84 & BOND H(70)-C(69) & -0.1 & -0.1 & 0.1 & 0.0 \\
\hline 85 & BOND H(8)-C(15) & 0.0 & 0.0 & 0.0 & 0.0 \\
\hline 86 & BOND O(62)-C(69) & 0.2 & 0.0 & 0.1 & 0.1 \\
\hline 87 & BOND C(35)-C(40) & 0.0 & -0.1 & 0.0 & 0.0 \\
\hline 88 & BOND H(5)-C(7) & 0.0 & 0.1 & 0.0 & 0.0 \\
\hline 89 & BOND O(32)-C(22) & 0.1 & 0.1 & 0.1 & 0.1 \\
\hline 90 & BOND C (71)-C(60) & -0.4 & 0.0 & -0.8 & -0.4 \\
\hline 91 & BOND C (58)-C(54) & 0.1 & -0.6 & -1.0 & -0.5 \\
\hline 92 & BOND C(20)-C(34) & -1.0 & -0.9 & 2.1 & 0.1 \\
\hline 93 & BOND H(33)-C(40) & 0.0 & 0.0 & 0.0 & 0.0 \\
\hline 94 & BOND C (4)-C(3) & 0.0 & -0.1 & 0.0 & 0.0 \\
\hline 95 & BOND C (81)-C(79) & 0.0 & 0.0 & 0.0 & 0.0 \\
\hline 96 & LP O(32) & -0.3 & -0.4 & -0.1 & -0.3 \\
\hline 97 & BOND C (7)-C(17) & 0.0 & 0.1 & -0.1 & 0.0 \\
\hline 98 & BOND O(62)-C(65) & 0.0 & 0.1 & 0.2 & 0.1 \\
\hline 99 & BOND H(26)-C(22) & 0.0 & 0.0 & 0.0 & 0.0 \\
\hline 100 & BOND C (10)-C(4) & -0.4 & 0.0 & -0.1 & -0.2 \\
\hline 101 & BOND C (40)-C(39) & 0.0 & -0.2 & 0.0 & -0.1 \\
\hline 102 & BOND H(45)-C(56) & 0.3 & -0.6 & -0.5 & -0.3 \\
\hline 103 & BOND O(41)-C(39) & -0.1 & 0.0 & 0.2 & 0.0 \\
\hline 104 & BOND N(36)-C(21) & -1.6 & 1.2 & -1.2 & -0.5 \\
\hline 105 & BOND H(44)-C(54) & -0.3 & -0.3 & -1.4 & -0.7 \\
\hline 106 & BOND C(69)-C(81) & -0.1 & 0.0 & 0.0 & 0.0 \\
\hline 107 & BOND C(11)-C(15) & 0.0 & 0.0 & 0.0 & 0.0 \\
\hline 108 & BOND C (68)-C(59) & -1.1 & -1.2 & -4.9 & -2.4 \\
\hline 109 & BOND C (71)-C(78) & -0.1 & -0.1 & -0.1 & -0.1 \\
\hline 110 & BOND C (10)-C(21) & 0.1 & 0.2 & 0.7 & 0.3 \\
\hline
\end{tabular}




\begin{tabular}{|c|c|c|c|c|c|}
\hline 111 & BOND C(34)-C(47) & -0.5 & -0.7 & -0.1 & -0.5 \\
\hline 112 & BOND H(12)-C(20) & 0.0 & 0.0 & -0.1 & 0.0 \\
\hline 113 & BOND C(67)-C(58) & -0.1 & 0.0 & -0.1 & -0.1 \\
\hline 114 & BOND C(61)-C(73) & -0.5 & -3.6 & -4.7 & -2.9 \\
\hline 15 & BOND C (20)-C(19) & -0.1 & 0.1 & -0.1 & 0.0 \\
\hline 16 & BOND H(46)-C(39) & -0.2 & -0.4 & 0.0 & -0.2 \\
\hline 17 & BOND H(80)-C(79) & 0.0 & 0.0 & 0.0 & 0.0 \\
\hline 118 & BOND C(38)-C(35) & -0.1 & -0.1 & 0.1 & 0.0 \\
\hline 19 & BOND C(79)-C(65) & 0.0 & 0.0 & -0.1 & 0.0 \\
\hline 20 & BOND C(71)-C(60) & 0.0 & 0.0 & 0.1 & 0.0 \\
\hline 21 & BOND C $(11)-C(22)$ & 0.0 & 0.0 & 0.0 & 0.0 \\
\hline 122 & BOND C(75)-C(67) & 0.3 & -0.1 & -0.4 & -0.1 \\
\hline 123 & BOND H(25)-C(29) & -0.1 & -0.1 & -0.1 & -0.1 \\
\hline 24 & LP O(41) & -0.2 & -0.2 & 0.1 & -0.1 \\
\hline 25 & BOND C(3)-C(7) & 0.1 & 0.0 & -0.1 & 0.0 \\
\hline 126 & LP O(41) & 0.0 & -0.2 & 0.1 & 0.0 \\
\hline 127 & BOND H(63)-C(65) & 0.1 & 0.0 & 0.0 & 0.0 \\
\hline 128 & BOND C(15)-C(29) & 0.0 & -0.1 & -0.1 & -0.1 \\
\hline 129 & BOND C(31)-C(19) & 0.3 & -0.1 & 0.4 & 0.2 \\
\hline 130 & LP O(62) & 0.4 & 0.3 & 0.1 & 0.2 \\
\hline 131 & BOND C(73)-C(78) & 0.7 & -0.6 & -0.6 & -0.2 \\
\hline 132 & BOND C(60)-C(56) & 0.2 & -1.8 & 0.0 & -0.5 \\
\hline 133 & BOND O(32)-C(29) & 0.0 & -0.1 & 0.2 & 0.0 \\
\hline 134 & BOND C (75)-C(67) & 0.0 & 0.6 & 0.3 & 0.3 \\
\hline 135 & BOND C (17)-C(21) & -4.3 & 0.0 & -0.9 & -1.7 \\
\hline 136 & BOND H(51)-C(43) & 0.0 & 0.0 & 0.0 & 0.0 \\
\hline 37 & BOND N(36)-Si(52) & -83.2 & -31.5 & -100.8 & -71.9 \\
\hline 138 & BOND H(55)-C(47) & 0.3 & -0.2 & 0.3 & 0.1 \\
\hline 139 & BOND H(74)-C(68) & -0.5 & -0.1 & 0.8 & 0.1 \\
\hline 140 & BOND C(59)-Si(52) & -85.1 & -77.5 & -170.1 & -110.9 \\
\hline 141 & BOND N(36)-C(34) & -0.9 & 1.6 & 7.9 & 2.9 \\
\hline 142 & BOND H(48)-C(38) & 0.0 & 0.0 & 0.1 & 0.0 \\
\hline 143 & LP O(62) & -0.6 & -0.5 & -0.1 & -0.4 \\
\hline 144 & LP O(32) & 0.1 & 0.1 & 0.1 & 0.1 \\
\hline 145 & BOND H(77)-C(73) & -0.3 & -0.6 & -0.3 & -0.4 \\
\hline 146 & BOND H(14)-C(10) & -0.1 & 0.1 & -0.1 & 0.0 \\
\hline 147 & BOND C (56)-C(61) & -2.0 & -2.6 & -8.2 & -4.3 \\
\hline 148 & BOND C (17)-C(21) & -0.9 & 1.3 & 1.0 & 0.5 \\
\hline 149 & BOND C(61)-Si(52) & -70.2 & -123.1 & -134.9 & -109.4 \\
\hline 150 & BOND C(58)-C(54) & -0.2 & -0.4 & -0.2 & -0.3 \\
\hline 151 & BOND C(59)-C(54) & -1.8 & -3.0 & -4.9 & -3.2 \\
\hline 152 & BOND C (47)-C(43) & 0.0 & 0.2 & 0.5 & 0.2 \\
\hline 153 & BOND N(36)-C(34) & -2.9 & -1.9 & -2.7 & -2.5 \\
\hline 154 & BOND Si(52)-Li(49) & -311.5 & -173.9 & 8.1 & -159.1 \\
\hline
\end{tabular}

Table 133. MO contributions to $\sigma$ in principal axes for $\mathrm{Si}$ in 4 [IGLO-BP86/BIII//B3LYP/6-31+G(d)].

\begin{tabular}{cccccc} 
MO & type & $\sigma_{1}$ & $\sigma_{2}$ & $\sigma_{3}$ & average \\
\hline 1 & AO Si(7) & 481.9 & 481.9 & 481.9 & 481.9 \\
2 & AO N(8) & 0.0 & 0.0 & 0.0 & 0.0 \\
3 & AO C(17) & 0.0 & 0.0 & 0.0 & 0.0 \\
4 & AO C(9) & 0.0 & 0.0 & 0.0 & 0.0 \\
5 & AO C(5) & 0.0 & 0.0 & 0.0 & 0.0 \\
6 & AO C(4) & 0.0 & 0.0 & 0.0 & 0.0 \\
7 & AO C(13) & 0.0 & 0.0 & 0.0 & 0.0 \\
8 & AO C(6) & 0.0 & 0.0 & 0.0 & 0.0 \\
9 & AO C(3) & 0.0 & 0.0 & 0.0 & 0.0 \\
10 & AO C(14) & 0.0 & 0.0 & 0.0 & 0.0 \\
11 & AO C (1) & 0.0 & 0.0 & 0.0 & 0.0 \\
12 & AO C (12) & 0.0 & 0.0 & 0.0 & 0.0
\end{tabular}




\begin{tabular}{|c|c|c|c|c|c|}
\hline 13 & AO C(15) & 0.0 & 0.0 & 0.0 & 0.0 \\
\hline 14 & AO C(16) & 0.0 & 0.0 & 0.0 & 0.0 \\
\hline 15 & $\mathrm{AO} C(2)$ & 0.0 & 0.0 & -0.1 & 0.0 \\
\hline 16 & AO C(18) & 0.0 & 0.0 & 0.0 & 0.0 \\
\hline 17 & AO C(11) & 0.0 & -0.1 & 0.0 & 0.0 \\
\hline 18 & AO C(10) & 0.0 & 0.0 & 0.0 & 0.0 \\
\hline 19 & AO Si(7) & 68.3 & 69.5 & 86.7 & 74.8 \\
\hline 20 & AO Si(7) & 83.3 & 82.3 & 65.2 & 76.9 \\
\hline 21 & $\mathrm{AO} \mathrm{Si(7)}$ & 76.0 & 70.1 & 80.5 & 75.5 \\
\hline 22 & AO Si(7) & 68.8 & 72.3 & 84.1 & 75.1 \\
\hline 23 & AO Li(19) & -0.1 & -0.1 & 0.1 & 0.0 \\
\hline 24 & BOND H(29)-C(10) & 0.3 & 0.2 & -0.1 & 0.1 \\
\hline 25 & BOND H(37)-C(5) & 0.0 & 0.0 & 0.1 & 0.0 \\
\hline 26 & BOND C(13)-C(12) & -0.6 & 0.0 & -0.1 & -0.2 \\
\hline 27 & BOND H(32)-C(18) & 0.3 & 0.2 & 0.3 & 0.2 \\
\hline 28 & BOND C(6)-C(1) & -0.4 & -0.7 & -0.9 & -0.7 \\
\hline 29 & BOND H(36)-C(4) & -0.1 & 0.0 & 0.2 & 0.0 \\
\hline 30 & BOND H(24)-C(16) & 0.0 & -0.4 & -1.0 & -0.5 \\
\hline 31 & BOND H(25)-C(15) & -0.1 & 0.2 & 0.0 & 0.0 \\
\hline 32 & BOND H(30)-C(10) & -0.1 & 0.0 & 0.0 & 0.0 \\
\hline 33 & BOND H(33)-C(18) & 0.0 & 0.1 & -0.3 & -0.1 \\
\hline 34 & BOND C (4)-C(3) & -0.1 & -0.6 & 0.0 & -0.2 \\
\hline 35 & BOND C(3)-C(2) & -1.7 & -2.6 & -1.4 & -1.9 \\
\hline 36 & BOND $\mathrm{H}(26)-\mathrm{C}(14)$ & 0.1 & 0.1 & 0.1 & 0.1 \\
\hline 37 & BOND H(28)-C(13) & 0.0 & 0.1 & 0.1 & 0.0 \\
\hline 38 & BOND H(21)-C(17) & -3.9 & 0.8 & -0.7 & -1.2 \\
\hline 39 & BOND H(38)-C(6) & 0.0 & -0.1 & -0.1 & 0.0 \\
\hline 40 & BOND H(22)-C(9) & 0.3 & 0.5 & 1.1 & 0.6 \\
\hline 41 & BOND $\mathrm{C}(11)-\mathrm{C}(12)$ & -1.1 & -2.4 & -4.5 & -2.7 \\
\hline 42 & BOND N(8)-C(9) & -5.5 & -0.6 & -4.1 & -3.4 \\
\hline 43 & BOND H(35)-C(3) & -1.1 & -2.7 & 0.0 & -1.2 \\
\hline 44 & BOND C (14)-C(13) & -0.1 & -0.1 & -0.1 & -0.1 \\
\hline 45 & BOND C (4)-C(5) & -0.2 & -0.2 & 0.0 & -0.1 \\
\hline 46 & BOND C(15)-C(14) & 0.2 & 0.5 & 0.1 & 0.3 \\
\hline 47 & BOND C (11)-C(16) & 0.1 & 1.7 & -3.7 & -0.7 \\
\hline 48 & BOND H(34)-C(18) & 0.1 & 0.2 & 0.0 & 0.1 \\
\hline 49 & BOND C (6)-C(5) & 0.2 & 0.3 & 0.7 & 0.4 \\
\hline 50 & BOND C (13)-C(12) & 0.6 & -0.4 & -0.5 & -0.1 \\
\hline 51 & BOND H(20)-C(17) & -2.5 & 0.3 & 0.1 & -0.7 \\
\hline 52 & BOND H(31)-C(10) & 0.1 & 0.0 & 0.1 & 0.1 \\
\hline 53 & BOND C(2)-C(1) & 0.0 & -2.9 & -1.2 & -1.4 \\
\hline 54 & BOND C(10)-C(9) & -0.6 & -0.5 & -0.9 & -0.7 \\
\hline 55 & BOND C (15)-C(16) & -0.1 & -1.4 & -0.6 & -0.7 \\
\hline 56 & BOND H(39)-C(1) & -0.4 & -0.7 & -0.5 & -0.5 \\
\hline 57 & LP N(8) & -9.2 & 8.7 & -9.3 & -3.3 \\
\hline 58 & BOND H(23)-C(9) & -0.4 & -0.6 & 0.1 & -0.3 \\
\hline 59 & BOND N(8)-C(17) & -4.7 & 1.8 & -3.1 & -2.0 \\
\hline 60 & BOND C(18)-C(17) & -0.3 & 1.6 & 0.0 & 0.4 \\
\hline 61 & BOND C (4)-C(3) & -0.2 & -1.0 & 0.2 & -0.3 \\
\hline 62 & BOND H(27)-C(12) & -0.5 & 0.2 & -1.2 & -0.5 \\
\hline 63 & BOND C (11)-C(16) & 0.1 & 0.6 & -6.0 & -1.8 \\
\hline 64 & BOND C (11)-Si(7) & -75.4 & -127.7 & -136.8 & -113.3 \\
\hline 65 & BOND C(15)-C(14) & 0.4 & 0.5 & -0.4 & 0.2 \\
\hline 66 & BOND C(6)-C(5) & 0.5 & -0.1 & 0.8 & 0.4 \\
\hline 67 & BOND C (2)-Si(7) & -66.5 & -96.2 & -163.4 & -108.7 \\
\hline 68 & BOND C(2)-C(1) & 0.9 & -3.3 & -2.5 & -1.6 \\
\hline 69 & BOND N(8)-Si(7) & -118.3 & -43.6 & -95.8 & -85.9 \\
\hline 70 & BOND Si(7)-Li(19) & -256.8 & -182.4 & -4.7 & -148.0 \\
\hline
\end{tabular}

Table 134. MO contributions to $\sigma$ in principal axes for Si in 5 [IGLO-BP86/BIII//B3LYP/6-31+G(d)]. 


\begin{tabular}{|c|c|c|c|c|c|}
\hline $\mathrm{MO}$ & type & $\sigma_{1}$ & $\sigma_{2}$ & $\sigma_{3}$ & average \\
\hline 1 & AO Si(7) & 481.9 & 481.9 & 481.9 & 481.9 \\
\hline 2 & $A O N(8)$ & 0.0 & 0.0 & 0.0 & 0.0 \\
\hline 3 & AO N(11) & 0.0 & 0.0 & 0.0 & 0.0 \\
\hline 4 & AO C(14) & 0.0 & 0.0 & 0.0 & 0.0 \\
\hline 5 & AO C (9) & 0.0 & 0.0 & 0.0 & 0.0 \\
\hline 6 & $\mathrm{AO} C(12)$ & 0.0 & 0.0 & 0.0 & 0.0 \\
\hline 7 & AO C(16) & 0.0 & 0.0 & 0.0 & 0.0 \\
\hline 8 & $\mathrm{AO} C(3)$ & 0.0 & 0.0 & 0.0 & 0.0 \\
\hline 9 & AO C(4) & 0.0 & 0.0 & 0.0 & 0.0 \\
\hline 10 & $\mathrm{AO} C(2)$ & 0.0 & 0.0 & 0.0 & 0.0 \\
\hline 11 & AO C (5) & 0.0 & 0.0 & 0.0 & 0.0 \\
\hline 12 & AO C (1) & 0.0 & 0.0 & 0.0 & 0.0 \\
\hline 13 & AO C(15) & 0.0 & 0.0 & 0.0 & 0.0 \\
\hline 14 & AO C(17) & 0.0 & 0.0 & 0.0 & 0.0 \\
\hline 15 & AO C(10) & 0.0 & 0.0 & 0.0 & 0.0 \\
\hline 16 & $\mathrm{AO} C(6)$ & 0.0 & -0.1 & 0.0 & 0.0 \\
\hline 17 & $\mathrm{AO} C(26)$ & 0.0 & 0.0 & 0.0 & 0.0 \\
\hline 18 & AO Si(7) & 64.4 & 75.1 & 84.2 & 74.5 \\
\hline 19 & AO Si(7) & 78.0 & 66.7 & 82.3 & 75.7 \\
\hline 20 & AO Si(7) & 84.2 & 84.0 & 62.4 & 76.8 \\
\hline 21 & AO Si(7) & 71.9 & 66.8 & 88.0 & 75.6 \\
\hline 22 & AO Li(18) & -0.1 & -0.1 & 0.1 & 0.0 \\
\hline 23 & BOND H(35)-C(10) & 0.2 & 0.1 & 0.4 & 0.2 \\
\hline 24 & BOND H(41)-C(26) & 0.5 & 0.1 & 0.2 & 0.3 \\
\hline 25 & BOND H(30)-C(1) & -0.4 & -0.6 & -1.2 & -0.7 \\
\hline 26 & BOND N(11)-Si(7) & -76.0 & -53.6 & -72.3 & -67.3 \\
\hline 27 & BOND H(32)-C(17) & 0.1 & 0.2 & 0.1 & 0.1 \\
\hline 28 & BOND H(31)-C(2) & -0.1 & 0.1 & 0.0 & 0.0 \\
\hline 29 & BOND H(24)-C(4) & -0.1 & 0.1 & 0.0 & 0.0 \\
\hline 30 & BOND H(19)-C(9) & -0.4 & -0.5 & -0.1 & -0.3 \\
\hline 31 & BOND H(40)-C(15) & -0.3 & 0.3 & -0.2 & -0.1 \\
\hline 32 & BOND N(11)-C(14) & -4.3 & 0.8 & -4.3 & -2.6 \\
\hline 33 & BOND H(13)-C(12) & 0.0 & -0.5 & -0.2 & -0.2 \\
\hline 34 & BOND C(4)-C(3) & 0.4 & 0.7 & -0.5 & 0.2 \\
\hline 35 & BOND H(29)-C(3) & 0.1 & 0.1 & 0.1 & 0.1 \\
\hline 36 & BOND H(33)-C(17) & -0.1 & 0.1 & -0.1 & 0.0 \\
\hline 37 & BOND H(25)-C(12) & 0.0 & -0.9 & -0.1 & -0.3 \\
\hline 38 & BOND H(27)-C(14) & -1.5 & 1.5 & 0.1 & 0.0 \\
\hline 39 & BOND C $(6)-C(1)$ & -0.9 & -4.2 & -3.3 & -2.8 \\
\hline 40 & BOND C (17)-C(16) & -0.8 & -0.1 & -0.9 & -0.6 \\
\hline 41 & BOND H(28)-C(14) & -2.8 & -0.7 & -0.5 & -1.3 \\
\hline 42 & BOND H(34)-C(17) & 0.0 & 0.2 & 0.0 & 0.1 \\
\hline 43 & BOND C(6)-C(5) & -0.4 & 1.1 & -6.1 & -1.8 \\
\hline 44 & BOND C(3)-C(2) & -0.2 & 0.0 & -0.1 & -0.1 \\
\hline 45 & BOND H(39)-C(15) & 0.1 & 0.1 & 0.4 & 0.2 \\
\hline 46 & BOND H(38)-C(15) & -0.1 & 0.3 & -0.1 & 0.1 \\
\hline 47 & BOND H(37)-C(10) & 0.3 & 0.0 & 0.0 & 0.1 \\
\hline 48 & BOND H(21)-C(16) & 0.8 & 0.7 & -0.1 & 0.5 \\
\hline 49 & BOND H(23)-C(5) & -0.2 & -0.4 & -1.0 & -0.5 \\
\hline 50 & BOND H(36)-C(10) & 0.2 & -0.3 & -0.3 & -0.1 \\
\hline 51 & BOND C(2)-C(1) & 0.4 & -0.4 & -0.4 & -0.1 \\
\hline 52 & BOND C(15)-C(14) & 0.3 & -0.1 & 0.2 & 0.1 \\
\hline 53 & BOND N(8)-C(9) & -2.6 & 1.9 & -4.5 & -1.8 \\
\hline 54 & BOND H(43)-C(26) & 0.1 & 0.0 & 0.2 & 0.1 \\
\hline 55 & BOND H(42)-C(26) & 0.0 & -0.2 & 0.0 & -0.1 \\
\hline 56 & BOND N(8)-C(16) & -4.6 & 1.1 & -9.2 & -4.3 \\
\hline 57 & BOND C (4)-C(5) & 0.2 & -1.1 & -0.8 & -0.6 \\
\hline 58 & BOND H(20)-C(9) & -2.9 & -0.4 & 0.2 & -1.0 \\
\hline 59 & LP N(8) & -1.1 & 2.8 & -24.6 & -7.6 \\
\hline 60 & BOND C(26)-C(12) & 0.5 & -0.5 & 1.0 & 0.3 \\
\hline
\end{tabular}




\begin{tabular}{cccccc}
61 & BOND N(11)-C(12) & -5.1 & -2.6 & -5.2 & -4.3 \\
62 & BOND C(6)-Si(7) & -45.7 & -172.9 & -132.0 & -116.9 \\
63 & BOND C(4)-C(3) & 0.1 & 0.9 & -0.1 & 0.3 \\
64 & BOND C(10)-C(9) & -0.1 & 0.8 & 0.6 & 0.4 \\
65 & BOND H(22)-C(16) & -0.2 & 0.0 & -1.4 & -0.5 \\
66 & BOND C(6)-C(5) & 0.7 & 0.4 & -2.7 & -0.5 \\
67 & BOND C(2)-C(1) & -0.2 & -0.3 & -0.6 & -0.4 \\
68 & BOND N(11)-Si(7) & -27.8 & -6.1 & -43.6 & -25.8 \\
69 & BOND Si(7)-Li(18) & -252.0 & -206.2 & -2.5 & -153.6 \\
70 & BOND N(8)-Si(7) & -93.7 & -31.8 & -131.5 & -85.7 \\
\hline
\end{tabular}

Table 135. MO contributions to $\sigma$ in principal axes for Si in 6 [IGLO-BP86/BIII//B3LYP/6-31+G(d)].

\begin{tabular}{cccccc} 
MO & type & $\sigma_{1}$ & $\sigma_{2}$ & $\sigma_{3}$ & average \\
\hline 1 & AO Si(7) & 481.9 & 481.9 & 481.9 & 481.9 \\
2 & AO N(14) & 0.0 & 0.0 & 0.0 & 0.0 \\
3 & AO C(15) & 0.0 & 0.0 & 0.0 & 0.0 \\
4 & AO C(21) & 0.0 & 0.0 & 0.0 & 0.0 \\
5 & AO C(19) & 0.0 & 0.0 & 0.0 & 0.0 \\
6 & AO C(17) & 0.0 & 0.0 & 0.0 & 0.0 \\
7 & AO C(20) & 0.0 & 0.0 & 0.0 & 0.0 \\
8 & AO C(18) & 0.0 & 0.0 & 0.0 & 0.0 \\
9 & AO C(16) & 0.0 & 0.0 & 0.0 & 0.0 \\
10 & AO C(10) & 0.0 & 0.0 & 0.0 & 0.0 \\
11 & AO C(11) & 0.0 & 0.0 & 0.0 & 0.0 \\
12 & AO C(25) & 0.0 & 0.0 & 0.0 & 0.0 \\
13 & AO C(13) & 0.0 & 0.0 & 0.0 & 0.0 \\
14 & AO C(12) & 0.0 & 0.0 & 0.0 & 0.0 \\
15 & AO C(23) & 0.0 & 0.0 & 0.0 & 0.0 \\
16 & AO C(6) & 0.0 & 0.0 & 0.0 & 0.0 \\
17 & AO C(9) & 0.0 & 0.0 & 0.0 & 0.0 \\
18 & AO C(1) & 0.0 & 0.0 & 0.0 & 0.0 \\
19 & AO C(5) & 0.0 & 0.0 & 0.0 & 0.0 \\
20 & AO C(4) & 0.0 & 0.0 & 0.0 & 0.0 \\
21 & AO C(26) & 0.0 & 0.0 & 0.0 & 0.0 \\
22 & AO C(3) & 0.0 & 0.0 & 0.0 & 0.0 \\
23 & AO C(24) & 0.0 & 0.0 & 0.0 & 0.0 \\
24 & AO C(8) & 0.0 & -0.1 & -0.1 & 0.0 \\
25 & AO C(22) & 0.0 & 0.0 & 0.0 & 0.0 \\
26 & AO C(2) & 0.0 & 0.0 & -0.1 & 0.0 \\
27 & AO Si(7) & 77.3 & 80.2 & 72.5 & 76.7 \\
28 & AO Si(7) & 75.1 & 77.5 & 69.9 & 74.2 \\
29 & AO Si(7) & 75.6 & 63.1 & 85.3 & 74.7 \\
30 & AO Si(7) & 67.0 & 70.2 & 88.8 & 75.3 \\
31 & AO Li(27) & -0.1 & 0.0 & 0.1 & 0.0 \\
32 & BOND C(18)-C(19) & -0.3 & 0.0 & -0.1 & -0.1 \\
33 & BOND N(14)-Si(7) & -64.2 & -22.5 & -108.2 & -65.0 \\
34 & BOND C(10)-C(9) & 0.5 & -0.5 & -0.8 & -0.3 \\
35 & BOND C(2)-C(3) & -0.1 & -0.7 & -4.4 & -1.7 \\
36 & BOND H(30)-C(24) & 0.1 & 0.1 & 0.0 & 0.1 \\
37 & BOND H(37)-C(20) & 0.1 & 0.1 & 0.1 & 0.1 \\
38 & BOND H(41)-C(12) & -0.1 & 0.1 & 0.0 & 0.0 \\
39 & BOND C(4)-C(5) & 0.5 & 0.4 & -0.1 & 0.2 \\
40 & BOND C(12)-C(11) & -0.2 & 0.6 & -0.5 & 0.0 \\
41 & BOND H(32)-C(26) & 0.1 & 0.0 & -0.1 & 0.0 \\
42 & BOND C(6)-C(5) & 0.0 & -0.1 & 0.0 & -0.1 \\
43 & BOND H(45)-C(5) & 0.0 & 0.1 & 0.1 & 0.1 \\
44 & BOND H(31)-C(25) & 0.0 & 0.0 & 0.0 & 0.0 \\
45 & BOND H(35)-C(18) & 0.0 & 0.0 & 0.0 & 0.0 \\
46 & BOND C(20)-C(19) & 0.1 & 0.1 & -0.1 & 0.1 \\
& & & & &
\end{tabular}




\begin{tabular}{|c|c|c|c|c|c|}
\hline 47 & BOND C(26)-C(25) & 0.0 & 0.0 & 0.0 & 0.0 \\
\hline 48 & BOND C(11)-C(10) & -0.1 & -0.1 & -0.1 & -0.1 \\
\hline 49 & BOND C(8)-C(13) & 1.8 & -1.2 & -1.5 & -0.3 \\
\hline 50 & BOND C(4)-C(3) & -1.0 & -0.7 & -1.1 & -0.9 \\
\hline 51 & BOND C (12)-C(13) & 0.5 & -1.5 & 0.4 & -0.2 \\
\hline 52 & BOND H(36)-C(19) & 0.0 & 0.1 & 0.0 & 0.0 \\
\hline 53 & BOND H(34)-C(17) & 0.1 & 0.0 & 0.0 & 0.1 \\
\hline 54 & BOND H(46)-C(6) & 0.1 & -0.1 & 0.1 & 0.0 \\
\hline 55 & BOND C(24)-C(25) & 0.0 & 0.3 & 0.4 & 0.2 \\
\hline 56 & BOND $C(6)-C(1)$ & -0.7 & 0.3 & 0.4 & 0.0 \\
\hline 57 & BOND C(16)-C(17) & -0.8 & 0.0 & -0.5 & -0.4 \\
\hline 58 & BOND H(29)-C(23) & 0.0 & 0.0 & 0.0 & 0.0 \\
\hline 59 & BOND N(14)-C(21) & -1.2 & -1.6 & -3.4 & -2.0 \\
\hline 60 & BOND H(33)-C(16) & 0.0 & 0.1 & -0.1 & 0.0 \\
\hline 61 & BOND C(18)-C(17) & 0.0 & -0.1 & 0.0 & 0.0 \\
\hline 62 & BOND H(38)-C(9) & -0.9 & -1.0 & -0.7 & -0.9 \\
\hline 63 & BOND H(39)-C(10) & 0.0 & 0.0 & 0.0 & 0.0 \\
\hline 64 & BOND C $(10)-C(9)$ & -0.6 & -0.8 & 0.2 & -0.4 \\
\hline 65 & BOND C(8)-C(13) & -2.6 & -0.6 & -6.4 & -3.2 \\
\hline 66 & BOND C(24)-C(25) & 0.3 & -0.1 & 0.5 & 0.2 \\
\hline 67 & BOND C(4)-C(5) & 0.2 & 0.2 & 0.2 & 0.2 \\
\hline 68 & BOND C(18)-C(19) & 0.1 & 0.0 & 0.6 & 0.2 \\
\hline 69 & BOND H(40)-C(11) & 0.1 & 0.1 & 0.1 & 0.1 \\
\hline 70 & BOND C(20)-C(15) & -1.3 & 1.0 & 1.5 & 0.4 \\
\hline 71 & BOND C(21)-C(22) & -0.6 & -0.7 & -0.2 & -0.5 \\
\hline 72 & BOND C(20)-C(15) & -7.2 & -0.5 & -1.0 & -2.9 \\
\hline 73 & BOND H(42)-C(13) & 0.6 & -1.6 & -0.1 & -0.3 \\
\hline 74 & BOND H(47)-C(1) & -0.5 & 0.3 & 0.5 & 0.1 \\
\hline 75 & BOND C(26)-C(21) & 0.8 & -0.4 & 0.8 & 0.4 \\
\hline 76 & BOND C(15)-C(16) & 0.1 & 0.1 & 0.9 & 0.4 \\
\hline 77 & BOND C(12)-C(11) & 0.5 & 0.5 & 0.7 & 0.6 \\
\hline 78 & BOND C (2)-C(1) & -1.8 & -1.3 & -4.1 & -2.4 \\
\hline 79 & BOND C $(24)-C(23)$ & 0.0 & 0.0 & -0.1 & 0.0 \\
\hline 80 & BOND C (8)-Si(7) & -59.4 & -145.5 & -139.6 & -114.8 \\
\hline 81 & BOND C(8)-C(9) & -1.1 & -4.1 & -3.8 & -3.0 \\
\hline 82 & BOND H(44)-C(4) & -0.1 & 0.1 & 0.0 & 0.0 \\
\hline 83 & BOND C(22)-C(23) & -0.2 & 0.2 & 0.5 & 0.2 \\
\hline 84 & BOND N(14)-C(21) & -0.3 & 0.6 & 9.3 & 3.2 \\
\hline 85 & BOND C(16)-C(17) & -0.3 & 0.1 & 0.1 & -0.1 \\
\hline 86 & BOND N(14)-C(15) & -1.1 & 0.9 & -1.3 & -0.5 \\
\hline 87 & BOND C(26)-C(21) & -1.5 & -0.8 & 1.6 & -0.2 \\
\hline 88 & BOND H(43)-C(3) & -0.3 & -0.2 & -1.3 & -0.6 \\
\hline 89 & BOND C (2)-C(3) & -0.9 & 2.0 & -4.4 & -1.1 \\
\hline 90 & BOND C(22)-C(23) & 0.0 & -0.2 & 0.5 & 0.1 \\
\hline 91 & BOND C(6)-C(1) & 0.3 & 0.1 & 0.0 & 0.1 \\
\hline 92 & BOND H(28)-C(22) & -0.1 & 0.1 & 0.3 & 0.1 \\
\hline 93 & BOND C(2)-Si(7) & -111.4 & -66.9 & -177.3 & -118.5 \\
\hline 94 & BOND Si(7)-Li(27) & -312.7 & -185.3 & 8.0 & -163.3 \\
\hline
\end{tabular}

Table 136. MO contributions to $\sigma$ in principal axes for $\mathrm{Si}$ in $\mathrm{Me}_{3} \mathrm{SiH}$ [IGLO-BP86/BIII//B3LYP/6$31+G(d)]$.

\begin{tabular}{cccccc} 
MO & type & $\sigma_{1}$ & $\sigma_{2}$ & $\sigma_{3}$ & average \\
\hline 1 & AO Si(2) & 481.9 & 481.9 & 481.9 & 481.9 \\
2 & AO C(1) & 0.0 & 0.0 & 0.1 & 0.0 \\
3 & AO C(3) & 0.0 & 0.0 & 0.0 & 0.0 \\
4 & AO C(5) & 0.0 & 0.1 & 0.0 & 0.0 \\
5 & AO Si(2) & 76.0 & 83.7 & 67.3 & 75.7 \\
6 & AO Si(2) & 82.8 & 65.3 & 80.5 & 76.2 \\
7 & AO Si(2) & 64.1 & 79.6 & 80.8 & 74.8
\end{tabular}




\begin{tabular}{cccccc}
8 & AO Si(2) & 80.1 & 74.4 & 74.3 & 76.3 \\
9 & BOND H(7)-C(1) & -0.8 & -1.1 & 0.7 & -0.4 \\
10 & BOND H(12)-C(3) & -3.0 & 0.8 & 1.4 & -0.3 \\
11 & BOND H(9)-C(5) & -0.8 & 2.6 & -3.0 & -0.4 \\
12 & BOND H(4)-Si(2) & 7.6 & -136.0 & -135.0 & -87.8 \\
13 & BOND H(11)-C(5) & -3.0 & 2.9 & -0.7 & -0.3 \\
14 & BOND H(10)-C(5) & -0.8 & 1.5 & -1.9 & -0.4 \\
15 & BOND H(8)-C(1) & -0.8 & -3.1 & 2.7 & -0.4 \\
16 & BOND H(6)-C(1) & -3.0 & -0.4 & 2.6 & -0.3 \\
17 & BOND H(13)-C(3) & -0.8 & -2.1 & 1.7 & -0.4 \\
18 & BOND H(14)-C(3) & -0.8 & 1.0 & -1.4 & -0.4 \\
19 & BOND C(1)-Si(2) & -154.1 & -162.0 & -27.4 & -114.5 \\
20 & BOND C(5)-Si(2) & -152.8 & -14.7 & -176.1 & -114.6 \\
21 & BOND C(3)-Si(2) & -153.8 & -108.2 & -82.4 & -114.8 \\
\hline
\end{tabular}

Table 137. MO contributions to $\sigma$ in principal axes for $\mathrm{Si}$ in $\mathrm{Me}_{3} \mathrm{SiCl}$ [IGLO-BP86/BIII//B3LYP/6$31+G(d)]$.

\begin{tabular}{cccccc}
$\mathrm{MO}$ & type & $\sigma_{1}$ & $\sigma_{2}$ & $\sigma_{3}$ & average \\
\hline 1 & $\mathrm{AO} \mathrm{Cl(2)}$ & 0.0 & 0.0 & 0.0 & 0.0 \\
2 & $\mathrm{AO} \mathrm{Si}(1)$ & 481.9 & 481.9 & 481.9 & 481.9 \\
3 & $\mathrm{AO} \mathrm{C}(3)$ & 0.0 & 0.1 & 0.0 & 0.0 \\
4 & $\mathrm{AO} \mathrm{C}(4)$ & 0.1 & 0.0 & 0.0 & 0.0 \\
5 & $\mathrm{AO} \mathrm{C}(5)$ & 0.0 & 0.0 & 0.0 & 0.0 \\
6 & $\mathrm{AO} \mathrm{Cl}(2)$ & -0.2 & -0.3 & 0.1 & -0.1 \\
7 & $\mathrm{AO} \mathrm{Cl}(2)$ & 0.0 & 0.1 & 0.1 & 0.1 \\
8 & $\mathrm{AO} \mathrm{Cl}(2)$ & -0.3 & -0.3 & 0.1 & -0.2 \\
9 & $\mathrm{AO} \mathrm{Cl}(2)$ & 0.3 & 0.3 & 0.1 & 0.2 \\
10 & $\mathrm{AO} \mathrm{Si}(1)$ & 78.2 & 74.1 & 80.2 & 77.5 \\
11 & $\mathrm{AO} \mathrm{Si}(1)$ & 82.5 & 73.0 & 72.3 & 75.9 \\
12 & $\mathrm{AO} \mathrm{Si}(1)$ & 80.2 & 75.7 & 65.1 & 73.7 \\
13 & $\mathrm{AO} \mathrm{Si}(1)$ & 66.4 & 85.8 & 80.3 & 77.5 \\
14 & $\mathrm{LP} \mathrm{Cl}(2)$ & -3.4 & -2.7 & 7.3 & 0.4 \\
15 & BOND H(8)-C(3) & -3.9 & 3.5 & -0.9 & -0.5 \\
16 & BOND H(9)-C(4) & 3.5 & -3.9 & -0.9 & -0.5 \\
17 & BOND H(12)-C(5) & 0.5 & 0.0 & -2.5 & -0.7 \\
18 & BOND H(10)-C(4) & 1.2 & -1.7 & -0.9 & -0.5 \\
19 & BOND H(6)-C(3) & -3.0 & 3.5 & -2.5 & -0.7 \\
20 & BOND H(7)-C(3) & -2.2 & 1.7 & -0.9 & -0.5 \\
21 & BOND H(13)-C(5) & -2.0 & 1.5 & -0.9 & -0.5 \\
22 & BOND H(14)-C(5) & 2.0 & -2.5 & -0.9 & -0.5 \\
23 & BOND C(4)-Si(1) & -25.5 & -226.9 & -160.2 & -137.5 \\
24 & BOND H(11)-C(4) & 3.3 & -2.7 & -2.5 & -0.7 \\
25 & BOND Cl(2)-Si(1) & -119.8 & -119.7 & 5.3 & -78.1 \\
26 & LP Cl(2) & -5.3 & -0.9 & 7.3 & 0.4 \\
27 & LP Cl(2) & -0.6 & -5.7 & 7.3 & 0.3 \\
28 & BOND C(3)-Si(1) & -234.7 & -16.6 & -160.7 & -137.3 \\
29 & BOND C(5)-Si(1) & -117.1 & -135.3 & -159.9 & -137.4 \\
\hline
\end{tabular}

Table 138. MO contributions to $\sigma$ in principal axes for $\mathrm{Si}$ in $\mathrm{Me}_{3} \mathrm{SiLi}$ [IGLO-BP86/BIII//B3LYP/6$31+G(d)]$.

\begin{tabular}{cccccc} 
MO & type & $\sigma_{1}$ & $\sigma_{2}$ & $\sigma_{3}$ & average \\
\hline 1 & AO Si(1) & 481.9 & 481.9 & 481.9 & 481.9 \\
2 & AO C(4) & 0.0 & 0.0 & 0.0 & 0.0 \\
3 & AO C(3) & 0.1 & 0.0 & 0.0 & 0.0 \\
4 & AO C(5) & 0.0 & 0.0 & 0.0 & 0.0 \\
5 & AO Si(1) & 64.6 & 75.3 & 81.8 & 73.9 \\
6 & AO Si(1) & 83.2 & 83.2 & 62.1 & 76.2
\end{tabular}




\begin{tabular}{cccccc}
7 & AO Si(1) & 70.8 & 67.6 & 83.2 & 73.9 \\
8 & AO Si(1) & 73.8 & 66.5 & 81.3 & 73.9 \\
9 & AO Li(2) & -0.1 & -0.1 & 0.2 & 0.0 \\
10 & BOND H(10)-C(4) & 0.8 & -1.5 & -0.9 & -0.6 \\
11 & BOND C(3)-Si(1) & -21.1 & -155.8 & -144.1 & -107.0 \\
12 & BOND H(7)-C(3) & 2.6 & -3.3 & -1.0 & -0.6 \\
13 & BOND C(5)-Si(1) & -147.4 & -31.0 & -142.8 & -107.0 \\
14 & BOND H(12)-C(5) & 0.4 & 2.3 & -2.9 & -0.1 \\
15 & BOND H(14)-C(5) & -3.3 & 2.6 & -0.9 & -0.6 \\
16 & BOND H(8)-C(3) & 1.5 & -2.2 & -1.0 & -0.6 \\
17 & BOND H(6)-C(3) & 2.5 & 0.3 & -2.9 & -0.1 \\
18 & BOND H(9)-C(4) & -2.1 & 1.4 & -1.0 & -0.5 \\
19 & BOND H(13)-C(5) & -1.5 & 0.9 & -1.0 & -0.6 \\
20 & BOND H(11)-C(4) & 1.2 & 1.5 & -2.9 & -0.1 \\
21 & BOND C (4)-Si(1) & -97.4 & -79.0 & -144.4 & -106.9 \\
22 & BOND Si(1)-Li(2) & -175.5 & -175.6 & 10.6 & -113.5 \\
\hline
\end{tabular}

Table 139. MO contributions to $\sigma$ in principal axes for $\mathrm{Si}$ in $\mathrm{Me}_{4} \mathrm{Si}$ [IGLO-BP86/BIII//B3LYP/6$31+G(d)]$.

\begin{tabular}{cccccc} 
MO & type & $\sigma_{1}$ & $\sigma_{2}$ & $\sigma_{3}$ & average \\
\hline 1 & AO Si(1) & 481.9 & 481.9 & 481.9 & 481.9 \\
2 & AO C(5) & 0.0 & 0.0 & 0.1 & 0.0 \\
3 & AO C(2) & 0.0 & 0.0 & 0.0 & 0.0 \\
4 & AO C(4) & 0.0 & 0.0 & 0.0 & 0.0 \\
5 & AO C(3) & 0.1 & 0.0 & 0.0 & 0.0 \\
6 & AO Si(1) & 68.3 & 77.2 & 81.8 & 75.8 \\
7 & AO Si(1) & 83.4 & 74.5 & 69.9 & 75.9 \\
8 & AO Si(1) & 81.7 & 77.4 & 69.3 & 76.1 \\
9 & AO Si(1) & 71.0 & 74.7 & 82.3 & 76.0 \\
10 & BOND H(6)-C(2) & -3.0 & 0.9 & 0.8 & -0.4 \\
11 & BOND H(15)-C(5) & -1.2 & -1.3 & 1.2 & -0.4 \\
12 & BOND H(9)-C(3) & 2.9 & -2.4 & -1.8 & -0.4 \\
13 & BOND H(12)-C(4) & 0.1 & 1.4 & -2.7 & -0.4 \\
14 & BOND C(2)-Si(1) & -160.3 & -78.9 & -96.8 & -112.0 \\
15 & BOND H(13)-C(4) & 0.2 & 0.2 & -1.7 & -0.4 \\
16 & BOND H(16)-C(5) & -1.5 & -2.3 & 2.6 & -0.4 \\
17 & BOND H(11)-C(3) & 2.0 & -1.8 & -1.5 & -0.4 \\
18 & BOND C(5)-Si(1) & -165.5 & -155.1 & -16.3 & -112.3 \\
19 & BOND H(10)-C(3) & 3.0 & -1.5 & -2.9 & -0.4 \\
20 & BOND H(17)-C(5) & -2.9 & -1.2 & 2.9 & -0.4 \\
21 & BOND H(8)-C(2) & -1.1 & 1.6 & -1.7 & -0.4 \\
22 & BOND H(14)-C(4) & -2.4 & 2.3 & -1.2 & -0.4 \\
23 & BOND H(7)-C(2) & -1.1 & -1.0 & 0.8 & -0.4 \\
24 & BOND C(3)-Si(1) & -0.9 & -164.5 & -170.5 & -112.0 \\
25 & BOND C(4)-Si(1) & -122.5 & -49.9 & -164.3 & -112.2 \\
\hline
\end{tabular}

Table 140. MO contributions to $\sigma$ in principal axes for $\mathrm{Si}$ in (MeO)Me ${ }_{2} \mathrm{SiH}$ [IGLO-BP86/BIII//B3LYP/6$31+G(d)]$.

\begin{tabular}{cccccc} 
MO & type & $\sigma_{1}$ & $\sigma_{2}$ & $\sigma_{3}$ & average \\
\hline 1 & AO Si(1) & 481.9 & 481.9 & 481.9 & 481.9 \\
2 & AO O(3) & 0.0 & 0.0 & 0.1 & 0.0 \\
3 & AO C(5) & 0.0 & 0.0 & 0.0 & 0.0 \\
4 & AO C(4) & 0.0 & 0.0 & 0.0 & 0.0 \\
5 & AO C(2) & 0.0 & 0.0 & 0.0 & 0.0 \\
6 & AO Si(1) & 78.7 & 88.0 & 69.2 & 78.6 \\
7 & AO Si(1) & 87.5 & 62.9 & 83.4 & 78.0 \\
8 & AO Si(1) & 82.1 & 80.9 & 68.9 & 77.3
\end{tabular}




\begin{tabular}{cccccc}
9 & AO Si(1) & 64.0 & 86.6 & 79.5 & 76.7 \\
10 & BOND H(6)-Si(1) & -0.7 & -193.7 & -128.1 & -107.5 \\
11 & BOND H(10)-C(4) & -3.1 & 2.4 & -0.5 & -0.4 \\
12 & LP O(3) & -0.6 & 7.8 & 13.5 & 6.9 \\
13 & BOND H(8)-C(2) & -0.9 & -0.9 & 0.3 & -0.5 \\
14 & BOND H(14)-C(5) & -0.2 & -3.0 & 1.4 & -0.6 \\
15 & BOND H(15)-C(5) & -0.7 & -0.5 & 1.5 & 0.1 \\
16 & BOND H(13)-C(5) & -1.2 & -2.9 & 0.2 & -1.3 \\
17 & BOND O(3)-Si(1) & -137.1 & -146.8 & 0.1 & -94.6 \\
18 & BOND H(11)-C(4) & 0.1 & -0.3 & -1.1 & -0.4 \\
19 & BOND H(12)-C(4) & -1.4 & 1.5 & -2.7 & -0.9 \\
20 & BOND H(9)-C(2) & -3.3 & 1.8 & 0.3 & -0.4 \\
21 & BOND H(7)-C(2) & -3.1 & 2.3 & -2.6 & -1.1 \\
22 & BOND C(4)-Si(1) & -170.8 & -69.5 & -168.9 & -136.4 \\
23 & BOND C(2)-Si(1) & -207.5 & -69.1 & -135.4 & -137.3 \\
24 & LP O(3) & 8.5 & 1.5 & 14.5 & 8.2 \\
25 & BOND O(3)-C(5) & -3.6 & -8.5 & 4.3 & -2.6 \\
\hline
\end{tabular}

Table 141. MO contributions to $\sigma$ in principal axes for $\mathrm{Si}$ in (MeO)Me ${ }_{2} \mathrm{SiCl}$ [IGLO-BP86/BIII//B3LYP/6$31+G(d)]$.

\begin{tabular}{cccccc} 
MO & type & $\sigma_{1}$ & $\sigma_{2}$ & $\sigma_{3}$ & average \\
\hline 1 & $\mathrm{AO} \mathrm{Cl(3)}$ & 0.0 & 0.0 & 0.0 & 0.0 \\
2 & $\mathrm{AO} \mathrm{Si}(1)$ & 481.9 & 481.9 & 481.9 & 481.9 \\
3 & $\mathrm{AO} \mathrm{O}(4)$ & 0.0 & 0.1 & 0.0 & 0.0 \\
4 & $\mathrm{AO} \mathrm{C}(6)$ & 0.0 & 0.0 & 0.0 & 0.0 \\
5 & $\mathrm{AO} \mathrm{C}(2)$ & 0.0 & 0.0 & 0.0 & 0.0 \\
6 & $\mathrm{AO} \mathrm{C}(5)$ & 0.0 & 0.0 & 0.1 & 0.0 \\
7 & $\mathrm{AO} \mathrm{Cl}(3)$ & 0.1 & -0.2 & -0.3 & -0.1 \\
8 & $\mathrm{AO} \mathrm{Cl(3)}$ & 0.0 & 0.1 & 0.1 & 0.1 \\
9 & $\mathrm{AO} \mathrm{Cl(3)}$ & 0.1 & -0.4 & -0.3 & -0.2 \\
10 & $\mathrm{AO} \mathrm{Cl(3)}$ & 0.1 & 0.3 & 0.3 & 0.2 \\
11 & $\mathrm{AO} \mathrm{Si(1)}$ & 74.6 & 74.3 & 87.5 & 78.8 \\
12 & $\mathrm{AO} \mathrm{Si(1)}$ & 69.9 & 82.4 & 79.8 & 77.4 \\
13 & $\mathrm{AO} \mathrm{Si(1)}$ & 86.7 & 87.6 & 65.5 & 79.9 \\
14 & $\mathrm{AO} \mathrm{Si}(1)$ & 79.1 & 64.9 & 90.0 & 78.0 \\
15 & BOND H(13)-C(6) & -1.5 & 0.1 & -2.2 & -1.2 \\
16 & $\mathrm{LP} \mathrm{Cl(3)}$ & 7.7 & -4.6 & -7.4 & -1.4 \\
17 & BOND H(7)-C(2) & -1.6 & -2.7 & 1.2 & -1.0 \\
18 & $\mathrm{LP} \mathrm{O}(4)$ & 8.7 & 12.0 & 3.6 & 8.1 \\
19 & BOND H(10)-C(5) & -2.7 & -2.7 & 3.0 & -0.8 \\
20 & BOND H(15)-C(6) & -0.4 & 0.6 & -0.2 & 0.0 \\
21 & BOND O(4)-C(6) & -4.7 & 5.0 & -13.8 & -4.5 \\
22 & BOND H(14)-C(6) & -1.0 & 0.9 & 0.1 & 0.0 \\
23 & BOND C(2)-Si(1) & -172.8 & -142.5 & -129.1 & -148.1 \\
24 & BOND H(12)-C(5) & -3.5 & -2.8 & 2.4 & -1.3 \\
25 & BOND H(11)-C(5) & -1.0 & -2.1 & 1.5 & -0.5 \\
26 & BOND H(9)-C(2) & -2.8 & 0.6 & 0.0 & -0.7 \\
27 & BOND H(8)-C(2) & 0.1 & 1.2 & -3.0 & -0.5 \\
28 & LP Cl(3) & 5.9 & -2.8 & -2.7 & 0.1 \\
29 & BOND C(5)-Si(1) & -211.5 & -227.4 & -9.7 & -149.5 \\
30 & BOND Cl(3)-Si(1) & -2.3 & -117.6 & -154.3 & -91.4 \\
31 & LP Cl(3) & 7.5 & 0.8 & -5.9 & 0.8 \\
32 & BOND O(4)-Si(1) & -136.7 & -2.0 & -153.2 & -97.3 \\
33 & LP O(4) & 0.1 & 12.6 & -1.7 & 3.7 \\
\hline & & & & &
\end{tabular}

Table 142. MO contributions to $\sigma$ in principal axes for $\mathrm{Si}$ in (MeO)Me ${ }_{2} \mathrm{SiLi}$ [IGLO-BP86/BIII//B3LYP/6$31+G(d)]$. 


\begin{tabular}{cccccc} 
MO & type & $\sigma_{1}$ & $\sigma_{2}$ & $\sigma_{3}$ & average \\
\hline 1 & AO Si(1) & 481.9 & 481.9 & 481.9 & 481.9 \\
2 & AO O(4) & 0.0 & 0.0 & 0.1 & 0.0 \\
3 & AO C(6) & 0.0 & 0.0 & 0.0 & 0.0 \\
4 & AO C(5) & 0.0 & 0.0 & 0.0 & 0.0 \\
5 & AO C(2) & 0.0 & 0.0 & 0.0 & 0.0 \\
6 & AO Si(1) & 81.6 & 74.1 & 73.2 & 76.3 \\
7 & AO Si(1) & 77.9 & 72.7 & 78.3 & 76.3 \\
8 & AO Si(1) & 67.1 & 87.7 & 73.0 & 75.9 \\
9 & AO Si(1) & 77.4 & 82.3 & 63.6 & 74.4 \\
10 & AO Li(3) & -0.1 & 0.1 & 0.0 & 0.0 \\
11 & BOND H(15)-C(6) & -4.5 & 0.2 & 1.3 & -1.0 \\
12 & BOND H(7)-C(2) & 2.6 & -2.8 & -2.7 & -1.0 \\
13 & BOND C(5)-Si(1) & -91.1 & -161.3 & -149.7 & -134.0 \\
14 & BOND H(10)-C(5) & 2.1 & -2.3 & -0.4 & -0.2 \\
15 & BOND O(4)-C(6) & -6.3 & -3.8 & 4.5 & -1.9 \\
16 & BOND H(13)-C(6) & -5.0 & -1.0 & 0.2 & -1.9 \\
17 & BOND H(14)-C(6) & -0.2 & -0.7 & 1.4 & 0.1 \\
18 & LP O(4) & -2.7 & 6.8 & 13.6 & 5.9 \\
19 & BOND H(9)-C(2) & 2.7 & -2.8 & -0.6 & -0.2 \\
20 & BOND H(8)-C(2) & 0.1 & -0.9 & -1.2 & -0.7 \\
21 & BOND H(11)-C(5) & -0.8 & -0.2 & -0.9 & -0.6 \\
22 & BOND H(12)-C(5) & 1.6 & -2.4 & -2.7 & -1.2 \\
23 & BOND C(2)-Si(1) & -61.8 & -186.8 & -154.8 & -134.5 \\
24 & LP O(4) & 5.3 & 4.3 & 12.2 & 7.3 \\
25 & BOND O(4)-Si(1) & -140.4 & -135.0 & 5.4 & -90.0 \\
26 & BOND Si(1)-Li(3) & -306.1 & -15.4 & -143.4 & -155.0 \\
\hline
\end{tabular}

Table 143. MO contributions to $\sigma$ in principal axes for $\mathrm{Si}$ in (MeO)Me ${ }_{2} \mathrm{SiMe}$ [IGLOBP86/BIII//B3LYP/6-31+G(d)].

\begin{tabular}{cccccc} 
MO & type & $\sigma_{1}$ & $\sigma_{2}$ & $\sigma_{3}$ & average \\
\hline 1 & AO Si(1) & 481.9 & 481.9 & 481.9 & 481.9 \\
2 & AO O(3) & 0.0 & 0.0 & 0.1 & 0.0 \\
3 & AO C(5) & 0.0 & 0.0 & 0.0 & 0.0 \\
4 & AO C(6) & 0.0 & 0.0 & 0.0 & 0.0 \\
5 & AO C(2) & 0.0 & 0.0 & 0.0 & 0.0 \\
6 & AO C(4) & 0.0 & 0.1 & 0.0 & 0.0 \\
7 & AO Si(1) & 85.9 & 72.0 & 77.6 & 78.5 \\
8 & AO Si(1) & 62.9 & 88.0 & 80.0 & 77.0 \\
9 & AO Si(1) & 86.6 & 70.9 & 75.4 & 77.6 \\
10 & AO Si(1) & 81.3 & 85.4 & 65.7 & 77.5 \\
11 & BOND H(14)-C(5) & -0.7 & -0.8 & 1.1 & -0.1 \\
12 & BOND H(9)-C(2) & -0.7 & 0.1 & -1.1 & -0.6 \\
13 & LP O(3) & 3.1 & 5.8 & 12.8 & 7.3 \\
14 & BOND H(16)-C(6) & 1.9 & -2.4 & -1.1 & -0.5 \\
15 & BOND H(12)-C(4) & -4.5 & 3.2 & -2.6 & -1.3 \\
16 & LP O(3) & 1.7 & 7.2 & 13.0 & 7.3 \\
17 & BOND H(13)-C(5) & -1.5 & -1.9 & 0.2 & -1.1 \\
18 & BOND H(15)-C(5) & -0.6 & -1.1 & 1.2 & -0.2 \\
19 & BOND C(4)-Si(1) & -236.1 & -23.8 & -139.3 & -133.1 \\
20 & BOND C(6)-Si(1) & -63.9 & -170.2 & -156.7 & -130.3 \\
21 & BOND H(10)-C(4) & -2.7 & 1.2 & -0.2 & -0.6 \\
22 & BOND H(7)-C(2) & 1.5 & -2.1 & -2.5 & -1.0 \\
23 & BOND H(17)-C(6) & 1.9 & -2.3 & -2.5 & -1.0 \\
24 & BOND H(11)-C(4) & -2.3 & 1.2 & -0.3 & -0.5 \\
25 & BOND H(18)-C(6) & -0.5 & -0.1 & -1.0 & -0.5 \\
26 & BOND H(8)-C(2) & 2.0 & -2.2 & -1.2 & -0.5 \\
27 & BOND O(3)-C(5) & -2.7 & -9.6 & 4.3 & -2.7 \\
28 & BOND O(3)-Si(1) & -137.2 & -135.4 & 2.3 & -90.1
\end{tabular}


Table 144. MO contributions to $\sigma$ in principal axes for $\mathrm{Si}$ in $\left(\mathrm{Me}_{2} \mathrm{~N}\right) \mathrm{Me}_{2} \mathrm{SiH}$ [IGLO-BP86/BIII//B3LYP/6$31+G(d)]$.

\begin{tabular}{cccccc} 
MO & type & $\sigma_{1}$ & $\sigma_{2}$ & $\sigma_{3}$ & average \\
\hline 1 & AO Si(2) & 481.9 & 481.9 & 481.9 & 481.9 \\
2 & AO N(3) & 0.0 & 0.0 & 0.1 & 0.0 \\
3 & AO C(4) & 0.0 & 0.0 & 0.0 & 0.0 \\
4 & AO C(10) & 0.0 & 0.0 & 0.0 & 0.0 \\
5 & AO C(8) & 0.0 & 0.0 & 0.0 & 0.0 \\
6 & AO C(9) & 0.0 & 0.0 & 0.0 & 0.0 \\
7 & AO Si(2) & 76.5 & 80.9 & 74.6 & 77.3 \\
8 & AO Si(2) & 69.8 & 77.9 & 78.2 & 75.3 \\
9 & AO Si(2) & 82.2 & 71.1 & 77.4 & 76.9 \\
10 & AO Si(2) & 75.1 & 82.7 & 70.9 & 76.2 \\
11 & BOND H(1)-Si(2) & 6.9 & -167.6 & -130.7 & -97.1 \\
12 & BOND H(7)-C(4) & -0.1 & -1.2 & 0.3 & -0.3 \\
13 & BOND H(19)-C(8) & -2.1 & 2.1 & -2.1 & -0.7 \\
14 & BOND H(15)-C(9) & -2.1 & 2.2 & -2.1 & -0.7 \\
15 & BOND H(6)-C(4) & -0.8 & -0.5 & 0.5 & -0.3 \\
16 & BOND H(13)-C(10) & -0.8 & -0.5 & 0.5 & -0.3 \\
17 & BOND H(5)-C(4) & -0.1 & 0.3 & 0.9 & 0.3 \\
18 & BOND H(11)-C(10) & -0.1 & 0.3 & 0.9 & 0.3 \\
19 & BOND H(17)-C(8) & -3.2 & 1.8 & 0.0 & -0.5 \\
20 & BOND H(18)-C(8) & -0.8 & -0.7 & -0.5 & -0.7 \\
21 & BOND H(12)-C(10) & -0.1 & -1.2 & 0.3 & -0.3 \\
22 & BOND H(14)-C(9) & -3.2 & 1.8 & 0.0 & -0.5 \\
23 & BOND H(16)-C(9) & -0.8 & -0.7 & -0.5 & -0.7 \\
24 & BOND N(3)-C(4) & -2.3 & -6.4 & 3.7 & -1.6 \\
25 & BOND N(3)-C(10) & -2.3 & -6.5 & 3.7 & -1.7 \\
26 & LP N(3) & 0.6 & -8.5 & 10.9 & 1.0 \\
27 & BOND C(9)-Si(2) & -179.2 & -56.4 & -138.2 & -124.6 \\
28 & BOND C(8)-Si(2) & -177.9 & -55.8 & -137.8 & -123.8 \\
29 & BOND N(3)-Si(2) & -130.9 & -149.1 & -1.8 & -93.9 \\
\hline \multicolumn{7}{c}{} & & & &
\end{tabular}

Table 145. MO contributions to $\sigma$ in principal axes for $\mathrm{Si}$ in $\left(\mathrm{Me}_{2} \mathrm{~N}\right) \mathrm{Me}_{2} \mathrm{SiCl}$ [IGLO-BP86/BIII//B3LYP/6$31+G(d)]$.

\begin{tabular}{cccccc}
$\mathrm{MO}$ & type & $\sigma_{1}$ & $\sigma_{2}$ & $\sigma_{3}$ & average \\
\hline 1 & $\mathrm{AO} \mathrm{Cl}(1)$ & 0.0 & 0.0 & 0.0 & 0.0 \\
2 & $\mathrm{AO} \mathrm{Si}(2)$ & 481.9 & 481.9 & 481.9 & 481.9 \\
3 & $\mathrm{AO} \mathrm{N}(3)$ & 0.0 & 0.0 & 0.1 & 0.0 \\
4 & $\mathrm{AO} \mathrm{C}(10)$ & 0.0 & 0.0 & 0.0 & 0.0 \\
5 & $\mathrm{AO} \mathrm{C}(4)$ & 0.0 & 0.0 & 0.0 & 0.0 \\
6 & $\mathrm{AO} \mathrm{C}(9)$ & 0.0 & 0.0 & 0.0 & 0.0 \\
7 & $\mathrm{AO} \mathrm{C}(8)$ & 0.0 & 0.0 & 0.0 & 0.0 \\
8 & $\mathrm{AO} \mathrm{Cl}(1)$ & 0.1 & -0.2 & -0.2 & -0.1 \\
9 & $\mathrm{AO} \mathrm{Cl}(1)$ & 0.1 & 0.1 & 0.1 & 0.1 \\
10 & $\mathrm{AO} \mathrm{Cl(1)}$ & 0.1 & -0.4 & -0.4 & -0.2 \\
11 & $\mathrm{AO} \mathrm{Cl}(1)$ & 0.1 & 0.3 & 0.3 & 0.2 \\
12 & $\mathrm{AO} \mathrm{Si}(2)$ & 79.9 & 90.9 & 63.8 & 78.2 \\
13 & $\mathrm{AO} \mathrm{Si}(2)$ & 71.9 & 75.5 & 82.3 & 76.6 \\
14 & $\mathrm{AO} \mathrm{Si}(2)$ & 79.3 & 64.9 & 90.1 & 78.1 \\
15 & $\mathrm{AO} \mathrm{Si}(2)$ & 68.0 & 87.8 & 74.6 & 76.8 \\
16 & $\mathrm{BOND} \mathrm{H(13)-C(10)}$ & -0.7 & -0.7 & 0.4 & -0.4 \\
17 & $\mathrm{LP} \mathrm{Cl(1)}$ & 7.7 & -4.3 & -2.4 & 0.3 \\
18 & $\mathrm{BOND} \mathrm{H(5)-C(4)}$ & 0.1 & 0.9 & 1.0 & 0.7 \\
19 & $\mathrm{BOND} \mathrm{H(17)-C(8)}$ & -2.5 & 1.6 & -1.7 & -0.9
\end{tabular}




\begin{tabular}{lccccc}
20 & BOND H(14)-C(9) & -2.5 & 1.4 & -1.6 & -0.9 \\
21 & BOND H(6)-C(4) & -0.7 & -0.7 & 0.4 & -0.3 \\
22 & BOND N(3)-Si(2) & -115.7 & -161.6 & -8.5 & -95.3 \\
23 & BOND H(11)-C(10) & 0.1 & 0.9 & 1.0 & 0.7 \\
24 & BOND N(3)-C(4) & -1.5 & -9.7 & 4.2 & -2.3 \\
25 & BOND H(7)-C(4) & 0.0 & -0.4 & 0.3 & 0.0 \\
26 & BOND H(15)-C(9) & -2.4 & 2.6 & -2.4 & -0.7 \\
27 & BOND H(19)-C(8) & -2.4 & 2.7 & -2.5 & -0.8 \\
28 & BOND H(18)-C(8) & -1.2 & -0.9 & -0.4 & -0.8 \\
29 & BOND H(16)-C(9) & -1.3 & -1.1 & -0.2 & -0.9 \\
30 & BOND N(3)-C(10) & -1.5 & -9.8 & 4.3 & -2.3 \\
31 & BOND H(12)-C(10) & -0.1 & -0.3 & 0.3 & 0.0 \\
32 & LP Cl(1) & 7.4 & -5.4 & -1.3 & 0.2 \\
33 & LP Cl(1) & 6.6 & -4.8 & -4.2 & -0.8 \\
34 & BOND C(9)-Si(2) & -191.8 & -60.5 & -166.0 & -139.4 \\
35 & BOND C(8)-Si(2) & -191.8 & -53.6 & -173.1 & -139.5 \\
36 & BOND Cl(1)-Si(2) & 3.3 & -137.2 & -113.5 & -82.4 \\
37 & BOND N(3)-Si(2) & -2.2 & -39.6 & 8.8 & -11.0 \\
\hline
\end{tabular}

Table 146. MO contributions to $\sigma$ in principal axes for $\mathrm{Si}$ in $\left(\mathrm{Me}_{2} \mathrm{~N}\right) \mathrm{Me}_{2} \mathrm{SiLi}$ [IGLO-BP86/BIII//B3LYP/6$31+G(d)]$.

\begin{tabular}{cccccc} 
MO & type & $\sigma_{1}$ & $\sigma_{2}$ & $\sigma_{3}$ & average \\
\hline 1 & AO Si(2) & 481.9 & 481.9 & 481.9 & 481.9 \\
2 & AO N(3) & 0.0 & 0.0 & 0.1 & 0.0 \\
3 & AO C(10) & 0.0 & 0.0 & 0.0 & 0.0 \\
4 & AO C(4) & 0.0 & 0.0 & 0.0 & 0.0 \\
5 & AO C(8) & 0.0 & 0.0 & 0.0 & 0.0 \\
6 & AO C(9) & 0.0 & 0.0 & 0.0 & 0.0 \\
7 & AO Si(2) & 78.0 & 75.0 & 73.5 & 75.5 \\
8 & AO Si(2) & 78.2 & 68.7 & 79.3 & 75.4 \\
9 & AO Si(2) & 68.5 & 85.3 & 70.6 & 74.8 \\
10 & AO Si(2) & 75.3 & 79.9 & 65.8 & 73.7 \\
11 & AO Li(1) & -0.1 & 0.1 & -0.1 & 0.0 \\
12 & BOND N(3)-C(4) & -5.4 & -2.7 & 4.2 & -1.3 \\
13 & BOND N(3)-C(10) & -5.4 & -2.7 & 4.1 & -1.3 \\
14 & BOND H(19)-C(8) & 2.1 & -2.2 & -2.2 & -0.8 \\
15 & BOND H(15)-C(9) & 2.1 & -2.2 & -2.2 & -0.8 \\
16 & BOND H(7)-C(4) & -4.0 & 0.2 & 0.5 & -1.1 \\
17 & BOND H(13)-C(10) & -0.3 & -0.7 & 0.6 & -0.1 \\
18 & BOND H(5)-C(4) & -1.7 & -0.2 & 1.0 & -0.3 \\
19 & BOND H(11)-C(10) & -1.7 & -0.2 & 1.0 & -0.3 \\
20 & BOND H(6)-C(4) & -0.3 & -0.7 & 0.6 & -0.1 \\
21 & BOND H(17)-C(8) & 1.9 & -3.0 & 0.1 & -0.3 \\
22 & BOND H(16)-C(9) & -0.4 & -0.7 & -1.2 & -0.8 \\
23 & BOND H(12)-C(10) & -4.0 & 0.2 & 0.5 & -1.1 \\
24 & BOND H(18)-C(8) & -0.4 & -0.7 & -1.1 & -0.8 \\
25 & BOND H(14)-C(9) & 1.9 & -2.9 & 0.1 & -0.3 \\
26 & LP N(3) & 0.6 & 3.2 & 10.7 & 4.8 \\
27 & BOND C(9)-Si(2) & -59.7 & -164.5 & -136.5 & -120.2 \\
28 & BOND C(8)-Si(2) & -59.0 & -163.1 & -136.0 & -119.4 \\
29 & BOND Si(2)-Li(1) & -242.4 & 2.9 & -163.8 & -134.4 \\
30 & BOND N(3)-Si(2) & -182.3 & -134.8 & 4.2 & -104.3 \\
\hline & & & & & \\
\hline
\end{tabular}

Table 147. MO contributions to $\sigma$ in principal axes for $\mathrm{Si}$ in $\left(\mathrm{Me}_{2} \mathrm{~N}\right) \mathrm{Me}_{2} \mathrm{SiMe}$ [IGLOBP86/BIII//B3LYP/6-31+G(d)].

\begin{tabular}{cccccc} 
MO & type & $\sigma_{1}$ & $\sigma_{2}$ & $\sigma_{3}$ & average \\
\hline 1 & AO Si(2) & 481.9 & 481.9 & 481.9 & 481.9
\end{tabular}




\begin{tabular}{cccccc}
2 & AO N(3) & 0.0 & 0.0 & 0.1 & 0.0 \\
3 & AO C(4) & 0.0 & 0.0 & 0.0 & 0.0 \\
4 & AO C(10) & 0.0 & 0.0 & 0.0 & 0.0 \\
5 & AO C(9) & 0.0 & 0.0 & 0.0 & 0.0 \\
6 & AO C(1) & 0.0 & 0.0 & 0.0 & 0.0 \\
7 & AO C(8) & 0.1 & 0.0 & 0.0 & 0.0 \\
8 & AO Si(2) & 77.4 & 74.1 & 80.4 & 77.3 \\
9 & AO Si(2) & 65.9 & 82.2 & 80.7 & 76.3 \\
10 & AO Si(2) & 83.7 & 74.9 & 72.2 & 76.9 \\
11 & AO Si(2) & 78.0 & 82.1 & 70.7 & 76.9 \\
12 & BOND N(3)-Si(2) & -128.3 & -134.7 & -5.1 & -89.3 \\
13 & BOND H(15)-C(9) & -1.9 & 1.3 & -1.9 & -0.9 \\
14 & BOND H(7)-C(4) & -0.1 & 0.7 & 0.9 & 0.5 \\
15 & BOND H(20)-C(1) & -2.6 & 2.0 & -2.0 & -0.8 \\
16 & BOND H(17)-C(8) & 3.0 & -2.7 & -1.7 & -0.5 \\
17 & BOND H(12)-C(10) & 0.1 & 0.5 & 0.9 & 0.5 \\
18 & BOND H(5)-C(4) & -0.1 & -0.6 & 0.3 & -0.1 \\
19 & BOND H(13)-C(10) & -0.8 & -0.7 & 0.4 & -0.3 \\
20 & BOND H(6)-C(4) & -0.9 & -0.4 & 0.5 & -0.3 \\
21 & BOND H(18)-C(8) & 2.6 & -2.4 & -1.7 & -0.5 \\
22 & BOND H(21)-C(1) & -0.9 & -0.4 & -0.7 & -0.7 \\
23 & BOND H(22)-C(1) & -3.1 & 1.9 & -0.4 & -0.6 \\
24 & BOND C(1)-Si(2) & -184.4 & -46.6 & -134.8 & -121.9 \\
25 & BOND H(14)-C(9) & -0.6 & -0.8 & -0.5 & -0.6 \\
26 & BOND H(19)-C(8) & 3.2 & -3.0 & -2.5 & -0.8 \\
27 & BOND N(3)-C(10) & -2.2 & -6.3 & 3.2 & -1.8 \\
28 & BOND H(16)-C(9) & -3.0 & 1.4 & -0.3 & -0.6 \\
29 & BOND H(11)-C(10) & -0.5 & -0.3 & 0.2 & -0.2 \\
30 & BOND N(3)-C(4) & -2.5 & -6.0 & 3.2 & -1.8 \\
31 & BOND C(8)-Si(2) & 6.2 & -203.7 & -169.0 & -122.1 \\
32 & BOND C(9)-Si(2) & -167.2 & -67.6 & -129.1 & -121.3 \\
33 & LP N(3) & 0.9 & -10.0 & 12.9 & 1.3 \\
\hline & & & & & \\
\hline & & & & \\
\hline
\end{tabular}

Table 148. MO contributions to $\sigma$ in principal axes for $\mathrm{Si}$ in (MeS)Me ${ }_{2} \mathrm{SiH}$ [IGLO-BP86/BIII//B3LYP/6$31+G(d)]$.

\begin{tabular}{cccccc} 
MO & type & $\sigma_{1}$ & $\sigma_{2}$ & $\sigma_{3}$ & average \\
\hline 1 & AO S(3) & 0.0 & 0.0 & 0.0 & 0.0 \\
2 & AO Si(1) & 481.9 & 481.9 & 481.9 & 481.9 \\
3 & AO C(5) & 0.0 & 0.0 & 0.0 & 0.0 \\
4 & AO C(2) & 0.0 & 0.0 & 0.0 & 0.0 \\
5 & AO C(4) & 0.0 & 0.1 & 0.0 & 0.0 \\
6 & AO S(3) & -0.2 & -0.3 & 0.0 & -0.2 \\
7 & AO S(3) & -0.5 & -0.4 & 0.1 & -0.3 \\
8 & AO S(3) & 0.3 & 0.3 & 0.0 & 0.2 \\
9 & AO S(3) & 0.2 & 0.1 & 0.3 & 0.2 \\
10 & AO Si(1) & 76.6 & 82.4 & 67.3 & 75.4 \\
11 & AO Si(1) & 84.0 & 62.4 & 83.6 & 76.6 \\
12 & AO Si(1) & 77.9 & 77.5 & 71.2 & 75.6 \\
13 & AO Si(1) & 64.6 & 80.4 & 80.6 & 75.2 \\
14 & BOND C(5)-S(3) & -2.1 & -2.8 & 3.7 & -0.4 \\
15 & BOND H(11)-C(4) & -2.3 & 2.2 & -1.5 & -0.6 \\
16 & BOND H(8)-C(2) & 1.0 & -3.1 & 0.5 & -0.5 \\
17 & BOND H(13)-C(5) & -0.6 & -0.7 & 0.1 & -0.4 \\
18 & BOND H(6)-Si(1) & -14.8 & -149.3 & -130.0 & -98.0 \\
19 & BOND H(15)-C(5) & -0.6 & -0.3 & 0.7 & -0.1 \\
20 & BOND H(14)-C(5) & -0.3 & -2.0 & 0.8 & -0.5 \\
21 & BOND H(10)-C(4) & -3.5 & 3.1 & -0.7 & -0.4 \\
22 & BOND H(7)-C(2) & 1.2 & 0.3 & -2.1 & -0.2 \\
23 & BOND H(9)-C(2) & -2.1 & 0.3 & 0.5 & -0.4
\end{tabular}




\begin{tabular}{lccccc}
24 & BOND H(12)-C(4) & -2.1 & 4.0 & -2.5 & -0.2 \\
25 & LP S(3) & -8.8 & -3.1 & 7.5 & -1.5 \\
26 & BOND C(2)-Si(1) & -110.6 & -150.2 & -121.6 & -127.5 \\
27 & BOND C(4)-Si(1) & -208.1 & 0.8 & -174.5 & -127.3 \\
28 & BOND S(3)-Si(1) & -146.5 & -144.0 & -7.9 & -99.5 \\
29 & LP S(3) & -1.9 & -9.7 & 8.4 & -1.1 \\
\hline
\end{tabular}

Table 149. MO contributions to $\sigma$ in principal axes for $\mathrm{Si}$ in (MeS) $\mathrm{Me}_{2} \mathrm{SiCl}$ [IGLO-BP86/BIII//B3LYP/6$31+G(d)]$.

\begin{tabular}{|c|c|c|c|c|c|}
\hline $\mathrm{MO}$ & type & $\sigma_{1}$ & $\sigma_{2}$ & $\sigma_{3}$ & average \\
\hline 1 & $\mathrm{AO} \mathrm{Cl(3)}$ & 0.0 & 0.0 & 0.0 & 0.0 \\
\hline 2 & AO S(4) & 0.0 & 0.0 & 0.0 & 0.0 \\
\hline 3 & AO Si(1) & 481.9 & 481.9 & 481.9 & 481.9 \\
\hline 4 & $\mathrm{AO} C(6)$ & 0.0 & 0.0 & 0.0 & 0.0 \\
\hline 5 & AO C(5) & 0.0 & 0.0 & 0.0 & 0.0 \\
\hline 6 & $\mathrm{AO} C(2)$ & 0.0 & 0.0 & 0.0 & 0.0 \\
\hline 7 & $\mathrm{AO} \mathrm{Cl(3)}$ & -0.4 & -0.2 & 0.1 & -0.2 \\
\hline 8 & AO S(4) & 0.4 & 0.1 & 0.4 & 0.3 \\
\hline 9 & $\mathrm{AO} \mathrm{Cl(3)}$ & 0.2 & 0.2 & -0.1 & 0.1 \\
\hline 10 & $\mathrm{AO} \mathrm{Cl(3)}$ & -0.1 & -0.4 & 0.1 & -0.1 \\
\hline 11 & $\mathrm{AO} \mathrm{Cl(3)}$ & 0.2 & 0.3 & 0.2 & 0.2 \\
\hline 12 & AO S(4) & 0.0 & 0.2 & -0.1 & 0.1 \\
\hline 13 & AO S(4) & -0.3 & 0.2 & -0.1 & -0.1 \\
\hline 14 & AO S(4) & -0.3 & 0.0 & -0.4 & -0.3 \\
\hline 15 & AO Si(1) & 85.6 & 72.7 & 71.4 & 76.6 \\
\hline 16 & AO Si(1) & 80.0 & 80.9 & 67.0 & 75.9 \\
\hline 17 & AO Si(1) & 62.2 & 88.1 & 84.0 & 78.1 \\
\hline 18 & AO Si(1) & 83.4 & 66.9 & 76.6 & 75.6 \\
\hline 19 & LP Cl(3) & 0.8 & -5.2 & 7.0 & 0.9 \\
\hline 20 & LP S(4) & -7.3 & 6.4 & -2.0 & -0.9 \\
\hline 21 & BOND $\mathrm{H}(8)-C(2)$ & -1.1 & -2.5 & 1.6 & -0.7 \\
\hline 22 & BOND H(12)-C(5) & 3.9 & -2.3 & -2.0 & -0.1 \\
\hline 23 & BOND H(13)-C(6) & -1.9 & 0.0 & 0.8 & -0.4 \\
\hline 24 & BOND H(14)-C(6) & -0.4 & 0.7 & -0.6 & -0.1 \\
\hline 25 & BOND H(10)-C(5) & 1.1 & 0.2 & -3.0 & -0.6 \\
\hline 26 & BOND H(15)-C(6) & -0.5 & 0.6 & -0.5 & -0.1 \\
\hline 27 & BOND $\mathrm{H}(9)-\mathrm{C}(2)$ & 3.1 & -2.5 & -2.0 & -0.5 \\
\hline 28 & BOND Cl(3)-Si(1) & -126.8 & -106.1 & -32.2 & -88.4 \\
\hline 29 & LP Cl(3) & -2.7 & 0.4 & 2.3 & 0.0 \\
\hline 30 & BOND H(7)-C(2) & 1.9 & -2.5 & 0.2 & -0.1 \\
\hline 31 & LP Cl(3) & -7.2 & 0.2 & 6.6 & -0.1 \\
\hline 32 & BOND H(11)-C(5) & -0.1 & 0.3 & -1.9 & -0.6 \\
\hline 33 & BOND C(5)-Si(1) & -64.3 & -159.5 & -206.9 & -143.6 \\
\hline 34 & BOND C(2)-Si(1) & -96.1 & -226.5 & -106.1 & -142.9 \\
\hline 35 & BOND C (6)-S(4) & -4.1 & 4.5 & -2.1 & -0.6 \\
\hline 36 & BOND S(4)-Si(1) & -222.9 & -13.7 & -128.1 & -121.6 \\
\hline 37 & LP S(4) & -13.0 & 9.3 & -6.3 & -3.4 \\
\hline
\end{tabular}

Table 150. MO contributions to $\sigma$ in principal axes for Si in (MeS)Me ${ }_{2}$ SiLi [IGLO-BP86/BIII//B3LYP/6$31+G(d)]$.

\begin{tabular}{cccccc} 
MO & type & $\sigma_{1}$ & $\sigma_{2}$ & $\sigma_{3}$ & average \\
\hline 1 & AO S(4) & 0.0 & 0.0 & 0.0 & 0.0 \\
2 & AO Si(1) & 481.9 & 481.9 & 481.9 & 481.9 \\
3 & AO C(6) & 0.0 & 0.0 & 0.0 & 0.0 \\
4 & AO C(5) & 0.0 & 0.0 & 0.0 & 0.0 \\
5 & AO C(2) & 0.0 & 0.0 & 0.0 & 0.0 \\
6 & AO S(4) & -0.5 & -0.5 & 0.2 & -0.3
\end{tabular}




\begin{tabular}{cccccc}
7 & AO S(4) & 0.0 & 0.0 & 0.0 & 0.0 \\
8 & AO S(4) & 0.0 & 0.0 & 0.1 & 0.0 \\
9 & AO S(4) & 0.3 & 0.3 & 0.1 & 0.3 \\
10 & AO Si(1) & 74.4 & 73.4 & 71.7 & 73.2 \\
11 & AO Si(1) & 80.2 & 67.7 & 78.9 & 75.6 \\
12 & AO Si(1) & 63.3 & 87.3 & 74.1 & 74.9 \\
13 & AO Si(1) & 75.4 & 78.0 & 64.1 & 72.5 \\
14 & AO Li(3) & -0.1 & 0.1 & 0.0 & 0.0 \\
15 & BOND C(6)-S(4) & -2.3 & -1.1 & 2.7 & -0.2 \\
16 & BOND H(7)-C(2) & 1.9 & -0.7 & -2.3 & -0.4 \\
17 & BOND H(11)-C(5) & 0.1 & -1.5 & -1.1 & -0.8 \\
18 & BOND H(14)-C(6) & -0.1 & -0.5 & 0.5 & 0.0 \\
19 & LP S(4) & -3.7 & -6.7 & 7.1 & -1.1 \\
20 & BOND H(13)-C(6) & -3.9 & 0.4 & 0.1 & -1.1 \\
21 & BOND H(15)-C(6) & -3.2 & 0.0 & 0.7 & -0.8 \\
22 & BOND H(9)-C(2) & 1.4 & -2.1 & -1.3 & -0.7 \\
23 & BOND H(12)-C(5) & 2.6 & -1.4 & -2.2 & -0.3 \\
24 & BOND H(8)-C(2) & -1.0 & 0.2 & -1.4 & -0.7 \\
25 & BOND H(10)-C(5) & 2.1 & -3.4 & -0.8 & -0.7 \\
26 & BOND C(5)-Si(1) & -48.5 & -174.8 & -140.0 & -121.1 \\
27 & BOND C(2)-Si(1) & -97.7 & -123.9 & -156.4 & -126.0 \\
28 & BOND S(4)-Si(1) & -134.2 & -124.2 & 2.7 & -85.2 \\
29 & BOND Si(1)-Li(3) & -246.7 & -22.6 & -134.7 & -134.7 \\
30 & LP S(4) & -8.5 & -5.3 & 7.8 & -2.0 \\
\hline
\end{tabular}

Table 151. MO contributions to $\sigma$ in principal axes for $\mathrm{Si}$ in (MeS)Me ${ }_{2} \mathrm{SiMe}$ [IGLOBP86/BIII//B3LYP/6-31+G(d)].

\begin{tabular}{cccccc} 
MO & type & $\sigma_{1}$ & $\sigma_{2}$ & $\sigma_{3}$ & average \\
\hline 1 & AO S(3) & 0.0 & 0.0 & 0.0 & 0.0 \\
2 & AO Si(1) & 481.9 & 481.9 & 481.9 & 481.9 \\
3 & AO C(5) & 0.0 & 0.0 & 0.0 & 0.0 \\
4 & AO C(4) & 0.0 & 0.0 & 0.0 & 0.0 \\
5 & AO C(2) & 0.0 & 0.0 & 0.0 & 0.0 \\
6 & AO C(6) & 0.0 & 0.0 & 0.0 & 0.0 \\
7 & AO S(3) & 0.4 & 0.4 & 0.1 & 0.3 \\
8 & AO S(3) & 0.0 & 0.0 & 0.1 & 0.1 \\
9 & AO S(3) & -0.3 & -0.2 & 0.2 & -0.1 \\
10 & AO S(3) & -0.3 & -0.4 & 0.0 & -0.3 \\
11 & AO Si(1) & 74.4 & 74.3 & 79.5 & 76.1 \\
12 & AO Si(1) & 81.2 & 66.2 & 82.7 & 76.7 \\
13 & AO Si(1) & 68.9 & 81.1 & 77.7 & 75.9 \\
14 & AO Si(1) & 81.0 & 77.1 & 63.9 & 74.0 \\
15 & BOND C(5)-S(3) & -3.0 & -2.1 & 3.5 & -0.5 \\
16 & BOND H(12)-C(4) & 3.3 & -2.1 & -2.1 & -0.3 \\
17 & BOND H(7)-C(2) & -0.9 & 2.1 & -2.3 & -0.4 \\
18 & BOND H(18)-C(6) & 0.1 & -0.5 & -1.2 & -0.5 \\
19 & BOND H(14)-C(5) & -0.5 & -0.6 & 0.6 & -0.2 \\
20 & BOND H(11)-C(4) & 1.5 & -3.0 & -0.1 & -0.5 \\
21 & BOND H(13)-C(5) & -1.7 & 0.8 & 0.1 & -0.2 \\
22 & BOND H(15)-C(5) & -0.5 & -0.6 & 0.6 & -0.2 \\
23 & BOND H(8)-C(2) & -2.4 & 1.9 & -1.3 & -0.6 \\
24 & BOND C(2)-Si(1) & -150.2 & -57.7 & -163.1 & -123.6 \\
25 & BOND H(17)-C(6) & -0.9 & 2.1 & -2.3 & -0.4 \\
26 & BOND C(4)-Si(1) & -33.2 & -204.2 & -131.7 & -123.0 \\
27 & BOND H(16)-C(6) & -2.3 & 1.9 & -1.4 & -0.6 \\
28 & BOND H(9)-C(2) & 0.2 & -0.5 & -1.2 & -0.5 \\
29 & BOND S(3)-Si(1) & -155.5 & -122.7 & -2.7 & -93.6 \\
30 & BOND C(6)-Si(1) & -151.5 & -57.8 & -163.0 & -124.1 \\
31 & BOND H(10)-C(4) & 1.6 & -3.0 & -0.1 & -0.5
\end{tabular}




\begin{tabular}{llllll}
32 & LP S(3) & -4.8 & -6.8 & 6.8 & -1.6 \\
33 & LP S(3) & -4.6 & -6.8 & 6.8 & -1.5 \\
\hline
\end{tabular}

Table 152. $\mathrm{MO}$ contributions to $\sigma$ in principal axes for $\mathrm{Si}$ in (MeO) ${ }_{2} \mathrm{MeSiH}$ [IGLO-BP86/BIII//B3LYP/6$31+G(d)]$.

\begin{tabular}{|c|c|c|c|c|c|}
\hline $\mathrm{MO}$ & type & $\sigma_{1}$ & $\sigma_{2}$ & $\sigma_{3}$ & average \\
\hline 1 & $\mathrm{AO} \mathrm{Si(2)}$ & 481.9 & 481.9 & 481.9 & 481.9 \\
\hline 2 & $\mathrm{AO} O(4)$ & 0.0 & 0.0 & 0.0 & 0.0 \\
\hline 3 & $\mathrm{AO} O(3)$ & 0.0 & 0.0 & 0.0 & 0.0 \\
\hline 4 & $\mathrm{AO} C(9)$ & 0.0 & 0.0 & 0.0 & 0.0 \\
\hline 5 & AO C(13) & 0.0 & 0.0 & 0.0 & 0.0 \\
\hline 6 & $\mathrm{AO} C(5)$ & 0.0 & 0.0 & 0.1 & 0.0 \\
\hline 7 & AO Si(2) & 72.8 & 73.2 & 92.5 & 79.5 \\
\hline 8 & AO Si(2) & 86.6 & 85.6 & 66.1 & 79.4 \\
\hline 9 & AO Si(2) & 83.4 & 64.9 & 88.3 & 78.9 \\
\hline 10 & AO Si(2) & 77.7 & 78.3 & 83.7 & 79.9 \\
\hline 11 & BOND H(16)-C(13) & -0.3 & 0.4 & 0.3 & 0.1 \\
\hline 12 & OND H(10)-C(9) & -1.3 & -0.6 & -0.8 & -0.9 \\
\hline 13 & BOND O(3)-Si(2) & -129.6 & -35.0 & -145.3 & -103.3 \\
\hline 14 & BOND H(12)-C(9) & 0.6 & -0.1 & -0.8 & -0.1 \\
\hline 15 & LP O(3) & -1.8 & 11.2 & 12.3 & 7.3 \\
\hline 16 & LP O(4) & 13.2 & 13.6 & -2.1 & 8.3 \\
\hline 17 & BOND H(14)-C(13) & -0.9 & -0.6 & -1.8 & -1.1 \\
\hline 18 & $\mathrm{LP} O(3)$ & 7.3 & 4.0 & 8.0 & 6.4 \\
\hline 19 & BOND O(4)-C(9) & -7.0 & 1.3 & -3.9 & -3.2 \\
\hline 20 & BOND H(15)-C(13) & -0.4 & -0.6 & -0.9 & -0.6 \\
\hline 21 & BOND H(11)-C(9) & 0.4 & -0.4 & -0.5 & -0.2 \\
\hline 22 & BOND H(7)-C(5) & -4.1 & -1.7 & 1.6 & -1.4 \\
\hline 23 & ND H(6)-C(5) & -3.2 & -1.3 & 2.6 & -0.6 \\
\hline 24 & BOND $\mathrm{H}(8)-C(5)$ & -2.8 & -2.8 & 2.6 & -1.0 \\
\hline 25 & BOND C(5)-Si(2) & -229.0 & -214.5 & 4.2 & -146.5 \\
\hline 26 & BOND H(1)-Si(2) & -25.0 & -159.3 & -168.8 & -117.7 \\
\hline 27 & BOND O(4)-Si(2) & -107.2 & -53.1 & -146.9 & -102.4 \\
\hline 28 & BOND O(3)-C(13) & -2.4 & 2.9 & -9.7 & -3.1 \\
\hline 29 & LP O(4) & 9.4 & 0.8 & 8.2 & 6.1 \\
\hline
\end{tabular}

Table 153. MO contributions to $\sigma$ in principal axes for $\mathrm{Si}$ in (MeO) ${ }_{2} \mathrm{MeSiCl}$ [IGLO-BP86/BIII//B3LYP/6$31+G(d)]$.

\begin{tabular}{cccccc} 
MO & type & $\sigma_{1}$ & $\sigma_{2}$ & $\sigma_{3}$ & average \\
\hline 1 & $\mathrm{AO} \mathrm{Cl}(1)$ & 0.0 & 0.0 & 0.0 & 0.0 \\
2 & $\mathrm{AO} \mathrm{Si}(2)$ & 481.9 & 481.9 & 481.9 & 481.9 \\
3 & $\mathrm{AO} \mathrm{O}(4)$ & 0.0 & 0.0 & 0.0 & 0.0 \\
4 & $\mathrm{AO} \mathrm{O}(3)$ & 0.0 & 0.0 & 0.0 & 0.0 \\
5 & $\mathrm{AO} \mathrm{C}(13)$ & 0.0 & 0.0 & 0.0 & 0.0 \\
6 & $\mathrm{AO} \mathrm{C}(9)$ & 0.0 & 0.0 & 0.0 & 0.0 \\
7 & $\mathrm{AO} \mathrm{C}(5)$ & 0.0 & 0.0 & 0.1 & 0.0 \\
8 & $\mathrm{AO} \mathrm{Cl}(1)$ & 0.2 & 0.1 & 0.2 & 0.1 \\
9 & $\mathrm{AO} \mathrm{Cl}(1)$ & 0.3 & -0.1 & -0.2 & 0.0 \\
10 & $\mathrm{AO} \mathrm{Cl}(1)$ & 0.0 & 0.3 & 0.3 & 0.2 \\
11 & $\mathrm{AO} \mathrm{Cl}(1)$ & 0.0 & -0.6 & -0.4 & -0.3 \\
12 & $\mathrm{AO} \mathrm{Si}(2)$ & 84.6 & 82.7 & 72.9 & 80.1 \\
13 & $\mathrm{AO} \mathrm{Si}(2)$ & 90.8 & 77.5 & 76.4 & 81.5 \\
14 & $\mathrm{AO} \mathrm{Si}(2)$ & 77.3 & 73.8 & 94.4 & 81.8 \\
15 & $\mathrm{AO} \mathrm{Si}(2)$ & 68.8 & 79.5 & 91.3 & 79.9 \\
16 & $\mathrm{BOND} \mathrm{H(10)-C(9)}$ & -0.8 & -0.1 & -2.0 & -1.0 \\
17 & $\mathrm{BONDO}(3)-\mathrm{C}(13)$ & -8.3 & 1.4 & -2.1 & -3.0 \\
18 & $\mathrm{LP} \mathrm{Cl}(1)$ & 8.0 & -0.6 & -6.2 & 0.4
\end{tabular}




\begin{tabular}{lccccc}
19 & BOND H(8)-C(5) & -3.6 & -3.2 & 2.7 & -1.4 \\
20 & BOND H(14)-C(13) & -1.5 & -0.7 & -0.7 & -1.0 \\
21 & LP O(4) & -2.8 & 9.1 & -3.6 & 0.9 \\
22 & LP O(3) & 8.2 & -1.1 & 9.9 & 5.7 \\
23 & BOND O(4)-C(9) & -3.4 & 3.6 & -15.1 & -5.0 \\
24 & BOND H(11)-C(9) & -0.6 & 0.7 & -0.1 & 0.0 \\
25 & BOND H(16)-C(13) & 0.1 & 0.0 & -0.3 & 0.0 \\
26 & BOND H(12)-C(9) & -0.2 & 0.4 & -0.1 & 0.0 \\
27 & BOND H(15)-C(13) & 0.4 & 0.1 & -0.7 & -0.1 \\
28 & LP Cl(1) & 4.1 & -5.4 & -2.4 & -1.2 \\
29 & BOND H(6)-C(5) & -1.7 & -2.5 & 2.0 & -0.8 \\
30 & BOND H(7)-C(5) & -3.0 & -1.7 & 1.6 & -1.0 \\
31 & BOND C(5)-Si(2) & -218.8 & -223.1 & 3.8 & -146.0 \\
32 & BOND O(3)-Si(2) & -127.7 & -78.1 & -131.4 & -112.4 \\
33 & BOND Cl(1)-Si(2) & -18.2 & -147.8 & -143.5 & -103.2 \\
34 & LP Cl(1) & 4.3 & -4.5 & -5.4 & -1.9 \\
35 & BOND O(4)-Si(2) & -122.0 & -20.4 & -149.7 & -97.4 \\
36 & LP O(3) & 11.7 & 15.6 & -0.9 & 8.8 \\
37 & LP O(4) & 7.6 & 6.2 & 2.4 & 5.4 \\
\hline
\end{tabular}

Table 154. MO contributions to $\sigma$ in principal axes for $\mathrm{Si}$ in (MeO) ${ }_{2} \mathrm{MeSiLi}$ [IGLO-BP86/BIII//B3LYP/6$31+G(d)]$.

\begin{tabular}{cccccc} 
MO & type & $\sigma_{1}$ & $\sigma_{2}$ & $\sigma_{3}$ & average \\
\hline 1 & AO Si(2) & 481.9 & 481.9 & 481.9 & 481.9 \\
2 & AO O(4) & 0.0 & 0.0 & 0.0 & 0.0 \\
3 & AO O(3) & 0.0 & 0.0 & 0.0 & 0.0 \\
4 & AO C(13) & 0.0 & 0.0 & 0.0 & 0.0 \\
5 & AO C(9) & 0.0 & 0.0 & 0.0 & 0.0 \\
6 & AO C(5) & 0.0 & 0.0 & 0.0 & 0.0 \\
7 & AO Si(2) & 70.0 & 73.2 & 87.8 & 77.0 \\
8 & AO Si(2) & 90.1 & 87.6 & 63.7 & 80.5 \\
9 & AO Si(2) & 77.9 & 67.9 & 86.4 & 77.4 \\
10 & AO Si(2) & 67.5 & 68.5 & 96.0 & 77.4 \\
11 & AO Li(1) & -0.1 & -0.1 & 0.1 & 0.0 \\
12 & BOND H(11)-C(9) & -0.1 & -0.4 & -0.3 & -0.2 \\
13 & BOND H(10)-C(9) & -0.2 & -0.8 & -1.9 & -1.0 \\
14 & BOND H(16)-C(13) & 0.0 & 1.2 & -0.7 & 0.2 \\
15 & LP O(4) & 2.5 & 12.4 & 7.0 & 7.3 \\
16 & BOND H(15)-C(13) & -3.8 & 1.0 & -0.5 & -1.1 \\
17 & BOND H(6)-C(5) & 1.4 & -0.9 & -2.7 & -0.8 \\
18 & BOND O(3)-Si(2) & -129.7 & -17.8 & -156.2 & -101.2 \\
19 & LP O(3) & 3.8 & 16.1 & 1.6 & 7.1 \\
20 & BOND O(3)-C(13) & -5.0 & 4.3 & -6.2 & -2.3 \\
21 & BOND H(14)-C(13) & -3.7 & -0.2 & -0.7 & -1.5 \\
22 & LP O(4) & 13.7 & -2.1 & 3.0 & 4.9 \\
23 & BOND H(8)-C(5) & -1.0 & -1.5 & -0.6 & -1.0 \\
24 & BOND H(7)-C(5) & -0.3 & -3.0 & -1.1 & -1.5 \\
25 & BOND O(4)-C(9) & 2.8 & 1.0 & -11.5 & -2.6 \\
26 & LP O(3) & 1.0 & 7.0 & 2.6 & 3.5 \\
27 & BOND O(4)-Si(2) & -47.4 & -76.4 & -159.2 & -94.3 \\
28 & BOND C(5)-Si(2) & -120.5 & -207.5 & -124.2 & -150.8 \\
29 & BOND H(12)-C(9) & 0.0 & -0.5 & -0.2 & -0.2 \\
30 & BOND Si(2)-Li(1) & -277.4 & -237.6 & 0.3 & -171.6 \\
\hline & & & & &
\end{tabular}

Table 155. MO contributions to $\sigma$ in principal axes for $\mathrm{Si}$ in (MeO) ${ }_{2} \mathrm{MeSiMe}$ [IGLOBP86/BIII//B3LYP/6-31+G(d)].

\begin{tabular}{llllll} 
MO & type & $\sigma_{1}$ & $\sigma_{2}$ & $\sigma_{3}$ & average \\
\hline
\end{tabular}




\begin{tabular}{|c|c|c|c|c|c|}
\hline 1 & AO Si(2) & 481.9 & 481.9 & 481.9 & 481.9 \\
\hline 2 & $\mathrm{AO} O(4)$ & 0.0 & 0.0 & 0.0 & 0.0 \\
\hline 3 & AO O(5) & 0.0 & 0.0 & 0.0 & 0.0 \\
\hline 4 & $\mathrm{AO} C(6)$ & 0.0 & 0.0 & 0.0 & 0.0 \\
\hline 5 & $\mathrm{AO} C(7)$ & 0.0 & 0.0 & 0.0 & 0.0 \\
\hline 6 & $A O C(1)$ & 0.0 & 0.0 & 0.0 & 0.0 \\
\hline 7 & $\mathrm{AO} C(3)$ & 0.0 & 0.0 & 0.0 & 0.0 \\
\hline 8 & AO Si(2) & 76.2 & 71.7 & 91.6 & 79.8 \\
\hline 9 & AO Si(2) & 91.9 & 84.5 & 64.2 & 80.2 \\
\hline 10 & AO Si(2) & 84.6 & 70.6 & 82.7 & 79.3 \\
\hline 11 & AO Si(2) & 65.2 & 78.0 & 96.1 & 79.8 \\
\hline 12 & BOND H(19)-C(7) & 0.2 & 0.1 & -0.7 & -0.1 \\
\hline 13 & BOND O (4)-C(6) & -2.0 & 2.6 & -10.0 & -3.2 \\
\hline 14 & BOND $\mathrm{H}(10)-C(1)$ & -2.4 & -1.9 & 0.7 & -1.2 \\
\hline 15 & BOND H(18)-C(7) & -1.0 & -0.4 & -1.3 & -0.9 \\
\hline 16 & BOND H(13)-C(3) & 0.7 & -1.7 & -0.9 & -0.6 \\
\hline 17 & BOND H(15)-C(6) & -1.0 & -0.4 & -1.3 & -0.9 \\
\hline 18 & LP O(5) & 5.4 & 12.6 & 3.9 & 7.3 \\
\hline 19 & BOND O(4)-Si(2) & -85.2 & -40.2 & -166.6 & -97.3 \\
\hline 20 & BOND H(17)-C(7) & 0.2 & 0.2 & -0.8 & -0.1 \\
\hline 21 & BOND O(5)-C(7) & -1.5 & 2.4 & -9.9 & -3.0 \\
\hline 22 & BOND H(16)-C(6) & 0.2 & 0.2 & -0.7 & -0.1 \\
\hline 23 & BOND H(14)-C(6) & 0.2 & 0.3 & -0.8 & -0.1 \\
\hline 24 & BOND H(12)-C(3) & -2.9 & -2.8 & 1.4 & -1.4 \\
\hline 25 & BOND H(8)-C(1) & 0.7 & -1.7 & -0.9 & -0.6 \\
\hline 26 & BOND $\mathrm{H}(9)-\mathrm{C}(1)$ & -2.8 & -2.8 & 1.4 & -1.4 \\
\hline 27 & BOND H(11)-C(3) & -2.5 & -1.8 & 0.8 & -1.1 \\
\hline 28 & LP O(4) & 9.6 & 2.0 & 3.8 & 5.1 \\
\hline 29 & BOND C(3)-Si(2) & -164.2 & -200.5 & -58.2 & -141.0 \\
\hline 30 & LP O(4) & 5.4 & 12.6 & 4.0 & 7.4 \\
\hline 31 & BOND C(1)-Si(2) & -165.2 & -200.5 & -58.8 & -141.5 \\
\hline 32 & BOND O(5)-Si(2) & -81.7 & -45.7 & -166.9 & -98.1 \\
\hline 33 & LP O(5) & 10.0 & 1.3 & 4.1 & 5.1 \\
\hline
\end{tabular}

Table 156. MO contributions to $\sigma$ in principal axes for $\mathrm{Si}$ in $\left(\mathrm{Me}_{2} \mathrm{~N}\right)_{2} \mathrm{MeSiH}$ [IGLO-BP86/BIII//B3LYP/6$31+G(d)]$.

\begin{tabular}{cccccc} 
MO & type & $\sigma_{1}$ & $\sigma_{2}$ & $\sigma_{3}$ & average \\
\hline 1 & AO Si(2) & 481.9 & 481.9 & 481.9 & 481.9 \\
2 & AO N(3) & 0.0 & 0.0 & 0.0 & 0.0 \\
3 & AO N(8) & 0.0 & 0.0 & 0.0 & 0.0 \\
4 & AO C(14) & 0.0 & 0.0 & 0.0 & 0.0 \\
5 & AO C(9) & 0.0 & 0.0 & 0.0 & 0.0 \\
6 & AO C(18) & 0.0 & 0.0 & 0.0 & 0.0 \\
7 & AO C(4) & 0.0 & 0.0 & 0.0 & 0.0 \\
8 & AO C(13) & 0.0 & 0.0 & 0.0 & 0.0 \\
9 & AO Si(2) & 85.7 & 73.0 & 75.5 & 78.1 \\
10 & AO Si(2) & 87.7 & 79.3 & 69.1 & 78.7 \\
11 & AO Si(2) & 76.5 & 73.1 & 82.8 & 77.5 \\
12 & AO Si(2) & 70.7 & 74.1 & 84.7 & 76.5 \\
13 & BOND H(15)-C(14) & -0.3 & 0.2 & -0.1 & 0.0 \\
14 & BOND H(10)-C(9) & 0.1 & -0.1 & -0.4 & -0.2 \\
15 & BOND H(19)-C(18) & -0.5 & 0.0 & -0.2 & -0.2 \\
16 & BOND H(5)-C(4) & -0.3 & -0.4 & -0.5 & -0.4 \\
17 & BOND H(23)-C(13) & -1.6 & -1.3 & 0.0 & -1.0 \\
18 & BOND N(3)-C(4) & -4.6 & 1.9 & -3.3 & -2.0 \\
19 & BOND N(8)-Si(2) & -119.3 & -54.0 & -98.8 & -90.7 \\
20 & BOND H(11)-C(9) & -0.5 & -0.6 & -0.1 & -0.4 \\
21 & BOND H(17)-C(14) & -1.3 & 0.4 & 0.2 & -0.2 \\
22 & BOND H(6)-C(4) & 0.2 & -0.7 & -2.4 & -0.9
\end{tabular}




\begin{tabular}{lccccc}
23 & BOND H(7)-C(4) & 1.1 & 0.8 & -0.2 & 0.6 \\
24 & BOND H(16)-C(14) & -0.1 & 0.7 & 0.8 & 0.5 \\
25 & BOND N(8)-C(18) & -5.1 & 2.6 & -3.0 & -1.8 \\
26 & BOND C(13)-Si(2) & -129.3 & -210.1 & -61.3 & -133.6 \\
27 & BOND H(22)-C(13) & -2.3 & -1.7 & 1.6 & -0.8 \\
28 & BOND H(1)-Si(2) & -10.9 & -147.9 & -153.9 & -104.2 \\
29 & BOND H(20)-C(18) & 0.6 & 1.3 & -0.1 & 0.6 \\
30 & BOND H(12)-C(9) & 0.4 & 0.1 & 0.9 & 0.5 \\
31 & BOND H(21)-C(18) & -0.4 & 0.5 & -1.8 & -0.6 \\
32 & BOND N(3)-C(14) & -8.7 & 2.2 & -2.4 & -3.0 \\
33 & LP N(3) & -22.1 & 1.4 & 0.1 & -6.9 \\
34 & BOND N(8)-C(9) & -6.4 & -0.1 & -2.8 & -3.1 \\
35 & BOND H(24)-C(13) & 0.3 & -3.0 & 0.0 & -0.9 \\
36 & BOND N(3)-Si(2) & -140.8 & -37.2 & -93.1 & -90.4 \\
37 & LP N(8) & -25.6 & 1.3 & 4.8 & -6.5 \\
\hline
\end{tabular}

Table 157. MO contributions to $\sigma$ in principal axes for $\mathrm{Si}$ in $\left(\mathrm{Me}_{2} \mathrm{~N}\right)_{2} \mathrm{MeSiCl}$ [IGLO-BP86/BIII//B3LYP/6$31+G(d)]$.

\begin{tabular}{|c|c|c|c|c|c|}
\hline MO & type & $\sigma_{1}$ & $\sigma_{2}$ & $\sigma_{3}$ & average \\
\hline 1 & $\mathrm{AO} \mathrm{Cl}(1)$ & 0.0 & 0.0 & 0.0 & 0.0 \\
\hline 2 & AO Si(2) & 481.9 & 481.9 & 481.9 & 481.9 \\
\hline 3 & AO N(7) & 0.0 & 0.0 & 0.0 & 0.0 \\
\hline 4 & AO N(10) & 0.0 & 0.0 & 0.0 & 0.0 \\
\hline 5 & $\mathrm{AO} C(8)$ & 0.0 & 0.0 & 0.0 & 0.0 \\
\hline 6 & AO C(11) & 0.0 & 0.0 & 0.0 & 0.0 \\
\hline 7 & $\mathrm{AO} C(9)$ & 0.0 & 0.0 & 0.0 & 0.0 \\
\hline 8 & AO C(12) & 0.0 & 0.0 & 0.0 & 0.0 \\
\hline 9 & $\mathrm{AO} C(3)$ & 0.0 & 0.1 & 0.0 & 0.0 \\
\hline 10 & $\mathrm{AO} \mathrm{Cl}(1)$ & 0.1 & -0.4 & -0.3 & -0.2 \\
\hline 11 & $\mathrm{AO} \mathrm{Cl}(1)$ & 0.2 & 0.0 & 0.0 & 0.1 \\
\hline 12 & $\mathrm{AO} \mathrm{Cl}(1)$ & 0.1 & -0.2 & -0.2 & -0.1 \\
\hline 13 & $\mathrm{AO} \mathrm{Cl}(1)$ & 0.1 & 0.3 & 0.3 & 0.2 \\
\hline 14 & AO Si(2) & 79.5 & 80.3 & 77.5 & 79.1 \\
\hline 15 & AO Si(2) & 84.5 & 66.5 & 88.7 & 79.9 \\
\hline 16 & AO Si(2) & 64.2 & 81.4 & 82.8 & 76.1 \\
\hline 17 & AO Si(2) & 82.5 & 91.4 & 65.6 & 79.8 \\
\hline 18 & BOND H(20)-C(12) & 0.9 & 0.4 & 0.3 & 0.5 \\
\hline 19 & BOND $\mathrm{H}(16)-\mathrm{C}(8)$ & 0.5 & 0.5 & 0.6 & 0.5 \\
\hline 20 & LP Cl(1) & 6.8 & -2.9 & -6.3 & -0.8 \\
\hline 21 & BOND N(7)-C(9) & -1.4 & -10.1 & 4.0 & -2.5 \\
\hline 22 & BOND H(24)-C(11) & 0.6 & 0.5 & 1.0 & 0.7 \\
\hline 23 & BOND H(5)-C(3) & -2.6 & 0.3 & -1.2 & -1.1 \\
\hline 24 & BOND N(10)-Si(2) & -36.9 & 0.5 & -4.2 & -13.5 \\
\hline 25 & BOND N(7)-Si(2) & -114.3 & -143.8 & -12.8 & -90.3 \\
\hline 26 & BOND H(17)-C(8) & -0.8 & -0.9 & 0.0 & -0.6 \\
\hline 27 & BOND N(10)-Si(2) & -117.1 & -75.3 & -81.3 & -91.2 \\
\hline 28 & BOND H(19)-C(12) & -0.4 & -0.7 & 0.1 & -0.3 \\
\hline 29 & BOND H(18)-C(8) & -1.0 & 0.3 & 0.5 & -0.1 \\
\hline 30 & BOND H(22)-C(11) & 0.4 & 0.0 & -0.1 & 0.1 \\
\hline 31 & BOND H(14)-C(9) & 0.4 & -0.6 & 0.3 & 0.0 \\
\hline 32 & LP Cl(1) & 6.6 & -6.5 & -0.6 & -0.1 \\
\hline 33 & BOND H(6)-C(3) & -2.2 & 3.0 & -2.9 & -0.7 \\
\hline 34 & BOND H(4)-C(3) & -2.5 & 2.4 & -2.3 & -0.8 \\
\hline 35 & BOND H(13)-C(9) & -0.1 & 1.2 & 0.8 & 0.6 \\
\hline 36 & BOND N(10)-C(12) & -7.3 & -0.1 & -4.9 & -4.1 \\
\hline 37 & BOND H(15)-C(9) & -0.6 & -0.7 & 0.3 & -0.3 \\
\hline 38 & BOND H(23)-C(11) & -0.6 & -0.3 & -0.1 & -0.3 \\
\hline 39 & BOND C(3)-Si(2) & -197.1 & -26.8 & -196.3 & -140.1 \\
\hline 40 & BOND H(21)-C(12) & -0.6 & -0.4 & -0.7 & -0.6 \\
\hline
\end{tabular}




\begin{tabular}{cccccc}
41 & BOND N(7)-C(8) & -2.5 & -8.8 & 2.4 & -3.0 \\
42 & $\mathrm{LP} \mathrm{Cl}(1)$ & 5.3 & -4.8 & -3.0 & -0.8 \\
43 & BOND Cl(1)-Si(2) & 2.2 & -143.2 & -120.5 & -87.2 \\
44 & BOND N(7)-Si(2) & -6.0 & -51.2 & -6.6 & -21.3 \\
45 & BOND N(10)-C(11) & -6.7 & 2.9 & -0.4 & -1.4 \\
\hline
\end{tabular}

Table 158. MO contributions to $\sigma$ in principal axes for $\mathrm{Si}$ in $\left(\mathrm{Me}_{2} \mathrm{~N}\right)_{2} \mathrm{MeSiLi}$ [IGLO-BP86/BIII//B3LYP/6$31+G(d)]$.

\begin{tabular}{|c|c|c|c|c|c|}
\hline MO & type & $\sigma_{1}$ & $\sigma_{2}$ & $\sigma_{3}$ & average \\
\hline 1 & $\mathrm{AO} \mathrm{Si(2)}$ & 481.9 & 481.9 & 481.9 & 481.9 \\
\hline 2 & $A O N(3)$ & 0.0 & 0.0 & 0.0 & 0.0 \\
\hline 3 & $A O N(8)$ & 0.0 & 0.0 & 0.0 & 0.0 \\
\hline 4 & AO C (9) & 0.0 & 0.0 & 0.0 & 0.0 \\
\hline 5 & $\mathrm{AO} C(14)$ & 0.0 & 0.0 & 0.0 & 0.0 \\
\hline 6 & $\mathrm{AO} C(4)$ & 0.0 & 0.0 & 0.0 & 0.0 \\
\hline 7 & AO C(18) & 0.0 & 0.0 & 0.0 & 0.0 \\
\hline 8 & $\mathrm{AO} C(13)$ & 0.0 & 0.0 & 0.0 & 0.0 \\
\hline 9 & AO Si(2) & 74.4 & 75.5 & 76.1 & 75.3 \\
\hline 10 & AO Si(2) & 82.1 & 83.2 & 65.6 & 77.0 \\
\hline 11 & AO Si(2) & 77.6 & 61.9 & 87.5 & 75.7 \\
\hline 12 & AO Si(2) & 64.3 & 76.1 & 85.2 & 75.2 \\
\hline 13 & AO Li(1) & -0.1 & -0.1 & 0.1 & 0.0 \\
\hline 14 & BOND H(10)-C(9) & -2.2 & 0.9 & -0.7 & -0.7 \\
\hline 15 & BOND H(5)-C(4) & -0.2 & 0.1 & -0.2 & -0.1 \\
\hline 16 & BOND N(3)-C(14) & -3.7 & 1.0 & -4.6 & -2.5 \\
\hline 17 & LP N(8) & -6.8 & -2.7 & -11.5 & -7.0 \\
\hline 18 & BOND H(24)-C(13) & 1.9 & -4.0 & -0.8 & -1.0 \\
\hline 19 & OND $\mathrm{H}(11)-\mathrm{C}(9)$ & -3.2 & -0.4 & -0.5 & -1.4 \\
\hline 20 & BOND H(15)-C(1 & -1.6 & 1.0 & -0.2 & -0.3 \\
\hline 21 & BOND H(16)-C(14) & -2.3 & -0.4 & -0.6 & -1.1 \\
\hline 22 & BOND H(12)-C(9) & 0.3 & 0.5 & 0.7 & 0.5 \\
\hline 23 & BOND H(7)-C(4) & -2.4 & -1.3 & 0.4 & -1.1 \\
\hline 24 & BOND H(6)-C(4) & 0.0 & -0.1 & -0.6 & -0.2 \\
\hline 25 & BOND H(21)-C(18) & -0.5 & -0.5 & -0.2 & -0.4 \\
\hline 26 & BOND N(8)-C(9) & -3.0 & -0.9 & -4.4 & -2.8 \\
\hline 27 & BOND H(20)-C(18) & 0.2 & 0.8 & 0.5 & 0.5 \\
\hline 28 & BOND H(23)-C(13) & 1.0 & -0.9 & -2.5 & -0.8 \\
\hline 29 & BOND H(17)-C(14) & -0.4 & 0.5 & -1.0 & -0.3 \\
\hline 30 & BOND H(22)-C(13) & 2.3 & -0.5 & -3.4 & -0.6 \\
\hline 31 & BOND N(8)-C(18) & -3.2 & -1.6 & -4.2 & -3.0 \\
\hline 32 & BOND H(19)-C(18) & 0.1 & -0.6 & 0.1 & -0.1 \\
\hline 33 & BOND N(3)-C(4) & -2.7 & 0.8 & -2.6 & -1.5 \\
\hline 34 & BOND C(13)-Si(2) & -52.1 & -176.2 & -164.1 & -130.8 \\
\hline 35 & BOND N(3)-Si(2) & -119.0 & -51.6 & -144.9 & -105.2 \\
\hline 36 & BOND Si(2)-Li(1) & -258.8 & -185.2 & -10.5 & -151.5 \\
\hline 37 & BOND N(8)-Si(2) & -99.7 & -54.6 & -100.9 & -85.0 \\
\hline 38 & LP N(3) & -3.4 & 7.1 & 2.3 & 2.0 \\
\hline
\end{tabular}

Table 159. MO contributions to $\sigma$ in principal axes for $\mathrm{Si}$ in $\left(\mathrm{Me}_{2} \mathrm{~N}\right)_{2} \mathrm{MeSiMe}$ [IGLOBP86/BIII//B3LYP/6-31+G(d)].

\begin{tabular}{cccccc} 
MO & type & $\sigma_{1}$ & $\sigma_{2}$ & $\sigma_{3}$ & average \\
\hline 1 & AO Si(2) & 481.9 & 481.9 & 481.9 & 481.9 \\
2 & AO N(3) & 0.0 & 0.0 & 0.0 & 0.0 \\
3 & AO N(8) & 0.0 & 0.0 & 0.0 & 0.0 \\
4 & AO C(14) & 0.0 & 0.0 & 0.0 & 0.0 \\
5 & AO C(9) & 0.0 & 0.0 & 0.0 & 0.0 \\
6 & AO C(4) & 0.0 & 0.0 & 0.0 & 0.0
\end{tabular}




\begin{tabular}{cccccc}
7 & AO C(18) & 0.0 & 0.0 & 0.0 & 0.0 \\
8 & AO C(13) & 0.0 & 0.0 & 0.0 & 0.0 \\
9 & AO C(1) & 0.0 & 0.0 & 0.0 & 0.0 \\
10 & AO Si(2) & 87.5 & 75.8 & 70.6 & 78.0 \\
11 & AO Si(2) & 65.8 & 84.7 & 79.6 & 76.7 \\
12 & AO Si(2) & 77.0 & 83.3 & 74.9 & 78.4 \\
13 & AO Si(2) & 85.0 & 64.7 & 80.3 & 76.7 \\
14 & BOND H(20)-C(18) & 0.0 & 1.0 & 0.7 & 0.5 \\
15 & BOND H(16)-C(14) & -0.8 & 0.0 & -0.1 & -0.3 \\
16 & BOND N(8)-C(18) & -5.8 & -1.0 & 1.1 & -1.9 \\
17 & BOND H(26)-C(1) & -1.0 & 0.5 & -1.6 & -0.7 \\
18 & BOND H(7)-C(4) & 0.0 & -0.1 & 0.4 & 0.1 \\
19 & BOND H(22)-C(13) & -0.6 & 0.1 & -1.6 & -0.7 \\
20 & BOND H(11)-C(9) & -0.2 & -1.2 & -0.6 & -0.6 \\
21 & BOND H(17)-C(14) & -0.2 & -1.2 & -0.6 & -0.6 \\
22 & BOND H(15)-C(14) & 1.0 & 0.0 & 0.3 & 0.4 \\
23 & BOND H(21)-C(18) & -1.0 & 0.0 & 0.2 & -0.3 \\
24 & BOND N(8)-Si(2) & -122.8 & -76.1 & -48.1 & -82.3 \\
25 & BOND H(10)-C(9) & -0.9 & 0.0 & 0.0 & -0.3 \\
26 & BOND N(3)-C(14) & -4.6 & -2.9 & -2.0 & -3.1 \\
27 & BOND H(6)-C(4) & -1.0 & 0.0 & 0.2 & -0.2 \\
28 & BOND H(25)-C(1) & -1.1 & -1.0 & -1.0 & -1.1 \\
29 & BOND H(27)-C(1) & 1.7 & -1.7 & -2.4 & -0.8 \\
30 & BOND H(24)-C(13) & -0.8 & -1.4 & -0.9 & -1.0 \\
31 & BOND H(23)-C(13) & 2.1 & -2.1 & -2.5 & -0.8 \\
32 & BOND N(8)-C(9) & -4.1 & -3.3 & -2.1 & -3.2 \\
33 & BOND H(19)-C(18) & 0.0 & -0.1 & 0.4 & 0.1 \\
34 & BOND H(5)-C(4) & 0.0 & 0.9 & 0.7 & 0.5 \\
35 & BOND N(3)-C(4) & -6.4 & -0.6 & 1.1 & -1.9 \\
36 & BOND H(12)-C(9) & 1.0 & 0.0 & 0.3 & 0.4 \\
37 & BOND C(1)-Si(2) & -87.4 & -131.5 & -160.4 & -126.4 \\
38 & BOND N(3)-Si(2) & -20.3 & -11.6 & -2.4 & -11.4 \\
39 & BOND C(13)-Si(2) & -74.2 & -143.3 & -160.8 & -126.1 \\
40 & BOND N(3)-Si(2) & -126.0 & -71.8 & -46.8 & -81.6 \\
41 & BOND N(8)-Si(2) & -18.2 & -13.2 & -1.4 & -11.0 \\
\hline & & & & & \\
\hline & & & &
\end{tabular}

Table 160. MO contributions to $\sigma$ in principal axes for $\mathrm{Si}$ in (MeS) ${ }_{2} \mathrm{MeSiH}$ [IGLO-BP86/BIII//B3LYP/6$31+G(d)]$.

\begin{tabular}{cccccc} 
MO & type & $\sigma_{1}$ & $\sigma_{2}$ & $\sigma_{3}$ & average \\
\hline 1 & AO S(4) & 0.0 & 0.0 & 0.0 & 0.0 \\
2 & AO S(3) & 0.0 & 0.0 & 0.0 & 0.0 \\
3 & AO Si(2) & 481.9 & 481.9 & 481.9 & 481.9 \\
4 & AO C(9) & 0.0 & 0.0 & 0.0 & 0.0 \\
5 & AO C(13) & 0.0 & 0.0 & 0.0 & 0.0 \\
6 & AO C(5) & 0.0 & 0.0 & 0.0 & 0.0 \\
7 & AO S(4) & -0.5 & 0.2 & -0.5 & -0.3 \\
8 & AO S(3) & -0.3 & -0.4 & 0.2 & -0.2 \\
9 & AO S(4) & -0.2 & 0.1 & -0.3 & -0.1 \\
10 & AO S(3) & 0.0 & 0.2 & 0.2 & 0.1 \\
11 & AO S(4) & 0.3 & 0.2 & 0.3 & 0.3 \\
12 & AO S(3) & -0.2 & -0.2 & -0.2 & -0.2 \\
13 & AO S(4) & 0.2 & 0.1 & 0.2 & 0.2 \\
14 & AO S(3) & 0.4 & 0.3 & 0.1 & 0.3 \\
15 & AO Si(2) & 84.4 & 80.1 & 62.6 & 75.7 \\
16 & AO Si(2) & 65.4 & 82.8 & 79.7 & 76.0 \\
17 & AO Si(2) & 79.4 & 68.5 & 81.9 & 76.6 \\
18 & AO Si(2) & 80.3 & 67.6 & 75.1 & 74.4 \\
19 & BOND H(15)-C(13) & -0.3 & -1.2 & 0.7 & -0.3 \\
20 & BOND H(11)-C(9) & -0.1 & 0.6 & -0.5 & 0.0
\end{tabular}




\begin{tabular}{lccccc}
21 & BOND H(7)-C(5) & 2.0 & -0.7 & -1.7 & -0.2 \\
22 & BOND H(14)-C(13) & -1.3 & -0.2 & -0.1 & -0.6 \\
23 & LP S(3) & -10.5 & -2.5 & 7.1 & -2.0 \\
24 & BOND C(9)-S(4) & -4.3 & 4.5 & -1.1 & -0.3 \\
25 & LP S(4) & -6.0 & 8.4 & -7.2 & -1.6 \\
26 & BOND H(10)-C(9) & -2.0 & 0.1 & 0.9 & -0.3 \\
27 & BOND H(12)-C(9) & -0.5 & 0.5 & -0.6 & -0.2 \\
28 & BOND H(16)-C(13) & -0.6 & -0.5 & 0.7 & -0.1 \\
29 & BOND H(8)-C(5) & 1.0 & -2.9 & 1.2 & -0.2 \\
30 & BOND H(6)-C(5) & -2.2 & -1.0 & 1.2 & -0.7 \\
31 & BOND H(1)-Si(2) & -16.6 & -138.2 & -152.5 & -102.4 \\
32 & BOND C(13)-S(3) & -2.8 & -1.9 & 3.5 & -0.4 \\
33 & BOND C(5)-Si(2) & -120.0 & -185.1 & -97.8 & -134.3 \\
34 & LP S(3) & -3.2 & -7.1 & 9.8 & -0.2 \\
35 & BOND S(4)-Si(2) & -207.3 & 4.5 & -134.9 & -112.6 \\
36 & LP S(4) & -2.9 & 8.4 & -5.3 & 0.1 \\
37 & BOND S(3)-Si(2) & -181.2 & -125.4 & -34.8 & -113.8 \\
\hline
\end{tabular}

Table 161. MO contributions to $\sigma$ in principal axes for $\mathrm{Si}$ in (MeS) ${ }_{2} \mathrm{MeSiCl}$ [IGLO-BP86/BIII//B3LYP/6$31+G(d)]$.

\begin{tabular}{|c|c|c|c|c|c|}
\hline MO & type & $\sigma_{1}$ & $\sigma_{2}$ & $\sigma_{3}$ & average \\
\hline 1 & $\mathrm{AO} \mathrm{Cl(1)}$ & 0.0 & 0.0 & 0.0 & 0.0 \\
\hline 2 & AO S(4) & 0.0 & 0.0 & 0.0 & 0.0 \\
\hline 3 & AO S(3) & 0.0 & 0.0 & 0.0 & 0.0 \\
\hline 4 & AO Si(2) & 481.9 & 481.9 & 481.9 & 481.9 \\
\hline 5 & AO C(13) & 0.0 & 0.0 & 0.0 & 0.0 \\
\hline 6 & $A O C(9)$ & 0.0 & 0.0 & 0.0 & 0.0 \\
\hline 7 & AO C (5) & 0.0 & 0.0 & 0.0 & 0.0 \\
\hline 8 & $\mathrm{AO} \mathrm{Cl}(1)$ & 0.0 & 0.3 & 0.2 & 0.2 \\
\hline 9 & AO S(4) & 0.3 & 0.4 & 0.1 & 0.3 \\
\hline 10 & AO S(3) & 0.0 & -0.2 & -0.2 & -0.1 \\
\hline 11 & AO Cl(1) & 0.2 & -0.1 & -0.1 & 0.0 \\
\hline 12 & $\mathrm{AO} \mathrm{Cl}(1)$ & 0.3 & 0.1 & 0.2 & 0.2 \\
\hline 13 & $\mathrm{AO} \mathrm{Cl}(1)$ & -0.3 & -0.2 & -0.6 & -0.3 \\
\hline 14 & AO S(4) & 0.1 & 0.0 & 0.1 & 0.1 \\
\hline 15 & AO S(3) & -0.4 & 0.1 & -0.5 & -0.3 \\
\hline 16 & AO S(4) & -0.4 & -0.2 & 0.3 & -0.1 \\
\hline 17 & AO S(3) & 0.4 & 0.1 & 0.2 & 0.2 \\
\hline 18 & AO S(4) & -0.1 & -0.4 & -0.1 & -0.2 \\
\hline 19 & AO S(3) & 0.0 & 0.3 & 0.3 & 0.2 \\
\hline 20 & AO Si(2) & 85.2 & 81.0 & 64.8 & 77.0 \\
\hline 21 & AO Si(2) & 76.2 & 72.8 & 85.3 & 78.1 \\
\hline 22 & AO Si(2) & 83.3 & 70.7 & 73.2 & 75.7 \\
\hline 23 & AO Si(2) & 64.6 & 81.4 & 84.9 & 77.0 \\
\hline 24 & LP Cl(1) & 6.1 & -0.3 & -0.6 & 1.7 \\
\hline 25 & BOND H(12)-C(9) & -0.1 & -0.5 & 0.8 & 0.1 \\
\hline 26 & BOND H(16)-C(13) & -0.1 & 0.7 & -0.6 & 0.0 \\
\hline 27 & BOND H(7)-C(5) & -0.3 & -2.6 & 2.3 & -0.2 \\
\hline 28 & BOND H(11)-C(9) & -0.6 & -0.7 & 0.8 & -0.2 \\
\hline 29 & BOND C(9)-S(4) & -3.3 & -3.6 & 4.7 & -0.7 \\
\hline 30 & BOND C(13)-S(3) & -0.4 & 3.7 & -4.0 & -0.2 \\
\hline 31 & LP S(3) & -1.5 & 9.1 & -8.4 & -0.3 \\
\hline 32 & BOND H(14)-C(13) & -1.0 & -0.3 & 1.3 & 0.0 \\
\hline 33 & BOND H(15)-C(13) & -0.4 & 0.7 & -0.8 & -0.2 \\
\hline 34 & LP S(4) & -7.2 & -7.2 & 10.2 & -1.4 \\
\hline 35 & BOND $\mathrm{H}(10)-C(9)$ & -1.8 & 0.4 & -0.2 & -0.5 \\
\hline 36 & BOND H(8)-C(5) & 3.0 & -1.0 & -2.3 & -0.1 \\
\hline 37 & BOND H(6)-C(5) & -1.5 & -0.8 & 0.4 & -0.6 \\
\hline
\end{tabular}




\begin{tabular}{cccccc}
38 & BOND Cl(1)-Si(2) & -66.4 & -81.6 & -145.7 & -97.9 \\
39 & BOND C(5)-Si(2) & -116.3 & -199.5 & -124.7 & -146.8 \\
40 & LP Cl(1) & 0.1 & 2.8 & -3.2 & -0.1 \\
41 & BOND S(3)-Si(2) & -157.9 & -46.6 & -177.6 & -127.4 \\
42 & LP Cl(1) & 5.5 & 2.8 & -6.5 & 0.6 \\
43 & BOND S(4)-Si(2) & -206.2 & -163.4 & -28.9 & -132.8 \\
44 & LP S(3) & -5.2 & 5.3 & -8.2 & -2.7 \\
45 & LP S(4) & -6.8 & -4.7 & 10.3 & -0.4 \\
\hline
\end{tabular}

Table 162. MO contributions to $\sigma$ in principal axes for $\mathrm{Si}$ in (MeS) ${ }_{2} \mathrm{MeSiLi}$ [IGLO-BP86/BIII//B3LYP/6$31+G(d)]$.

\begin{tabular}{|c|c|c|c|c|c|}
\hline $\mathrm{MO}$ & type & $\sigma_{1}$ & $\sigma_{2}$ & $\sigma_{3}$ & average \\
\hline 1 & $\mathrm{AOS}(3)$ & 0.0 & 0.0 & 0.0 & 0.0 \\
\hline 2 & AO S(4) & 0.0 & 0.0 & 0.0 & 0.0 \\
\hline 3 & AO Si(2) & 481.9 & 481.9 & 481.9 & 481.9 \\
\hline 4 & $\mathrm{AO} C(13)$ & 0.0 & 0.0 & 0.0 & 0.0 \\
\hline 5 & $\mathrm{AO} C(9)$ & 0.0 & 0.0 & 0.0 & 0.0 \\
\hline 6 & $A O C(5)$ & 0.1 & 0.0 & 0.0 & 0.0 \\
\hline 7 & AO S(3) & -0.5 & -0.6 & 0.1 & -0.3 \\
\hline 8 & AO S(4) & -0.1 & 0.3 & 0.0 & 0.1 \\
\hline 9 & $\mathrm{AO} S(3)$ & 0.1 & 0.1 & 0.0 & 0.0 \\
\hline 10 & AO S(4) & 0.0 & 0.2 & 0.0 & 0.1 \\
\hline 11 & AO S(3) & -0.1 & 0.1 & 0.2 & 0.0 \\
\hline 12 & $\mathrm{AO} S(4)$ & 0.4 & 0.0 & 0.3 & 0.2 \\
\hline 13 & AO S(3) & 0.3 & 0.3 & 0.2 & 0.3 \\
\hline 14 & AO S(4) & -0.4 & -0.1 & -0.6 & -0.4 \\
\hline 15 & $\mathrm{AO} \mathrm{Si(2)}$ & 83.6 & 63.6 & 72.2 & 73.1 \\
\hline 16 & AO Si(2) & 67.6 & 79.3 & 86.7 & 77.9 \\
\hline 17 & AO Si(2) & 75.0 & 79.4 & 70.1 & 74.8 \\
\hline 18 & AO Si(2) & 81.2 & 74.9 & 65.3 & 73.8 \\
\hline 19 & AO Li(1) & 0.0 & 0.0 & 0.0 & 0.0 \\
\hline 20 & BOND H(11)-C(9) & -0.3 & 0.4 & -0.3 & -0.1 \\
\hline 21 & BOND C(13)-S(3) & -3.2 & -1.0 & 3.4 & -0.3 \\
\hline 22 & BOND H(8)-C(5) & 1.6 & -1.6 & -1.0 & -0.3 \\
\hline 23 & BOND H(10)-C(9) & -2.3 & -0.1 & -0.6 & -1.0 \\
\hline 24 & BOND H(14)-C(13) & -3.0 & -0.5 & 0.1 & -1.1 \\
\hline 25 & BOND C(9)-S(4) & -6.5 & 2.7 & 0.7 & -1.1 \\
\hline 26 & BOND H(12)-C(9) & -0.6 & 0.2 & -0.7 & -0.4 \\
\hline 27 & BOND H(16)-C(1 & -0.3 & -0.9 & 0.5 & -0.2 \\
\hline 28 & BOND H(15)-C(13) & -1.5 & -0.7 & 0.6 & -0.5 \\
\hline 29 & BOND C(5)-Si(2) & -33.4 & -196.7 & -169.8 & -133.3 \\
\hline 30 & BOND H(6)-C(5) & 1.6 & -4.1 & -1.8 & -1.4 \\
\hline 31 & BOND H(7)-C(5) & 3.3 & -1.7 & -2.7 & -0.4 \\
\hline 32 & LP S(4) & -6.7 & 4.2 & -3.9 & -2.1 \\
\hline 33 & LP S(3) & -5.4 & -7.7 & 9.3 & -1.2 \\
\hline 34 & LP S(4) & 1.8 & 2.7 & -5.8 & -0.4 \\
\hline 35 & BOND S(3)-Si(2) & -200.4 & -113.8 & -5.4 & -106.5 \\
\hline 36 & LP S(3) & -12.3 & -5.8 & 6.8 & -3.8 \\
\hline 37 & BOND S(4)-Si(2) & -165.8 & -35.0 & -106.0 & -102.3 \\
\hline 38 & BOND Si(2)-Li(1) & -126.6 & -113.9 & -171.4 & -137.3 \\
\hline
\end{tabular}

Table 163. MO contributions to $\sigma$ in principal axes for $\mathrm{Si}$ in (MeS) ${ }_{2} \mathrm{MeSiMe}$ [IGLOBP86/BIII//B3LYP/6-31+G(d)].

\begin{tabular}{cccccc} 
MO & type & $\sigma_{1}$ & $\sigma_{2}$ & $\sigma_{3}$ & average \\
\hline 1 & AO S(3) & 0.0 & 0.0 & 0.0 & 0.0 \\
2 & AO S(4) & 0.0 & 0.0 & 0.0 & 0.0 \\
3 & AO Si(2) & 481.9 & 481.9 & 481.9 & 481.9
\end{tabular}




\begin{tabular}{|c|c|c|c|c|c|}
\hline 4 & $\mathrm{AO} C(13)$ & 0.0 & 0.0 & 0.0 & 0.0 \\
\hline 5 & $\mathrm{AO} C(9)$ & 0.0 & 0.0 & 0.0 & 0.0 \\
\hline 6 & $\mathrm{AO} C(1)$ & 0.0 & 0.0 & 0.0 & 0.0 \\
\hline 7 & AO C (5) & 0.0 & 0.0 & 0.0 & 0.0 \\
\hline 8 & AO S(3) & -0.1 & 0.3 & -0.3 & 0.0 \\
\hline 9 & AO S(4) & -0.6 & -0.3 & -0.3 & -0.4 \\
\hline 10 & AO S(3) & -0.5 & -0.3 & -0.3 & -0.4 \\
\hline 11 & AO S (4) & 0.0 & 0.3 & -0.2 & 0.1 \\
\hline 12 & AO S(4) & 0.3 & 0.0 & 0.2 & 0.2 \\
\hline 13 & AO S(3) & 0.4 & 0.1 & 0.2 & 0.2 \\
\hline 14 & AO S(3) & 0.1 & 0.1 & 0.3 & 0.2 \\
\hline 15 & AO S(4) & 0.1 & 0.2 & 0.4 & 0.2 \\
\hline 16 & AO Si(2) & 84.1 & 69.4 & 72.3 & 75.3 \\
\hline 17 & AO Si(2) & 65.3 & 79.7 & 85.4 & 76.8 \\
\hline 18 & AO Si(2) & 73.9 & 73.7 & 80.9 & 76.2 \\
\hline 19 & AO Si(2) & 86.7 & 74.4 & 64.0 & 75.0 \\
\hline 20 & BOND C(9)-S(4) & -3.4 & 1.2 & 0.4 & -0.6 \\
\hline 21 & LP S(3) & -7.6 & 3.3 & -1.1 & -1.8 \\
\hline 22 & BOND H(7)-C(5) & 3.1 & -2.0 & -1.9 & -0.2 \\
\hline 23 & BOND H(17)-C(1) & 1.4 & -0.4 & -1.9 & -0.3 \\
\hline 24 & BOND H(12)-C(9) & -0.8 & -0.1 & 0.0 & -0.3 \\
\hline 25 & BOND H(16)-C(13) & -0.8 & 0.0 & 0.0 & -0.3 \\
\hline 26 & BOND H(10)-C(9) & -1.8 & 0.3 & 0.3 & -0.4 \\
\hline 27 & LP S(4) & -7.9 & 3.2 & -0.7 & -1.8 \\
\hline 28 & BOND H(11)-C(9) & -0.2 & 0.1 & 0.0 & 0.0 \\
\hline 29 & BOND H(14)-C(13) & -1.8 & 0.3 & 0.3 & -0.4 \\
\hline 30 & BOND H(15)-C(13) & -0.2 & 0.1 & 0.0 & 0.0 \\
\hline 31 & BOND C(5)-Si(2) & -75.6 & -171.0 & -148.0 & -131.5 \\
\hline 32 & BOND H(8)-C(5) & 1.3 & -0.4 & -1.8 & -0.3 \\
\hline 33 & BOND H(18)-C(1) & -0.6 & -2.0 & 0.3 & -0.8 \\
\hline 34 & BOND $\mathrm{H}(19)-\mathrm{C}(1)$ & 3.1 & -2.0 & -2.0 & -0.3 \\
\hline 35 & BOND H(6)-C(5) & -0.6 & -2.0 & 0.2 & -0.8 \\
\hline 36 & BOND C(13)-S(3) & -3.4 & 1.3 & 0.4 & -0.6 \\
\hline 37 & BOND C(1)-Si(2) & -75.7 & -171.7 & -145.8 & -131.1 \\
\hline 38 & LP S(3) & -4.4 & -1.9 & 3.5 & -0.9 \\
\hline 39 & BOND S(4)-Si(2) & -185.4 & -57.7 & -82.9 & -108.7 \\
\hline 40 & LP S(4) & -4.5 & -2.0 & 3.7 & -0.9 \\
\hline 41 & BOND S(3)-Si(2) & -185.7 & -57.1 & -83.8 & -108.9 \\
\hline
\end{tabular}

Table 164. MO contributions to $\sigma$ in principal axes for $\mathrm{Si}$ in $(\mathrm{MeO})_{3} \mathrm{SiCl}$ [IGLO-BP86/BIII//B3LYP/6$31+G(d)]$.

\begin{tabular}{cccccc} 
MO & type & $\sigma_{1}$ & $\sigma_{2}$ & $\sigma_{3}$ & average \\
\hline 1 & $\mathrm{AO} \mathrm{Cl}(1)$ & 0.0 & 0.0 & 0.0 & 0.0 \\
2 & $\mathrm{AO} \mathrm{Si}(2)$ & 481.9 & 481.9 & 481.9 & 481.9 \\
3 & $\mathrm{AO} \mathrm{O}(13)$ & 0.0 & 0.0 & 0.0 & 0.0 \\
4 & $\mathrm{AO} \mathrm{O}(3)$ & 0.0 & 0.0 & 0.0 & 0.0 \\
5 & $\mathrm{AO} \mathrm{O}(8)$ & 0.0 & 0.0 & 0.0 & 0.0 \\
6 & $\mathrm{AO} \mathrm{C}(4)$ & 0.0 & 0.0 & 0.0 & 0.0 \\
7 & $\mathrm{AO} \mathrm{C}(9)$ & 0.0 & 0.0 & 0.0 & 0.0 \\
8 & $\mathrm{AO} \mathrm{C}(14)$ & 0.0 & 0.0 & 0.0 & 0.0 \\
9 & $\mathrm{AO} \mathrm{Cl}(1)$ & 0.2 & 0.2 & 0.1 & 0.2 \\
10 & $\mathrm{AO} \mathrm{Cl}(1)$ & 0.0 & 0.0 & 0.0 & 0.0 \\
11 & $\mathrm{AO} \mathrm{Cl}(1)$ & 0.1 & 0.2 & 0.2 & 0.2 \\
12 & $\mathrm{AO} \mathrm{Cl}(1)$ & 0.1 & -0.6 & -0.6 & -0.4 \\
13 & $\mathrm{AO} \mathrm{Si}(2)$ & 71.0 & 83.9 & 90.4 & 81.7 \\
14 & $\mathrm{AO} \mathrm{Si}(2)$ & 90.1 & 93.1 & 66.4 & 83.2 \\
15 & $\mathrm{AO} \mathrm{Si}(2)$ & 93.3 & 69.7 & 90.3 & 84.4 \\
16 & $\mathrm{AO} \mathrm{Si}(2)$ & 79.1 & 82.7 & 84.7 & 82.2 \\
17 & $\mathrm{LP} \mathrm{O}(3)$ & 7.1 & 11.0 & 0.1 & 6.1
\end{tabular}




\begin{tabular}{lccccc}
18 & BOND H(10)-C(9) & -1.2 & -1.1 & -0.2 & -0.8 \\
19 & BOND H(16)-C(14) & -1.2 & -0.9 & -0.4 & -0.8 \\
20 & LP Cl(1) & 6.9 & -5.7 & -4.3 & -1.0 \\
21 & BOND H(5)-C(4) & -0.6 & -0.1 & -2.2 & -1.0 \\
22 & BOND O(13)-C(14) & -6.9 & -4.7 & -4.0 & -5.2 \\
23 & LP O(8) & 2.2 & 0.1 & 14.5 & 5.6 \\
24 & BOND H(6)-C(4) & -0.3 & 1.1 & -0.7 & 0.0 \\
25 & BOND H(17)-C(14) & -0.2 & -0.8 & 1.1 & 0.0 \\
26 & BOND H(11)-C(9) & -0.3 & 0.1 & 0.4 & 0.1 \\
27 & BOND H(12)-C(9) & -0.2 & -0.1 & 0.2 & 0.0 \\
28 & BOND O(13)-Si(2) & -4.5 & -7.5 & 6.1 & -2.0 \\
29 & BOND H(7)-C(4) & -0.7 & 1.1 & -0.3 & 0.1 \\
30 & BOND O(8)-Si(2) & -168.2 & -124.6 & -44.8 & -112.5 \\
31 & BOND H(15)-C(14) & -0.6 & -0.4 & 1.0 & 0.0 \\
32 & BOND O(3)-C(4) & -4.0 & 0.0 & -12.6 & -5.5 \\
33 & LP Cl(1) & 5.4 & -5.2 & -2.9 & -0.9 \\
34 & LP O(8) & 5.3 & 7.4 & 4.0 & 5.6 \\
35 & LP Cl(1) & 3.4 & -3.3 & -6.5 & -2.1 \\
36 & BOND O(3)-Si(2) & -119.5 & -39.7 & -150.7 & -103.3 \\
37 & LP O(13) & 4.3 & -4.0 & 11.3 & 3.9 \\
38 & BOND Cl(1)-Si(2) & -7.7 & -149.5 & -150.3 & -102.5 \\
39 & BOND O(8)-C(9) & -11.1 & -2.5 & 3.6 & -3.3 \\
40 & BOND O(13)-Si(2) & -133.5 & -100.3 & -58.0 & -97.3 \\
41 & LP O(3) & 0.9 & 14.6 & -3.2 & 4.1 \\
\hline
\end{tabular}

Table 165. MO contributions to $\sigma$ in principal axes for Si in (MeO) ${ }_{3}$ SiLi [IGLO-BP86/BIII//B3LYP/6$31+G(d)]$.

\begin{tabular}{cccccc} 
MO & type & $\sigma_{1}$ & $\sigma_{2}$ & $\sigma_{3}$ & average \\
\hline 1 & AO Si(2) & 481.9 & 481.9 & 481.9 & 481.9 \\
2 & AO O(13) & 0.0 & 0.0 & 0.0 & 0.0 \\
3 & AO O(8) & 0.0 & 0.0 & 0.0 & 0.0 \\
4 & AO O(3) & 0.0 & 0.0 & 0.0 & 0.0 \\
5 & AO C(14) & 0.0 & 0.0 & 0.0 & 0.0 \\
6 & AO C(9) & 0.0 & 0.0 & 0.0 & 0.0 \\
7 & AO C(4) & 0.0 & 0.0 & 0.0 & 0.0 \\
8 & AO Si(2) & 78.6 & 70.0 & 91.1 & 79.9 \\
9 & AO Si(2) & 92.7 & 92.4 & 62.4 & 82.5 \\
10 & AO Si(2) & 64.9 & 81.9 & 93.5 & 80.1 \\
11 & AO Si(2) & 76.8 & 68.8 & 92.9 & 79.5 \\
12 & AO Li(1) & -0.1 & -0.1 & 0.2 & 0.0 \\
13 & BOND H(16)-C(14) & -4.0 & 0.7 & -0.1 & -1.1 \\
14 & BOND H(10)-C(9) & -1.7 & -0.8 & -0.9 & -1.1 \\
15 & BOND O(3)-C(4) & 2.5 & -2.5 & -12.1 & -4.0 \\
16 & BOND O(13)-C(14) & -4.8 & -1.8 & -4.7 & -3.8 \\
17 & BOND O(8)-C(9) & -7.0 & 0.1 & -5.7 & -4.2 \\
18 & BOND H(6)-C(4) & -0.7 & -0.3 & 0.5 & -0.2 \\
19 & BOND H(11)-C(9) & 0.0 & -1.7 & -0.1 & -0.6 \\
20 & BOND H(12)-C(9) & 0.7 & 0.4 & -0.4 & 0.2 \\
21 & LP O(3) & 4.2 & -9.3 & -5.2 & -3.4 \\
22 & BOND H(17)-C(14) & -2.4 & -0.6 & -0.7 & -1.2 \\
23 & BOND H(15)-C(14) & -0.2 & 1.2 & -0.5 & 0.2 \\
24 & BOND H(7)-C(4) & -0.4 & -0.4 & 0.2 & -0.2 \\
25 & BOND H(5)-C(4) & 0.0 & -0.5 & -1.6 & -0.7 \\
26 & LP O(13) & -3.2 & 11.6 & 3.8 & 4.1 \\
27 & LP O(8) & 11.8 & 7.3 & -0.9 & 6.1 \\
28 & BOND O(8)-Si(2) & -119.6 & -63.9 & -131.0 & -104.8 \\
29 & BOND O(13)-Si(2) & -121.7 & -66.1 & -134.7 & -107.5 \\
30 & LP O(13) & 2.2 & 16.4 & -0.6 & 6.0 \\
31 & BOND O(3)-Si(2) & -22.8 & -123.0 & -126.5 & -90.8
\end{tabular}




\begin{tabular}{lccccc}
32 & LP O(8) & 7.7 & -0.9 & 3.9 & 3.6 \\
33 & LP O(3) & 7.1 & -1.9 & 0.4 & 1.9 \\
34 & BOND Si(2)-Li(1) & -248.9 & -245.5 & 11.1 & -161.1 \\
\hline
\end{tabular}

Table 166. MO contributions to $\sigma$ in principal axes for $\mathrm{Si}$ in $\left(\mathrm{Me}_{2} \mathrm{~N}\right)_{3} \mathrm{SiCl}$ [IGLO-BP86/BIII//B3LYP/6$31+G(d)]$.

\begin{tabular}{|c|c|c|c|c|c|}
\hline $\mathrm{MO}$ & type & $\sigma_{1}$ & $\sigma_{2}$ & $\sigma_{3}$ & average \\
\hline 1 & $\mathrm{AO} \mathrm{Cl}(1)$ & 0.0 & 0.0 & 0.0 & 0.0 \\
\hline 2 & AO Si(2) & 481.9 & 481.9 & 481.9 & 481.9 \\
\hline 3 & AO N(13) & 0.0 & 0.1 & 0.0 & 0.0 \\
\hline 4 & AO N(8) & 0.0 & 0.0 & 0.0 & 0.0 \\
\hline 5 & $\mathrm{AO} N(3)$ & 0.0 & 0.0 & 0.1 & 0.0 \\
\hline 6 & AO C(14) & 0.0 & 0.0 & 0.0 & 0.0 \\
\hline 7 & $\mathrm{AO} C(9)$ & 0.0 & 0.0 & 0.0 & 0.0 \\
\hline 8 & $\mathrm{AO} C(26)$ & 0.0 & 0.0 & 0.0 & 0.0 \\
\hline 9 & $\mathrm{AO} C(22)$ & 0.0 & 0.0 & 0.0 & 0.0 \\
\hline 10 & AO C(18) & 0.0 & 0.0 & 0.0 & 0.0 \\
\hline 11 & $\mathrm{AO} C(4)$ & 0.0 & 0.0 & 0.0 & 0.0 \\
\hline 12 & $\mathrm{AO} \mathrm{Cl}(1)$ & 0.2 & -0.3 & -0.3 & -0.1 \\
\hline 13 & $\mathrm{AO} \mathrm{Cl}(1)$ & 0.1 & 0.1 & 0.1 & 0.1 \\
\hline 14 & $\mathrm{AO} \mathrm{Cl}(1)$ & 0.1 & -0.3 & -0.4 & -0.2 \\
\hline 15 & $\mathrm{AO} \mathrm{Cl}(1)$ & 0.1 & 0.3 & 0.3 & 0.2 \\
\hline 16 & AO Si(2) & 78.4 & 89.7 & 68.5 & 78.9 \\
\hline 17 & AO Si(2) & 86.8 & 71.3 & 83.9 & 80.6 \\
\hline 18 & AO Si(2) & 64.4 & 82.4 & 82.6 & 76.5 \\
\hline 19 & AO Si(2) & 83.5 & 72.9 & 83.0 & 79.8 \\
\hline 20 & BOND H(27)-C(26) & -0.3 & 1.2 & 1.3 & 0.7 \\
\hline 21 & BOND H(28)-C(26) & 1.0 & 0.1 & -0.6 & 0.2 \\
\hline 22 & BOND H(20)-C(18) & 1.2 & -1.4 & 0.5 & 0.1 \\
\hline 23 & $\mathrm{LP} \mathrm{Cl}(1)$ & 6.0 & -5.2 & -5.2 & -1.5 \\
\hline 24 & BOND N(8)-C(9) & -5.3 & -0.4 & -4.0 & -3.2 \\
\hline 25 & BOND H(17)-C(14) & -1.0 & 0.9 & 1.6 & 0.5 \\
\hline 26 & BOND H(7)-C(4) & -0.4 & 1.4 & 0.4 & 0.5 \\
\hline 27 & BOND H(23)-C(22) & -0.3 & 0.2 & 1.2 & 0.4 \\
\hline 28 & BOND H(25)-C(22) & -0.2 & 0.0 & -0.9 & -0.4 \\
\hline 29 & BOND H(12)-C(9) & -1.5 & 0.4 & 1.2 & 0.0 \\
\hline 30 & BOND H(19)-C(18) & -1.0 & 1.4 & 0.7 & 0.4 \\
\hline 31 & BOND H(16)-C(14) & -1.6 & 0.2 & -0.8 & -0.7 \\
\hline 32 & BOND N(3)-Si(2) & -102.7 & -66.4 & 3.0 & -55.4 \\
\hline 33 & BOND H(11)-C(9) & -1.3 & -0.4 & -0.7 & -0.8 \\
\hline 34 & BOND N(13)-C(14) & -7.4 & 4.0 & -4.7 & -2.7 \\
\hline 35 & BOND N(8)-C(26) & -2.9 & -1.3 & -3.9 & -2.7 \\
\hline 36 & BOND H(6)-C(4) & -0.3 & -0.9 & 0.4 & -0.3 \\
\hline 37 & BOND N(13)-C(22) & -6.0 & 3.1 & -7.9 & -3.6 \\
\hline 38 & BOND H(29)-C(26) & -0.2 & 0.1 & -0.4 & -0.2 \\
\hline 39 & $\mathrm{LP} \mathrm{Cl}(1)$ & 4.8 & -3.9 & -4.9 & -1.3 \\
\hline 40 & BOND H(15)-C(14) & 1.0 & -0.2 & -0.7 & 0.1 \\
\hline 41 & BOND H(10)-C(9) & 0.8 & 0.8 & -0.5 & 0.4 \\
\hline 42 & BOND H(5)-C(4) & 1.3 & -0.6 & 0.7 & 0.5 \\
\hline 43 & BOND H(24)-C(22) & 1.2 & 0.4 & -0.3 & 0.4 \\
\hline 44 & BOND H(21)-C(18) & -1.0 & -1.8 & 0.1 & -0.9 \\
\hline 45 & BOND N(3)-C(4) & -5.0 & -8.6 & 4.4 & -3.1 \\
\hline 46 & BOND N(13)-Si(2) & -68.9 & -33.5 & -87.1 & -63.2 \\
\hline 47 & BOND N(8)-Si(2) & -122.9 & -52.6 & -74.4 & -83.3 \\
\hline 48 & BOND N(3)-C(18) & -6.9 & -7.2 & 4.2 & -3.3 \\
\hline 49 & BOND Cl(1)-Si(2) & 3.6 & -130.6 & -126.9 & -84.6 \\
\hline 50 & LP Cl(1) & 4.7 & -4.9 & -4.0 & -1.4 \\
\hline 51 & BOND N(13)-Si(2) & -87.9 & 1.4 & -50.3 & -45.6 \\
\hline 52 & BOND N(3)-Si(2) & -48.8 & -107.6 & -6.6 & -54.3 \\
\hline
\end{tabular}


Table 167. $\mathrm{MO}$ contributions to $\sigma$ in principal axes for $\mathrm{Si}$ in $\left(\mathrm{Me}_{2} \mathrm{~N}\right)_{3} \mathrm{SiLi}$ [IGLO-BP86/BIII//B3LYP/6$31+G(d)]$.

\begin{tabular}{|c|c|c|c|c|c|}
\hline $\mathrm{MO}$ & type & $\sigma_{1}$ & $\sigma_{2}$ & $\sigma_{3}$ & average \\
\hline 1 & AO Si(2) & 481.9 & 481.9 & 481.9 & 481.9 \\
\hline 2 & AO N(8) & 0.0 & 0.0 & 0.0 & 0.0 \\
\hline 3 & $\mathrm{AO} N(3)$ & 0.0 & 0.0 & 0.0 & 0.0 \\
\hline 4 & AO N(13) & 0.0 & 0.1 & 0.0 & 0.0 \\
\hline 5 & $\mathrm{AO} C(9)$ & 0.0 & 0.0 & 0.0 & 0.0 \\
\hline 6 & AO C(4) & 0.0 & 0.0 & 0.0 & 0.0 \\
\hline 7 & $\mathrm{AO} C(26)$ & 0.0 & 0.0 & 0.0 & 0.0 \\
\hline 8 & AO C(18) & 0.0 & 0.0 & 0.0 & 0.0 \\
\hline 9 & AO C(14) & 0.0 & 0.0 & 0.0 & 0.0 \\
\hline 10 & AO $C(22)$ & 0.0 & 0.0 & 0.0 & 0.0 \\
\hline 11 & AO Si(2) & 78.0 & 75.9 & 76.1 & 76.7 \\
\hline 12 & AO Si(2) & 82.9 & 80.0 & 69.5 & 77.5 \\
\hline 13 & AO Si(2) & 70.4 & 69.1 & 91.4 & 77.0 \\
\hline 14 & AO Si(2) & 65.9 & 76.5 & 84.6 & 75.7 \\
\hline 15 & AO Li(1) & -0.1 & -0.1 & 0.2 & 0.0 \\
\hline 16 & BOND N(8)-C(9) & 2.1 & -5.0 & -4.2 & -2.3 \\
\hline 17 & BOND H(7)-C(4) & 1.4 & -0.6 & 0.7 & 0.5 \\
\hline 18 & BOND N(3)-C(4) & 2.1 & -4.6 & -4.1 & -2.2 \\
\hline 19 & BOND N(3)-C(18) & 0.4 & -5.3 & -6.2 & -3.7 \\
\hline 20 & BOND H(28)-C(26) & -0.1 & -0.4 & -0.5 & -0.3 \\
\hline 21 & BOND H(24)-C(22) & -3.4 & 0.5 & -0.8 & -1.2 \\
\hline 22 & BOND H(12)-C(9) & -0.6 & 0.0 & -0.5 & -0.4 \\
\hline 23 & BOND H(16)-C(14) & -0.5 & 0.5 & -1.1 & -0.4 \\
\hline 24 & BOND H(17)-C(14) & -3.4 & 0.5 & -0.6 & -1.2 \\
\hline 25 & BOND H(6)-C(4) & -0.1 & -2.7 & -0.3 & -1.0 \\
\hline 26 & BOND H(21)-C(18) & -0.2 & -0.2 & -0.6 & -0.3 \\
\hline 27 & BOND H(5)-C(4) & -0.6 & 0.1 & -0.6 & -0.4 \\
\hline 28 & BOND N(8)-C(26) & 0.7 & -5.6 & -5.9 & -3.6 \\
\hline 29 & BOND H(27)-C(26) & 0.0 & 0.6 & 0.2 & 0.3 \\
\hline 30 & BOND N(13)-C(14) & -6.8 & 3.1 & -3.6 & -2.4 \\
\hline 31 & BOND H(10)-C(9) & 1.6 & -0.8 & 0.7 & 0.5 \\
\hline 32 & BOND N(13)-C(22) & -6.8 & 3.1 & -3.9 & -2.5 \\
\hline 33 & BOND H(19)-C(18) & -0.2 & -0.5 & -0.4 & -0.4 \\
\hline 34 & LP N(3) & 4.3 & -9.0 & -22.1 & -8.9 \\
\hline 35 & BOND H(29)-C(26) & -0.2 & -0.2 & -0.5 & -0.3 \\
\hline 36 & BOND H(23)-C(22) & -1.1 & 0.6 & -0.1 & -0.2 \\
\hline 37 & BOND H(15)-C(14) & -0.9 & 0.6 & -0.1 & -0.2 \\
\hline 38 & BOND N(3)-Si(2) & -44.7 & -111.7 & -110.5 & -89.0 \\
\hline 39 & LP N(8) & 5.3 & -13.1 & -21.8 & -9.9 \\
\hline 40 & BOND H(25)-C(22) & -0.6 & 0.5 & -1.2 & -0.4 \\
\hline 41 & BOND H(11)-C(9) & 0.1 & -3.1 & -0.4 & -1.1 \\
\hline 42 & BOND N(13)-Si(2) & -178.5 & -2.4 & -140.8 & -107.3 \\
\hline 43 & BOND Si(2)-Li(1) & -226.8 & -227.5 & 0.9 & -151.1 \\
\hline 44 & BOND H(20)-C(18) & 0.1 & 0.7 & 0.1 & 0.3 \\
\hline 45 & BOND N(8)-Si(2) & -41.5 & -112.8 & -109.9 & -88.0 \\
\hline 46 & LP N(13) & -7.8 & 4.4 & 0.2 & -1.1 \\
\hline
\end{tabular}

Table 168. MO contributions to $\sigma$ in principal axes for $\mathrm{Si}$ in (MeS) $)_{3} \mathrm{SiCl}$ [IGLO-BP86/BIII//B3LYP/6$31+G(d)]$.

\begin{tabular}{cccccc} 
MO & type & $\sigma_{1}$ & $\sigma_{2}$ & $\sigma_{3}$ & average \\
\hline 1 & $\mathrm{AO} \mathrm{Cl}(1)$ & 0.0 & 0.0 & 0.0 & 0.0 \\
2 & $\mathrm{AO} \mathrm{S}(3)$ & 0.0 & 0.0 & 0.0 & 0.0
\end{tabular}




\begin{tabular}{|c|c|c|c|c|c|}
\hline 3 & AO S(13) & 0.0 & 0.0 & 0.0 & 0.0 \\
\hline 4 & AO S(8) & 0.0 & 0.0 & 0.0 & 0.0 \\
\hline 5 & AO Si(2) & 481.9 & 481.9 & 481.9 & 481.9 \\
\hline 6 & AO C(14) & 0.0 & 0.0 & 0.0 & 0.0 \\
\hline 7 & $\mathrm{AO} C(4)$ & 0.0 & 0.0 & 0.0 & 0.0 \\
\hline 8 & AO C (9) & 0.0 & 0.0 & 0.0 & 0.0 \\
\hline 9 & $\mathrm{AO} \mathrm{Cl}(1)$ & -0.1 & -0.3 & -0.1 & -0.1 \\
\hline 10 & AO S(3) & 0.0 & 0.0 & -0.3 & -0.1 \\
\hline 11 & AO S(13) & 0.2 & 0.3 & 0.3 & 0.3 \\
\hline 12 & AO S(8) & -0.2 & 0.3 & 0.1 & 0.1 \\
\hline 13 & $\mathrm{AO} \mathrm{Cl(1)}$ & -0.1 & 0.2 & 0.1 & 0.1 \\
\hline 14 & $\mathrm{AO} \mathrm{Cl}(1)$ & 0.1 & -0.2 & -0.4 & -0.2 \\
\hline 15 & $\mathrm{AO} \mathrm{Cl}(1)$ & 0.3 & 0.2 & 0.3 & 0.2 \\
\hline 16 & AO S(3) & 0.1 & -0.2 & 0.3 & 0.1 \\
\hline 17 & AO S(13) & 0.3 & 0.0 & -0.2 & 0.0 \\
\hline 18 & AO S(8) & 0.1 & -0.1 & 0.2 & 0.1 \\
\hline 19 & AO S(3) & -0.5 & -0.1 & -0.2 & -0.2 \\
\hline 20 & AO S(13) & -0.2 & -0.5 & 0.0 & -0.3 \\
\hline 21 & AO S(8) & 0.4 & 0.2 & 0.1 & 0.2 \\
\hline 22 & AO S(13) & -0.2 & 0.0 & 0.1 & 0.0 \\
\hline 23 & AO S(3) & 0.3 & 0.3 & 0.2 & 0.3 \\
\hline 24 & AO S(8) & -0.5 & -0.1 & -0.6 & -0.4 \\
\hline 25 & AO Si(2) & 64.5 & 87.5 & 81.1 & 77.7 \\
\hline 26 & AO Si(2) & 84.0 & 77.6 & 71.4 & 77.7 \\
\hline 27 & AO Si(2) & 76.7 & 78.5 & 71.7 & 75.6 \\
\hline 28 & AO Si(2) & 83.7 & 67.1 & 84.1 & 78.3 \\
\hline 29 & LP Cl(1) & 6.5 & 0.0 & -5.2 & 0.5 \\
\hline 30 & BOND H(10)-C(9) & -1.2 & 0.1 & 0.6 & -0.2 \\
\hline 31 & BOND H(16)-C(14) & 0.2 & -1.4 & 0.4 & -0.3 \\
\hline 32 & LP S(3) & -4.4 & -4.7 & 5.4 & -1.2 \\
\hline 33 & BOND H(7)-C(4) & -0.5 & 0.0 & 0.1 & -0.2 \\
\hline 34 & BOND C(14)-S(13) & 1.1 & -3.2 & 1.6 & -0.2 \\
\hline 35 & BOND H(17)-C(14) & 0.1 & -0.3 & 0.1 & 0.0 \\
\hline 36 & LP S $(8)$ & -1.1 & 10.1 & -6.1 & 0.9 \\
\hline 37 & LP S(3) & -7.4 & 6.9 & -1.0 & -0.5 \\
\hline 38 & LP Cl(1) & 1.9 & 1.2 & -0.1 & 1.0 \\
\hline 39 & BOND C(9)-S(8) & -1.7 & 2.6 & -1.2 & -0.1 \\
\hline 40 & BOND H(12)-C(9) & -0.6 & 0.3 & -0.2 & -0.2 \\
\hline 41 & BOND H(11)-C(9) & 0.1 & 0.5 & -0.4 & 0.1 \\
\hline 42 & BOND H(5)-C(4) & -1.2 & 0.5 & -0.2 & -0.3 \\
\hline 43 & BOND H(15)-C(14) & 0.0 & -0.2 & -0.1 & -0.1 \\
\hline 44 & BOND S(13)-Si(2) & -97.3 & -229.7 & -79.5 & -135.5 \\
\hline 45 & BOND S(3)-Si(2) & -201.3 & -89.9 & -113.2 & -134.8 \\
\hline 46 & BOND H(6)-C(4) & 0.0 & 0.2 & 0.1 & 0.1 \\
\hline 47 & LP Cl(1) & 7.1 & -5.4 & 2.2 & 1.3 \\
\hline 48 & BOND S(8)-Si(2) & -193.6 & -57.0 & -152.4 & -134.3 \\
\hline 49 & LP S(13) & -5.0 & -5.1 & 7.6 & -0.8 \\
\hline 50 & BOND Cl(1)-Si(2) & -53.7 & -133.1 & -124.5 & -103.8 \\
\hline 51 & LP S(8) & -3.7 & -0.3 & 0.5 & -1.2 \\
\hline 52 & LP S(13) & 6.1 & -7.2 & -0.7 & -0.6 \\
\hline 53 & BOND C (4)-S(3) & -1.8 & 1.9 & -0.7 & -0.2 \\
\hline
\end{tabular}

Table 169. MO contributions to $\sigma$ in principal axes for $\mathrm{Si}$ in (MeS) ${ }_{3} \mathrm{SiLi}$ [IGLO-BP86/BIII//B3LYP/6$31+G(d)]$.

\begin{tabular}{cccccc} 
MO & type & $\sigma_{1}$ & $\sigma_{2}$ & $\sigma_{3}$ & average \\
\hline 1 & AO S(13) & 0.0 & 0.0 & 0.0 & 0.0 \\
2 & AO S(8) & 0.0 & 0.0 & 0.0 & 0.0 \\
3 & AO S(3) & 0.0 & 0.0 & 0.0 & 0.0 \\
4 & AO Si(2) & 481.9 & 481.9 & 481.9 & 481.9
\end{tabular}




\begin{tabular}{|c|c|c|c|c|c|}
\hline 5 & $\mathrm{AO} C(9)$ & 0.0 & 0.0 & 0.0 & 0.0 \\
\hline 6 & AO C(14) & 0.0 & 0.0 & 0.0 & 0.0 \\
\hline 7 & $\mathrm{AO} C(4)$ & 0.0 & 0.0 & 0.0 & 0.0 \\
\hline 8 & AO S(13) & -0.1 & -0.2 & -0.2 & -0.2 \\
\hline 9 & AO S(8) & -0.1 & -0.3 & -0.6 & -0.3 \\
\hline 10 & AO S(3) & -0.2 & 0.3 & 0.1 & 0.1 \\
\hline 11 & AO S(13) & -0.5 & -0.4 & 0.1 & -0.2 \\
\hline 12 & AO S(8) & -0.2 & 0.2 & 0.1 & 0.1 \\
\hline 13 & AO S(3) & 0.2 & -0.1 & 0.1 & 0.1 \\
\hline 14 & AO S(13) & 0.3 & 0.1 & 0.3 & 0.2 \\
\hline 15 & $\mathrm{AO} S(8)$ & 0.2 & -0.2 & 0.1 & 0.0 \\
\hline 16 & AO S(3) & 0.3 & 0.2 & 0.2 & 0.2 \\
\hline 17 & AO S(13) & 0.2 & 0.3 & 0.1 & 0.2 \\
\hline 18 & AO S(8) & 0.3 & 0.3 & 0.2 & 0.3 \\
\hline 19 & AO S(3) & -0.2 & -0.3 & -0.6 & -0.4 \\
\hline 20 & AO Si(2) & 66.0 & 77.9 & 81.4 & 75.1 \\
\hline 21 & AO Si(2) & 81.4 & 78.2 & 73.6 & 77.7 \\
\hline 22 & AO Si(2) & 76.1 & 70.3 & 76.4 & 74.3 \\
\hline 23 & AO Si(2) & 77.9 & 78.5 & 69.2 & 75.2 \\
\hline 24 & AO Li(1) & 0.0 & 0.0 & 0.0 & 0.0 \\
\hline 25 & BOND H(11)-C(9) & 0.2 & -1.3 & -0.2 & -0.4 \\
\hline 26 & BOND H(17)-C(14) & -1.9 & 1.1 & 0.0 & -0.3 \\
\hline 27 & BOND H(6)-C(4) & -0.2 & 0.5 & -0.1 & 0.1 \\
\hline 28 & BOND S(13)-Si(2) & -167.5 & -160.2 & -37.9 & -121.9 \\
\hline 29 & LP S(8) & 4.2 & -13.0 & -8.8 & -5.9 \\
\hline 30 & LP S(3) & -2.6 & 6.8 & -0.8 & 1.1 \\
\hline 31 & BOND H(16)-C(14) & -2.1 & 0.5 & 0.0 & -0.5 \\
\hline 32 & BOND H(15)-C(14) & -0.2 & -0.2 & 0.2 & -0.1 \\
\hline 33 & BOND H(7)-C(4) & -0.6 & -0.1 & -0.6 & -0.4 \\
\hline 34 & BOND H(10)-C(9) & -0.4 & -1.8 & 1.0 & -0.4 \\
\hline 35 & BOND H(12)-C(9) & 0.6 & -0.2 & -0.6 & -0.1 \\
\hline 36 & BOND H(5)-C(4) & -2.1 & -0.6 & -1.5 & -1.4 \\
\hline 37 & BOND C(4)-S(3) & -5.7 & 1.9 & -2.3 & -2.0 \\
\hline 38 & BOND S(3)-Si(2) & -137.7 & -71.8 & -149.7 & -119.7 \\
\hline 39 & BOND S(8)-Si(2) & -61.0 & -149.7 & -134.7 & -115.1 \\
\hline 40 & BOND C(14)-S(13) & -1.6 & -0.9 & 2.3 & -0.1 \\
\hline 41 & LP S(8) & 8.8 & -1.4 & -6.0 & 0.5 \\
\hline 42 & LP S(13) & -11.7 & -5.7 & 0.3 & -5.7 \\
\hline 43 & LP S(3) & 1.2 & -0.3 & -6.6 & -1.9 \\
\hline 44 & BOND C(9)-S(8) & 2.5 & -1.2 & -2.1 & -0.3 \\
\hline 45 & LP S(13) & -5.1 & -7.0 & 7.4 & -1.6 \\
\hline 46 & BOND Si(2)-Li(1) & -177.4 & -122.6 & -124.1 & -141.4 \\
\hline
\end{tabular}

Table 170. NBO Analysis for bonds to silicon in $\mathrm{Me}_{3} \mathrm{SiCl}$ [BP86/BIII//B3LYP/6-31+G(d)].

\begin{tabular}{ccccccc} 
bond & atom & $\begin{array}{c}\text { polarization } \\
\text { of NBO }\end{array}$ & $\mathrm{s}(\%)$ & $\begin{array}{c}\text { Hybridization in } \\
\mathrm{p}(\%)\end{array}$ & $\mathrm{d}(\%)$ & $\begin{array}{c}\text { overall } \\
\text { Occupancy }\end{array}$ \\
\hline $\mathrm{Si}-\mathrm{Cl}$ & $\mathrm{Si}$ & $22.47 \%$ & $0.47(17.85 \%)$ & $4.54(81.01 \%)$ & $0.06(1.14 \%)$ & 1.97375 \\
$\mathrm{Si}-\mathrm{C}$ & $\mathrm{Cl}$ & $77.53 \%$ & $0.88(26.14 \%)$ & $2.79(73.03 \%)$ & $0.03(0.83 \%)$ & \\
$(3 \mathrm{x})$ & $\mathrm{C}$ & $26.81 \%$ & $0.52(27.41 \%)$ & $2.63(71.97 \%)$ & $0.02(0.63 \%)$ & 1.96908 \\
\hline
\end{tabular}

Table 171. NBO Analysis for bonds to silicon in $\mathrm{Me}_{3} \mathrm{SiLi}$ [BP86/BIII//B3LYP/6-31+G(d)].

\begin{tabular}{ccccccc} 
bond & atom & $\begin{array}{c}\text { polarization } \\
\text { of NBO }\end{array}$ & $\mathrm{S}(\%)$ & $\begin{array}{c}\text { Hybridization in } \\
\mathrm{p}(\%)\end{array}$ & $\mathrm{d}(\%)$ & $\begin{array}{c}\text { overall } \\
\text { Occupancy }\end{array}$ \\
\hline $\mathrm{Si}-\mathrm{Li}$ & $\mathrm{Si}$ & $80.37 \%$ & $0.8965(19.08 \%)$ & $4.21(80.40 \%)$ & $0.03(0.52 \%)$ & 1.86023 \\
$\mathrm{Si}-\mathrm{C}$ & $\mathrm{Li}$ & $19.63 \%$ & $0.4431(97.68 \%)$ & $0.02(2.32 \%)$ & & 1.97510
\end{tabular}


Table 172. NBO Analysis for bonds to silicon in (MeO)Me ${ }_{2} \mathrm{SiCl}$ [BP86/BIII//B3LYP/6-31+G(d)].

\begin{tabular}{ccccccc} 
bond & atom & $\begin{array}{c}\text { polarization } \\
\text { of NBO }\end{array}$ & $\mathrm{s}(\%)$ & $\begin{array}{c}\text { Hybridization in } \\
\mathrm{p}(\%)\end{array}$ & $\mathrm{d}(\%)$ & $\begin{array}{c}\text { overall } \\
\text { Occupancy }\end{array}$ \\
\hline \multirow{2}{*}{$\mathrm{Si}-\mathrm{C}$} & $\mathrm{Si}$ & $(25.89 \%)$ & $0.51(28.61 \%)$ & $2.47(70.62 \%)$ & $0.03(0.77 \%)$ & $(1.96519)$ \\
& $\mathrm{C}$ & $(74.11 \%)$ & $0.86(30.81 \%)$ & $2.24(68.92 \%)$ & $0.01(0.27 \%)$ & \\
$\mathrm{Si}-\mathrm{Cl}$ & $\mathrm{Si}$ & $(21.62 \%)$ & $0.47(18.37 \%)$ & $4.36(80.13 \%)$ & $0.08(1.50 \%)$ & $(1.97024)$ \\
& $\mathrm{C}$ & $(78.38 \%)$ & $0.89(25.37 \%)$ & $2.91(73.80 \%)$ & $0.03(0.83 \%)$ & \\
$\mathrm{Si}-\mathrm{O}$ & $\mathrm{Si}$ & $(13.99 \%)$ & $0.37(23.81 \%)$ & $3.14(74.66 \%)$ & $0.06(1.54 \%)$ & $(1.97762)$ \\
& $\mathrm{O}$ & $(86.01 \%)$ & $0.93(39.33 \%)$ & $1.53(60.34 \%)$ & $0.01(0.33 \%)$ & \\
$\mathrm{Si}-\mathrm{C}$ & $\mathrm{Si}$ & $(26.28 \%)$ & $0.51(29.26 \%)$ & $2.39(70.03 \%)$ & $0.02(0.71 \%)$ & $(1.96546)$ \\
& $\mathrm{C}$ & $(73.72 \%)$ & $0.86(30.91 \%)$ & $2.23(68.81 \%)$ & $0.01(0.29 \%)$ & \\
\hline
\end{tabular}

Table 173. NBO Analysis for bonds to silicon in (MeO)Me ${ }_{2} \mathrm{SiLi}$ [BP86/BIII//B3LYP/6-31+G(d)].

\begin{tabular}{ccccccc} 
bond & atom & $\begin{array}{c}\text { polarization } \\
\text { of NBO }\end{array}$ & $\mathrm{s}(\%)$ & $\begin{array}{c}\text { Hybridization in } \\
\mathrm{p}(\%)\end{array}$ & $\mathrm{d}(\%)$ & overall \\
& Occupancy \\
\hline \multirow{2}{*}{$\mathrm{Si}-\mathrm{C}$} & $\mathrm{Si}$ & $26.42 \%$ & $0.51(29.06 \%)$ & $2.42(70.26 \%)$ & $0.02(0.68 \%)$ & 1.97232 \\
& $\mathrm{C}$ & $73.58 \%$ & $0.86(30.97 \%)$ & $2.22(68.81 \%)$ & $0.01(0.23 \%)$ & \\
$\mathrm{Si}-\mathrm{Li}$ & $\mathrm{Si}$ & $79.70 \%$ & $0.89(20.48 \%)$ & $3.85(78.84 \%)$ & $0.03(0.67 \%)$ & 1.83820 \\
& $\mathrm{Li}$ & $20.30 \%$ & $0.45(97.47 \%)$ & $0.03(2.53 \%)$ & - & \\
$\mathrm{Si}-\mathrm{O}$ & $\mathrm{Si}$ & $14.23 \%$ & $0.38(23.50 \%)$ & $3.21(75.36 \%)$ & $0.05(1.15 \%)$ & 1.97730 \\
& $\mathrm{O}$ & $85.77 \%$ & $0.93(35.28 \%)$ & $1.82(64.32 \%)$ & $0.01(0.40 \%)$ & \\
$\mathrm{Si}-\mathrm{C}$ & $\mathrm{Si}$ & $26.52 \%$ & $0.52(28.70 \%)$ & $2.46(70.66 \%)$ & $0.02(0.63 \%)$ & 1.97138 \\
\hline
\end{tabular}

Table 174. NBO Analysis for bonds to silicon in $\left(\mathrm{Me}_{2} \mathrm{~N}\right) \mathrm{Me}_{2} \mathrm{SiCl}$ [BP86/BIII//B3LYP/6-31+G(d)].

\begin{tabular}{ccccccc} 
bond & atom & $\begin{array}{c}\text { polarization } \\
\text { of NBO }\end{array}$ & $\mathrm{s}(\%)$ & $\begin{array}{c}\text { Hybridization in } \\
\mathrm{p}(\%)\end{array}$ & $\mathrm{d}(\%)$ & $\begin{array}{c}\text { overall } \\
\text { Occupancy }\end{array}$ \\
\hline $\mathrm{Cl}-\mathrm{Si}$ & $\mathrm{Si}$ & $21.68 \%$ & $0.47(16.97 \%)$ & $4.81(81.66 \%)$ & $0.08(1.37 \%)$ & 1.96744 \\
& $\mathrm{Cl}$ & $78.32 \%$ & $0.89(25.96 \%)$ & $2.82(73.25 \%)$ & $0.03(0.80 \%)$ & \\
$\mathrm{Si}-\mathrm{N}$ & $\mathrm{Si}$ & $17.07 \%$ & $0.41(26.24 \%)$ & $2.76(72.53 \%)$ & $0.05(1.24 \%)$ & 1 \\
& $\mathrm{~N}$ & $82.93 \%$ & $0.91(34.90 \%)$ & $1.86(64.86 \%)$ & $0.01(0.24 \%)$ & \\
& $\mathrm{Si}$ & $26.56 \%$ & $0.52(28.43 \%)$ & $2.49(70.90 \%)$ & $0.02(0.67 \%)$ & 1.96607 \\
$\mathrm{Si}-\mathrm{C}$ & $\mathrm{C}$ & $73.44 \%$ & $0.86(31.76 \%)$ & $2.14(67.96 \%)$ & $0.01(0.27 \%)$ & \\
& $\mathrm{Si}$ & $26.56 \%$ & $0.52(28.42 \%)$ & $2.49(70.91 \%)$ & $0.02(0.67 \%)$ & 1.96609 \\
$\mathrm{Si}-\mathrm{C}$ & $\mathrm{C}$ & $73.44 \%$ & $0.86(31.76 \%)$ & $2.14(67.96 \%)$ & $0.01(0.27 \%)$ & 1.96609 \\
\hline
\end{tabular}

Table 175. NBO Analysis for bonds to silicon in $\left(\mathrm{Me}_{2} \mathrm{~N}\right) \mathrm{Me}_{2} \mathrm{SiLi}$ [BP86/BIII//B3LYP/6-31+G(d)].

$\begin{array}{ccccccc}\text { bond } & \text { atom } & \begin{array}{c}\text { polarization } \\ \text { of NBO }\end{array} & \mathrm{s}(\%) & \begin{array}{c}\text { Hybridization in } \\ \mathrm{p}(\%)\end{array} & \mathrm{d}(\%) & \begin{array}{c}\text { overall } \\ \text { Occupancy }\end{array} \\ & \mathrm{Si} & 78.19 \% & 0.88(17.51 \%) & 4.67(81.84 \%) & 0.04(0.64 \%) & 1.82171 \\ \mathrm{Li}-\mathrm{Si} & \mathrm{Li} & 21.81 \% & 0.47(98.21 \%) & 0.02(1.79 \%) & & \\ & \mathrm{Si} & 18.36 \% & 0.43(26.46 \%) & 2.75(72.67 \%) & 0.03(0.87 \%) & 1.95402 \\ \mathrm{Si}-\mathrm{N} & \mathrm{N} & 81.64 \% & 0.90(27.19 \%) & 2.67(72.50 \%) & 0.01(0.31 \%) & \\ & \mathrm{Si} & 27.29 \% & 0.52(28.69 \%) & 2.47(70.79 \%) & 0.02(0.52 \%) & 1 \\ \mathrm{Si}-\mathrm{C} & \mathrm{C} & 72.71 \% & 0.85(31.55 \%) & 2.16(68.21 \%) & 0.01(0.24 \%) & \\ & \mathrm{Si} & 27.29 \% & 0.52(28.69 \%) & 2.47(70.79 \%) & 0.02(0.52 \%) & 1 \\ \mathrm{Si}-\mathrm{C} & \mathrm{C} & 72.71 \% & 0.85(31.55 \%) & 2.16(68.21 \%) & 0.01(0.24 \%) & \\ \end{array}$

Table 176. NBO Analysis for bonds to silicon in (MeS) $\mathrm{Me}_{2} \mathrm{SiCl}$ [BP86/BIII//B3LYP/6-31+G(d)]. 


\begin{tabular}{ccccccc} 
bond & atom & $\begin{array}{c}\text { polarization } \\
\text { of NBO }\end{array}$ & $\mathrm{s}(\%)$ & $\begin{array}{c}\text { Hybridization in } \\
\text { p (\%) }\end{array}$ & $\mathrm{d}(\%)$ & $\begin{array}{c}\text { overall } \\
\text { Occupancy }\end{array}$ \\
\hline \multirow{2}{*}{$\mathrm{Si}-\mathrm{C}$} & $\mathrm{Si}$ & $27.67 \%$ & $0.5260(29.41 \%)$ & $2.38(69.99 \%)$ & $0.02(0.60 \%)$ & \multirow{2}{*}{.96835} \\
& $\mathrm{C}$ & $72.33 \%$ & $0.8505(31.21 \%)$ & $2.20(68.54 \%)$ & $0.01(0.24 \%)$ & \\
$\mathrm{Si}-\mathrm{Cl}$ & $\mathrm{Si}$ & $23.19 \%$ & $0.4815(19.09 \%)$ & $4.17(79.64 \%)$ & $0.07(1.26 \%)$ & 1.97471 \\
& $\mathrm{Cl}$ & $76.81 \%$ & $0.8764(25.98 \%)$ & $2.82(73.20 \%)$ & $0.03(0.82 \%)$ & \\
$\mathrm{Si}-\mathrm{S}$ & $\mathrm{Si}$ & $30.21 \%$ & $0.5497(22.72 \%)$ & $3.36(76.29 \%)$ & $0.04(1.00 \%)$ & 1 \\
& $\mathrm{~S}$ & $69.79 \%$ & $0.8354(20.76 \%)$ & $3.76(78.04 \%)$ & $0.06(1.20 \%)$ & 1.95872 \\
$\mathrm{Si}-\mathrm{C}$ & $\mathrm{Si}$ & $27.71 \%$ & $0.5264(28.83 \%)$ & $2.45(70.59 \%)$ & $0.02(0.58 \%)$ & 1 \\
& $\mathrm{C}$ & $72.29 \%$ & $0.8502(31.50 \%)$ & $2.17(68.24 \%)$ & $0.01(0.25 \%)$ & 1.96839 \\
\hline
\end{tabular}

Table 177. NBO Analysis for bonds to silicon in (MeS)Me ${ }_{2}$ SiLi [BP86/BIII//B3LYP/6-31+G(d)].

$\begin{array}{ccccccc}\text { bond } & \text { atom } & \begin{array}{c}\text { polarization } \\ \text { of NBO }\end{array} & \mathrm{s}(\%) & \begin{array}{c}\text { Hybridization in } \\ \mathrm{p}(\%)\end{array} & \mathrm{d}(\%) & \text { overall } \\ \text { Occupancy }\end{array}$

Table 178. NBO Analysis for bonds to silicon in (MeO) ${ }_{2} \mathrm{MeSiCl}$ [BP86/BIII//B3LYP/6-31+G(d)].

\begin{tabular}{|c|c|c|c|c|c|c|}
\hline bond & atom & polarization & s (\%) & Hybridization in & $d(\%)$ & $\begin{array}{c}\text { overall } \\
\text { Occupancy }\end{array}$ \\
\hline \multirow{2}{*}{$\mathrm{Si}-\mathrm{C}$} & Si & $25.89 \%$ & $0.51(28.61 \%)$ & $2.47(70.62 \%)$ & $0.03(0.77 \%)$ & \multirow[b]{2}{*}{1.96519} \\
\hline & C & $74.11 \%$ & $0.86(30.81 \%)$ & $2.24(68.92 \%)$ & $0.01(0.27 \%)$ & \\
\hline \multirow[b]{2}{*}{$\mathrm{Si}-\mathrm{Cl}$} & $\mathrm{Si}$ & $21.62 \%$ & $0.47(18.37 \%)$ & $4.36(80.13 \%)$ & $0.08(1.50 \%)$ & \multirow{2}{*}{1.97024} \\
\hline & $\mathrm{Cl}$ & $78.38 \%$ & $0.89(25.37 \%)$ & $2.91(73.80 \%)$ & $0.03(0.83 \%)$ & \\
\hline \multirow{2}{*}{ Si-O } & Si & $13.99 \%$ & $0.37(23.81 \%)$ & $3.14(74.66 \%)$ & $0.06(1.54 \%)$ & \multirow{2}{*}{1.97762} \\
\hline & 0 & $86.01 \%$ & $0.93(39.33 \%)$ & $1.53(60.34 \%)$ & $0.01(0.33 \%)$ & \\
\hline \multirow{2}{*}{$\mathrm{Si}-\mathrm{C}$} & $\mathrm{Si}$ & $26.28 \%$ & $0.51(29.26 \%)$ & $2.39(70.03 \%)$ & $0.02(0.71 \%)$ & \multirow{2}{*}{1.96546} \\
\hline & $\mathrm{O}$ & $73.72 \%$ & $0.86(30.91 \%)$ & $2.23(68.81 \%)$ & $0.01(0.29 \%)$ & \\
\hline
\end{tabular}

Table 179. NBO Analysis for bonds to silicon in (MeO) ${ }_{2} \mathrm{MeSiLi}$ [BP86/BIII//B3LYP/6-31+G(d)].

\begin{tabular}{ccccccc} 
bond & atom & $\begin{array}{c}\text { polarization } \\
\text { of NBO }\end{array}$ & $\mathrm{s}(\%)$ & $\begin{array}{c}\text { Hybridization in } \\
\mathrm{p}(\%)\end{array}$ & $\mathrm{d}(\%)$ & $\begin{array}{c}\text { overall } \\
\text { Occupancy }\end{array}$ \\
\hline \multirow{2}{*}{$\mathrm{Li}-\mathrm{Si}$} & $\mathrm{Si}$ & $83.01 \%$ & $0.91(23.71 \%)$ & $3.19(75.56 \%)$ & $0.03(0.73 \%)$ & \multirow{2}{*}{1.82773} \\
& $\mathrm{Li}$ & $16.99 \%$ & $0.41(96.43 \%)$ & $0.04(3.57 \%)$ & - & \\
\multirow{2}{*}{$\mathrm{Si}-\mathrm{O}$} & $\mathrm{Si}$ & $13.54 \%$ & $0.37(24.40 \%)$ & $3.04(74.24 \%)$ & $0.06(1.35 \%)$ & 1.97614 \\
& $\mathrm{O}$ & $86.46 \%$ & $0.93(35.48 \%)$ & $1.81(64.11 \%)$ & $0.01(0.41 \%)$ & \\
$\mathrm{Si}-\mathrm{O}$ & $\mathrm{Si}$ & $13.25 \%$ & $0.36(23.50 \%)$ & $3.19(75.08 \%)$ & $0.06(1.42 \%)$ & 1.97713 \\
& $\mathrm{O}$ & $86.75 \%$ & $0.93(37.42 \%)$ & $1.66(62.21 \%)$ & $0.01(0.37 \%)$ & \\
$\mathrm{Si}-\mathrm{C}$ & $\mathrm{Si}$ & $25.33 \%$ & $0.50(29.75 \%)$ & $2.33(69.39 \%)$ & $0.03(0.86 \%)$ & \multirow{2}{*}{1.96598} \\
\hline
\end{tabular}

Table 180. NBO Analysis for bonds to silicon in $\left(\mathrm{Me}_{2} \mathrm{~N}\right)_{2} \mathrm{MeSiCl}$ [BP86/BIII//B3LYP/6-31+G(d)].

\begin{tabular}{ccccccc} 
bond & atom & $\begin{array}{c}\text { polarization } \\
\text { of NBO }\end{array}$ & $\mathrm{s}(\%)$ & $\begin{array}{c}\text { Hybridization in } \\
\mathrm{p} \mathrm{( \% )}\end{array}$ & $\mathrm{d}(\%)$ & $\begin{array}{c}\text { overall } \\
\text { Occupancy }\end{array}$ \\
\hline $\mathrm{Si}-\mathrm{Cl}$ & $\mathrm{Si}$ & $21.69 \%$ & $0.47(17.83 \%)$ & $4.53(80.76 \%)$ & $0.08(1.42 \%)$ & 1.96608
\end{tabular}




\begin{tabular}{lllllll} 
& $\mathrm{Si}$ & $25.92 \%$ & $0.51(28.82 \%)$ & $2.44(70.39 \%)$ & $0.03(0.79 \%)$ & 1 \\
Si-C & $\mathrm{C}$ & $74.08 \%$ & $0.86(31.69 \%)$ & $2.15(68.03 \%)$ & $0.01(0.28 \%)$ & \\
& $\mathrm{Si}$ & $16.69 \%$ & $0.41(26.71 \%)$ & $2.70(72.01 \%)$ & $0.05(1.29 \%)$ & 1 \\
$\mathrm{Si}-\mathrm{N}$ & $\mathrm{N}$ & $83.31 \%$ & $0.91(37.07 \%)$ & $1.69(62.71 \%)$ & $0.01(0.22 \%)$ & \\
& $\mathrm{Si}$ & $16.99 \%$ & $0.41(26.68 \%)$ & $2.70(72.09 \%)$ & $0.05(1.23 \%)$ & 1 \\
$\mathrm{Si}-\mathrm{N}$ & $\mathrm{N}$ & $83.01 \%$ & $0.91(38.17 \%)$ & $1.61(61.62 \%)$ & $0.01(0.21 \%)$ & \\
\hline
\end{tabular}

Table 181. NBO Analysis for bonds to silicon in $\left(\mathrm{Me}_{2} \mathrm{~N}\right)_{2} \mathrm{MeSiLi}$ [BP86/BIII//B3LYP/6-31+G(d)].

\begin{tabular}{ccccccc} 
bond & atom & $\begin{array}{c}\text { polarization } \\
\text { of NBO }\end{array}$ & $\mathrm{s}(\%)$ & $\begin{array}{c}\text { Hybridization in } \\
\mathrm{p}(\%)\end{array}$ & $\mathrm{d}(\%)$ & $\begin{array}{c}\text { overall } \\
\text { Occupancy }\end{array}$ \\
\hline $\mathrm{Si}-\mathrm{Li}$ & $\mathrm{Si}$ & $80.49 \%$ & $0.90(18.42 \%)$ & $4.40(80.99 \%)$ & $0.03(0.59 \%)$ & 1.81797 \\
& $\mathrm{Li}$ & $19.51 \%$ & $0.44(97.99 \%)$ & $0.02(2.01 \%)$ & - & \\
$\mathrm{Si}-\mathrm{N}$ & $\mathrm{Si}$ & $17.61 \%$ & $0.42(26.14 \%)$ & $2.79(72.88 \%)$ & $0.04(0.98 \%)$ & 1.95522 \\
& $\mathrm{~N}$ & $82.39 \%$ & $0.91(29.00 \%)$ & $2.44(70.71 \%)$ & $0.01(0.29 \%)$ & \\
$\mathrm{Si}-\mathrm{N}$ & $\mathrm{Si}$ & $17.28 \%$ & $0.42(27.05 \%)$ & $2.66(72.04 \%)$ & $0.03(0.91 \%)$ & 1.96932 \\
& $\mathrm{~N}$ & $82.72 \%$ & $0.91(37.40 \%)$ & $1.67(62.38 \%)$ & $0.01(0.22 \%)$ & \\
$\mathrm{Si}-\mathrm{C}$ & $\mathrm{Si}$ & $26.40 \%$ & $0.51(29.18 \%)$ & $2.41(70.18 \%)$ & $0.02(0.65 \%)$ & 1.96693 \\
\hline
\end{tabular}

Table 182. NBO Analysis for bonds to silicon in (MeS) ${ }_{2} \mathrm{MeSiCl}$ [BP86/BIII//B3LYP/6-31+G(d)].

\begin{tabular}{ccccccc} 
bond & atom & $\begin{array}{c}\text { polarization } \\
\text { of NBO }\end{array}$ & $\mathrm{s}(\%)$ & $\begin{array}{c}\text { Hybridization in } \\
\mathrm{p}(\%)\end{array}$ & $\mathrm{d}(\%)$ & $\begin{array}{c}\text { overall } \\
\text { Occupancy }\end{array}$ \\
\hline $\mathrm{Si}-\mathrm{Cl}$ & $\mathrm{Si}$ & $24.17 \%$ & $0.49(20.38 \%)$ & $3.84(78.31 \%)$ & $0.06(1.31 \%)$ & 1.97609 \\
& $\mathrm{Cl}$ & $75.83 \%$ & $0.87(26.30 \%)$ & $2.77(72.86 \%)$ & $0.03(0.85 \%)$ & \\
$\mathrm{Si}-\mathrm{S}$ & $\mathrm{Si}$ & $31.40 \%$ & $0.56(24.29 \%)$ & $3.07(74.63 \%)$ & $0.04(1.08 \%)$ & 1.95803 \\
& $\mathrm{~S}$ & $68.60 \%$ & $0.83(20.06 \%)$ & $3.93(78.76 \%)$ & $0.06(1.18 \%)$ & \\
$\mathrm{Si}-\mathrm{S}$ & $\mathrm{Si}$ & $31.06 \%$ & $0.56(24.28 \%)$ & $3.07(74.64 \%)$ & $0.04(1.08 \%)$ & 1.95967 \\
& $\mathrm{~S}$ & $68.94 \%$ & $0.83(20.49 \%)$ & $3.82(78.34 \%)$ & $0.06(1.17 \%)$ & \\
$\mathrm{Si}-\mathrm{C}$ & $\mathrm{Si}$ & $28.50 \%$ & $0.53(31.07 \%)$ & $2.20(68.38 \%)$ & $0.02(0.54 \%)$ & 1 \\
& $\mathrm{C}$ & $71.50 \%$ & $0.85(31.04 \%)$ & $2.21(68.72 \%)$ & $0.01(0.24 \%)$ & 1.96865 \\
\hline
\end{tabular}

Table 183. NBO Analysis for bonds to silicon in (MeS) ${ }_{2}$ MeSiLi [BP86/BIII//B3LYP/6-31+G(d)].

\begin{tabular}{ccccccc} 
bond & atom & $\begin{array}{c}\text { polarization } \\
\text { of NBO }\end{array}$ & $\mathrm{s}(\%)$ & $\begin{array}{c}\text { Hybridization in } \\
\text { p (\%) }\end{array}$ & $\mathrm{d}(\%)$ & $\begin{array}{c}\text { overall } \\
\text { Occupancy }\end{array}$ \\
\hline \multirow{2}{*}{$\mathrm{Si}-\mathrm{Li}$} & $\mathrm{Si}$ & $89.08 \%$ & $0.94(22.31 \%)$ & $3.46(77.22 \%)$ & $0.02(0.47 \%)$ & 1.84130 \\
& $\mathrm{Li}$ & $10.92 \%$ & $0.33(95.92 \%)$ & $0.04(4.08 \%)$ & - & \\
$\mathrm{Si}-\mathrm{S}$ & $\mathrm{Si}$ & $29.78 \%$ & $0.55(23.03 \%)$ & $3.30(76.10 \%)$ & $0.04(0.87 \%)$ & 1.96552 \\
& $\mathrm{~S}$ & $70.22 \%$ & $0.84(20.09 \%)$ & $3.93(78.89 \%)$ & $0.05(1.02 \%)$ & \\
$\mathrm{Si}-\mathrm{S}$ & $\mathrm{Si}$ & $29.52 \%$ & $0.54(23.88 \%)$ & $3.15(75.27 \%)$ & $0.04(0.85 \%)$ & 1.96676 \\
& $\mathrm{~S}$ & $70.48 \%$ & $0.84(20.90 \%)$ & $3.74(78.22 \%)$ & $0.04(0.89 \%)$ & \\
$\mathrm{Si}-\mathrm{C}$ & $\mathrm{Si}$ & $29.07 \%$ & $0.54(31.59 \%)$ & $2.15(67.84 \%)$ & $0.02(0.57 \%)$ & 1.97313 \\
\hline
\end{tabular}

Table 184. NBO Analysis for bonds to silicon in (MeO) ${ }_{3} \mathrm{MeSiCl}$ [BP86/BIII//B3LYP/6-31+G(d)].

\begin{tabular}{ccccccc} 
bond & atom & $\begin{array}{c}\text { polarization } \\
\text { of NBO }\end{array}$ & $\mathrm{s}(\%)$ & $\begin{array}{c}\text { Hybridization in } \\
\mathrm{p}(\%)\end{array}$ & $\mathrm{d}(\%)$ & $\begin{array}{c}\text { overall } \\
\text { Occupancy }\end{array}$ \\
\hline $\mathrm{Si}-\mathrm{Cl}$ & $\mathrm{Si}$ & $21.56 \%$ & $0.46(21.37 \%)$ & $3.57(76.40 \%)$ & $0.10(2.22 \%)$ & 1.96414 \\
& $\mathrm{Cl}$ & $78.44 \%$ & $0.89(23.05 \%)$ & $3.30(76.04 \%)$ & $0.04(0.91 \%)$ & \\
$\mathrm{Si}-\mathrm{O}$ & $\mathrm{Si}$ & $13.69 \%$ & $0.37(25.65 \%)$ & $2.82(72.42 \%)$ & $0.08(1.93 \%)$ & 1.97434 \\
& $\mathrm{O}$ & $86.31 \%$ & $0.93(36.32 \%)$ & $1.74(63.32 \%)$ & $0.01(0.37 \%)$ & \\
$\mathrm{Si}-\mathrm{O}$ & $\mathrm{Si}$ & $13.96 \%$ & $0.37(26.71 \%)$ & $2.68(71.58 \%)$ & $0.06(1.71 \%)$ & 1.97627 \\
$\mathrm{Si}-\mathrm{O}$ & $\mathrm{Si}$ & $86.04 \%$ & $0.93(37.36 \%)$ & $1.67(62.24 \%)$ & $0.01(0.40 \%)$ & \\
& & $13.87 \%$ & $0.37(26.24 \%)$ & $2.74(72.00 \%)$ & $0.07(1.76 \%)$ & 1.97580
\end{tabular}


Table 185. NBO Analysis for bonds to silicon in (MeO) ${ }_{3} \mathrm{MeSiLi}$ [BP86/BIII//B3LYP/6-31+G(d)].

\begin{tabular}{ccccccc} 
bond & atom & $\begin{array}{c}\text { polarization } \\
\text { of NBO }\end{array}$ & $\mathrm{s}(\%)$ & $\begin{array}{c}\text { Hybridization in } \\
\mathrm{p}(\%)\end{array}$ & $\mathrm{d}(\%)$ & $\begin{array}{c}\text { overall } \\
\text { Occupancy }\end{array}$ \\
\hline $\mathrm{Li}-\mathrm{Si}$ & $\mathrm{Si}$ & $83.67 \%$ & $0.91(26.35 \%)$ & $2.76(72.66 \%)$ & $0.04(0.99 \%)$ & 1.80536 \\
& $\mathrm{Li}$ & $16.33 \%$ & $0.40(95.70 \%)$ & $0.04(4.30 \%)$ & - & \\
$\mathrm{Si}-\mathrm{O}$ & $\mathrm{Si}$ & $12.96 \%$ & $0.36(25.10 \%)$ & $2.92(73.27 \%)$ & $0.06(1.63 \%)$ & 1.97719 \\
& $\mathrm{O}$ & $87.04 \%$ & $0.93(37.52 \%)$ & $1.65(62.07 \%)$ & $0.01(0.42 \%)$ & \\
$\mathrm{Si}-\mathrm{O}$ & $\mathrm{Si}$ & $13.28 \%$ & $0.36(24.60 \%)$ & $3.00(73.84 \%)$ & $0.06(1.56 \%)$ & 1.97557 \\
& $\mathrm{O}$ & $86.72 \%$ & $0.93(35.37 \%)$ & $1.81(64.17 \%)$ & $0.01(0.46 \%)$ & \\
$\mathrm{Si}-\mathrm{O}$ & $\mathrm{Si}$ & $13.10 \%$ & $0.36(25.76 \%)$ & $2.82(72.64 \%)$ & $0.06(1.60 \%)$ & 1.97508 \\
& $\mathrm{O}$ & $86.90 \%$ & $0.93(34.62 \%)$ & $1.88(64.94 \%)$ & $0.01(0.44 \%)$ & 1.970 \\
\hline
\end{tabular}

Table 186. NBO Analysis for bonds to silicon in $\left(\mathrm{Me}_{2} \mathrm{~N}\right)_{3} \mathrm{MeSiCl}$ [BP86/BIII//B3LYP/6-31+G(d)].

\begin{tabular}{ccccccc} 
bond & atom & $\begin{array}{c}\text { polarization } \\
\text { of NBO }\end{array}$ & $\mathrm{s}(\%)$ & $\begin{array}{c}\text { Hybridization in } \\
\mathrm{p}(\%)\end{array}$ & $\mathrm{d}(\%)$ & Overall \\
& & $\mathrm{Occ}$ (\%pancy \\
\hline $\mathrm{Cl}-\mathrm{Si}$ & $\mathrm{Si}$ & $21.34 \%$ & $0.46(18.25 \%)$ & $4.43(80.81 \%)$ & $0.05(0.94 \%)$ & 1.95993 \\
& $\mathrm{Cl}$ & $78.66 \%$ & $0.89(26.69 \%)$ & $2.71(72.35 \%)$ & $0.04(0.96 \%)$ & \\
$\mathrm{Si}-\mathrm{N}$ & $\mathrm{Si}$ & $16.43 \%$ & $0.41(27.50 \%)$ & $2.60(71.37 \%)$ & $0.04(1.13 \%)$ & 1.96623 \\
& $\mathrm{~N}$ & $83.57 \%$ & $0.91(39.59 \%)$ & $1.52(60.10 \%)$ & $0.01(0.31 \%)$ & \\
$\mathrm{Si}-\mathrm{N}$ & $\mathrm{Si}$ & $16.36 \%$ & $0.40(26.99 \%)$ & $2.66(71.81 \%)$ & $0.04(1.20 \%)$ & 1.96277 \\
& $\mathrm{~N}$ & $83.64 \%$ & $0.91(37.66 \%)$ & $1.65(62.01 \%)$ & $0.01(0.33 \%)$ & \\
$\mathrm{Si}-\mathrm{N}$ & $\mathrm{Si}$ & $16.40 \%$ & $0.40(27.24 \%)$ & $2.63(71.60 \%)$ & $0.04(1.16 \%)$ & 1 \\
& $\mathrm{~N}$ & $83.60 \%$ & $0.91(39.56 \%)$ & $1.52(60.14 \%)$ & $0.01(0.31 \%)$ & \\
\hline
\end{tabular}

Table 187. NBO Analysis for bonds to silicon in $\left(\mathrm{Me}_{2} \mathrm{~N}\right)_{3} \mathrm{MeSiLi}$ [BP86/BIII//B3LYP/6-31+G(d)].

\begin{tabular}{|c|c|c|c|c|c|c|}
\hline bond & atom & $\begin{array}{l}\text { polarization } \\
\text { of NBO }\end{array}$ & s (\%) & $\begin{array}{l}\text { Hybridization in } \\
\text { p (\%) }\end{array}$ & $d(\%)$ & $\begin{array}{c}\text { overall } \\
\text { Occupancy }\end{array}$ \\
\hline \multirow{2}{*}{$\mathrm{Si}-\mathrm{Li}$} & $\mathrm{Si}$ & $82.38 \%$ & $0.91(21.59 \%)$ & $3.60(77.68 \%)$ & $0.03(0.73 \%)$ & \multirow{2}{*}{1.82598} \\
\hline & $\mathrm{Li}$ & $17.62 \%$ & $0.42(98.04 \%)$ & $0.02(1.96 \%)$ & 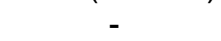 & \\
\hline \multirow{2}{*}{$\mathrm{Si}-\mathrm{N}$} & $\mathrm{Si}$ & $16.33 \%$ & 0.40 (26.31 \%) & $2.76(72.72 \%)$ & 0.04 (0.97 \%) & \multirow{2}{*}{1.96679} \\
\hline & $\mathrm{N}$ & $83.67 \%$ & $0.91(39.05 \%)$ & $1.55(60.68 \%)$ & $0.01(0.27 \%)$ & \\
\hline \multirow{2}{*}{$\mathrm{Si}-\mathrm{N}$} & $\mathrm{Si}$ & $16.35 \%$ & $0.40(26.25 \%)$ & $2.77(72.78 \%)$ & $0.04(0.97 \%)$ & \multirow{2}{*}{1.96667} \\
\hline & $\mathrm{N}$ & $83.65 \%$ & $0.91(39.04 \%)$ & $1.55(60.69 \%)$ & $0.01(0.27 \%)$ & \\
\hline \multirow{2}{*}{$\mathrm{Si}-\mathrm{N}$} & $\mathrm{Si}$ & $16.31 \%$ & $0.40(26.28 \%)$ & $2.77(72.75 \%)$ & $0.04(0.97 \%)$ & \multirow{2}{*}{1.96683} \\
\hline & $\mathrm{N}$ & $83.69 \%$ & $0.91(39.10 \%)$ & $1.55(60.64 \%)$ & $0.01(0.27 \%)$ & \\
\hline
\end{tabular}

Table 188. NBO Analysis for bonds to silicon in (MeS) ${ }_{3} \mathrm{MeSiCl}$ [BP86/BIII//B3LYP/6-31+G(d)].

\begin{tabular}{ccccccc} 
bond & atom & $\begin{array}{c}\text { polarization } \\
\text { of NBO }\end{array}$ & $\mathrm{s}(\%)$ & $\begin{array}{c}\text { Hybridization in } \\
\mathrm{p}(\%)\end{array}$ & $\mathrm{d}(\%)$ & $\begin{array}{c}\text { overall } \\
\text { Occupancy }\end{array}$ \\
\hline \multirow{2}{*}{$\mathrm{Si}-\mathrm{Cl}$} & $\mathrm{Si}$ & $75.20 \%$ & $0.87(25.95 \%)$ & $2.82(73.22 \%)$ & $0.03(0.83 \%)$ & \multirow{2}{*}{1.97693} \\
& $\mathrm{Cl}$ & $24.80 \%$ & $0.50(21.66 \%)$ & $3.56(77.01 \%)$ & $0.06(1.33 \%)$ & \\
\multirow{2}{*}{$\mathrm{Si}-\mathrm{S}$} & $\mathrm{Si}$ & $32.13 \%$ & $0.57(26.07 \%)$ & $2.79(72.79 \%)$ & $0.04(1.14 \%)$ & 1.95960 \\
& $\mathrm{~S}$ & $67.87 \%$ & $0.82(20.02 \%)$ & $3.94(78.83 \%)$ & $0.06(1.15 \%)$ & \\
\multirow{2}{*}{$\mathrm{Si}-\mathrm{S}$} & $\mathrm{Si}$ & $32.69 \%$ & $0.57(26.10 \%)$ & $2.79(72.82 \%)$ & $0.04(1.08 \%)$ & 1 \\
& $\mathrm{~S}$ & $67.31 \%$ & $0.82(20.07 \%)$ & $3.92(78.74 \%)$ & $0.06(1.19 \%)$ & \\
$\mathrm{Si}-\mathrm{S}$ & $\mathrm{Si}$ & $32.48 \%$ & $0.57(26.15 \%)$ & $2.78(72.76 \%)$ & $0.04(1.09 \%)$ & \multirow{2}{*}{1.96134} \\
\hline
\end{tabular}

Table 189. NBO Analysis for bonds to silicon in (MeS) ${ }_{3} \mathrm{MeSiLi}$ [BP86/BIII//B3LYP/6-31+G(d)]. 


\begin{tabular}{|c|c|c|c|c|c|c|}
\hline bond & atom & $\begin{array}{l}\text { polarization } \\
\text { of } \mathrm{NBO}\end{array}$ & s (\%) & ybridization in & $d(\%)$ & $\begin{array}{c}\text { overall } \\
\text { Occunancy }\end{array}$ \\
\hline \multirow[b]{2}{*}{$\mathrm{Si}-\mathrm{Li}$} & $\mathrm{Si}$ & $91.08 \%$ & $0.9544(22.92 \%)$ & $3.34(76.55 \%)$ & $0.02(0.53 \%)$ & \multirow[b]{2}{*}{1.82917} \\
\hline & $\mathrm{Li}$ & $8.92 \%$ & $0.2986(94.77 \%)$ & $0.06(5.23 \%)$ & & \\
\hline \multirow{2}{*}{ Si-S } & $\mathrm{Si}$ & $30.14 \%$ & $0.5490(27.04 \%)$ & $2.66(72.03 \%)$ & 0.03(0.93 \%) & \multirow{2}{*}{1.97050} \\
\hline & $\mathrm{S}$ & $69.86 \%$ & $0.8358(19.35 \%)$ & $4.12(79.76 \%)$ & $0.05(0.88 \%)$ & \\
\hline \multirow{2}{*}{ Si-S } & $\mathrm{Si}$ & $30.52 \%$ & $0.5524(25.45 \%)$ & $2.90(73.70 \%)$ & $0.03(0.85 \%)$ & \multirow{2}{*}{1.97213} \\
\hline & $\mathrm{S}$ & $69.48 \%$ & $0.8336(20.05 \%)$ & $3.94(78.96 \%)$ & $0.05(0.99 \%)$ & \\
\hline \multirow{2}{*}{ Si-S } & $\mathrm{Si}$ & $30.85 \%$ & $0.5554(25.51 \%)$ & $2.88(73.55 \%)$ & $0.04(0.94 \%)$ & \multirow{2}{*}{1.96852} \\
\hline & $\mathrm{S}$ & $69.15 \%$ & $0.8316(19.92 \%)$ & $3.97(79.04 \%)$ & $0.05(1.04 \%)$ & \\
\hline
\end{tabular}

Table 190. Natural Charges on silicon for selected model systems [BP86/BIII//B3LYP/6-31+G(d)]

\begin{tabular}{cccc} 
model & $\mathrm{Y}$ & \multicolumn{2}{c}{ charge on silicon } \\
& & $\mathrm{X}=\mathrm{Cl}$ & X=Li \\
\hline $\mathrm{Me}_{3} \mathrm{SiX}$ & - & 1.716 & 0.729 \\
$(\mathrm{Y}) \mathrm{Me}_{2} \mathrm{SiX}$ & $\mathrm{OMe}$ & 1.924 & 0.983 \\
& $\mathrm{NMe}_{2}$ & 1.839 & 0.912 \\
& $\mathrm{SMe}$ & 1.509 & 0.543 \\
$(\mathrm{Y})_{2} \mathrm{MeSiX}$ & $\mathrm{OMe}$ & 2.096 & 1.137 \\
& $\mathrm{NMe}_{2}$ & 1.978 & 1.032 \\
& $\mathrm{SMe}$ & 1.292 & 0.329 \\
$(\mathrm{Y})_{3} \mathrm{SiX}$ & $\mathrm{OMe}$ & 2.260 & 1.316 \\
& $\mathrm{NMe}_{2}$ & 2.118 & 1.148 \\
& $\mathrm{SMe}$ & 1.060 & 0.133 \\
\hline
\end{tabular}

